\title{
Oscillations of neutrinos and mesons in quantum field theory
}

\author{
Mikael Beuthe円 \\ Institut de Physique Théorique, Université Catholique de Louvain, B-1348 Louvain-la-Neuve, Belgium円
}

\begin{abstract}
This report deals with the quantum field theory of particle oscillations in vacuum. We first review the various controversies regarding quantum-mechanical derivations of the oscillation formula, as well as the different field-theoretical approaches proposed to settle them. We then clear up the contradictions between the existing field-theoretical treatments by a thorough study of the external wave packet model. In particular, we show that the latter includes stationary models as a subcase. In addition, we compute explicitly decoherence terms, which destroy interferences, in order to prove that the coherence length can be increased without bound by more accurate energy measurements. We show that decoherence originates not only in the width and in the separation of wave packets, but also in their spreading through spacetime. In this review, we neither assume the relativistic limit nor the stability of oscillating particles, so that the oscillation formula derived with field-theoretical methods can be applied not only to neutrinos but also to neutral $K$ and $B$ mesons. Finally, we discuss oscillations of correlated particles in the same framework.
\end{abstract}

PACS codes: 14.60.Pq, 14.40.-n, 03.65.Bz, 12.15.Ff

Keywords: oscillation, neutrino, meson, quantum field theory

\section{Contents}

1 Introduction 4

\begin{tabular}{|lll}
\hline 2 & Oscillations in quantum mechanics & $\mathbf{7}$
\end{tabular}

2.1 Mixing in quantum mechanic . . . . . . . . . . . . . . . . . . . . . . . . . . . 8

2.2 Oscillations with plane waves . . . . . . . . . . . . . . . . . . . . . . . . . . . . . . 11

2.3 Time to space conversion of the oscillating phase . . . . . . . . . . . . . . . . . . . . . . . 13

2.3 .1 Standard oscillation phase . . . . . . . . . . . . . . . . . . . . . . 13

2.3 .2 Non-standard oscillation phase . . . . . . . . . . . . . . . . . . . . . . . 16

2.4 Problems with the plane wave treatment . . . . . . . . . . . . . . . . . . . . . . . . . . . 18

$2.5 \quad$ Intermediate wave packets and other improvements . . . . . . . . . . . . . . . . . . . . . . 19

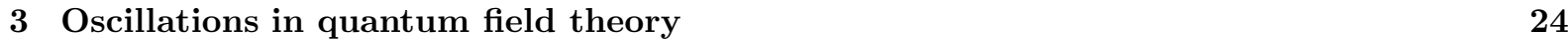

3.1 Review of the literature . . . . . . . . . . . . . . . . . . . . . . . . . . 24

3.2 Five questions . . . . . . . . . . . . . . . . . . . . . . . . . . . . . . 27

4 The external wave packet model 28

4.1 The Jacob-Sachs mode . . . . . . . . . . . . . . . . . . . . . . . . . . 30

4.1 .1 The process . . . . . . . . . . . . . . . . . . . . . . . . . 30

4.1 .2 Wave packets . . . . . . . . . . . . . . . . . . . . . . . . 31

\footnotetext{
${ }^{1}$ Electronic address:mbeuthe@oma.be

${ }^{2}$ Present address: Observatoire Royal de Belgique, Avenue Circulaire 3, B-1180 Bruxelles, Belgium
} 
4.1 .3 The propagation amplitude $\ldots \ldots \ldots \ldots \ldots \ldots \ldots$. . . . . . . . . . . . . 32

4.2 Mixed propagators $\ldots \ldots \ldots \ldots \ldots \ldots \ldots \ldots \ldots \ldots \ldots$

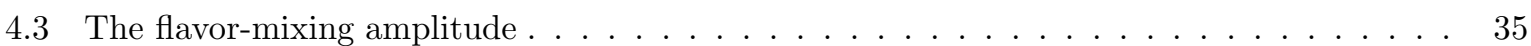

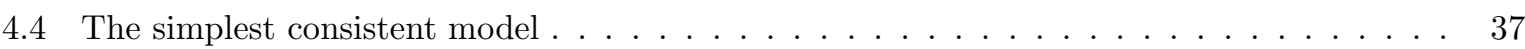

4.5 The overlap function for Gaussian wave packet $\ldots \ldots \ldots$. . . . . . . . . . . 38

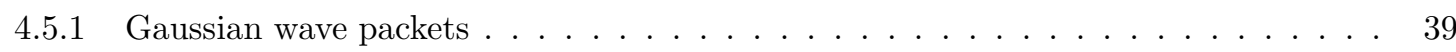

4.5 .2 Gaussian overlap function $\ldots \ldots \ldots \ldots \ldots \ldots \ldots$. . . . . . . . . . . 41

\begin{tabular}{|lll}
5 & Propagation amplitude and dispersion & 43
\end{tabular}

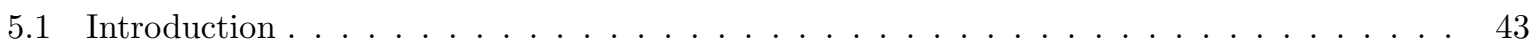

5.2 Pole integration and wave packet correspondence $\ldots \ldots \ldots \ldots$. . . . . . . . . 44

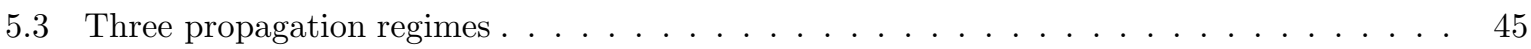

$5.3 .1 \quad$ Laplace's and stationary phase methods . . . . . . . . . . . . . . . . 45

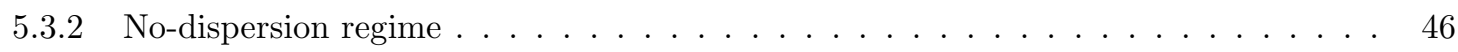

$5.3 .3 \quad$ Transversal-dispersion regime $\ldots \ldots \ldots \ldots \ldots \ldots$

5.3 .4 Longitudinal-dispersion regime $\ldots \ldots \ldots \ldots \ldots \ldots \ldots \ldots$

5.4 Estimates of the dispersion thresholds $\ldots \ldots \ldots \ldots \ldots \ldots \ldots \ldots$

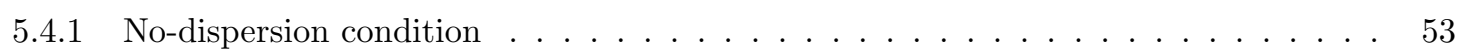

5.4 .2 Dispersion length $\ldots \ldots \ldots \ldots \ldots \ldots \ldots \ldots \ldots \ldots$

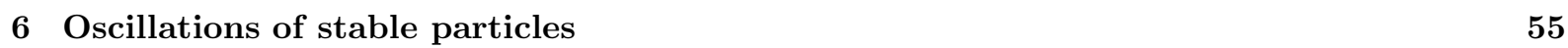

6.1 Oscillations without dispersion $\ldots \ldots \ldots \ldots \ldots \ldots \ldots \ldots \ldots$

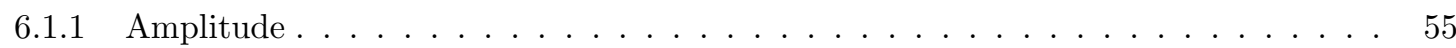

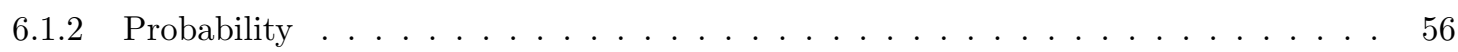

6.2 Oscillations with transversal dispersion $\ldots \ldots \ldots \ldots \ldots \ldots \ldots \ldots \ldots$

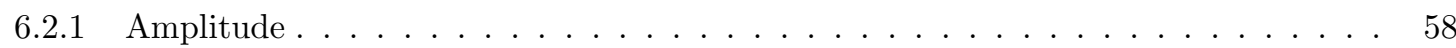

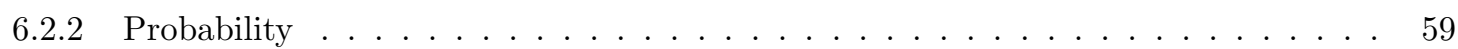

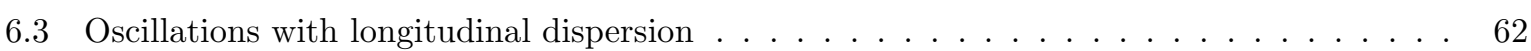

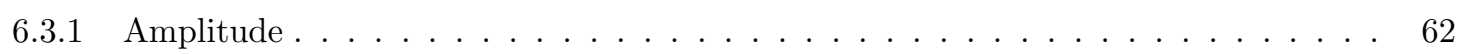

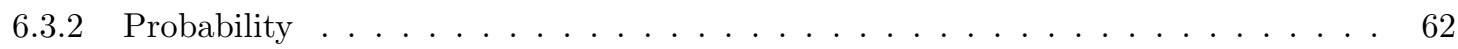

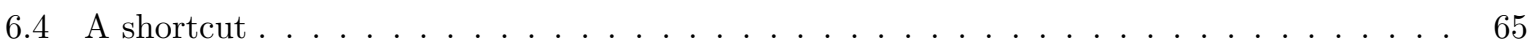

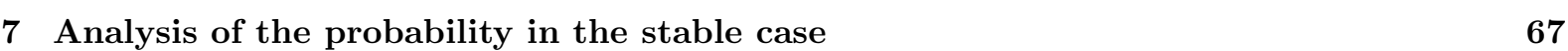

7.1 Oscillations at large distance $\ldots \ldots \ldots \ldots \ldots \ldots \ldots$

$7.1 .1 \quad$ Oscillation formula at large distance $\ldots \ldots \ldots \ldots \ldots$. . . . . . . . . . 68

7.1 .2 Oscillation phase $\ldots \ldots \ldots \ldots \ldots \ldots \ldots \ldots \ldots$

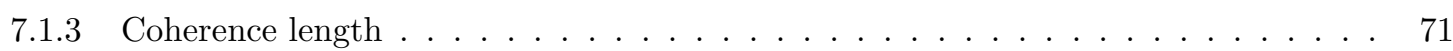

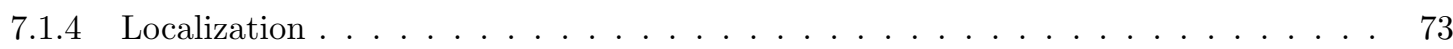

7.1 .5 Energy-momentum conservation $\ldots \ldots \ldots \ldots \ldots \ldots \ldots$

7.2 Oscillations at short distance $\ldots \ldots \ldots \ldots \ldots \ldots \ldots \ldots$

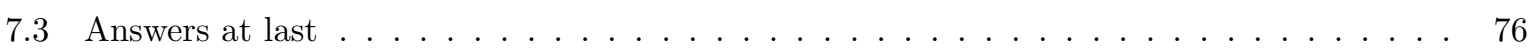

$7.3 .1 \quad$ Existence of a stationary limit $\ldots \ldots \ldots \ldots \ldots \ldots \ldots$

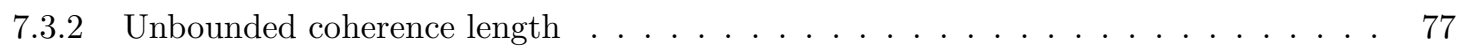

7.3 .3 No mass prefactors at large distance $\ldots \ldots \ldots \ldots \ldots \ldots . \ldots \ldots$

7.3 .4 No plane waves . . . . . . . . . . . . . . . . . . . . . 78

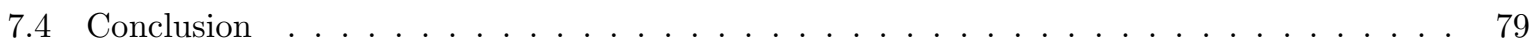




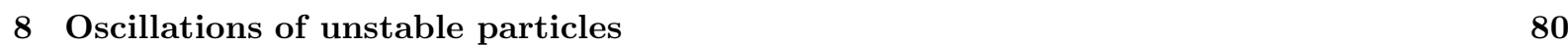

8.1 Nonexponential corrections due to mixing . . . . . . . . . . . . . . . . . . . . . . . . 80

8.2 Oscillation formula for unstable particles . . . . . . . . . . . . . . . . . . . . . . . . . . . . 83

$8.3 \quad$ Wigner-Weisskopf effective mass matrix . . . . . . . . . . . . . . . . . . . . . . . . . . . 86

8.4 Oscillations in the $B^{0} \bar{B}^{0}$ system $\ldots \ldots \ldots$. . . . . . . . . . . . . . . . . . . . . . . . . 87

$\begin{array}{lll}9 & \text { Selected topics } & 90\end{array}$

9.1 Normalization of the transition probability . . . . . . . . . . . . . . . . . . . . . . . . . . 90

9.2 Coherence, incoherence and decoherence . . . . . . . . . . . . . . . . . . . . . . . . . . . . 92

9.2 .1 Incoherent effects . . . . . . . . . . . . . . . . . . . . . . . . 93

9.2 .2 Coherent effects . . . . . . . . . . . . . . . . . . . . 94

9.3 Unstable source . . . . . . . . . . . . . . . . . . . . . . . . . . . . 96

\begin{tabular}{ll}
\hline 10 Correlated oscillations & 97 \\
\hline
\end{tabular}

10.1 Introduction . . . . . . . . . . . . . . . . . . . . . . . 97

10.2 Factorization . . . . . . . . . . . . . . . . . . . . . . . . . . . 99

10.3 Energy-momentum correlation at the source . . . . . . . . . . . . . . . . . . . . . . . 100

10.4 No recoil oscillations . . . . . . . . . . . . . . . . . . . . . . . . . . . . . . 102

10.5 Oscillations of correlated mesons . . . . . . . . . . . . . . . . . . . . . . . . . . . . 104

$\begin{array}{ll}11 \text { Summary and outlook } & 106\end{array}$

\begin{tabular}{ll}
\hline Appendix & 109 \\
\hline
\end{tabular}

References

\section{List of Figures}

$1 \quad$ Decoherence due to a wave packet width $\sigma_{x}$ larger than the oscillation length. . . . . . . . 20

2 Decoherence due to the non-overlapping of the different mass eigenstates wave packets. . . 20

$3 \quad$ Propagation of a particle between a source and a detector, centered in $x_{P}$ and $x_{D}$. . . . . 30

4 Laplace's method is preferable if the phase varies slowly (upper drawing), whereas the method of stationary phase is preferable if the phase varies rapidly (lower drawing). The overlap function (resp. the cosine of the phase) is indicated on both drawings by a continuous (resp. dotted) line. . . . . . . . . . . . . . . . . . . . . . . 46

$5 \quad$ The propagation range can be divided into three regimes according to the dispersion. . . . 54

6 The probability of detection is maximal within a cone at large distance and within a cylinder at short distance. . . . . . . . . . . . . . . . . . . . . . 69

$7 \quad$ In the transversal-dispersion regime (II), the coherence length is due to the non-overlap of wave packets, whereas it is due in the longitudinal-dispersion regime (III) to their dispersion (the coherence length was not computed in the no-dispersion regime). . . . . . . . . . . . 72

8 In the no-dispersion and transversal-dispersion regimes (I and II), the localization condition is due to the initial spread of the wave packet, whereas it is due in the longitudinaldispersion regime (III) to their non-overlap. . . . . . . . . . . . . . . . . . . . . . . . . . 74

$9 \quad$ Integration contour if $b<M_{1}<m<M_{2}$. . . . . . . . . . . . . . . . . . . . . . . . . . . . 111

10 Integration contour if $M_{1}<b<m<M_{2}$. . . . . . . . . . . . . . . . . . . . . . . . . 113 


\section{Introduction}

Nearly fifty years ago, the $\theta$ puzzle led Gell-Mann and Pais [134] to discover that interaction (or flavor) eigenstates need not coincide with particles having well-defined masses and lifetimes. The concept of flavor-mixing was born. Pais and Piccioni [219] went one step further and suggested that the propagation of a flavor eigenstate, as a coherent superposition of mass eigenstates, leads to the partial conversion of the initial flavor into other flavors. The flavor-mixing transition probability oscillates in space with a wavelength depending on the mass differences. The theory of flavor-mixing and particle oscillations was soon vindicated in a series of experiments on the kaon system [190, 114, 148, 171]. Similar oscillations were observed in 1987 with $B$ mesons [13].

By analogy, Pontecorvo proposed in 1957 the hypothesis of neutrino-antineutrino oscillations [229, 230]. Neutrino flavor-mixing, strictly speaking, was suggested somewhat later [203], and the two-flavor oscillation case, as well as its application to solar neutrinos, was examined at the end of the sixties by Pontecorvo and Gribov [231, 149]. Neutrino oscillations are, however, much more difficult to observe than meson oscillations, because of the small masses and the weak interactions of the neutrinos. They remained for thirty years a theoretical subject (see for example the reviews [66, 65]), with the noteworthy exception of the solar neutrino experiment in the Homestake Mine [98, 97, 93, 34]. In the 90's, the persistent deficit in solar neutrinos (in comparison with the predictions of solar models [34, 40, 95]) observed at Homestake was confirmed by the experiments Kamiokande-II [161, 122], SAGE [1, 2], GALLEX [24, 157, GNO [16] and Super-Kamiokande [123, 126]. Moreover the Sudbury Neutrino Observatory recently announced the detection of a nonelectronic component in the solar neutrino flux [9, 10], the total flux being compatible with the predictions of solar models. All these observations can be explained by neutrino mixing and oscillations [47, 43, 38, 127, 211] On another length scale, the observation of an angular dependence of the atmospheric muon-neutrino flux by the Super-Kamiokande Collaboration 124, 125, 175, 170] gave a huge impetus to the neutrino oscillation hypothesis (note that a global muon-neutrino depletion had already been observed in other atmospheric neutrino experiments 1160, 121, 51, 18, 15]). Preliminary results from the long-baseline experiment K2K 11, 164 confirm these results by showing a depletion in an artificially produced muon-neutrino beam. Finally, controversial evidence for neutrino oscillations has appeared in the laboratory experiment LSND [28, 29] but has not been confirmed (nor totally excluded) by the KARMEN experiment [26, 27, 92].

Particle oscillations are an interference phenomenon which allows the measurement of extremely small quantities, such as masses too small to be measurable by other means (mass differences between conjugate mesons [114, 148, 13] or neutrinos [113, 180]), or CP violation (measured nearly forty years ago in neutral meson systems [91, 77] and yet to be measured in the leptonic sector [103]). Thus, it is a privileged tool in the discovery of new physics. Nowadays, $B$ meson and neutrino oscillations have a huge importance in the determination of the

unknown parameters of the Standard Model and its minimal extension, in particular the mixing matrix elements (including the $\mathrm{CP}$ violation phase) and the neutrino masses. Besides serving as 
a delicate probe in particle physics, particle oscillations are a good testing ground for quantum mechanics. Einstein-Podolsky-Rosen correlations [162, 100, 196] have been studied theoretically [57, 58, 116, 228] as well as experimentally [25] in the neutral $K$ and $B$ systems. Bell inequalities for neutral kaons are the subject of active research [22, 59, 135, 76]. Furthermore, fundamental issues of quantum mechanics are raised by oscillation experiments. The determination of the conditions under which the coherence between the different mass eigenstates is maintained is crucial for the phenomenology of neutrino oscillations [182, 204]. The question of the correlation between neutral mesons, in processes where $B^{0} \bar{B}^{0}$ is included in the final state, is important for an estimate of the oscillation frequency [153].

Considering the practical importance of the oscillations, the theoretical framework describing them should be rock-solid. Surprisingly, the validity of the standard treatment of oscillations, resorting to plane waves in a simple quantum-mechanical model, was not seriously questioned until neutrino oscillations finally gained credibility in the 90's. Two pioneering articles should be noted. First, a quantum field model of kaon oscillations was proposed by Sachs in 1963 [238], but was soon forgotten, until the use of Sachs' reciprocal basis was recently revived for a rigorous quantum-mechanical treatment of meson oscillations [77, 17]. Second, Kayser showed in 1981 that neutrino oscillations are in contradiction with the plane wave assumption of the standard treatment of oscillations, and he proposed to modelize the oscillating particles with wave packets [176]. His model, however, was not widely accepted and numerous articles have continued to appear to this day, discussing the ambiguities of the plane wave standard treatment, such as the proper choice of the frame [201] or the equality of the energies or momenta of the different mass eigenstates 136 .

Although Kayser's wave packet model is a serious improvement on the standard plane wave treatment, it also suffers from serious defects: flavor states are ill-defined [142]; the nonrelativistic limit is problematic if the mass eigenstates are not nearly degenerate [142]; the question about the equality (or not) of the energy of the different mass eigenstates is not answered [155, 248]; the possibility remains of obtaining nonstandard oscillation formulas [245]; the relation between the coherence length, beyond which oscillations vanish, and the conditions of production and detection is vague (problem of the determination of the wave packet size [182]); finally, it is not adequate for the oscillations of unstable particles.

In order to solve these problems, a quantum field treatment of neutrino oscillations, similar to Sachs's model, has been proposed by Giunti, Kim, Lee and Lee [145]. The model consists in treating the oscillating particle as an internal line of a Feynman diagram, propagating between a source and a detector, which are represented by ingoing and outgoing external wave packets. This approach has been followed by other authors, but no agreement has yet been reached on the correct assumptions and calculation methods:

1. There is disagreement on whether the interfering mass eigenstates have the same energy [150] or whether their energy is spread; the existence of propagating wave packets, associated to the mass eigenstates, is also at stake [151. 
2. Whereas oscillations vanish if the 3 -momentum is measured too accurately, it is not clear whether the same thing happens when the energy is perfectly measured 183, 144, 139.

3. Nonstandard formulas for neutrino oscillations have been derived in specific cases, either showing a strong dependence of the flavor-mixing transition probability on the neutrino mass ratios [242], or having a 'plane wave' behavior [163], or leading to oscillations of charged leptons in pion decay [247].

Moreover, this formalism cannot be applied, as it is, to meson oscillations or to unstable neutrinos. The existence of neutrino mass makes indeed not only neutrino mixing possible but also neutrino decay [208, 75, 184, 192]. Although it cannot alone explain the data, neutrino decay has a long history as a possible explanation for the solar neutrino anomaly [36, 222, 39, 55, 54, 5, 6, 202, 90, 41, 169, 42, 50]. Moreover this hypothesis was compatible until recently with atmospheric neutrino data [46, 195, 117, 45, 89, 202], although it now seems ruled out [180]. Neutrino decay has also been discussed with respect to supernovae [119, 193].

In this report, we intend to reconcile the different field-theoretical treatments in order to solve the above problems. Our first aim is to argue in favor of a field theory approach of oscillations. We want to show that it provides, on the one hand, the best available derivation of the oscillation formula and gives, on the other hand, a good explanation of the characteristics of the oscillation process (origin of the oscillations, observability conditions, boundary conditions). Our second aim is to strengthen the phenomenological basis of the neutrino oscillation formula through its unification with the mesonic oscillation formula. We thus extend the formalism to the unstable case, so that the same formula can be applied to $\mathrm{K}$ and $\mathrm{B}$ mesons, to neutrinos and to unstable neutrinos.

In section 2, we discuss the quantum-mechanical approaches, so as to understand the problems addressed in most of the articles and the different solutions adopted in the literature. The existing field-theoretical treatments are reviewed in section 3, and their contradictions are summarized in five questions, which will serve as a guideline in the calculations of sections 0 to 6 ; these questions will be answered in section 7.3 and 10. In sections 4 and 5 , we explain all the assumptions behind the external wave packet model. The relativistic case and the nonrelativistic (but nearly mass degenerate) cases are treated in a unified way, and the oscillating particle is allowed to be unstable.

Oscillations of stable particles are analyzed in section 6. With the aim of reconciling the different points of view in the literature, we discuss different methods of computation, which allow to piece together a complete physical interpretation in section 7 . We shall see that this formalism lends itself to a wave packet interpretation of the oscillating particle and can thus take up the physical insights of Kayser's intermediate wave packet model. The dispersion (or spreading in space-time of the amplitude) is taken into account, as it is important for mesons and might be relevant for solar and supernova neutrinos. We can then show that the existence of the coherence length is due not only to the separation of wave packets, but also to their dispersion. We compute the explicit dependence of the coherence length on the conditions of 
production and detection, to check that it increases without bound with the accuracy of energy measurements. We also show in which sense the oscillation formula can be seen as a superposition of mass eigenstates with the same energy, in order to clear up the misunderstanding of stationary boundary conditions. In addition, we analyze in section 7 the nonstandard oscillation formulas derived within field-theoretical models and we disprove them.

Oscillations of unstable particles are examined in section 8. Nonexponential corrections to mixing are computed for the first time in quantum field theory, and they are shown to be negligible. We clearly state the correspondence between the field-theoretical treatment and the Wigner-Weisskopf and reciprocal basis formalisms. Some more topics are discussed in section 9: the normalization of the transition probability, the size of the external wave packets and the instability of the source. Finally, we examine correlated oscillations (DA $\Phi$ NE, KEKB and BABAR experiments) in section 10, including an analysis of the possible energy-momentum correlation

at the source. In the same framework, oscillations of recoil particles are shown to be nonexistent.

Let us mention four restrictions. First, we shall only consider free propagation in vacuum. Interactions with background matter can have a dramatic effect on mixing, as demonstrated by the Mikheyev-Smirnov-Wolfenstein (or MSW) effect [256, 206, 207, 189]. In practice, the MSW oscillations are washed out by averaging over the energy spectrum [227, 204], so that the question of intrinsic decoherence discussed in the present report is not very important in that case. It is true that a field-theoretical formalism for oscillations with matter interactions should be developed to deal with dense astrophysical environments [234, 232, 86, 106], but this goes far beyond the limits of this review. Second, we shall not explain the nonperturbative quantum field theory of particle oscillations, as proposed by Blasone, Vitiello and co-workers [73, 71]. This approach is indeed of a completely different nature than the perturbative field-theoretical treatments discussed in this report. We shall however comment on their results in section 3.1. Third, we shall not examine exotic mechanisms, such as oscillations of massless neutrinos due to a violation of the Equivalence Principle [235], to resonant spin-flavor precession [12, 44], to flavor-changing (or non-universal) interactions [118] or to dissipation [199, 52] (comparative reviews can be found in [195, 220, 132]). Finally, we consider neither the gravitational effects on oscillations [257, 194] nor the possibility of extra dimensions [109, 99].

\section{Oscillations in quantum mechanics}

We first discuss the concept of particle mixing as it is a requirement for oscillations. We then present the standard derivation of the oscillation formula, stressing the problematic choice of basis for unstable particles, as well as the ambiguities arising in the conversion of an oscillation in time into an oscillation in space. This section ends with a review of the attempts to solve these problems with more sophisticated quantum-mechanical models. 


\subsection{Mixing in quantum mechanics}

The possibility of particle mixing arises from the fundamental principles of quantum theory. The measurement of an observable causes the initial state to jump into an eigenstate of the operator associated to this observable. Thus the act of measurement determines a basis of physical states, made up of all the eigenstates of the corresponding operator. The different bases associated to the different operators are linked by linear transformations; a state expanded in a given basis can be seen as a mixing of the eigenstates of that basis. While one gets used to the quantum superposition principle as long as wave functions correspond to rather abstract properties of a system, it is always a bit shocking to apply it to particles, which are associated in our mind with classical concepts. The two-slit experiment with particles is the best known example, but particle mixing, appearing when an interacting eigenstate is a superposition of different mass eigenstates, is hardly less unsettling.

The textbook case is given by the neutral kaon system: the decay pattern of a $K^{0}$ into pions is well explained by the superposition of two mass eigenstates, a CP-even state decaying quickly into two pions, and a CP-odd state decaying more slowly into three pions. Thus a $K^{0}$ can be seen as a mixing of a $K_{S}$ ( $K$ short) and a $K_{L}$ ( $K$ long), having well-defined masses and widths (the so-called mass eigenstate basis). Whereas pionic decays do not allow to identify the final strangeness $\left(K^{0}\right.$ or $\left.\bar{K}^{0}\right)$, it is possible to do so in the decays of kaons into $\pi e \nu$, with the result that one observes a nonnegligible probability to detect a transition from the initial $K^{0}$ into a $\bar{K}^{0}$. This is the phenomenon referred to as particle oscillation. The flavor-mixing transition probability oscillates indeed with the propagation distance, unless the oscillation is averaged by some mechanism, in which case the transition probability simply follows the exponential decay law for each mass eigenstate.

Particle oscillations can be compared to a 2-slit interference experiment, which requires firstly that several paths must be available to a particle, and secondly that these paths should not be distinguishable by the experimental conditions. In particle oscillations, the different mass eigenstates are the analogue of the different paths, since they are eigenstates of the space-time

evolution operators $\hat{\mathbf{P}}$ and $\hat{H}$. Of course, their other quantum numbers should be equal. The equivalent of the second requirement is that the masses should not be distinguishable by the experimental apparatus. Under these conditions, the probability to observe the propagating particle in a given state, other than a mass eigenstate, oscillates in space (and in time) with a wavelength inversely proportional to the mass difference, because of the interference between the mass eigenstate amplitudes. Thus particle oscillations occur if the initial and final states are not mass eigenstates and if it is impossible to ascertain which mass eigenstate has contributed as an intermediate state. This last assumption implies that mass differences should be much smaller than the typical energies involved in the processes, with the result that the oscillation wavelength is macroscopically large and the particle oscillates over macroscopic distances.

Of course, oscillations are only observable in processes where the propagation distance is of the order of, or much larger than, the oscillation wavelength. Otherwise it is perfectly 
adequate to work with interaction (or flavor) eigenstates, as it has been done until now with neutrinos in accelerator experiments. Note that one often talks about particle oscillations even if the oscillation term is averaged by some mechanism, such as an integration over the energy spectrum, though this situation could be more appropriately called incoherent mixing.

Let us be more precise about the basis pertaining to the oscillation phenomenon. The Hamiltonian should be such that it can be split into a propagation Hamiltonian $H_{\text {propag }}$, describing the free propagation of the particle, and an interaction Hamiltonian $H_{\text {int }}$, describing the interactions producing the propagating particle. These two subsets of the Hamiltonian can be distinguished if there exists a quantum number, called flavor, conserved by $H_{\text {int }}$, but violated by $H_{\text {propag }}$. The appropriate basis for the production of the particle is made up of eigenstates of both $H_{\text {int }}$ and the flavor operator. It is called the flavor basis. The basis relevant to the propagation of the particle is the one in which $H_{\text {propag }}$ is diagonal and is called the propagation basis, or mass basis. This basis does not coincide with the flavor basis, since the flavor operator does not commute with $H_{\text {propag }}$.

The simplest example is given by stable neutrinos, in which case the Hamiltonian $H_{\text {propag }}$ includes the mass matrix, whereas the Hamiltonian $H_{\text {int }}$ includes the weak interactions of the neutrinos with the bosons $Z$ and $W$. The flavor is here the leptonic number, which can be electronic, muonic or tauic and maybe sterile. This number is conserved by the weak interactions, but is violated by a non-degenerate mass matrix which mixes neutrinos of different flavors.

As regards unstable particles, the Wigner-Weisskopf method [191, 172, 209] is used to build a non-Hermitian effective Hamiltonian $H_{\text {propag }}$, with the aim of describing both propagation and decay. $H_{\text {int }}$ includes the interactions involved in the production of the oscillating particles. In the case of neutral $K$ (or $B$ ) mesons, $H_{\text {propag }}$ includes, on the one hand, the degenerate effective mass matrix (due to Yukawa, strong and electromagnetic interactions) and, on the other hand, the weak interactions causing the decay and the flavor-mixing transitions $K^{0} \leftrightarrow \bar{K}^{0}$ (or $B^{0} \leftrightarrow \bar{B}^{0}$ ). $H_{\text {int }}$ is an effective Hamiltonian describing the strong interactions of the mesons; it can be built with chiral perturbation theory. The flavor is here the strangeness (or bottomness) with values \pm 1 , which is conserved by strong interactions but violated by weak interactions. The non-Hermiticity of $H_{\text {propag }}$ means that the neutral meson system does not evolve in isolation, because of its possible decay.

The relationship between the propagation and flavor basis can be written as

$$
\left|\nu_{\alpha}(0)>=\sum_{j} U_{\alpha j}\right| \nu_{j}(0)>,
$$

where $U$ is the matrix diagonalizing $H_{\text {propag }}$, if this Hamiltonian has been expressed from the start in the flavor basis. The states $\mid \nu_{j}(0)>$ are the propagation (or mass) eigenstates, at time $t=0$, with well-defined masses and widths, belonging to the propagation basis, whereas the states $\mid \nu_{\alpha}(0)>$ are the flavor eigenstates.

The properties of the matrix $U$ depend on the Hamiltonian $H_{\text {propag }}$. In the case of a stable oscillating particle, the matrix $U$ arises from the diagonalization of the mass matrix and is unitary. In the case of an unstable oscillating particle, $H_{\text {propag }}$ is necessarily non-Hermitian, as 
explained above, so that the time evolution is nonunitary, i.e. the oscillating particle is allowed to decay. The matrix $U$ diagonalizing that Hamiltonian is generally not unitary, although it can be unitary if some symmetry makes the Hamiltonian normal[3 81.

The computation of an amplitude requires the knowledge of the scalar product values of the eigenstates. In the flavor basis, the following orthogonality properties hold:

$$
<\nu_{\beta}(0) \mid \nu_{\alpha}(0)>=\delta_{\alpha \beta}
$$

since $H_{\text {int }}$ is Hermitian and commutes with the flavor operator. In the propagation basis, $H_{\text {propag }}$ is Hermitian only if the oscillating particle is stable. Thus the scalar product of the flavor eigenstates must be defined by transforming the flavor scalar products (2):

$$
<\nu_{i}(0) \mid \nu_{j}(0)>=\sum_{\gamma} U_{j \gamma}^{-1} U_{\gamma i}^{-1 \dagger}
$$

These scalar products become orthogonal if the matrix $U$ is unitary, which is true if the particle is stable or, more generally, if $H_{\text {propag }}$ is normal. In the example of neutral kaons, the approximation of CP symmetry makes $H_{\text {propag }}$ normal and $U$ unitary. Since the violation of this symmetry is of the order of $10^{-3}$, the right-hand side of Eq. (3) is of the same order in the neutral kaon system. This lack of orthogonality implies that the states $\left|\nu_{i}(0)\right\rangle$ can be normalized in different ways. The normalization factors do not matter as long as they do not appear in the final result. A normalization problem arises however in the case of unstable particles if we try to compute an amplitude involving a mass eigenstate in the initial or final state. For example, the amplitude $<\pi \pi\left|H_{\text {total }}\right| K_{L}>$ depends on the normalization chosen for the state $\mid K_{L}>$. In that case, the normalization ambiguity is of the order of the direct CP violation parameter $\epsilon^{\prime}$, so that it cannot be neglected 61. This problem does not arise in the field-theoretical approach, where the kaons are considered as intermediate states, which are not directly observed.

It must be stressed that the orthogonality properties in the flavor basis are valid only if all mass eigenstates are kinematically allowed [63, 220, 96]. For example, let us suppose that there are four stable neutrinos such that $m_{i}=0$ for $i=1,2,3$ and $m_{4} \gg 1 \mathrm{GeV}$. Under $1 \mathrm{GeV}$, the flavor scalar products become

$$
<\nu_{\beta}(0) \mid \nu_{\alpha}(0)>=\delta_{\alpha \beta}-U_{\alpha 4} U_{\beta 4}^{*}
$$

This difficulty is a first indication of the problems arising in the definition of a flavor basis. Is it really possible to interpret a flavor eigenstate as an observable particle? In quantum mechanics, each stable particle can be associated with an irreducible representation of the Poincaré group, with a given mass and a given spin. In that framework, the flavor eigenstates do not correspond to particles, unless they are degenerate in mass. The problem is not easier to solve in quantum field theory. Whereas creation and annihilation operators of mass eigenstates are well-defined, the transformation (1) does not yield creation and annihilation operators of flavor states that satisfy canonical commutation relations [142]. One could thus wonder whether flavor eigenstates

\footnotetext{
${ }^{3} H$ is normal if $\left[H, H^{\dagger}\right]=0$.
} 
really exist or not. A phenomenological argument in favor of their existence is that, for obvious practical reasons, no one has yet devised an experiment involving only one mass eigenstate of a system of oscillating particles . On the contrary, oscillating particles are produced and detected in a superposition of mass eigenstates formally equivalent to a flavor eigenstate. In that sense, flavor eigenstates are observed, whereas mass eigenstates are not.

The case of an unstable particle differs from the stable case in two respects. First, the decay widths of the propagating eigenstates can vary greatly, allowing the isolation of a nearly pure beam of the longest-lived eigenstate (so the phenomenologist is tempted to declare this state 'observable'). Second, there is no state corresponding to an unstable particle in the Hilbert space of physical states. These particles are best described by S-matrix poles in quantum field theory (so the theoretician is tempted to declare these unstable states 'not observable').

\subsection{Oscillations with plane waves}

Many articles and books give derivations of the oscillation formula with plane wave states (see for example Refs. [66, 208]). In this section and in the next, we examine this method in its fine details, emphasizing subtle points and doubtful assumptions.

Following the rules of quantum mechanics, the transition probability between an initial state of flavor $\alpha$ at time $t=0$ and position $x=0$ and a final state of flavor $\beta$ at time $t$ and position $\mathbf{x}$ is given by

$$
\mathcal{A}_{\alpha \rightarrow \beta}(t, \mathbf{x})=<\nu_{\beta}(0)\left|\exp \left(-i \hat{H}_{\text {propag }} t+i \hat{\mathbf{P}} \cdot \mathbf{x}\right)\right| \nu_{\alpha}(0)>,
$$

where $\hat{H}_{\text {propag }}$ is the Hamiltonian operator and $\hat{\mathbf{P}}$ is the generator of translations. The states $\mid \nu_{\alpha, \beta}(0)>$ are taken to be energy-momentum eigenstates, so that a plane wave treatment will follow. This amplitude can be computed with the help of the change of basis (11):

$$
\begin{aligned}
\mathcal{A}_{\alpha \rightarrow \beta}(t, \mathbf{x}) & =\left\langle\nu_{\beta}(0)\left|\sum_{j} U_{\alpha j} e^{-i \phi_{j}-\gamma_{j}}\right| \nu_{j}(0)>\right. \\
& =<\nu_{\beta}(0)\left|\sum_{j, \rho} U_{\alpha j} e^{-i \phi_{j}-\gamma_{j}} U_{j \rho}^{-1}\right| \nu_{\rho}(0)>
\end{aligned}
$$

where the phase is given by

$$
\phi_{j}=E_{j} t-\mathbf{p}_{j} \cdot \mathbf{x}
$$

and the decay term is defined by

$$
\gamma_{j}=\frac{m_{j} \Gamma_{j} t}{2 E_{j}} .
$$

The symbols $E_{j}, \mathbf{p}_{j}, m_{j}$ and $\Gamma_{j}$ stand for the energy, momentum, mass and width of the mass eigenstate $\mid \nu_{j}(0)>$. The factor $\mathbf{p}_{j} \cdot \mathbf{x}$ is often dropped, either because the mass eigenstate is assumed to be in its rest frame (the time $t$ is then the proper time), or because of an assumption of

\footnotetext{
${ }^{4}$ This might have been possible for neutral kaons, had the CP symmetry been exact in that system, making the propagation basis coincide with the CP basis. Decay channels respecting $\mathrm{CP}(K \rightarrow 2 \pi, 3 \pi)$ would have then allowed a nonambiguous determination of the propagating eigenstate.
} 
equal momenta for all mass eigenstates (the factor $\mathbf{p}_{j} \cdot \mathbf{x}$ then does not appear in the probability). These assumptions cannot be justified and neither will be assumed in the following derivation.

If the particle is unstable, $H_{\text {propag }}$ is an effective Hamiltonian, computed with the WignerWeisskopf method in the rest frame of the decaying particle. Hence, the phase depends on the proper time, $\phi_{j}=m_{j} \tau$, so that a boost is necessary to obtain the expressions (6) and (7) valid in the laboratory frame. However the concept of a rest frame has no meaning in the case of a superposition of different mass eigenstates, which may have different velocities. Thus the choice of the boost is ambiguous because it is not clear whether a common boost should be used for the different mass eigenstates, or whether each mass eigenstate is boosted by a different velocity. This difficulty is another indication of the problems arising in the correspondence between a flavor state and a particle. It will be seen in section 2.3 that the choice of the boost has a big impact on the value of the oscillation length.

With the help of the orthogonality property (2), the amplitude (5) can be rewritten as

$$
\mathcal{A}_{\alpha \rightarrow \beta}(t, \mathbf{x})=\sum_{j} U_{\alpha j} e^{-i \phi_{j}-\gamma_{j}} U_{j \beta}^{-1}
$$

In the case of $K$ and $B$ mesons, the oscillation formula (8) is sometimes written in the following way [210]:

$$
\mathcal{A}_{\alpha \rightarrow \beta}(t, \mathbf{x})=\sum_{i, j} U_{\alpha j} e^{-i \phi_{j}-\gamma_{j}} U_{i \beta}^{\dagger}<\nu_{i}(0) \mid \nu_{j}(0)>
$$

The equivalence of this expression with Eq. (8) can be checked with the scalar products (3).

Another way to obtain the transition amplitude (8) for neutral mesons is to define a reciprocal basis 238, 110, 17, 77, 243. This method consists in defining two mass bases: the kets belong to the in basis and are the right-eigenvectors of the Hamiltonian, whereas the bras belong to the out basis (or reciprocal basis) and are the left-eigenvectors of the Hamiltonian. Their Hermitian conjugate vectors are not used to write amplitudes. The Hamiltonian can then be expressed in a diagonal form:

$$
\hat{H}_{\text {propag }}=\sum_{j}\left|\nu_{j}(0)>_{\text {in }} \lambda_{j \text { out }}<\nu_{j}(0)\right|,
$$

where the $\lambda_{j}=m_{j}-i \Gamma_{j} / 2$ are the complex eigenvalues of the Hamiltonian in the rest frame of the oscillating particle (in the mass-degenerate limit). The new bases are related to the flavor basis by

$$
\begin{aligned}
\mid \nu_{\alpha}(0)> & =\sum_{j} U_{\alpha j} \mid \nu_{j}(0)>_{\text {in }}, \\
<\nu_{\alpha}(0) \mid & =\sum_{j} \text { out }<\nu_{j}(0) \mid U_{j \alpha}^{-1} .
\end{aligned}
$$

With these notations, a kind of orthogonality property is restored:

$$
{ }_{\text {out }}<\nu_{i}(0) \mid \nu_{j}(0)>_{\text {in }}=\delta_{i j}
$$


The oscillation formula obtained with these new bases is identical to Eq. (8), since it is simply another way to decompose $\hat{H}_{\text {propag }}$.

The physical interpretation of the reciprocal basis is not clear and its use is not really necessary, since the same result can be obtained without it. Actually, the reciprocal basis method is a technical trick which is best understood from a field-theoretical viewpoint. After all, the new bases were first introduced [238] in a quantum field theory approach as left- and right-eigenvectors of the propagator representing the oscillating particle. As emphasized by Enz and Lewis [110], they should always appear through Eq. (10), underlining their unphysical and intermediate character. For example, the quantity $\left|\left\langle\pi \pi\left|H_{\text {total }}\right| K_{L}\right\rangle\right|^{2}$ is not an observable, as mentioned in section 2.1.

In the special case of a Hermitian $H_{\text {propag }}, U$ is unitary and $\gamma_{j}=0$. The amplitude then reads

$$
\mathcal{A}_{\alpha \rightarrow \beta}(t, \mathbf{x})=\sum_{j} U_{\alpha j} e^{-i E_{j} t+i \mathbf{p}_{j} \cdot \mathbf{x}} U_{\beta j}^{*} \cdot
$$

This is the expression commonly used for neutrinos, except that the term $\mathbf{p}_{j} \cdot \mathbf{x}$ is often dropped for the reasons mentioned after Eq. (7).

In the general case of a non-Hermitian Hamiltonian $H_{\text {propag }}$, the transition probability is given by the squared modulus of the oscillation amplitude (8):

$$
\mathcal{P}_{\alpha \rightarrow \beta}(t, \mathbf{x})=\sum_{i, j} U_{\alpha i} U_{i \beta}^{-1} U_{\alpha j}^{*}\left(U^{-1}\right)_{j \beta}^{*} e^{-i\left(\phi_{i}-\phi_{j}\right)-\gamma_{i}-\gamma_{j}} .
$$

The particle oscillation phenomenon is manifest in the oscillatory behavior of the interference terms in the transition probability (12), with a phase $\phi_{i}-\phi_{j}$ depending on the point $(t, \mathbf{x})$ of detection.

Regarding antiparticle oscillations, the CPT theorem applied to the amplitude (8) gives the relationship

$$
\mathcal{A}_{\bar{\alpha} \rightarrow \bar{\beta}}(t, \mathbf{x})=\mathcal{A}_{\beta \rightarrow \alpha}(t, \mathbf{x}),
$$

so that the oscillation formula for antiparticles is obtained from Eq. (12) by the exchange of $\alpha$ and $\beta$. Note that $\mathrm{CP}$ violation arises from terms in Eq. (12) breaking the $\alpha \leftrightarrow \beta$ symmetry, since it appears through a difference between $\left|\mathcal{A}_{\bar{\alpha} \rightarrow \bar{\beta}}(t, \mathbf{x})\right|^{2}$ and $\left|\mathcal{A}_{\alpha \rightarrow \beta}(t, \mathbf{x})\right|^{2}$.

\subsection{Time to space conversion of the oscillating phase}

\subsubsection{Standard oscillation phase}

There has been some controversy about the conversion of the oscillation phase $\phi_{i}-\phi_{j}$, appearing in Eq. (12), into a measurable quantity. Since the propagation time is not measured in oscillation experiments o prescription is needed to get rid of the time dependence in the phase difference.

The numerous prescriptions proposed in the literature are somewhat confusing. They can be classified by expanding the phase around an average energy or momentum. Since the oscillating

\footnotetext{
${ }^{5}$ However time measurements are important according to Okun 217] in an experiment performed at IHEP (Serpukhov) 23].
} 
particle is on-shell, the energy $E_{j}$ can be expressed in function of the momentum $p_{j}$. The phase $\phi_{j}$ can then be expanded around an average momentum $p$, not very different from $p_{j}$ or $p_{k}$, and an average mass $m$. Although the mass $m$ can be very different from $m_{j}$ or $m_{k}$ in the ultra-relativistic case, the expansion is correct as long as the mass difference $\delta m_{j}^{2}=m_{j}^{2}-m^{2}$ is small with respect to the energy. The momentum difference $\delta p_{j}=p_{j}-p$ is expected to be of the same order than $\delta m_{j}^{2}$. In one spatial dimension, the expansion of the phase reads, to first order in $\delta m_{j}^{2}$ and $\delta p_{j}$,

$$
\begin{aligned}
\phi_{j} & =\sqrt{p_{j}^{2}+m_{j}^{2}} t_{j}-p_{j} x \\
& =E t-p x+\frac{\delta m_{j}^{2}}{2 E} t+(v t-x) \delta p_{j}+E \delta t_{j},
\end{aligned}
$$

with the average energy and velocity defined by $E=\sqrt{p^{2}+m^{2}}$ and $v=p / E$, respectively. A different time $t_{j}$ has been allowed for each mass eigenstate, and expanded around an average time $t$, with $\delta t_{j}=t_{j}-t$. Of course, the following arguments will only be correct to first order in $\delta m_{j}^{2} / 2 E$, but it is useless to argue about further orders in a flawed approach such as the plane wave treatment.

All prescriptions leading to the standard oscillation formula set $\delta t_{j}=0$, i.e. they impose that interference only takes place for equal propagation times (and lengths) for the different mass eigenstates. This equal time prescription has been explicitly stated [181, 177, 178, 201], in reaction against articles proposing different detection times [244, 245]. It has also been legitimated by an equal velocity prescription [245, 101], which is seen to be equivalent to the previous prescription with the help of the classical relation $t_{j}=x / v_{j}$. However the equal velocity condition leads to $\frac{E_{j}}{E_{i}}=\frac{m_{j}}{m_{i}}$, which is very unlikely for neutrinos 218].

Note that imposing equal times $t_{i}=t_{j}$, in the laboratory frame, also means imposing equal proper times $\tau_{i}=\tau_{j}$ if the classical relation $\tau_{j}=\sqrt{t_{j}^{2}-x^{2}}$ is used. The last relation implies that a boost of velocity $v_{j}=x / t_{j}$ is used to go from the rest frame of the mass eigenstate $m_{j}$ to the laboratory frame. Thus the question of the choice of the correct boost boils down to the question of the equality of propagation times in the laboratory frame.

A second prescription is needed in order to obtain the standard oscillation formula. It could be called the classical propagation condition: $v t-x=0$. It imposes that the term $(v t-x) \delta p_{j}$, appearing in Eq. (13), is negligible in comparison with $\delta m_{j}^{2} t / 2 E$. This condition can be weakened to $|v t-x| \ll t$. Since plane waves are delocalized in space-time, this condition cannot be justified without a more sophisticated treatment, for example with wave packets.

These two prescriptions are sufficient to derive the following formula:

$$
\phi_{i}-\phi_{j} \cong \frac{\delta m_{i j}^{2}|\mathbf{x}|}{2|\mathbf{p}|}=2 \pi \frac{|\mathbf{x}|}{L_{i j}^{o s c}},
$$

obtained from a three-dimensional generalization of Eq. (13). The oscillation length $L_{i j}^{o s c}$ is defined by

$$
L_{i j}^{o s c}=\frac{4 \pi|\mathbf{p}|}{\delta m_{i j}^{2}},
$$


where $\delta m_{i j}^{2}=m_{i}^{2}-m_{j}^{2}$ is assumed to be positive. The classical propagation condition also justifies the substitution $t \rightarrow E|\mathbf{x}| /|\mathbf{p}|$ in $\gamma_{i, j}$ (see Eq. (7)).

The transition probability (12) together with the phase (14) form the standard oscillation formula used to fit the experimental data (see [154] for an application to two-flavor neutrino oscillations). Of course, the probability (12) should first be averaged over the energy spectrum and over the region of production and detection (see section 9.2.1). If the oscillation is completely washed out by these averaging procedures, the transition probability (12) can be simplified by the substitution $e^{-i\left(\phi_{i}-\phi_{j}\right)} \rightarrow \delta_{i j}$.

Although the equal time prescription and the classical propagation condition are sufficient to obtain the standard oscillation phase (14), additional prescriptions leading to the same result are commonly found in the literature:

1. The equal momentum prescription is the most common: $\delta p_{i}=\delta p_{j}=0$. As seen above, this assumption is not necessary. Moreover, it is impossible to impose experimental conditions such that the momentum uncertainty is zero, since the oscillations are destroyed by a momentum measurement more accurate than the mass difference. Thus, this prescription is groundless.

2. The equal energy prescription has been recently advocated by Lipkin: $\delta E_{i}=\delta E_{j}=0$ [197]. It has the advantage of avoiding the classical propagation condition since it leads to $\delta p_{j}=-\delta m_{j}^{2} / 2 p$, so that the time dependence completely drops from the phase difference $\phi_{j}-\phi_{k}$ (at least if the equal time prescription is assumed), and the standard oscillation phase (14) is directly obtained.

In principle, oscillation experiments are feasible with a zero energy uncertainty, since spatial oscillations are not expected to vanish in that case (note that a quantum field treatment is necessary to prove it). In practice, the energy uncertainty is far from being negligible and is often of the same order of magnitude as the momentum uncertainty. Thus the equal energy prescription is only justified if an extremely small uncertainty on the energy is imposed by the physical properties of the process itself. Do we have any theoretical reason to expect that this uncertainty is smaller than the mass difference? No convincing arguments supporting that assumption have been given until now. For example ${ }^{6}$, Lipkin computes the energy-momentum uncertainties $\delta p_{K}, \delta E_{K}$ of the kaon in the process $\pi p^{-} \rightarrow \Lambda K^{0}$ [198]. If the proton is at rest in a lattice, its momentum uncertainty, due to the Debye temperature of the crystal, can be estimated at $\delta p_{p} \sim 10^{3} \mathrm{eV}$. Lipkin estimates $\delta p_{K}$ to be of the same order of magnitude, whereas he neglects $\delta E_{K} \sim\left(\delta p_{p}\right)^{2} / m_{p}$. However $\delta E_{K}$ is still much larger than the mass difference $\delta m_{K} \sim 10^{-6} \mathrm{eV}$, which is the most important mass scale in the experiment. Moreover the pion momentum uncertainty

\footnotetext{
${ }^{6}$ Lipkin gives at least three reasons for the equality of energies: the strict energy conservation discussed here [197, 198, the flavor-energy factorization 155, 198] and the stationarity resulting from a time average 197, 198]. The last two arguments are discussed in section 2.5.
} 
gives a first order contribution to $\delta E_{K}$ and should not be neglected. Even if one has shown that $\delta E \ll \delta p$, there is a long way to go to show that $\delta E \ll \delta m$.

Another argument against the equality of energies is that it holds only in one particular frame. For example, if the energies of the different mass eigenstates are equal in the decay of a pion at rest $(\pi \rightarrow \mu \nu)$, the energy difference becomes approximately equal to the momentum difference if the pion is relativistic [136]. Thus the equal energy prescription should be shown to be true in the laboratory frame for any experimental conditions.

However there is a much more reasonable way of looking at the equal energy prescription. It consists in seeing it as the result of a time average washing out the interference between wave packet components having different energies. There is then no need to prove that the energy uncertainty is zero. This argument will be considered in section 2.5 .

3. Energy-momentum conservation at the production: first proposed by Winter [255], this recurring prescription [75, 146, 201, 244, 245, 140, 136, 252] allows to compute explicitly $\delta p_{j}$. It has often been used to show that neither the momenta nor the energies of the different mass eigenstates are equal. In the example of the pion decay at rest $(\pi \rightarrow \mu \nu)$, the energies and momenta of the muon and neutrino can be computed exactly if the energymomentum of the pion is perfectly known. However this knowledge is usually not available: when the energy-momentum spread of the source is much larger than the mass difference $\delta m_{i j}^{2}$, it is meaningless to compute the exact values of the energies and momenta to order

$\delta m_{i j}^{2}$. A more detailed examination of this question requires wave packets instead of plane waves, or even better, quantum field theory.

\subsubsection{Non-standard oscillation phase}

Controversial prescriptions leading to nonstandard oscillations formulas involve different propagation times $\delta t_{i} \neq \delta t_{j} \neq 0$ or, equivalently, different proper times $\tau_{i} \neq \tau_{j}$. Let us parametrize $p_{j}$ by

$$
p_{j}=p+(\rho-1) \frac{\delta m_{j}^{2}}{2 p},
$$

where $\rho$ is a dimensionless number of order unity. The corresponding energy and velocity can be written as

$$
\begin{aligned}
E_{j} & =E+\rho \frac{\delta m_{j}^{2}}{2 E} \\
v_{j} & =v+\left(\rho\left(1-v^{2}\right)-1\right) \frac{\delta m_{j}^{2}}{2 p E} .
\end{aligned}
$$

The momenta are equal if $\rho=1$ whereas the energies are equal if $\rho=0$. As explained above, it has also been proposed to determine $\rho$ through energy-momentum conservation at production, leading to a value of $\rho$ of order unity. In any case, the time difference is computed with the help 
of the classical relation $t_{j}=x / v_{j}$ and reads

$$
\delta t_{j}=\left(E^{2}-\rho m^{2}\right) \frac{\delta m_{j}^{2} x}{2 p^{3} E} .
$$

Inserting this value in Eq. (13) and using $t=x / v$, we obtain

$$
\phi_{j}=E t-p x+\left(1+\rho+\frac{1-\rho}{v^{2}}\right) \frac{\delta m_{j}^{2} x}{2 p} .
$$

With the equal momentum prescription $(\rho=1)$, the corresponding oscillation length will be smaller by a factor 2 than the standard value given in Eq. (15) [197. With the equal energy prescription $(\rho=0)$, the oscillation length will be smaller by a factor $1+v^{-2}$ than in Eq. (15). Thus the equal energy prescription may also lead to a nonstandard oscillation length, contrary to what was claimed in [197]. With the energy-momentum conservation prescription, the oscillation length will be smaller than in Eq. (15) by a factor depending on the value of $\rho$, which depends on the energy [244]. These formulas can also be applied to the case of correlated oscillations, such as $\phi(1020) \rightarrow K^{0} \bar{K}^{0}$ or $\Upsilon(4 s) \rightarrow B^{0} \bar{B}^{0}$. In the center-of-mass frame of the resonance, the different time prescription leads to an oscillation length smaller by a factor 2 than the standard value, since the equality $\left|p_{i}\right|=\left|p_{j}\right|$ valid in that frame leads to $\rho=1$ [245].

Another disturbing consequence of the different time prescription is the oscillation of recoil particles, for example $\Lambda$ in $\pi^{-} p \rightarrow \Lambda K^{0}$ [244], or the muon in $\pi \rightarrow \mu \nu$ [246, 247]. This is easily seen by applying Eq. (13) to the recoil particle. Although $\delta m_{j}^{2}=0$ (the recoil particle has only one mass eigenstate), $\phi_{i} \neq \phi_{j}$ because $\delta t_{i} \neq \delta t_{j}$. The oscillation of particles having only one mass eigenstate is unacceptable since it leads to non-conservation of the detection probability of this particle. This is not the case when there are several mass eigenstates, as the sum of the detection probabilities of the different mass eigenstates is always equal to 1 for a given propagation distance.

The treatment of neutrino oscillations in $\pi \rightarrow \mu \nu$ proposed by Field 112 also resorts to the different time prescription. This author claims that the different neutrino mass eigenstates are detected at the same space-time point but are produced at different space-time points. Following Field, the oscillation phase would not only receive a contribution from the neutrino path difference but also from the path difference of the source which decays at different times. Field computes the first contribution with the energy-momentum conservation prescription, which gives for a source at rest an oscillation phase larger than the standard result by a factor 2 (see Eq. (18) with $v=1$ ). The second contribution, which only appears in Field's article, is computed in the same way. The oscillation phase obtained by Field therefore differs from the standard result (14). Field's method also leads to the prediction of muon oscillations because of the use of the different time prescription!.

Another example of the different time prescription can be found in Malyshkin and Kulsrud's analysis [204] of the time variations of the solar neutrino flux, which leads to a result different

\footnotetext{
${ }^{7}$ Field's criticism of wave packets models will be examined in section 2.5 .
} 
from Parke's formula [224]. As we do not consider here oscillations in matter, this new oscillation formula will not be discussed further, although our comments on the different time prescription also apply to that case.

The different time prescription has been strongly criticized by several authors within the plane wave framework [201, 181, 177, 178, 197, 198]. Their argument8], which can be stated as 'interference only occurs between states taken at the same space-time point', does not hold when examined in a wave packet or field-theoretical model. Kiers, Nussinov and Weiss [182, 183] have indeed shown that the coherent character of the detection process allows wave functions at different space-time points to interfere. In particular, a long coherent measurement in time may be used to revive oscillations, even after the mass eigenstate wave packets have completely separated spatially (see section 2.5). The question of the correct time prescription is thus subtler than it seems at first sight. The crux of the matter is to take into account the production and detection processes. In this way, each increment in the phase associated to the propagating particle, due to a slightly different production (respectively detection) point, is cancelled by a decrement in the phase of the wave packet of the source (respectively detector) [140]. For example, this cancellation can be implemented in the intermediate wave packet model (see section 2.5) by computing the total amplitude as an overlap of the propagating wave packet with the source and detector wave packets [139, 137, 138]. Note however that this mechanism is much more natural in a quantum field model such as the external wave packet model (see section 4), where the amplitude is integrated over all possible microscopic production and detection points, with the result that the phase depends only on average (i.e. macroscopic) production and detection points.

In conclusion, neither the equal time prescription nor the different time prescription can be justified in the plane wave approach, although the choice of the prescription has an important effect on value of the oscillation length. Moreover other prescriptions, such as the classical propagation condition and the equal energy prescription, cannot be understood within the plane waves formalism. A wave packet or quantum field treatment is thus inescapable. Let us also insist on the dubiousness of the arguments using energy-momentum conservation. In most cases, they are invalidated by the energy-momentum spectrum of the source: different energy-momentum components of the source can contribute to different mass eigenstates.

\subsection{Problems with the plane wave treatment}

In section 2.3, it has been shown that the plane wave treatment of particle oscillations cannot deal in a satisfactory way with the time dependence of the oscillating phase. Besides, this approach implies a perfectly well-known energy-momentum and an infinite uncertainty on the space-time localization of the oscillating particle. Oscillations are destroyed under these assumptions [176]. On the one hand, the perfect knowledge of the energy-momentum allows to determine which mass eigenstate propagates. On the other hand, the spatial delocalization makes impossible the

\footnotetext{
${ }^{8} \mathrm{~A}$ more convincing (though indirect) argument has been given by Giunti [137]: the use of the different time prescription in the double-slit experiment leads to a wrong interference pattern.
} 
measurement of the oscillation length. A correct oscillation formula should include observability conditions in such a way that the oscillation term vanishes if either

1. the energy-momentum uncertainty is smaller than the mass difference between the interfering mass eigenstates, or

2. the oscillation length is of the same order, or smaller, than the uncertainty on the position of the source or of the detection point of the oscillating particle.

Another kind of problem is not specific to the plane wave treatment, but affects all approaches where the oscillating particle is considered to be directly observable. On the one hand, flavor eigenstates are ill-defined for stable particles, because we do not know how to define creator and annihilation operators of flavor states satisfying canonical (anti)commutation relations [142]. On the other hand, mass eigenstates are ill-defined for unstable particles, since they are in general not orthogonal (except if the Hamiltonian is normal, see section 2.1). The solution to these problems simply consists in considering the oscillating particle as an intermediate state. Actually this stand reflects well the experimental situation, where the oscillating particle is not directly observed. One rather detects the particles in interaction with the oscillating state, both at the source and at the detector. The flavor transition probability should thus be computed with observable particles as initial and final states [187, 142, 145, 237].

Finally, we should mention two other problems regarding unstable particles. These difficulties arise because of the nonrelativistic Wigner-Weisskopf method used to compute the effective Hamiltonian. First, interference between different mass eigenstates is forbidden in nonrelativistic quantum mechanics (Bargmann superselection rule [48, 173, 133]). The argument is the following. The invariance of the Schrödinger equation under Galilean transformations determines the transformation law of a quantum state: it is multiplied by a phase factor depending on the mass and space-time position of the state. Thus different mass eigenstates transform differently, so that the relative phase in a superposition of such eigenstates is not conserved under Galilean transformations. Therefore a coherent superposition of different mass eigenstates is forbidden. Second, unstable particles cannot be consistently described in nonrelativistic quantum mechanics for the same reason at the origin of the Bargmann superselection rule: transitions between different mass eigenstates are forbidden [173]. Unstable states cannot be considered as asymptotic states. Thus they do not appear in the Hilbert space of physical states and must be treated in quantum field theory where they appear as complex poles of the full propagator [253].

\subsection{Intermediate wave packets and other improvements}

Some of the problems of the plane wave treatment are solved by the intermediate wave packet model, in which a wave packet is associated with each propagating mass eigenstate. Note that this model is usually discussed with respect to neutrinos, i.e. in the relativistic limit. Nussinov was the first to put forward the existence of wave packets as the cause of a coherence length beyond which oscillations vanish [216]. Oscillations with wave packets were then studied in 


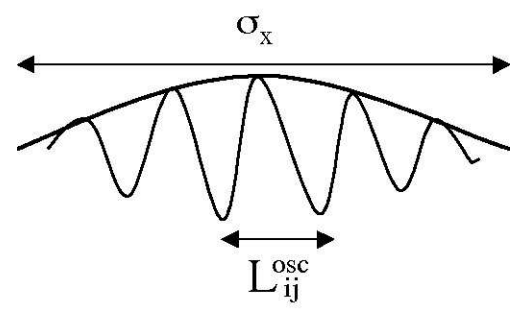

Figure 1: Decoherence due to a wave packet width $\sigma_{x}$ larger than the oscillation length.
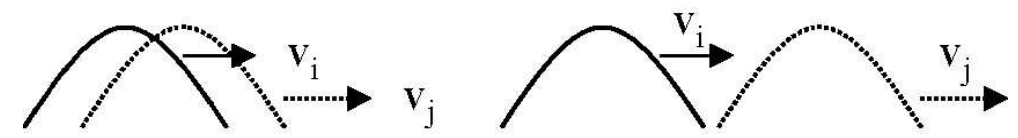

Figure 2: Decoherence due to the non-overlapping of the different mass eigenstates wave packets.

detail by Kayser [176]. The oscillation formula was later explicitly computed with Gaussian wave packets by Giunti, Kim and Lee 141, 139, 137.

The intermediate wave packet model shows that oscillations vanish if $\sigma_{x} \gtrsim L_{i j}^{o s c}$, i.e. if the uncertainty over the position is larger than the oscillation length (Fig. 1). For minimal uncertainty wave packets, this condition can be rewritten as $\sigma_{p} \lesssim \delta m_{i j}^{2} / 2 p$, i.e. oscillations are forbidden if the momentum spread of the wave packets is smaller than the mass difference between the interfering eigenstates. Thus oscillations are destroyed by energy-momentum measurements aiming to determine which mass eigenstate propagates.

The other main result of the model is the existence of a coherence length beyond which oscillations vanish [216, 176, 141]. Its usual explanation is that wave packets associated to different mass eigenstates have different group velocities. Hence, wave packets progressively separate, and interference disappears when they do not overlap anymore (Fig. 2). We shall see in section 7.1 .3 that dispersion is also at the origin of the coherence length.

In the intermediate wave packet model, the classical propagation condition, $|v t-x| \ll t$, is automatically implemented by the space-time localization of the wave packet. However the question of the equal or different time prescription is not solved, since the times of production and detection are not specified by the formalism. As regards the question of the equality of energies or momenta, it is in principle no longer relevant, since the energy-momentum is spread out. In fact, the oscillation length depends only on the zeroth order values (in a $\delta m_{i j}^{2}$-expansion) of the average energies and momenta, because of the cancellation between the time and space parts of the phase (see Eqs. (13) and (16) with $p_{j}$ equal to the average momentum). The time dependence of the probability is dealt with by averaging over time [141]. It is thus not necessary to worry about the equality of energies or momenta.

Nevertheless there is still much argument about the equality of the energy spectra, or the equality of the average energies, momenta or velocities. For example, Grossman and Lipkin 
155] imposed a same energy spectrum for all mass eigenstates, so that the probability of finding a particle with the wrong flavor vanishes for all times at the position of the source. It can be objected that this boundary condition is automatically satisfied without imposing an identical energy spectrum, since the probability to detect the oscillating particle at the source becomes negligible once the wave packet has left it. Equality of average velocities has been proposed by Takeuchi, Tazaki, Tsai and Yamazaki [250, 251], but is very unlikely for neutrinos, as noted by Okun and Tsukerman [218], because the ratio of the average energies $E_{i} / E_{j}$ should then be equal to the mass ratio $m_{i} / m_{j}$.

Actually there is another way of looking at this question. Starting with arbitrary wave packets, it is simple to check that interference occurs only between wave packet components with the same energy, at least if the oscillation process is strictly stationary. This line of thought has been advocated by Sudarsky et al. [249] (within a field-theoretical model), by Lipkin [197, 198] (with wave packets) and by Stodolsky [248] (within a density matrix formalism). Let us explain it with the intermediate wave packet model. In one dimension, the wave function corresponding to the mass eigenstate $m_{i}$ can be written as

$$
\psi_{i}(x, t)=\int d E A_{i}(E) e^{-i E\left(t-t_{0}\right)+i k_{i}\left(x-x_{0}\right)},
$$

where $k_{i}=\sqrt{E^{2}-m_{i}^{2}}$ and $\left(t_{0}, x_{0}\right)$ are the coordinates of the source. The flavor-mixing transition probability is a linear superposition of terms $\psi_{i}(x, t) \psi_{j}^{*}(x, t)$. If the time of emission is unknown, the probability is averaged over $t_{0}$, so that it becomes a superposition of terms like

$$
\int d t_{0} \psi_{i}(x, t) \psi_{j}^{*}(x, t)=2 \pi \int d E A_{i}(E) A_{j}^{*}(E) e^{i\left(k_{i}-k_{j}\right)\left(x-x_{0}\right)} .
$$

This equation makes clear that interference occurs only between wave packet components with the same energy. The time-averaged transition probability in the wave packet model is equivalent to an incoherent sum over energy eigenstates ('incoherent' means a sum in the probability, not in the amplitude). Eq. (19) can thus be seen as a sum over plane waves of energy $E$ and momentum $k_{i}$, weighed by the factor $A_{i}(E) A_{j}^{*}(E)$. The equivalence between a wave packet and a plane wave decomposition of a stationary beam was already observed by Dicke and Wittke [104] in connection with electron-interference experiments, and by Comsa [94 in connection with neutron-interferometry. The same issue was recently raised by Kiers, Nussinov and Weiss [182] and by Stodolsky [248, this time in connection with neutrinos.

Of course the time integral in Eq. (19) only yields a delta function if the time interval is infinite, i.e. if the process is strictly stationary. In practice, there is always some available information on the time of emission. If the time integral is limited to the interval $\Delta T$, the delta function becomes a narrow peak of width $1 / \Delta T$, which can be neglected as long as it is small in comparison with the mass difference $\delta m_{i j}^{2} / 2 E$. This condition can be written as $\Delta T \gg T_{i j}^{o s c}$, where $T_{i j}^{o s c}$ is the oscillation time.

A few more results have been obtained with wave packets. Kiers, Nussinov and Weiss have used the equivalence between wave packets and plane waves, explained above, to show that it 
is always possible to increase the coherence length by more accurate energy measurements at the detector [182]. After Giunti and Kim 139] showed how to take into account the detector's momentum uncertainty in the intermediate wave packet model, Giunti [137] did the same with the detector's energy uncertainty, so as to reproduce Kiers, Nussinov and Weiss' prediction. Wave packets have also been applied by Nauenberg to study correlated oscillations of neutrinos or mesons [212], and to the propagation of neutrinos in matter [143, 225]. Finally, it is worth mentioning that the wave packet approach is explained in great detail in Kim and Pevsner's book [185], is discussed by Fukugita and Yanagida [128] and has been reviewed by Zralek [258].

The intermediate wave packet model has been recently discussed by De Leo, Nishi and Rotelli [102], who recover the standard oscillation formula under two conditions. The first condition, which they call 'minimal slippage', is equivalent to the existence of the coherence length discussed above, i.e. mass eigenstate wave packets should overlap at detection otherwise there is decoherence. The second condition, which they call 'non instantaneous creation' or 'pure flavor creation event-wise', means that interference occurs between wave packet components corresponding to different initial wave packet points, so that the standard oscillation phase (14) is obtained. This mechanism, which was already explained by Giunti and Kim [140] (see end of section 2.3.2), is automatically included in quantum field treatments where the oscillating particle is coupled to a source and detector (see section 1 ). In the same article, De Leo, Nishi and Rotelli argue that the oscillation formula might depend on the wave packet shape, for example if the wave packet is a superposition of two well-separated Gaussian peaks, each having its own phase factor. In that case, there would be a succession of coherence/decoherence/coherence/decoherence regions as the mass eigenstates wave packets shift one with respect to the other. Moreover, the constant phase difference between the two Gaussian peaks crops up in the oscillation phase in the second coherence regime. While surprising phenomena indeed arise for such special wave packet shapes, it seems difficult to set up an experiment in which a specific wave packet shape is maintained for all the particles within the produced flux. In practice we should average over all wave packet shapes, with the result that only the typical width of the wave packet will have an effect on the final oscillation formula. All other effects due to the different shapes of the wave packets cancel each other out. Besides, the analysis of oscillations in a faraway region (such as the second coherence regime mentioned above) should take into account the fact that dispersion wipes out oscillations at large distances (see section 7.1.3).

The representation of the oscillating particle by a minimal uncertainty wave packet has been criticized by Field [112], who claims that that oscillating particles follow classical space-time trajectories and that the oscillation phase is due to the propagation time difference between different mass eigenstates (see section 2.3). One of his main arguments is that the neutrino momentum spread in $\pi \rightarrow \mu \nu$ is of the order of decay width $\Gamma_{\mu}$ of the muon, so that neutrino oscillations should be completed suppressed by decoherence in wave packet models. Moreover, the Heisenberg relation is violated if one admits that the neutrino spatial uncertainty is of the order of the $\mathrm{cm}$. These arguments do not hold since the neutrino momentum spread reflects the momentum uncertainties of the pion source and of the emitted muon, which are much larger 
than $\Gamma_{\mu}$. Finally, Field criticizes models where the neutrino wave packet is coupled to a wave packet source, by claiming that the initial quantum state is not the same for all amplitudes, so that interference is forbidden. This criticism is not justified, since it makes no sense to cut the source wave packet into disjointed parts which are then interpreted as different initial states.

Although they solve some of the problems of the plane wave approach, intermediate wave packets are not satisfying for many reasons:

1. The question of the existence of a Fock space for the weak eigenstates remains.

2. Oscillating particles are not, and cannot be, directly observed. It would be more convincing to write a transition probability between observable particles, involved in the production and detection of the oscillating particle.

3. The wave packet shapes, sizes and normalizations are arbitrary. In particular, the amplitudes of production and detection are not taken into account. This is not a problem in the relativistic limit or for nearly degenerate masses, in which cases these amplitudes can be factorized from the sum over the mass eigenstates. However this factorization is not possible for nonrelativistic particles (unless they are nearly degenerate in mass).

4. The coherence length depends on the difference between the group velocities of the wave packets, the exact values of which are unknown in the nonrelativistic case?. There is no reason to believe that the group velocities could be determined, to order $\delta m_{i j}^{2} / 2 p$, by energy-momentum conservation at the source, contrary to what is claimed in [141].

5. The coherence length increases with the precision of the energy-momentum measurements at the source and at the detector. Whereas the momentum precision is limited by the condition $\sigma_{p} \gtrsim \delta m_{i j}^{2} / 2 p$, there is, a priori, no such limitation on the energy accuracy and, therefore, no bound on the coherence length. Although it has been claimed [139] that such a bound exists, the intermediate wave packet model cannot settle that question, because the energy uncertainty has to be inserted by hand into the model.

6. Experiments measure a particle flux, not a particle density. The transition probability must thus be converted to a flux density, involving inverse velocity factors. These enhance nonrelativistic mass eigenstates and strongly skew the probability [141]. This effect is due to the neglect of the amplitudes of production and detection and to an analysis restricted to one dimension.

7. Although it has not been controversial in the wave packet model, why should the times appearing in the different wave packets be identical? A justification of this equal time prescription is necessary to rule out the nonstandard oscillation formulas discussed in section 2.3 .

\footnotetext{
${ }^{9}$ Eq. (17) can be interpreted as giving the group velocity of a mass eigenstate wave packet. In the relativistic limit $(v \rightarrow 1)$, the $\rho$-dependence drops from this equation, so that the velocity can be determined up to order $\delta m_{i j}^{2} / 2 p$ without needing to know $\rho$.
} 
8. It is not possible to analyze in the wave packet model the corrections to the oscillation probability due to the possible instability of the propagating particle.

9. The influence of the source instability on the observability of the oscillations cannot be studied in that framework.

A quantum-mechanical model of neutrino oscillations solving the first three problems has been put forward by Rich [237]. This author considers the oscillating particle as an intermediate state and computes the probability transition between initial and final observable states with second-order time-dependent perturbation theory of standard quantum mechanics. Since this model takes into account the production and detection processes, it has the other advantage of not requiring the equal time prescription. The spirit of Rich's model is similar to the stationary boundary condition models resorting to field-theoretical methods (see section 3.1), but it has the disadvantage of being nonrelativistic.

Ancochea, Bramon, Muñoz-Tapia and Nowakowski have tried to solve the difficulty of converting the probability density for neutral kaons into a flux [21]. In their nonrelativistic wave packet model, they construct flavor probability currents associated to a Schrödinger equation. A problem arising in this approach is that the flavor currents are not conserved [258]. Moreover, no one knows how to extend this method to the relativistic case.

Sassaroli has proposed an hybrid model for neutrino oscillations, going half-way to quantum field theory, in which a coupled system of two Dirac equations is quantized [239]. However boundary conditions cannot be applied consistently, unless lepton flavor wave functions are considered as observable and the relativistic limit is taken. This difficulty was already noted by Giunti, Kim and Lee [142].

The review of intermediate wave packet models would not be complete without mentioning its latest and most sophisticated version, as proposed by Giunti [138]. Instead of representing the oscillating particle by a superposition of arbitrary wave packets, Giunti computes the form of the intermediate wave packet from basic principles. More precisely, it consists in creating the intermediate wave packet from quantum field interactions between the external wave packets involved in the production process of the oscillating particle. The wave packet then evolves in space-time, before interacting with quantum field interactions at the detector. This interacting wave packet model is very close in method, spirit and results to the external wave packet model at the core of the present report. It will be further discussed in section 5.2.

\section{Oscillations in quantum field theory}

\subsection{Review of the literature}

Few authors deny that the most rigorous treatment of oscillations is done in the quantum field theory framework. However, although the quantum field computations in the literature all reproduce in some limit the naive quantum-mechanical formula given by Eqs. (12) and (14), there is not yet an agreement in which respect they differ from the naive formula. There are 
two reasons for the lack of agreement between the existing quantum field derivations of the oscillation formula: first, the different authors use different physical assumptions, and second, they use different approximation schemes to compute the transition probability.

The field-theoretical approach to particle oscillations is quite old. Already in 1963, Sachs 238] applied S-matrix methods to neutral kaon interferencest with Jacob [166] for unstable particles. Let us call this model, as applied to non-oscillating particles, the Jacob-Sachs model. Its application to systems of mixed particles will be called the external wave packet model. In this model, the particle to be studied is represented by its propagator; it propagates between a source and a detector, where wave packets representing the external particles are in interaction. Much later, Sudarsky, Fischbach, Talmadge, Aronson and Cheng 249 studied the influence of a spatially varying potential on the neutral kaon system. They resort to a one-dimensional model similar to Sachs' but do not specify the contour of integration, so that the finiteness of their final expressions is not guaranteed. Neither Sachs nor Sudarsky et al. studied the observability conditions of oscillations. Another simplified model was proposed by Beuthe, López Castro and Pestieau [61], with the aim of modelizing experiments at CPLEAR and DA $\Phi$ NE. Their model is not satisfying since they use external wave packets localized in time but not in space, which does not correspond to actual experiments.

As regards neutrinos, Kobzarev, Martemyanov, Okun and Shchepkin analyzed neutrino oscillations with a bare-bones quantum field model [187]: the source and detector are infinitely heavy nuclei, so that the propagation distance is perfectly known, whereas the propagation time is left undetermined. No constraints on oscillations are discussed in that article. Next came an important article by Giunti, Kim and Lee [142], showing that it is impossible to build a Fock space for flavor states, because the mixing of the ladder operators for mass eigenstates does not yield flavor ladder operators satisfying canonical (anti)commutation relations. This observation strikes a blow to the quantum-mechanical wave packet approach, which should not be mistaken for the quantum field model with external wave packets, as it is sometimes the case in the literature. Nonrelativistic corrections to the neutrino propagation are explicitly computed in Ref. [142] in a few examples.

In a pioneering article, Giunti, Kim, Lee and Lee [145] studied neutrino oscillations within a Gaussian external wave packet model. They derive a localization condition (no oscillations if $L^{o s c} \lesssim \sigma_{x}$ ) and a coherence length beyond which oscillations vanish. These conditions agree with those obtained in the quantum-mechanical picture [216, 176, 141]. A later paper by Giunti, Kim and Lee [144] contains essentially identical results as in Ref. 145], but with more generality. Cardall used the same model [85], paying greater attention to the normalization of the event rate and to the spin structure. A model close in spirit to the external wave packet model was proposed by Kiers and Weiss [183]. These authors couple the oscillating neutrino with localized

\footnotetext{
${ }^{10}$ Quoting Sachs, from 238]: 'The question of whether it is the momenta or the energies of these particles that are to be taken equal in the treatment of interference phenomena has often been raised. [Our] method provides a clear answer to this question since it is based on an analysis of the phenomena in terms of wave packets'. Nearly 40 years after, new articles continue to appear, wondering about the equality of energies or momenta.
} 
source and detector, which are idealized by oscillators. They show how the coherence length increases with the energy precision at the detector. Note that they had already predicted this phenomenon using elementary quantum mechanics [182].

Another kind of model arises from the use of stationary boundary conditions, leading to a unique value for the energy of the oscillating particle. The simplest example is the Kobzarev et al. model mentioned above 187. Going a bit further, Grimus and Stockinger proposed a model with external particles represented either by bound states or by plane waves. They obtain a localization condition but no coherence length. In two other papers, Grimus, Mohanty and Stockinger [151, 152] studied the influence of an unstable source on the oscillations, with the model of the previous paper modified by a Wigner-Weisskopf approximation for the unstable source. Ioannisian and Pilaftsis analyzed neutrino oscillations [163] within a scalar version of the Grimus-Stockinger model. They claim to have found a novel form of neutrino oscillations at short distance, which they call 'plane wave oscillations'. Stationary boundary conditions were also used by Cardall and Chung [87] to study the MSW effect in quantum field theory. Note that a quantum field derivation of the MSW effect has been proposed by Mannheim [205], who represents the interaction with matter with an effective potential, whereas this potential has been derived with finite temperature field theory by Nötzold and Raffelt [214] and by Pal and Pham 223.

Campagne 84] studied a neutrino source decaying in flight, such as a relativistic pion, with field-theoretical methods. He sidesteps external wave packets by limiting arbitrarily the interactions regions with the help of step functions, but it is only a trick to replace complicated interactions at the source and detector by simple stationary boundary conditions. Decay in flight has also been studied by Dolgov [105], within a simplified external wave packet model modified by a Wigner-Weisskopf approximation.

Another approach (source-propagator models) consists in using the propagator in configuration space coupled to a source but not to a detector. Srivastava, Widom and Sassaroli chose this method to modelize correlated oscillations of two kaons [245] and neutrino oscillations [247]. Since external wave packets are absent, time-space conversion problems cannot be avoided and lead to nonstandard oscillation lengths or recoil oscillations (see section 2.3). Shtanov also used a source-propagator model, and claims to have found a strong dependence of the oscillation formula on the neutrino masses if the source and detector are very well localized in space-time [242.

In a completely different line of thought, Blasone, Vitiello and other researchers have attempted to define a Fock space of weak eigenstates and to derive a nonperturbative oscillation formula. The main results of these studies are summarized in [72]. Note that a previous formalism developed in [73, 14, 67, 158] lead to an oscillation formula not invariant under reparametrization [129, 74, 130, 69] and was replaced by a more satisfying theory in [71, 68], which was further developed in [72, 167, 168, 70]. This new theory aims at defining flavor creation and annihilation operators, satisfying canonical (anti)commutation relations, by means of Bogoliubov transformations. As a result, new oscillation formulas are obtained for fermions and 
bosons, with the oscillation frequency depending surprisingly not only on the difference but also on the sum of the energies of the different mass eigenstates. Apart from the speculative nature of the enterprise, the drawbacks of the approach are the dependence on time, not on space, of the oscillation formula (Lorentz covariance is broken), as well as the lack of observability conditions. Although these studies are very interesting from a fundamental point of view, it is not obvious whether the new features of the Blasone-Vitiello oscillation formulas are observable in practice. Since these new oscillation formulas tends to the standard oscillation formula (12) in the relativistic limit or if the mass eigenstates are nearly degenerate, we can focus on the case of a nonrelativistic oscillating particle having very distinct mass eigenstates. In that case, $p \sim \delta m^{2} / 2 E$, so that either $\sigma_{p} \lesssim \delta m^{2} / 2 E$ or $p \lesssim \sigma_{p}$. Under these conditions, the quantum theory of measurement says that interference between the different mass eigenstates vanishes. Once the oscillation terms have been averaged to zero, the Blasone-Vitiello formulas do not differ anymore from the standard oscillation formula (12). Therefore, the Blasone-Vitiello formalism does not seem to be relevant to the phenomenology of oscillations on macroscopic distances. This observation does not detract from the theoretical worth of that approach.

All the above models, whether for mesons or for neutrinos, can be grouped in four categories: external wave packet models, stationary boundary conditions models, source-propagator models and Blasone-Vitiello models. In the following sections, the connection between external wave packet models and stationary boundary condition models will be studied and the IoannisianPilaftsis and Shtanov models will be analyzed in detailm.

Finally let us mention again the interacting wave packet model recently proposed by Giunti [138] which was described at the end of section 2.5, since it bridges the gap between the intermediate wave packet model and quantum field treatments. Moreover its results are equivalent to those obtained in the external wave packet model.

\subsection{Five questions}

The contradictions between the existing quantum field derivations of the oscillation formula can be summarized into five questions:

1. Whereas the external wave packet model allows to associate intermediate 'wave packets' to the oscillating particle, it is not possible to do so in models using stationary boundary conditions. Moreover, the coherence length is finite in the former case, but infinite in the latter. Is it possible to see the oscillation formula derived in the models using stationary boundary conditions 187, 150, 151, 152, 163, 87, as a particular case of the oscillation formula derived in the models using external wave packets [145, 144, 85]?

2. Kiers, Nussinov and Weiss [182] have shown in a quantum-mechanical model that the value of the coherence length depends on the accuracy of the energy-momentum measurements at the detector. This effect has been confirmed by quantum field theory calculations

\footnotetext{
${ }^{11}$ Some of our results have been published in Ref. [60].
} 
[14, 183], but it is not clear whether oscillations survive a perfect measurement of the energy 144, 139]. Does a perfect knowledge of the energy lead to an infinite coherence length or is there decoherence anyway?

3. For a source strongly localized in space and time, Shtanov has derived an oscillation formula where each oscillating exponential $\exp \left(-i \phi_{j}\right)$ is multiplied by a prefactor depending on the mass $m_{j}$. Unless the masses $m_{j}$ are nearly degenerate, these prefactors strongly modify the standard oscillation formula given by Eqs. (12)-(14). Is this result correct?

4. Ioannisian and Pilaftsis [163] claim to have found a novel form of neutrino oscillations ('plane wave oscillations'), if the spatial spread of the source and detector is of macroscopic size. Does such behavior exist?

5. As in the quantum-mechanical treatment of section 2.3, the correlation between an oscillating particle and other particles (or recoil particles) at the source has been said to modify the oscillation length with respect to Eq. (14) and to bring about oscillations of the recoil particles [245, 247]. Is this assertion true?

In order to answer these questions, we shall use a model such that all specific models used in the articles cited above can be recovered in some limit. Moreover, to extend the usual treatment of a stable relativistic oscillating particle, our model will also allow the oscillating particle to be nonrelativistic and/or unstable and will take into account the dispersion. Answers to questions 1 to 4 can be found in section 7.3 while question 5 is treated in section 10 .

\section{The external wave packet model}

The numerous problems arising in the plane wave approach of oscillations (see section 2.4) and in the intermediate wave packet method (see section 2.5) show that the oscillating particle cannot be treated in isolation. The oscillation process must be considered globally: the oscillating states become intermediate states, not directly observed, which propagate between a source and a detector. This idea is easily implemented in quantum field theory, where intermediate states are represented by internal lines of Feynman diagrams. Quantum field theory has the advantage of providing a relativistic treatment from the start, which is required to study the mixings of relativistic and nonrelativistic particles. It also allows to describe unstable particles in a consistent way. The oscillating particle is represented by a relativistic propagator, which determines the space-time evolution and the possible decay of the particle. Boosts and the consideration of specific rest frames become pointless. Since interactions are included in the amplitude, equal or different time prescriptions are not needed anymore. Particles interacting with the oscillating particles at the source and at the detector are described by wave packets. This external wave packet model was first proposed by Sachs 238] for kaons and by Giunti, Kim, Lee and Lee [145] for neutrinos. 
As appears clearly from the review of section 3.1, there is an ongoing controversy in the literature about whether the boundary conditions (i.e. the source and the detector) should be taken as stationary or not. In other words, can the time independence of most oscillation experiments be translated into the assumption that the energy of the process is perfectly defined? Although it is true that most sources are stationary from a macroscopic point of view (for example, the flux of solar neutrinos is steady), there is no reason to think that it should be the case from a microscopic point of view. Whereas it can be reasonable to make a stationary approximation in a quantum-mechanical model where we do not have any information on the microscopic processes (for example a density matrix model as in [248]), the same approximation is very dubious in quantum field theory, which describes the interactions of individual particles. After all, perturbative quantum field theory applies to one-particle propagation processes, in which a stationary source and detector are the exception, rather than the rule. As emphasized by Cardall [85], the Sun is certainly not stationary at the atomic scale, and neither is a detector composed of bound state particles. For example, a water Cerenkov detector sees charged lepton wave packets with finite energy and time spread. The finite character of the spread is partly due to the limited coherence time of the bound state particle that has interacted with the incoming neutrino. Boundary conditions can be considered as stationary at the microscopic level when the energy uncertainty at the source or detector is smaller than the inverse propagation distance, i.e. $\sigma_{e P, D} \lesssim 1 / L$. This constraint is extremely stringent. In the example of atmospheric neutrinos, the process can be considered as stationary if $\sigma_{e P, D} \lesssim 10^{-19} \mathrm{MeV}$, which is not satisfied in current oscillation experiments.

Although it seems difficult to argue that the energy uncertainty is smaller than the mass difference, it is possible to take up another stand regarding stationary boundary conditions. In the same way as in section 2.5, it consists in arguing that interference occurs between wave packet components with the same energy, because of the time average on the transition probability. As it is unrealistic to consider in isolation an interference for a given energy, we should take care to integrate the probability (computed with stationary boundary conditions) over the wave packet energy width. At that point a question arises: is this energy width determined by the source or by the detector? There is thus information to be gained by working with non-stationary boundary conditions. Moreover, we shall show that stationary boundary conditions can be imposed in the external wave packet model by assigning zero velocities to some states, whereas other states are represented by plane waves.

In section 4.1, we describe the Jacob-sachs model which is the prototype for the external wave packet model. We then examine the diagonalization of mixed propagators in section 4.2. Next, we compute the transition amplitude of the process associated to particle oscillations in section 4.3. In section 4.4, we discuss the simplest model in which oscillations can be consistently described. Finally, we present in section 4.5 the external wave packet model in its Gaussian version. 


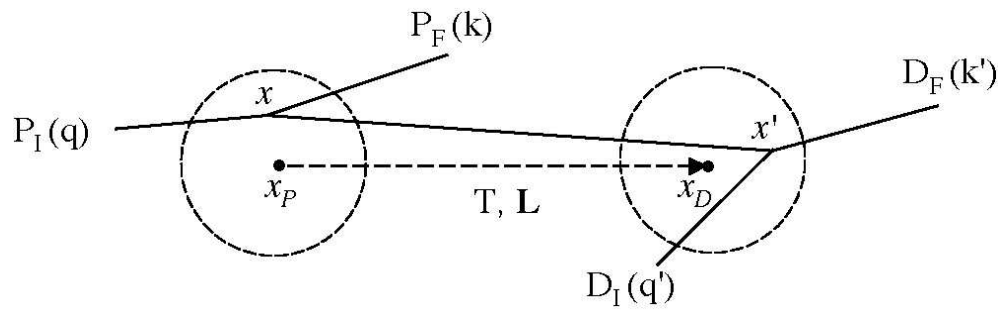

Figure 3: Propagation of a particle between a source and a detector, centered in $x_{P}$ and $x_{D}$.

\subsection{The Jacob-Sachs model}

The first version of the external wave packet model was developed by Jacob and Sachs [166] to describe the propagation of a non-oscillating unstable particle.

\subsubsection{The process}

The propagating process of a particle between a source and a detector (extended in space-time and indicated by dotted circles) is symbolized by Fig. 3 .

The arrows indicate the momentum flow. $P_{I}$ represents the set of incoming particles, of total momentum $q$, arriving in the production region (or source), which is centered around the point $\left(t_{P}, \mathbf{x}_{P}\right) . P_{F}$ represents the set of outgoing particles, of total momentum $k$, coming from the production region, with the exception of the intermediate particle whose propagation is studied. $D_{I}, D_{F}$ and $\left(t_{D}, \mathbf{x}_{D}\right)$ are defined similarly, but apply to the detection process. If the intermediate particle decays, $D_{I}\left(q^{\prime}\right)$ can be seen as an outgoing state. The interaction points at production and detection are noted $x$ and $x^{\prime}$, respectively.

The external particles are assumed to be stable, whereas the intermediate particle is allowed to be unstable. Although it is possible to describe external unstable particles by considering a more global process where all unstable particles are considered as intermediate states, the technical difficulties involved in computing the corresponding amplitudes are enormous. All computations will be carried out for a scalar propagating particle since it can be argued (see section 4.3) that the spin has no impact on the characteristics of oscillations.

With the aim of localizing the production region around $\left(t_{P}, \mathbf{x}_{P}\right)$, the in- and outgoing particles at point $x$ are represented by wave packets which overlap (in configuration space) around $\left(t_{P}, \mathbf{x}_{P}\right)$. As the energy-momenta are often measured, the wave packets are supposed to be well-localized in momentum space, around their average momentum. The detection region is localized in the same way. Note that there is no difficulty in replacing certain wave packets by bound states or by plane waves, if needed. For example, it is practical to use plane waves if an emitted particle is not observed [150].

If the dotted circles are removed, the above picture can be interpreted as a Feynman diagram. The internal line represents either a particle or an antiparticle. The experimental conditions are chosen such that a quasi-real particle propagates on a macroscopic distance, 
transferring positive energy from $x$ to $x^{\prime}$. The energy-momenta of the initial and final states are such that the S-matrix element, corresponding to the above process, is evaluated at the singularity of the propagator of the intermediate particle. If $x^{\prime 0}-x^{0}$ is a macroscopic time, it will be seen that the intermediate particle (positive energy component) contributes to the propagation, but not the intermediate antiparticle (negative energy component).

\subsubsection{Wave packets}

A wave packet representing a given state $|\psi\rangle$ of mass $m$ can be expressed in momentum space [226] as

$$
\left|\psi>=\int[d \mathbf{k}] \psi(\mathbf{k})\right| \mathbf{k}>,
$$

where $\psi(\mathbf{k})$ is the wave function in momentum space taken at time $t=0$, and $\mid \mathbf{k}>$ belongs to the basis of one-particle states of momentum $\mathbf{k}$, also taken at time $t=0$. In the interaction picture, the time-dependence of the basis and of the wave function cancel each other so that the state $\mid \psi>$ is independent of time. That is why we may take $\psi(\mathbf{k})$ and $\mid \mathbf{k}>$ at time $t=0$. The following notation is used

$$
[d \mathbf{k}]=\frac{d \mathbf{k}}{(2 \pi)^{3}} \frac{1}{\sqrt{2 E(\mathbf{k})}},
$$

where $E(\mathbf{k})=\sqrt{\mathbf{k}^{2}+m^{2}}$. The normalization of free states is chosen as follows 226]:

$$
<\mathbf{k} \mid \mathbf{p}>=2 E(\mathbf{k})(2 \pi)^{3} \delta^{(3)}(\mathbf{p}-\mathbf{k}),
$$

so that

$$
<\psi \mid \psi>=1 \quad \text { if } \quad \int \frac{d \mathbf{k}}{(2 \pi)^{3}}|\psi(\mathbf{k})|^{2}=1 .
$$

In configuration space, the wave function reads

$$
\widetilde{\psi}(\mathbf{x}, t)=\int \frac{d \mathbf{k}}{(2 \pi)^{3}} \psi(\mathbf{k}) e^{-i E(\mathbf{k}) t+i \mathbf{k} \cdot \mathbf{x}} .
$$

It is here necessary to use the time-dependent wave function, i.e. $\psi(\mathbf{k}) \exp (-i E(\mathbf{k}) t)$, since the wave function is considered alone.

If the wave packet represents a particle with an approximately known momentum $\mathbf{K}$, the wave function $\psi(\mathbf{k})$ is sharply peaked at $\mathbf{k}=\mathbf{K}$. The configuration space wave function $\widetilde{\psi}(\mathbf{x}, t=0)$ has a maximum at the point $\mathbf{x}=\mathbf{0}$ if $\psi\left(\mathbf{K}+\mathbf{k}^{\prime}\right)=\psi\left(\mathbf{K}-\mathbf{k}^{\prime}\right)$. On this assumption, the wave function will be noted $\psi(\mathbf{k}, \mathbf{K})$.

Wave packets centered in $\mathbf{x}_{0}$ at time $t_{0}$ are built with the help of the space-time translation operator $\exp \left(i \hat{P} \cdot x_{0}\right)$, where $x_{0}=\left(t_{0}, \mathbf{x}_{0}\right)$. If a wave packet in momentum space is given by

$$
\Psi\left(\mathbf{k}, \mathbf{K}, \mathbf{x}_{0}, t_{0}\right)=\psi(\mathbf{k}, \mathbf{K}) e^{i E(\mathbf{k}) t_{0}-i \mathbf{k} \cdot \mathbf{x}_{0}},
$$

the corresponding wave packet in configuration space will be peaked at the point $\mathbf{x}_{0}$ at time $t_{0}$ :

$$
\widetilde{\Psi}\left(\mathbf{x}, t, \mathbf{K}, \mathbf{x}_{0}, t_{0}\right)=\int \frac{d \mathbf{k}}{(2 \pi)^{3}} \psi(\mathbf{k}, \mathbf{K}) e^{-i E(\mathbf{k})\left(t-t_{0}\right)+i \mathbf{k} \cdot\left(\mathbf{x}-\mathbf{x}_{0}\right)} .
$$


Without loss of generality, let us choose to work with only one particle in $P_{I}(q)$, in $P_{F}(k)$, in $D_{I}\left(q^{\prime}\right)$ and in $D_{F}\left(k^{\prime}\right)$. The extension to a larger number of initial and final particles is straightforward and would only complicate the notation. The wave packets are built such that those involved in the production are centered at $\mathbf{x}_{P}$ at time $t_{P}$, whereas those involved in the detection are centered at $\mathbf{x}_{D}$ at time $t_{D}$. They are noted 145, 144

$$
\begin{aligned}
\mid P_{I}> & =\int[d \mathbf{q}] \Psi_{P_{I}}\left(\mathbf{q}, \mathbf{Q}, \mathbf{x}_{P}, t_{P}\right) \mid P_{I}(\mathbf{q})> \\
\mid P_{F}> & =\int[d \mathbf{k}] \Psi_{P_{F}}\left(\mathbf{k}, \mathbf{K}, \mathbf{x}_{P}, t_{P}\right) \mid P_{F}(\mathbf{k})> \\
\mid D_{I}> & =\int\left[d \mathbf{q}^{\prime}\right] \Psi_{D_{I}}\left(\mathbf{q}^{\prime}, \mathbf{Q}^{\prime}, \mathbf{x}_{D}, t_{D}\right) \mid D_{I}\left(\mathbf{q}^{\prime}\right)> \\
\mid D_{F}> & =\int\left[d \mathbf{k}^{\prime}\right] \Psi_{D_{F}}\left(\mathbf{k}^{\prime}, \mathbf{K}^{\prime}, \mathbf{x}_{D}, t_{D}\right) \mid D_{F}\left(\mathbf{k}^{\prime}\right)>
\end{aligned}
$$

Gaussian wave packets are discussed in section 4.5.1.

\subsubsection{The propagation amplitude}

The general formula of the connected amplitude corresponding to Fig. 3 is given by

$$
\mathcal{A}=<P_{F}, D_{F}\left|\hat{T}\left(\exp \left(-i \int d^{4} x \mathcal{H}_{I}\right)\right)-\mathbf{1}\right| P_{I}, D_{I}>
$$

where $\mathcal{H}_{I}$ is the interaction Lagrangian for the intermediate particle and $\hat{T}$ is the time ordering operator. Let $g$ be the coupling constant in $\mathcal{H}_{I}$. Expanding the amplitude to order $g^{2}$, and inserting the wave packets expressions, we obtain

$$
\mathcal{A}=\int[d \mathbf{q}] \Psi_{P_{I}} \int[d \mathbf{k}] \Psi_{P_{F}}^{*} \int\left[d \mathbf{q}^{\prime}\right] \Psi_{D_{I}} \int\left[d \mathbf{k}^{\prime}\right] \Psi_{D_{F}}^{*} \mathcal{A}_{\text {planewave }}\left(q, k, q^{\prime}, k^{\prime}\right)
$$

with

$$
\mathcal{A}_{\text {planewave }}\left(q, k, q^{\prime}, k^{\prime}\right)=\int d^{4} x M_{P}(q, k) e^{-i(q-k) \cdot x} \int d^{4} x^{\prime} M_{D}\left(q^{\prime}, k^{\prime}\right) e^{-i\left(q^{\prime}-k^{\prime}\right) \cdot x^{\prime}} G\left(x^{\prime}-x\right)
$$

where $M_{P}(q, k)$ and $M_{D}\left(q^{\prime}, k^{\prime}\right)$ are the interaction amplitudes at production and detection. The propagator $G\left(x^{\prime}-x\right)$ has been obtained by field contraction and reads

$$
G\left(x^{\prime}-x\right)=\int \frac{d^{4} p}{(2 \pi)^{4}} e^{-i p \cdot\left(x^{\prime}-x\right)} G\left(p^{2}\right)
$$

where $G\left(p^{2}\right)=i\left(p^{2}-M_{0}^{2}+i \epsilon\right)^{-1}$ is the free propagator in momentum space and $M_{0}$ is the bare mass of the propagating particle. It is assumed that renormalization has been carried out so that $M_{0}$ can be replaced by the physical mass $m$. The external particles are on shell:

$$
q^{0}=E_{P_{I}}(\mathbf{q})=\sqrt{\mathbf{q}^{2}+m_{P_{I}}^{2}}
$$

and so on. 
We have supposed that the particle $\left(p^{0}>0\right)$ propagates from $x$ to $x^{\prime}$ and the antiparticle $\left(p^{0}<0\right)$ from $x^{\prime}$ to $x$. If interactions at the source and at the detector are such that the particle propagates from $x^{\prime}$ to $x$ and the antiparticle from $x$ to $x^{\prime}$, the contraction of the fields would have yielded the propagator

$$
\bar{G}\left(x^{\prime}-x\right)=\int \frac{d^{4} p}{(2 \pi)^{4}} e^{i p \cdot\left(x^{\prime}-x\right)} G\left(p^{2}\right) .
$$

In that case, the different phase sign would select the antiparticle pole in the subsequent contour integral.

If the propagating particle is unstable, the complete propagator should replace the free propagator in the amplitude. For this purpose, the amplitude is expanded to all orders and one sums over all diagrams corresponding to one-particle-irreducible insertions (1PI self-energy) in the propagator [78, 226]. This sum is a geometrical series in the 1PI self-energy $-i \Pi\left(p^{2}\right)$ and yields the same formula as above, except that $G\left(p^{2}\right)$ represents now the complete propagator:

$$
G\left(p^{2}\right)=\frac{i}{p^{2}-M_{0}^{2}-\Pi\left(p^{2}\right)+i \epsilon} .
$$

It is convenient to proceed to a change of variables in Eq. (23):

$$
x \rightarrow x+x_{P} \quad \text { and } \quad x^{\prime} \rightarrow x^{\prime}+x_{D},
$$

where $x_{P}=\left(t_{P}, \mathbf{x}_{P}\right)$ and $x_{D}=\left(t_{D}, \mathbf{x}_{D}\right)$. The amplitude (23) becomes

$$
\mathcal{A}=\int \frac{d^{4} p}{(2 \pi)^{4}} \psi\left(p^{0}, \mathbf{p}\right) G\left(p^{2}\right) e^{-i p \cdot\left(x_{D}-x_{P}\right)}
$$

where the overlap function $\psi\left(p^{0}, \mathbf{p}\right)$ represents the overlap of the incoming and outgoing wave packets, both at the source and at the detector. It is defined by

$$
\begin{aligned}
& \psi\left(p^{0}, \mathbf{p}\right)=\int d^{4} x e^{i p x} \int d^{4} x^{\prime} e^{-i p x^{\prime}} \int[d \mathbf{q}] \psi_{P_{\text {in }}}(\mathbf{q}, \mathbf{Q}) e^{-i q x} \int[d \mathbf{k}] \psi_{P_{\text {out }}}^{*}(\mathbf{k}, \mathbf{K}) e^{i k x} \\
& \times \int\left[d \mathbf{q}^{\prime}\right] \psi_{D_{\text {in }}}\left(\mathbf{q}^{\prime}, \mathbf{Q}^{\prime}\right) e^{-i q^{\prime} x^{\prime}} \int\left[d \mathbf{k}^{\prime}\right] \psi_{D_{\text {out }}}^{*}\left(\mathbf{k}^{\prime}, \mathbf{K}^{\prime}\right) e^{i k^{\prime} x^{\prime}} M_{P}(q, k) M_{D}\left(q^{\prime}, k^{\prime}\right) .
\end{aligned}
$$

All external particles are on-shell. Note that the overlap function is independent of $x_{P}$ and $x_{D}$. The integrals over $x$ and $x^{\prime}$ in Eq. (28) yield delta functions, which impose energy-momentum conservation at the source and the detector. Remark also that the overlap function depends on the directions of incoming and outgoing momenta.

Most computations in field theory apply to microscopic processes, where in- and outgoing states can be approximated by plane waves. In that case, the wave functions $\psi_{P_{I}}, \psi_{P_{F}}, \psi_{D_{I}}$ and $\psi_{D_{F}}$ become delta functions, so that the overlap function $\psi\left(p^{0}, \mathbf{p}\right)$ is proportional to

$$
\psi\left(p^{0}, \mathbf{p}\right) \sim \delta^{(4)}\left(K+K^{\prime}-Q-Q^{\prime}\right) \delta^{(4)}(p-Q+K) M_{P}(Q, K) M_{D}\left(Q^{\prime}, K^{\prime}\right),
$$

where $Q=\left(\sqrt{\mathbf{Q}^{2}+m_{P_{I}}^{2}}, \mathbf{Q}\right)$ and so on. The momentum integral in Eq. (27) is then easy to perform and yields

$$
\mathcal{A} \sim \delta^{(4)}\left(K+K^{\prime}-Q-Q^{\prime}\right) G\left((Q-K)^{2}\right) e^{-i(Q-K) \cdot\left(x_{D}-x_{P}\right)} .
$$


This expression cannot lead to oscillations in a superposition of several amplitudes, since the phase of the exponential does not depend on the mass of the intermediate state. Thus, oscillations vanish in the plane wave limit.

In the case of an amplitude corresponding to a macroscopic process, such as the propagation on a macroscopic distance, the overlap function is not a delta function so that the momentum integral in Eq. (27) is not trivial (see section 4.4 for a simple exact model). The main contribution comes from the pole associated to the propagating particle, so that the phase of the exponential will depend on the mass.

\subsection{Mixed propagators}

In section 2.1, mixing was defined in quantum mechanics as the result of the noncoincidence of the flavor basis and the mass basis. The definition of mixing is similar in field theory, except that it applies to fields, not to physical states. This difference allows to bypass the problems arising in the definition of flavor and mass bases.

The complete Lagrangian is split in a propagation Lagrangian $L_{\text {propag }}$ and an interaction Lagrangian $L_{\text {int }}$. These two subsets of the Lagrangian can be distinguished if there is a flavor transformation which is a symmetry of $L_{\text {int }}$ but not of $L_{\text {propag. }}$. Particle mixing occurs if the propagator built from $L_{\text {propag }}$, and representing the creation of a particle of flavor $\alpha$ at point $x$ and the annihilation of a particle of flavor $\beta$ at point $x^{\prime}$, is not diagonal, i.e. not zero for $\alpha \neq \beta$. The Lagrangian $L_{\text {propag }}$ always includes the kinetic and the mass terms. If the particle is unstable, $L_{\text {propag }}$ also contains the interaction causing the decay.

In the simplest case, illustrated by the light neutrinos $\nu_{e}, \nu_{\mu}$ and $\nu_{\tau}$, the propagating particle is stable and the form of its fundamental Lagrangian is known (although the mass matrix might have its origin in an effective Lagrangian). $L_{\text {propag }}$ contains the mass matrix, generated by Yukawa interactions, whereas $L_{\text {int }}$ includes the weak interactions. In the flavor basis (called the gauge eigenstates basis for neutrinos), the mixed propagator is nondiagonal. It is well-known [208, 64] that $L_{\text {propag }}$ can be diagonalized by a unitary transformation on the fields [2]:

$$
\nu_{\alpha}=\sum_{j} V_{\alpha j}^{\dagger} \nu_{j}
$$

where Greek and Latin indices refer to the flavor and to the mass basis, respectively. It could however turn out that only a subset of $V$ is used to describe the mixing in an oscillation experiment. One reason could be that some of the mixed states are too heavy to be produced

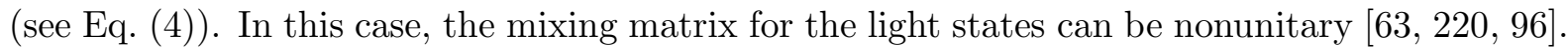
Another reason could be that some of the mixed states are sterile, i.e. they only interact through mass mixing. In that case, the mixing matrix for the non-sterile states is rectangular and does not satisfy unitarity [240].

The propagator is defined by the time-ordered two-point function:

$$
G_{\beta \alpha}\left(x^{\prime}-x\right)=<0\left|T\left(\nu_{\beta}\left(x^{\prime}\right) \nu_{\alpha}^{*}(x)\right)\right| 0>.
$$

\footnotetext{
${ }^{12}$ The matrix $V$ corresponds to the matrix $U^{t}$ of section 2.1.
} 
Since Wick's theorem applies to fields in the mass basis, we must substitute Eq. (29) in this equation. In the scalar case, field contraction yields

$$
G_{\beta \alpha}\left(x^{\prime}-x\right)=\sum_{j} V_{\beta j}^{\dagger} G_{D, j j}\left(x^{\prime}-x\right) V_{j \alpha},
$$

where $G_{D, j j}\left(x^{\prime}-x\right)$ is the free propagator (with $D$ for 'diagonal') of a scalar particle of mass $m_{j}$ :

$$
G_{D, j j}\left(x^{\prime}-x\right)=\int \frac{d^{4} p}{(2 \pi)^{4}} e^{-i p\left(x^{\prime}-x\right)} \frac{i}{p^{2}-m_{j}^{2}+i \epsilon} .
$$

The derivation of the mixed propagator for a spin one-half particle is similar.

The treatment of unstable particles is more involved. The complete Lagrangian is again split in $L_{i n t}$ and $L_{\text {propag }}$, but the interactions causing the decay should be included in $L_{\text {propag }}$. The free propagator is then replaced by the complete propagator, obtained by an infinite sum on the self-energy $-i \Pi\left(p^{2}\right)[49]$ :

$$
i G^{-1}\left(p^{2}\right)=p^{2} \mathbf{1}-M_{0}^{2}-\Pi\left(p^{2}\right),
$$

in an obvious matrix notation. The propagation eigenstates are characterized by the complex poles of the complete propagator $z_{j}=m_{j}^{2}-i m_{j} \Gamma_{j}$ or, equivalently, by the zeros of the inverse propagator. It is always possible to diagonalize the propagator if its eigenvalues are distinct:

$$
G\left(p^{2}\right)=V^{-1}\left(p^{2}\right) G_{D}\left(p^{2}\right) V\left(p^{2}\right)
$$

where $G_{D}\left(p^{2}\right)$ is the diagonal matrix the elements of which are given by

$$
G_{D, j j}\left(p^{2}\right)=\frac{i}{p^{2}-m_{j}^{2}+i m_{j} \Gamma_{j}} .
$$

The matrix $V$ is not necessarily unitary, since the self-energy matrix for unstable particles is usually not normal (see section 2.1). Moreover, it depends on the energy. If the mass eigenstates

are nearly degenerate, the self-energy can be approximated by its value at $p^{2}=m^{2}$ and the matrix $V$ becomes constant. In section 8.1, we show that the energy dependence of the selfenergy generates corrections to the amplitude in inverse powers of the propagation distance $L$. These corrections are due to production thresholds of multi-particles states [241, 166, 78].

\subsection{The flavor-mixing amplitude}

In order to derive the flavor-mixing amplitude, the Jacob-Sachs model of section 4.1 has to be modified to take into account the different flavors. The process corresponding to the production at the source of a particle of flavor $\alpha$, and the detection of a particle of flavor $\beta$ at a detector placed at a macroscopic distance from the source, can still be symbolized by the Fig. 3 of section 4.1.1, except that the intermediate particle should be considered as a superposition of different mass eigenstates. The initial flavor $\alpha$ is tagged, for example, by the outgoing state $P_{F}(k)$, whereas the final flavor $\beta$ can be tagged by the outgoing state $D_{F}\left(k^{\prime}\right)$. If it is impossible to 
identify the flavor at the detector (ex: $K^{0}, \bar{K}^{0} \rightarrow \pi^{+} \pi^{-}$), one should sum over the different flavors.

If the energy dependence of the matrix $V$ diagonalizing the propagator can be neglected (see Eq. (32)), the amplitude corresponding to the global process can be expressed as a linear combination of amplitudes $\mathcal{A}_{j}$ corresponding to the propagation of different mass eigenstates:

$$
\mathcal{A}(\alpha \rightarrow \beta, T, \mathbf{L})=\sum_{j} V_{\beta j}^{-1} \mathcal{A}_{j} V_{j \alpha}
$$

where the average propagation time $T$ is defined by $T=x_{D}^{0}-x_{P}^{0}$ and the average propagation distance by $\mathbf{L}=\mathbf{x}_{D}-\mathbf{x}_{P}$. The partial amplitude $\mathcal{A}_{j}$ has the same form as the propagation amplitude of an isolated particle, given in Eq. (27):

$$
\mathcal{A}_{j}=\int \frac{d^{4} p}{(2 \pi)^{4}} \psi\left(p^{0}, \mathbf{p}\right) G_{D, j j}\left(p^{2}\right) e^{-i p^{0} T+i \mathbf{p} \cdot \mathbf{L}} .
$$

The overlap function $\psi\left(p^{0}, \mathbf{p}\right)$ is defined by Eq. (28) and the propagator for the $j$ th mass eigenstate by Eq. (33).

As noted in section 4.1.1, it will be enough for our purpose to work with a scalar oscillating particle. When the spin is taken into account, three cases must be distinguished:

1. If the particle is relativistic and the interactions at the source and detector are chiral, only one helicity eigenstate contributes to the propagation. The spin structure can then be factorized and the computation can proceed with a scalar propagator. The two mentioned requirements are satisfied for light neutrino oscillations, since the energy threshold of the detectors is much higher than the light neutrino mass scale, and because of the chirality of the Standard Model flavor-changing interactions.

2. If the particle is nonrelativistic, the spin structure is approximately equal for the different helicities provided that the mass eigenstates are nearly degenerate: $m_{i} \cong m_{j} \equiv m$. The helicity factors can then be factorized from the sum over the mass eigenstates:

$$
\mathcal{P}_{\alpha \rightarrow \beta}^{\text {spin }}(T, \mathbf{L}) \sim\left(\sum_{s}|H(s, m)|^{2}\right)\left|\mathcal{A}_{\alpha \rightarrow \beta}^{\text {scalar }}(T, \mathbf{L})\right|^{2}
$$

where $H(s, m)$ includes all helicity dependent factors (with the index $s$ referring to the spins of the external particles). However the source and detector contributions to $\sum_{s}|H(s, m)|^{2}$ cannot be disentangled if the particle is not relativistic, so that the whole process cannot be factorized into a product of source/propagation/detection probabilities (this point was emphasized Refs. 145, 87]). As the mass eigenstates are nearly degenerate, it seems natural to define the oscillation probability as the modification introduced by the mass difference:

$$
\mathcal{P}_{\alpha \rightarrow \beta}(T, \mathbf{L})=\frac{\mathcal{P}_{\alpha \rightarrow \beta}^{\text {spin }}(T, \mathbf{L})}{\left.\mathcal{P}_{\alpha \rightarrow \beta}^{\text {spin }}(T, \mathbf{L})\right|_{m_{i}=m_{j}}},
$$

so that the calculation of the oscillation can be done as if the oscillating particle were scalar. The discussion is very similar for a relativistic particle with nonchiral interactions. 
3. If the particle is nonrelativistic and the mass eigenstates have very different masses, each specific process has to be computed separately, with the influences of the amplitudes of production and detection carefully taken into account for each mass eigenstate. This situation is not really relevant to oscillations, but rather to incoherent mixing, since oscillations are averaged to zero in such experimental conditions. In other words, the contributions of the Feynman diagrams corresponding to the different mass eigenstates are summed in the probability, not in the amplitude.

In this report, we always assume the condition $\delta m_{i j}^{2} \ll E^{2}$, under which oscillations are observable, so that the third case is not considered. Some explicit examples of the third case have been given by Giunti, Kim and Lee [142], and by Kiers and Weiss [183]. The conditions of factorization of the transition probability have been discussed by Cardall and Chung [87].

\subsection{The simplest consistent model}

A simple model, in which the flavor-changing amplitude (Eqs. (34)-(35)) can be computed exactly, has been proposed by Kobzarev, Martemyanov, Okun and Shchepkin [187]. In this model, a charged lepton, represented by a plane wave, collides with an infinitely heavy nucleus, situated in $\mathbf{x}_{P}$. The neutrino produced at that point has a definite energy equal to the energy of the incident lepton. At point $\mathbf{x}_{D}$, the neutrino collides with a second infinitely heavy nucleus and, as a result of this collision, a charged lepton is emitted with an energy equal to the neutrino energy.

Let us see what these assumptions mean in the wave packet notation of section 4.1.2. There are two ingoing states at the source, the lepton and the nucleus. The lepton is represented by a plane wave, so that

$$
\psi_{P_{I, l e p t}}\left(\mathbf{q}_{l}, \mathbf{Q}_{l}\right) \sim \delta^{(3)}\left(\mathbf{q}_{l}-\mathbf{Q}_{l}\right) .
$$

The uncertainty on the momentum of the nucleus is infinite, so that the ingoing and outgoing momentum wave functions are constant:

$$
\psi_{P_{I, n u c l}}\left(\mathbf{q}_{n}, \mathbf{Q}_{n}\right) \sim \text { const } \quad \text { and } \quad \psi_{P_{F, n u c l}}\left(\mathbf{k}_{n}, \mathbf{K}_{n}\right) \sim \text { const }
$$

The situation is similar at the detector, except that there are one ingoing and two outgoing states.

The overlap function (28) is easily computed:

$$
\psi\left(p^{0}, \mathbf{p}\right) \sim \delta\left(p^{0}-E_{\text {in }}\right) \delta\left(p^{0}-E_{\text {out }}\right),
$$

where $E_{\text {in }}$ is the energy of the incoming lepton at the source and $E_{\text {out }}$ is the energy of the outgoing lepton at the detector. The partial amplitude (35) becomes

$$
\mathcal{A}_{j} \sim \delta\left(E_{\text {in }}-E_{\text {out }}\right) \int d^{3} p G_{D, j j}\left(E_{\text {in }}, \mathbf{p}\right) e^{i \mathbf{p} \cdot \mathbf{L}},
$$


where $\mathbf{L}=\mathbf{x}_{D}-\mathbf{x}_{P}$. The Fourier transform of the propagator with respect to its momentum can be computed by contour integration and yields

$$
\mathcal{A}_{j} \sim \frac{1}{L} \delta\left(E_{\text {in }}-E_{\text {out }}\right) e^{i p_{j} L}
$$

where $p_{j}=\sqrt{E_{\text {in }}^{2}-m_{j}^{2}}$ and $L=|\mathbf{L}|$.

The transition probability between an initial state of flavor $\alpha$ and a final state of flavor $\beta$ is given by the squared modulus of the amplitude (34) with $\mathcal{A}_{j}$ given by Eq. (37). Oscillations between mass eigenstates $m_{i}$ and $m_{j}$ arise from interference terms $\mathcal{A}_{i} \mathcal{A}_{j}^{*} \sim e^{i\left(p_{i}-p_{j}\right) L}$. Thus, the $i j$-interference term oscillates with a frequency equal to

$$
\left(p_{i}-p_{j}\right) L \cong \frac{\delta m_{i j}^{2}}{2 p_{m}} L,
$$

where $\delta m_{i j}^{2} \equiv m_{i}^{2}-m_{j}^{2}$ and $p_{m}=\sqrt{E_{i n}^{2}-m^{2}}$, with $m$ the mass in the degenerate limit. The phase (38) is equal to the standard oscillation phase (14) calculated in the quantum-mechanical plane wave treatment.

This very simple model has the advantage of being consistent, unlike the plane wave approach in quantum mechanics. It could be recommended as a pedagogical tool. It is however not sufficient for a thorough study of the oscillation phenomenon. First, it cannot describe unstable particles, since they cannot decay into an infinitely heavy state. Moreover, the approximation of plane waves and stationary states is too strong to allow the study of the observability conditions of oscillations. Another drawback of this model is that the amplitude is independent of the direction of $\mathbf{L}$. Last, a spatial localization of the source or the detector more precise than the Compton wave length of the lightest external particle is unphysical [165].

\subsection{The overlap function for Gaussian wave packets}

In most cases, it is not possible to compute exactly the flavor-changing amplitude (34). The technical difficulty lies in the energy-momentum integration in Eq. (35). The two exceptions are, on the one hand, the Kobzarev et al. model of section 4.4 and, and the other hand, the limit in which all the external wave packets are plane waves (in which case oscillations vanish as shown in section 4.1.3). To proceed further, we have to be more specific about the shape of the overlap function (28). In order to answer the questions on the coherence length, it is useful to work with an overlap function which depends explicitly on the energy and 3-momentum uncertainties at the source and at the detector. This can be done by approximating the in- and outgoing particles with Gaussian wave packets, as first proposed by Giunti, Kim, Lee and Lee [145, 144]. The general case of arbitrary wave packets can then be analyzed as a superposition of Gaussian wave packets. Note that the Gaussian approximation is used by most authors since it allows analytical integrations. 


\subsubsection{Gaussian wave packets}

General wave packets were defined in section 4.1.2. The wave function of Gaussian wave packet can be written at time $t=0$ in momentum space as

$$
\psi_{\chi}\left(\mathbf{p}, \mathbf{p}_{\chi}\right)=\left(\frac{2 \pi}{\sigma_{p \chi}^{2}}\right)^{3 / 4} \exp \left(-\frac{\left(\mathbf{p}-\mathbf{p}_{\chi}\right)^{2}}{4 \sigma_{p \chi}^{2}}\right) .
$$

We chose to set the initial phase to zero. This wave packet is thus centered in $\mathbf{x}=\mathbf{0}$ at time $t=0$ (see Eq. (22)). Recall that the normalization is given by Eq. (20). The width $\sigma_{p \chi}$ is the momentum uncertainty of the wave packet, as can be checked by computing the variance of the operator $\hat{p}^{a}$ :

$$
<\left(\delta p^{a}\right)^{2}>_{\chi}=<\chi\left|\left(\hat{p}^{a}-\bar{p}_{\chi}^{a}\right)^{2}\right| \chi>=\sigma_{p \chi}^{2},
$$

with the average momentum given by

$$
\bar{p}_{\chi}^{a}=<\chi\left|\hat{p}^{a}\right| \chi>=p_{\chi}^{a}
$$

It is also useful to define a width $\sigma_{x \chi}$ in configuration space by $\sigma_{p \chi} \sigma_{x \chi}=1 / 2$.

The wave function is given in configuration space by Eq. (21). This integral is Gaussian if the energy is expanded to second order around the average momentum $\mathbf{p}_{\chi}$ :

$$
\sqrt{\mathbf{p}^{2}+m_{\chi}^{2}} \cong E_{\chi}+\mathbf{v}_{\chi} \cdot\left(\mathbf{p}-\mathbf{p}_{\chi}\right)+\frac{1}{2 E_{\chi}}\left(\left(\mathbf{p}-\mathbf{p}_{\chi}\right)^{2}-\left(\mathbf{v}_{\chi} \cdot\left(\mathbf{p}-\mathbf{p}_{\chi}\right)\right)^{2}\right)
$$

where $E_{\chi}=\sqrt{\mathbf{p}_{\chi}^{2}+m_{\chi}^{2}}$ and $\mathbf{v}_{\chi}=\mathbf{p}_{\chi} / E_{\chi}$. The wave packet is thus given in configuration space by

$$
\tilde{\psi}_{\chi}(\mathbf{x}, t)=\frac{\left(2 \pi \sigma_{x \chi}^{2}\right)^{-3 / 4}}{\sqrt{\operatorname{det} \Sigma}} \exp \left(-E_{\chi} t+i \mathbf{p}_{\chi} \cdot \mathbf{x}-\frac{\left(\mathbf{x}-\mathbf{v}_{\chi} t\right) \Sigma^{-1}\left(\mathbf{x}-\mathbf{v}_{\chi} t\right)}{4 \sigma_{x \chi}^{2}}\right),
$$

where the matrix notation is implicit and with the matrix $\Sigma$ defined by

$$
\Sigma^{i j}=\delta^{i j}+\left(\delta^{i j}-v_{\chi}^{i} v_{\chi}^{j}\right) \frac{2 i t \sigma_{p}^{2}}{E_{\chi}} .
$$

The spatial uncertainty of the wave packet can be computed in configuration space with Eq. (41):

$$
<\left(\delta x^{a}\right)^{2}>_{\chi}=<\chi\left|\left(\hat{x}^{a}-\bar{x}_{\chi}^{a}\right)^{2}\right| \chi>=\sigma_{x \chi}^{2}\left[\left(\operatorname{Re} \Sigma^{-1}\right)^{-1}\right]^{a a},
$$

where the average position is given by

$$
\bar{x}_{\chi}^{a}=<\chi\left|\hat{x}^{a}\right| \chi>=v_{\chi}^{a} t .
$$

If the $z$ axis is chosen along $\mathbf{v}_{\chi}$, we obtain

$$
\begin{aligned}
& {\left[\left(\operatorname{Re} \Sigma^{-1}\right)^{-1}\right]^{x x}=\left[\left(\operatorname{Re} \Sigma^{-1}\right)^{-1}\right]^{y y}=1+\frac{4 \sigma_{p \chi}^{4} t^{2}}{E_{\chi}^{2}}} \\
& {\left[\left(\operatorname{Re} \Sigma^{-1}\right)^{-1}\right]^{z z}=1+\frac{4 m_{\chi}^{4} \sigma_{p \chi}^{4} t^{2}}{E_{\chi}^{6}} .}
\end{aligned}
$$


These equations show that the wave packet begins to spread in directions transverse to $\mathbf{v}_{\chi}$ at $t \cong$ $E_{\chi} / 2 \sigma_{p \chi}^{2}$, whereas the spreading along $\mathbf{v}_{\chi}$ begins at the later time $t \cong E_{\chi}^{3} / 2 m_{\chi}^{2} \sigma_{p \chi}^{2}$. In this report, we refer to the time-dependence of Eq. (43) as the dispersion of the wave packet. Although dispersion will be neglected in the external wave packets, we shall see that the propagation amplitude associated to the oscillating particle spreads in the same way.

In order to study the stationary limit $\mathbf{v}_{\chi} \rightarrow 0$, it is of great interest to compute the energy uncertainty of the wave packet (as proposed in Ref. [138]). The average energy $\bar{E}_{\chi}$ and the average squared energy should both be computed to order $\sigma_{p}^{2}$. In momentum space, the average energy is given by

$$
\bar{E}_{\chi}=<\chi|\hat{E}| \chi>=E_{\chi}+\left(3-\mathbf{v}_{\chi}^{2}\right) \frac{\sigma_{p}^{2}}{2 E_{\chi}} .
$$

The second term comes from the second order term in the expansion of the energy operator around the average momentum. Remarkably the average energy $\bar{E}_{\chi}$ is different from $E_{\chi}=$ $\sqrt{\mathbf{p}_{\chi}^{2}+m_{\chi}^{2}}$. The average squared energy can be computed exactly:

$$
<\chi\left|\hat{E}^{2}\right| \chi>=E_{\chi}^{2}+3 \sigma_{p}^{2}
$$

so that the squared energy uncertainty is given to order $\sigma_{p}^{2}$ by

$$
<(\delta E)^{2}>_{\chi}=<\chi\left|\left(\hat{E}-\bar{E}_{\chi}\right)^{2}\right| \chi>=\mathbf{v}_{\chi}^{2} \sigma_{p}^{2} .
$$

In configuration space, the same results are obtained only if the dispersion (i.e. time spread) of the wave packet is taken into account. We compute to order $\sigma_{p}^{2}$ (additional terms appear at higher order) the average energy and squared energy:

$$
\begin{aligned}
<\chi|\hat{E}| \chi> & =E_{\chi}-\frac{i}{4} \operatorname{Tr}\left(\frac{\partial \Sigma}{\partial t}\right) \\
<\chi\left|\hat{E}^{2}\right| \chi> & =E_{\chi}^{2}+\mathbf{v}_{\chi}^{2} \sigma_{p}^{2}-\frac{i E_{\chi}}{2} \operatorname{Tr}\left(\frac{\partial \Sigma}{\partial t}\right) .
\end{aligned}
$$

With the help of Eq. (42), we can check that Eqs. (49) and (50) are equal to Eqs. (46) and (47), respectively (but only if dispersion is not neglected). Thus the squared energy uncertainty is the same in both representations as expected (similar computations for the momentum uncertainty show that its value is the same in momentum and configuration space even if dispersion is neglected).

Note that the energy uncertainty coincides at first order with the naive expectation:

$$
\delta E \cong \sqrt{\left(\mathbf{p}_{\chi}+\delta \mathbf{p}\right)^{2}+m^{2}}-\sqrt{\mathbf{p}_{\chi}^{2}+m^{2}} \cong \mathbf{v}_{\chi} \cdot \delta \mathbf{p} .
$$

It could be objected that the squared energy uncertainty does not vanish to order $\sigma_{p}^{4} / E_{\chi}^{2}$ in the limit $\mathbf{v}_{\chi} \rightarrow 0$. However external wave packet models are always built with non-spreading wave packets, i.e. one sets $\Sigma^{i j}=\delta^{i j}$. If this approximation is taken seriously, we can forget about the original expression in momentum space. In that case, the average energy and squared energy computed in configuration space are shifted to $E_{\chi}$ and $E_{\chi}^{2}+\mathbf{v}_{\chi}^{2} \sigma_{p}^{2}$ respectively with 
no correction of higher order in $\sigma_{p}^{2}$ (see Eqs.(49)-(50)), so that the energy uncertainty is still given by Eq. (48). Thus the energy uncertainty of non-spreading wave packets vanishes in the stationary limit $\mathbf{v}_{\chi} \rightarrow 0$ to all orders in $\sigma_{p}^{2}$.

This result is of great interest to study the stationary limit of the external wave packet model. For example, the bound state wave function used by Grimus and Stockinger [150] coincides with the wave function of a non-spreading wave packet at rest, i.e. Eq. (41) with $\mathbf{v}_{\chi}=0$ and $\Sigma=1$. Besides, plane waves can be obtained by taking $\sigma_{x \chi} \rightarrow \infty$ in Eq. (41).

\subsubsection{Gaussian overlap function}

We now compute the overlap function (28) with non-spreading external wave packets as done in Ref. [144]. The factors $M_{P}(q, k)$ and $M_{D}\left(q^{\prime}, k^{\prime}\right)$ multiplying the exponential vary slowly over the width of the wave packet and can be approximated by their value at the average momentum. They can be factorized outside the sum over the mass eigenstates since it is assumed that the neutrinos are either relativistic or nearly degenerate in mass. The wave packets $\psi_{P_{\text {in,out }}}$ and

$\psi_{D_{\text {in }, \text { out }}}$ have the form given in Eq. (39). Expanding the energy to first order around the average momenta, the momentum integrations in Eq. (28) give results similar to Eq. (41) but with $\Sigma=1$ and additional factors $\sqrt{2 E}$ coming from the integration measure. For example we obtain

$$
\int[d \mathbf{q}] \psi_{P_{i n}}(\mathbf{q}, \mathbf{Q}) e^{-i q x}=N_{P_{i n}} \exp \left(-i E_{P_{i n}}(\mathbf{Q}) t+i \mathbf{Q} \cdot \mathbf{x}-\frac{\left(\mathbf{x}-\mathbf{v}_{P_{i n}} t\right)^{2}}{4 \sigma_{x P_{i n}}^{2}}\right)
$$

where $N_{P_{\text {in }}}=\left(2 \pi \sigma_{x P_{i n}}^{2}\right)^{-3 / 4}\left(2 E_{P_{i n}}(\mathbf{Q})\right)^{-1 / 2}$ is a normalization constant.

Doing the same for the other wave packets, we can write the overlap function as

$$
\psi\left(p^{0}, \mathbf{p}\right)=N \psi_{P}\left(p^{0}, \mathbf{p}\right) \psi_{D}^{*}\left(p^{0}, \mathbf{p}\right)
$$

with

$$
\psi_{P}\left(p^{0}, \mathbf{p}\right)=\int d^{4} x \exp \left(i\left(p^{0}-E_{P}\right) t-i\left(\mathbf{p}-\mathbf{p}_{P}\right) \cdot \mathbf{x}-\frac{\mathbf{x}^{2}-2 \mathbf{v}_{P} \cdot \mathbf{x} t+\Sigma_{P} t^{2}}{4 \sigma_{x P}^{2}}\right),
$$

where $E_{P}=E_{P_{\text {in }}}-E_{P_{\text {out }}}, \mathbf{p}_{P}=\mathbf{Q}-\mathbf{K}$. The function $\psi_{D}\left(p^{0}, \mathbf{p}\right)$ is defined in the same way, with the index $P$ replaced by $D$, except for the energy-momentum which is defined so as to be positive: $E_{D}=E_{D_{\text {out }}}-E_{D_{\text {in }}}$ and $\mathbf{p}_{D}=\mathbf{K}^{\prime}-\mathbf{Q}^{\prime}$. The constant $N$ includes the normalization constants as well as the factors $M_{P, D}$ evaluated at the maxima of the wave packets.

A new width $\sigma_{p P}$ has been defined by $\sigma_{p P} \sigma_{x P}=1 / 2$, with

$$
\frac{1}{\sigma_{x P}^{2}}=\frac{1}{\sigma_{x P_{\text {in }}}^{2}}+\frac{1}{\sigma_{x P_{\text {out }}}^{2}}
$$

$\sigma_{p P}$ can be interpreted as the momentum uncertainty at the source. The width $\sigma_{x P}$ is mainly determined by the external particle with the smallest space width. This is expected since the production region depends on the overlap in space-time of the external wave packets. 
The symbol $\mathbf{v}_{P}$ is defined by

$$
\mathbf{v}_{P}=\sigma_{x P}^{2}\left(\frac{\mathbf{v}_{P_{\text {in }}}}{\sigma_{x P_{\text {in }}}^{2}}+\frac{\mathbf{v}_{P_{\text {out }}}}{\sigma_{x P_{\text {out }}}^{2}}\right) .
$$

It can be interpreted as the velocity of the production region, approximately equal to the velocity of the particle with the smallest spatial spread (unless the velocities of the different in- and outgoing particles are very different).

The symbol $\Sigma_{P}$, satisfying $0 \leq \Sigma_{P} \leq 1$, is defined by

$$
\Sigma_{P}=\sigma_{x P}^{2}\left(\frac{\mathbf{v}_{P_{\text {in }}}^{2}}{\sigma_{x P_{\text {in }}}^{2}}+\frac{\mathbf{v}_{P_{\text {out }}}^{2}}{\sigma_{x P_{\text {out }}}^{2}}\right) .
$$

Recall in all above definitions that there might be more than one in- and outgoing state in the production and detection process.

Integrating over $\mathbf{x}$ and $t$, we obtain

$$
\psi_{P}\left(p^{0}, \mathbf{p}\right)=\pi^{2} \sigma_{p P}^{-3} \sigma_{e P}^{-1} \exp \left(-f_{P}\left(p^{0}, \mathbf{p}\right)\right)
$$

with

$$
f_{P}\left(p^{0}, \mathbf{p}\right)=\frac{\left(\mathbf{p}-\mathbf{p}_{P}\right)^{2}}{4 \sigma_{p P}^{2}}+\frac{\left(p^{0}-E_{P}-\left(\mathbf{p}-\mathbf{p}_{P}\right) \cdot \mathbf{v}_{P}\right)^{2}}{4 \sigma_{e P}^{2}}
$$

where

$$
\sigma_{e P}^{2}=\sigma_{p P}^{2}\left(\Sigma_{P}-\mathbf{v}_{P}^{2}\right) \leq \sigma_{p P}^{2} .
$$

The quantity $\sigma_{e P}$ can be interpreted as the energy uncertainty at the source, or also as the inverse of the time of overlap of wave packets during the production process. Indeed, we can show that

$$
\sigma_{e P}^{2}=\sum_{\alpha<\beta} \frac{\sigma_{x P}^{2}}{4 \sigma_{x \alpha}^{2} \sigma_{x \beta}^{2}}\left(\mathbf{v}_{\alpha}-\mathbf{v}_{\beta}\right)^{2}
$$

where the sum is over all wave packets involved in the production process. This sum is dominated by the term including the two smallest wave packets in configuration space (unless their velocities are nearly equal). If $\sigma_{x 1}$ is the smallest width and $\sigma_{x 2}$ the second smallest, we obtain

$$
\sigma_{e P} \sim \frac{\left|\mathbf{v}_{1}-\mathbf{v}_{2}\right|}{\sigma_{x 2}} \sim \frac{1}{T_{P}^{\text {overlap }}}
$$

where $T_{P}^{\text {overlap }}$ is defined as the duration of the production process. Thus, $\sigma_{e P}$ can be interpreted as the energy uncertainty at the source, since it is proportional to the inverse of the time of overlap of the external wave packets at the source. The quantities $\sigma_{x D}, \sigma_{p D}, \mathbf{v}_{D}, \Sigma_{D}, \sigma_{e D}$, $T_{D}^{\text {overlap }}$ have similar definitions and properties.

Note that stationary boundary conditions are recovered by setting $\mathbf{v}_{P, D}=0$ and $\sigma_{e P, D}=0$, with $\sigma_{p P, D}$ different from zero. Besides, we shall need to know the stationary limit of $\left|\mathbf{v}_{P, D}\right| / \sigma_{e P, D}$. Since $\mathbf{v}_{P}$ is the velocity of the production region and $\sigma_{e P}^{-1}$ is the duration of the production 
process, the ratio $\left|\mathbf{v}_{P}\right| / \sigma_{e P}$ is bounded by the size $S_{P}$ of the macroscopic region of production. The argument is similar for the ratio $\left|\mathbf{v}_{D}\right| / \sigma_{e D}$. Thus we assume the following constraints:

$$
\frac{\left|\mathbf{v}_{P, D}\right|}{\sigma_{e P, D}} \lesssim S_{P, D}
$$

These bounds are very conservative, since we shall see that stationary models such as those found in Refs. 150, 163 are recovered by setting $\left|\mathbf{v}_{P, D}\right| / \sigma_{e P, D}=0$. In the example of the Grimus-Stockinger model [150], an initial stationary neutron $\left(\mathbf{v}_{P_{i n}, n}=0\right)$ decays into a stationary proton $\left(\mathbf{v}_{P_{\text {out }}, p r}=0\right)$, a 'plane-wave' electron $\left(\sigma_{x P_{\text {out }}, e l}=\infty\right)$ and the intermediate antineutrino. At detection, the antineutrino collides with a stationary electron $\left(\mathbf{v}_{D_{\text {in }}}=0\right)$ and the outgoing antineutrino and electron are represented as plane waves $\left(\sigma_{x D_{\text {out }}, \nu}=\sigma_{x D_{\text {out }}, \text { el }}=\infty\right)$. As argued at the end of section 4.5.1, all these stationary states are limiting cases of wave packets.

As the propagation distance is macroscopic, only processes satisfying global conservation of energy-momentum have a nonnegligible probability of occurring. Since our aim is not to prove this well-known fact, we impose that

$$
\mathbf{p}_{P}=\mathbf{p}_{D} \equiv \mathbf{p}_{0} \quad \text { and } \quad E_{P}=E_{D} \equiv E_{0},
$$

but we still have $\mathbf{v}_{P} \neq \mathbf{v}_{D}$. These approximations allow to do expansions around $\mathbf{p}_{0}$ and $E_{0}$. An associated velocity can be defined by $\mathbf{v}_{0}=\mathbf{p}_{0} / E_{0}$.

\section{Propagation amplitude and dispersion}

\section{$5.1 \quad$ Introduction}

In section 1, we described the external wave packet model and we applied to flavor-mixing transitions. All the information relevant to the source and detector was included in what we called the overlap function (Eq. (28)). We computed this overlap function, on the one hand, in the pedagogical case of infinitely heavy source and detector (Eq. (36)) and, on the other hand, in the more general case of Gaussian external wave packets (Eqs. (53)-(55)). It is now possible to evaluate the transition amplitude.

Although the flavor-mixing amplitude, as defined in Eqs. (34) -(35), is the common starting point of most field-theoretical models found in the literature, the obtained oscillation formulas do not always agree. In section 3, several conflicting results were mentioned, namely stationary states versus non-stationary states, the possible existence of plane wave oscillations and of mass prefactors. Actually, it will be seen in sections 6 and 7 that these apparent contradictions vanish if we proceed to a careful evaluation of the integrals present in Eq. (35). In the present section, the possible methods of evaluating the amplitude (35) are compared. We shall see that the choice of the integration method depends on the propagation distance, so that the distance range can be divided in three regimes. We then evaluate the amplitude in the three scenarios. 


\subsection{Pole integration and wave packet correspondence}

Since the experimental conditions are such that the propagating particle is on-shell, the main contribution to the transition amplitude (35) comes from the pole of the propagator. Although integrals on $p^{0}$ and $\mathbf{p}$ can, in principle, be done in any order, it is practical to integrate first on the energy, i.e. to do the pole integration on the energy, in order to make possible an interpretation of the amplitude in terms of propagating wave packets. In section 6.4, we shall discuss another method of integration (for stable oscillating particles) that has the advantages of being shorter and of clearing up the meaning of the stationary limit. However, this last method neither lends itself to a wave packet picture nor can be easily extended to unstable oscillating particles.

The crucial step in the pole integration is the choice of an appropriate contour in the complex plane. The contour should be carefully chosen as the analytic continuation of most overlap functions diverges at infinity in the complex plane. The integration on the energy can be performed with the help of the Jacob-Sachs theorem [166]. This theorem is based on the assumption that the energy spectrum of all incident particles is limited to a finite range. Thus the overlap function $\psi(E, \mathbf{p})$ is distinct from zero only for $p^{2}=E^{2}-\mathbf{p}^{2}$ within certain bounds (with $E>0$ ). On this interval, $\psi(E, \mathbf{p})$ is taken to be infinitely differentiable. In that case, the Jacob-Sachs theorem says that the asymptotic value $(T \rightarrow \infty)$ of the energy integral in Eq. (35) is given by its residue at the pole below the real axis. Thus, the evaluation of the partial amplitude $\mathcal{A}_{j}$ with the Jacob-Sachs theorem yields

$$
\mathcal{A}_{j} \cong \frac{\pi}{(2 \pi)^{4}} \int \frac{d^{3} p}{E_{j}(\mathbf{p})} \psi\left(E_{j}(\mathbf{p}), \mathbf{p}\right) e^{-i \phi_{j}(\mathbf{p})-\gamma_{j}(\mathbf{p})},
$$

where

$$
\begin{aligned}
E_{j}(\mathbf{p}) & =\sqrt{m_{j}^{2}+\mathbf{p}^{2}} \\
\phi_{j}(\mathbf{p}) & =E_{j}(\mathbf{p}) T-\mathbf{p} \cdot \mathbf{L} \\
\gamma_{j}(\mathbf{p}) & =\frac{m_{j} \Gamma_{j}}{2 E_{j}(\mathbf{p})} T .
\end{aligned}
$$

For Gaussian external wave packets, the overlap function is given by Eqs. (53)-(55). In principle, this function should be cut off outside the energy range determined by experimental conditions so as to satisfy the conditions of the Jacob-Sachs theorem. However these corrections are very small and will be neglected in the computations. The reader is referred to the Appendix for more details on the validity conditions of this theorem.

It is interesting to note that the amplitude (60) is mathematically equivalent to the amplitude obtained in the intermediate wave packet model [176], in which the mass eigenstates are directly represented by wave packets. The overlap function $\psi\left(E_{j}(\mathbf{p}), \mathbf{p}\right)$ corresponds to the wave function of the jth mass eigenstate. Thus it makes sense, in an external wave packet model, to talk about mass eigenstate 'wave packets' associated with the propagating particle.

This wave packet picture brings up a problem, as the overlap function takes into account not only the properties of the source, but also of the detector. This is unusual for a wave 
packet interpretation and not satisfying for causality. This point was recently clarified in an interesting article by Giunti 138 which was already mentioned in section 2.5. In this paper, Giunti proposes a sophisticated version of the intermediate wave packet model, in which the wave packet of the oscillating particle is explicitly computed with field-theoretical methods in terms of external wave packets. The intermediate wave packet depends only on the function $\psi_{P}\left(p^{0}, \mathbf{p}\right)$ (see Eq. (54)). The translation of this wave packet to the detection point yields a factor $\exp \left[-i \phi_{j}(\mathbf{p})\right]$. At the detector, its interaction with external wave packets gives rise to the function $\psi_{D}\left(p^{0}, \mathbf{p}\right)$. Remarkably, the total amplitude is exactly the same as Eq. (60). However the propagating wave packet now depends only on the properties of the source through the function $\psi_{P}\left(p^{0}, \mathbf{p}\right)$. The only drawbacks of this model are that it needs the equal time prescription and that it is not applicable to unstable oscillating particles.

\subsection{Three propagation regimes}

\subsubsection{Laplace's and stationary phase methods}

Unfortunately, the integration over the 3-momentum in Eq. (60) cannot be done analytically. Resorting to the explicit form (53)-(55) of the overlap function valid for Gaussian external wave packets, we see that the integral (60) can be approximated by means of an asymptotic expansion for which two kinds of large parameters can be used. On the one hand, $\sigma_{p P, D}^{-2}$ and $\sigma_{e P, D}^{-2}$ are large parameters appearing in the overlap function (53)-(55). They suggest a second order expansion of the integrand around the maximum $\mathbf{p}_{j}$ of the overlap function, followed by a Gaussian integration: this is called Laplace's method [111, 53]. On the other hand, $T$ and $\mathbf{L}$ are large parameters appearing in the phase. They suggest a second order expansion of the integrand around the stationary point $\mathbf{p}_{c l, j}$ of the phase, followed by a Gaussian integration: this is called the method of stationary phase [111, 53]. The competition between these two asymptotic behaviors implies a detailed study of the oscillation of the phase around the average momentum $\mathbf{p}_{j}$ (Fig. 4). The expansion of the phase in Eq. (60) should be compared with the expansion of the overlap function. Although both methods are expected to lead roughly to the same answer in the case of the propagation of a single particle, it should be checked whether the delicate compensation mechanism resulting in the oscillation phase is independent of the method chosen.

In section 5.3.2, it will be shown that Laplace's method is preferable if the dispersion (i.e. spreading in time) of the amplitude is negligible. This is certainly the case sufficiently near the source but is not true anymore beyond some threshold. From the discussion of section 4.5.1, it is expected that the spreading of the amplitude first begins in directions transversal to the propagation axis, before becoming significant along this axis. It is thus appropriate to divide the propagation range into three regimes: the no-dispersion regime, the transversal-dispersion regime and the longitudinal-dispersion regime. Laplace's method will be used in the first regime in all directions and in the second regime along the propagation axis, whereas the method of stationary phase will be used in the second regime along transversal directions and in the third 


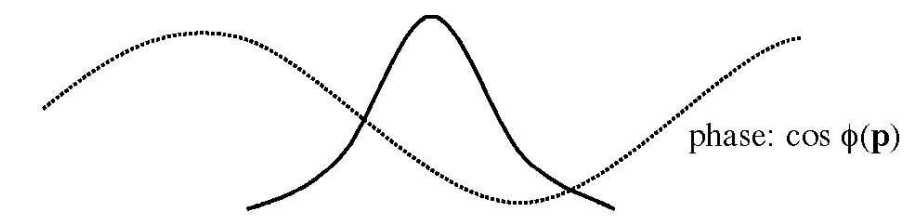

overlap function: $\psi\left(\mathrm{E}_{\mathrm{j}}(\mathbf{p}), \mathbf{p}\right)$

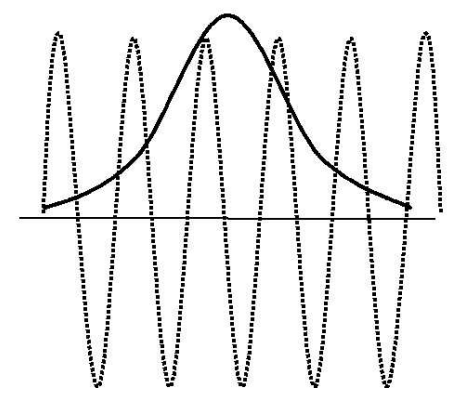

Figure 4: Laplace's method is preferable if the phase varies slowly (upper drawing), whereas the method of stationary phase is preferable if the phase varies rapidly (lower drawing). The overlap function (resp. the cosine of the phase) is indicated on both drawings by a continuous (resp. dotted) line.

regime in all directions.

As the phase of an amplitude corresponding to a propagating particle oscillates in $p L$, the interference between two amplitudes corresponding to two mass eigenstates provides a phase of the form $\epsilon p L$, where $\epsilon=\delta m_{i j}^{2} / 2 p_{0}^{2}$, with $\delta m_{i j}^{2}=m_{i}^{2}-m_{j}^{2}$. When the conditions of production and detection of the oscillating particle are studied with the help of Gaussian wave packets, a new parameter is introduced, namely $\sigma_{p}^{2}$. Therefore, the parameter $\epsilon$ can also appear with other dimensionless coefficients like $\left(p / \sigma_{p}\right)^{2}$ and $\left(\sigma_{p} L\right)^{2}$. These new coefficients are smaller than the original coefficient $p L$ if the distance $L$ is macroscopic. The new contributions will be neglected in the phase, since they are much smaller than the oscillating phase $\epsilon p L$. On the other hand, they should not be neglected in the modulus of the amplitude, where they are the leading terms in the $\epsilon$-expansion and yield observability conditions of oscillations.

We shall thus calculate the transition probability to $\mathcal{O}\left(\epsilon^{2}\right)$ in the real part of the argument of the exponential and to $\mathcal{O}(\epsilon)$ in the phase. Gaussian integrations will be consistent with the $\epsilon$ expansion if the extremum is computed to $\mathcal{O}(\epsilon)$, the real part of the argument of the exponential to $\mathcal{O}\left(\epsilon^{2}\right)$, the phase to $\mathcal{O}(\epsilon)$, the first derivatives to $\mathcal{O}(\epsilon)$ and the second derivatives to $\mathcal{O}\left(\epsilon^{0}\right)$.

\subsubsection{No-dispersion regime}

As discussed above, the integration over the 3-momentum in Eq. (60) is done by Laplace's method if the phase varies slowly over the width of the overlap function, i.e. if the particle is sufficiently near the source. The aim of this section is to determine the extension of this no-dispersion regime and to compute the amplitude $\mathcal{A}_{j}$ in this range. 
The first step consists in analyzing the form of the overlap function (53)-(55). The influence of the decay term $\gamma_{j}(\mathbf{p})$ (Eq. (63)) will be neglected in this section since we are near the source. The study of the overlap function amounts to study the argument of the exponential, i.e. of the function $f_{j}(\mathbf{p})$ defined by

$$
f_{j}(\mathbf{p})=f_{P}\left(E_{j}(\mathbf{p}), \mathbf{p}\right)+f_{D}\left(E_{j}(\mathbf{p}), \mathbf{p}\right),
$$

where $f_{P, D}$ are defined by Eq. (55) (with the constraint (59)) and $E_{j}(\mathbf{p})$ by Eq. (61). It is convenient to expand all results in small mass differences around $\left(E_{0}, \mathbf{p}_{0}, m_{0}, \mathbf{v}_{0}\right)$, where $m_{0}^{2}=E_{0}^{2}-\mathbf{p}_{0}^{2}$ and $\mathbf{v}_{0}=\mathbf{p}_{0} / E_{0}$. The expansion parameter is noted $\epsilon$ and refers collectively to all $\delta m_{j}^{2} / 2 E_{0}^{2}$, where $\delta m_{j}^{2}=m_{j}^{2}-m_{0}^{2}$. The value $\mathbf{p}_{j}$ minimizing $f_{j}(\mathbf{p})$ is given to $\mathcal{O}(\epsilon)$ by

$$
\mathbf{p}_{j}=\mathbf{p}_{0}+\left(\alpha \mathbf{u}_{P}+\beta \mathbf{u}_{D}\right) \frac{\delta m_{j}^{2}}{2 E_{0}}
$$

where

$$
\mathbf{u}_{P, D}=\frac{\mathbf{v}_{0}-\mathbf{v}_{P, D}}{2 \sigma_{e P, D}} .
$$

The associated energy $E_{j}=\sqrt{\mathbf{p}_{j}^{2}+m_{j}^{2}}$ can be expanded to $\mathcal{O}(\epsilon)$ as

$$
E_{j}=E_{0}+\tilde{\rho} \frac{\delta m_{j}^{2}}{2 E_{0}}
$$

where $\tilde{\rho}=1+\alpha \mathbf{v}_{0} \cdot \mathbf{u}_{P}+\beta \mathbf{v}_{0} \cdot \mathbf{u}_{D}$. The values of the dimensionless coefficients $\alpha$ and $\beta$ can be computed but their explicit expressions will not be needed. It is sufficient to know that $\tilde{\rho} \rightarrow 0$ in the stationary limit. A velocity $\mathbf{v}_{j}=\mathbf{p}_{j} / E_{j}$ is also defined for future use.

We are now going to approximate the overlap function as a Gaussian and compute its three characteristic widths. At the extremum $\mathbf{p}_{j}$, the Hessian matrix of $f_{j}(\mathbf{p})$ reads to $\mathcal{O}\left(\epsilon^{0}\right)$

$$
\begin{aligned}
\Sigma^{a b} & \equiv \frac{1}{2} \frac{\partial^{2} f_{j}}{\partial p^{a} \partial p^{b}}\left(\mathbf{p}_{j}\right) \\
& =\frac{\delta^{a b}}{4 \sigma_{p}^{2}}+u_{P}^{a} u_{P}^{b}+u_{D}^{a} u_{D}^{b}
\end{aligned}
$$

where the momentum width $\sigma_{p}$ is defined by

$$
\frac{1}{\sigma_{p}^{2}}=\frac{1}{\sigma_{p P}^{2}}+\frac{1}{\sigma_{p D}^{2}}
$$

and is approximately equal to the smallest width among the production and detection momentum widths. The associated width $\sigma_{x}$ in configuration space is defined by $\sigma_{p} \sigma_{x}=1 / 2$. The matrix $\Sigma^{a b}$ determines the range of $\mathbf{p}$ values for which the overlap function $\psi\left(E_{j}(\mathbf{p})\right.$, $\left.\mathbf{p}\right)$ is not negligible. As $\Sigma^{a b}$ is symmetric, it can be diagonalized by an orthogonal coordinate transformation. The eigenvalues of $\Sigma^{a b}$ are

$$
\begin{aligned}
\sigma_{x}^{2} & =\frac{1}{4 \sigma_{p}^{2}} \\
\sigma_{x \pm}^{2} & =\frac{1}{4 \sigma_{p}^{2}}+\frac{1}{2}\left(\mathbf{u}_{P}^{2}+\mathbf{u}_{D}^{2}\right) \pm \frac{1}{2} \sqrt{\left(\mathbf{u}_{P}^{2}+\mathbf{u}_{D}^{2}\right)^{2}-4\left(\mathbf{u}_{P} \times \mathbf{u}_{D}\right)^{2}} .
\end{aligned}
$$


The eigenvector associated with $\sigma_{x}^{2}$ is in the direction of $\mathbf{u}_{P} \times \mathbf{u}_{D}$, whereas the eigenvectors associated with $\sigma_{x \pm}^{2}$ belong to the plane defined by $\mathbf{u}_{P}$ and $\mathbf{u}_{D}$. In the limit $\left|\mathbf{u}_{P}\right| \gg\left|\mathbf{u}_{D}\right|$ (resp. $\left.\left|\mathbf{u}_{P}\right| \ll\left|\mathbf{u}_{D}\right|\right)$, the eigenvalues $\sigma_{x}^{2}$ and $\sigma_{x-}^{2}$ become degenerate and the eigenvector associated with $\sigma_{x+}^{2}$ becomes aligned with $\mathbf{u}_{P}$ (resp. $\mathbf{u}_{D}$ ). This is also the case in the limit of parallel $\mathbf{u}_{P}$ and $\mathbf{u}_{D}$. These limits are relevant to the case of stationary boundary conditions which are examined below.

Let us choose coordinate axes $\left(\mathbf{e}_{x}, \mathbf{e}_{y}, \mathbf{e}_{z}\right)$ coinciding with the normalized eigenvectors associated with $\left(\sigma_{x}^{2}, \sigma_{x-}^{2}, \sigma_{x+}^{2}\right)$ respectively. The quantities $\left(\sigma_{p}^{2}, \sigma_{p-}^{2}, \sigma_{p+}^{2}\right)\left(\right.$ with $\left.\sigma_{p \pm} \sigma_{x \pm}=1 / 2\right)$ can be interpreted as the momentum widths of the overlap function, since they give constraints on the range of $\mathbf{p}$ values for which the overlap function is non-negligible:

$$
\begin{aligned}
\left|p^{x}-p_{j}^{x}\right| & \lesssim \sigma_{p}, \\
\left|p^{y}-p_{j}^{y}\right| & \lesssim \sigma_{p-}, \\
\left|p^{z}-p_{j}^{z}\right| & \lesssim \sigma_{p+} .
\end{aligned}
$$

The case of the stationary limit is of special interest since this assumption is used by several authors 187, 150, 151, 163, 87. Recall that stationary boundary conditions are obtained in the external wave packet model by taking $\mathbf{v}_{P, D} \rightarrow 0$ and $\sigma_{e P, D} \rightarrow 0$ with $\left|\mathbf{v}_{P, D}\right| \lesssim \sigma_{e P, D} S_{P, D}$ (see Eq. (58)). In this limit, the velocity $\mathbf{v}_{0}$ becomes aligned with the axis $\mathbf{e}_{z}$,

$$
v_{0}^{x, y} \sim v_{P, D}^{x, y} \rightarrow 0
$$

and two eigenvalues become degenerate whereas the third diverges:

$$
\begin{aligned}
& \sigma_{x-}^{2} \rightarrow \sigma_{x}^{2}, \\
& \sigma_{x+}^{2} \rightarrow \frac{1}{4 \sigma_{p}^{2}}+\mathbf{u}_{P}^{2}+\mathbf{u}_{D}^{2} \rightarrow \infty
\end{aligned}
$$

In other words, the transversal widths (i.e. in directions orthogonal to $\mathbf{p}_{0}$ ) are given by $\sigma_{p}$ in the stationary limit, whereas the longitudinal width (i.e. in the direction of $\mathbf{p}_{0}$ ) is given by $\sigma_{p+} \rightarrow 0$.

The second step consists in comparing the expansion of the overlap function with the expansion of the phase $\phi_{j}(\mathbf{p})$ around $\mathbf{p}_{j}$, which reads

$$
\phi_{j}(\mathbf{p}) \cong \phi_{j}\left(\mathbf{p}_{j}\right)+\left(\mathbf{v}_{j} T-\mathbf{L}\right)\left(\mathbf{p}-\mathbf{p}_{j}\right)+\frac{T}{2 E_{0}}\left(p^{a}-p_{j}^{a}\right) R^{a b}\left(p^{b}-p_{j}^{b}\right),
$$

where $R^{a b}=\delta^{a b}-v_{0}^{a} v_{0}^{b}$. The second derivatives have been evaluated to $\mathcal{O}\left(\epsilon^{0}\right)$.

Laplace's method will be preferred to the method of stationary phase if the phase $\phi_{j}(\mathbf{p})$ varies slowly over the 'bump' of the overlap function. As $\sigma_{p} \geq \sigma_{p-} \geq \sigma_{p+}$, the variation of the phase will become important in the first place along the direction $p^{x}$, then in the direction $p^{y}$ and finally in the direction $p^{z}$. The criterion for the use of Laplace's method in all three directions $p^{x, y, z}$ will thus be determined by considering the largest momentum width $\sigma_{p}$. The insertion of the constraints (69) into the phase (72) yields first order conditions for a slowly varying phase,

$$
\left|v_{j}^{x} T-L^{x}\right| \sigma_{p} \lesssim 1
$$




$$
\begin{aligned}
\left|v_{j}^{y} T-L^{y}\right| \sigma_{p-} & \lesssim 1, \\
\left|v_{j}^{z} T-L^{z}\right| \sigma_{p+} & \lesssim 1,
\end{aligned}
$$

as well as a second order condition,

$$
\frac{T}{E_{0}} \sigma_{p}^{2} \lesssim 1
$$

where we have used the property $q^{a} R^{a b} q^{b} \leq \mathbf{q}^{2}$ and the fact that $\sigma_{p}$ is the largest width. For a given $T$ satisfying Eq. (74), it is always possible to find a range of $\mathbf{L}$ values so that conditions (73) are satisfied. For other $\mathbf{L}$ values, the amplitude is negligible as will be checked on the result (see Eq. (79)). Thus the criterion allowing to choose the integration method is determined by Eq. (74): the integration on $\mathbf{p}$ will be done by Laplace's method if $T \lesssim E_{0} / \sigma_{p}^{2}$ or, equivalently (with $L=|\mathbf{L}|$ and $p_{0}=\left|\mathbf{p}_{0}\right|$ ), if

$$
L \lesssim \frac{p_{0}}{\sigma_{p}^{2}}
$$

since conditions (73) impose the relation $\mathbf{L} \cong \mathbf{v}_{0} T$ as long as $\sigma_{x+} \ll L$.

For $\sigma_{x+} \gtrsim L$ (stationary limit), we now show that condition (75) is directly obtained without going through condition (74). The overlap function imposes in that limit that $|\mathbf{p}|=\sqrt{E_{0}^{2}-m_{j}^{2}}$, so that we are left with an angular integration with the angular part of the integrand given by

$$
\exp \left(\frac{\mathbf{p} \cdot \mathbf{p}_{0}}{2 \sigma_{p}^{2}}+i \mathbf{p} \cdot \mathbf{L}\right)
$$

Condition (75) shows that the angular variation of the phase in (76) is slow with respect to the angular variation of the overlap function, in which case Laplace's method will give good results. Therefore condition (75) constitutes a good criterion for the use of Laplace's method whether the stationary limit is taken or not.

Condition (75) is usually not verified in oscillation experiments, because $L / \sigma_{x} \gg p_{0} / \sigma_{p}$ in most cases. This condition is the same than the one under which Ioannisian and Pilaftsis 163] obtain 'plane wave' oscillations.

If condition (75) is satisfied, the evaluation of the integral (60) can be done by Laplace's method and yields

$$
\mathcal{A}_{j}=N \sigma_{p} \sigma_{p-} \sigma_{p+} \exp \left(-i E_{j} T+i \mathbf{p}_{j} \cdot \mathbf{L}-f_{j}\left(\mathbf{p}_{j}\right)-F_{j}(T)-\gamma_{j}\left(\mathbf{p}_{j}\right)\right),
$$

where $N$ absorbs numerical constants. The function $F_{j}(T)$ is defined by

$$
F_{j}(T)=\frac{1}{4}\left(\mathbf{v}_{j} T-\mathbf{L}\right)^{t}\left(\Sigma+i \frac{T}{2 E_{0}} R\right)^{-1}\left(\mathbf{v}_{j} T-\mathbf{L}\right),
$$

where $\Sigma^{a b}$ and $R^{a b}$ are considered as matrices. In the framework of the wave packet interpretation developed after Eq. (60), the function $\exp \left(-F_{j}(T)\right)$ plays the part of the space-time envelope of the wave packet associated with the jth mass eigenstate. The elements of the matrix $\operatorname{Re}(\Sigma+$ $\left.i \frac{T}{2 E_{0}} R\right)^{-1}$ constrain the extent of the wave packet envelope in space-time. As $T$ increases, the wave packet spreads because of the $i \frac{T}{E_{0}} R$ term. Thus the dispersion of the wave packet is due 
to the second order term in the expansion of the phase $\phi_{j}\left(\mathbf{p}_{j}\right)$. Therefore, condition (74) or, equivalently, condition (75) means that dispersion has not yet begun in any direction, transversal or longitudinal. For that reason, the range of $\mathbf{L}$ values defined by $L \lesssim p_{0} / \sigma_{p}^{2}$ is called the $n o$ dispersion regime. Note that this threshold was already met in the analysis of Gaussian wave packets in section 4.5.1 (see Eq. (44)). Of course, the above interpretation is not valid for $\sigma_{x+} \gtrsim L$, in which case the propagation time $T$ becomes indeterminate and dispersion loses its meaning.

Now that the origin of dispersion has been clarified, the term in Eq. (78) including $R$ can be neglected with respect to $\Sigma$. Moreover, we choose to approximate $\mathbf{v}_{j}$ by $\mathbf{v}_{0}$ in $F_{j}(T)$. In comparison with the oscillation formulas that will be derived in the transversal- and longitudinaldispersion regimes, this approximation will lead to the absence of the coherence-length term, since this term exclusively arises, when the dispersion is neglected, from the velocity difference $\mathbf{v}_{i}-\mathbf{v}_{j}$. Dropping the index $j$, the wave packet envelope in Eq. (77) can then be written in the coordinate system diagonalizing $\Sigma$ as

$$
F(T)=\frac{\left(v_{0}^{x} T-L^{x}\right)^{2}}{4 \sigma_{x}^{2}}+\frac{\left(v_{0}^{y} T-L^{y}\right)^{2}}{4 \sigma_{x-}^{2}}+\frac{\left(v_{0}^{z} T-L^{z}\right)^{2}}{4 \sigma_{x+}^{2}},
$$

which shows that the conditions (73) assumed for Laplace's method are required to obtain a non-negligible amplitude $\mathcal{A}_{j}$. In other words, $\mathbf{p}_{0}$ and $\mathbf{L}$ should be nearly parallel in order to have an amplitude significantly different from zero. We shall see that this constraint explicitly appears in the final oscillation formula (Eq. (111)).

\subsubsection{Transversal-dispersion regime}

Let us now assume that condition (75) is not satisfied, i.e. $L \gtrsim p_{0} / \sigma_{p}^{2}$. In that case, Laplace's method cannot be used to integrate on all three components $p^{x, y, z}$ in the amplitude (60) since dispersion becomes significant. However, the spreading of the amplitude is not identical in all directions. More specifically, the onset of dispersion in the direction $\mathbf{p}_{0}$ can be delayed by two factors. First, the matrix element $R^{a b}$ present in Eq. (72) leads to a relativistic contraction (of $1-\mathbf{v}_{0}^{2}$ ) in the direction $\mathbf{p}_{0}$ of the dispersion of the amplitude (see Eq. (78)). Second, the momentum width along $\mathbf{p}_{0}$ is given for $\sigma_{e P, D} \ll \sigma_{x}$ (i.e. in the stationary limit (71)) by a vanishing $\sigma_{p+}$. Thus Laplace's method is valid for a longer time $T$ in the direction $\mathbf{p}_{0}$ than in directions transverse to this vector. For this reason, the choice of the integration method in the direction $\mathbf{p}_{0}$ will be postponed for a short while, whereas the method of stationary phase will be preferred for momentum integrations in directions transverse to $\mathbf{p}_{0}$.

Let the $z$ axis be along $\mathbf{L}$, i.e. $\mathbf{L}=L \mathbf{e}_{z}$. As in section 5.3.2, the examination of the amplitude (60) shows that the quick variation of the phase averages the amplitude to zero unless $\mathbf{p}_{0}$ and $\mathbf{L}$ are nearly parallel. The method of stationary phase can thus be applied in directions $p^{x, y}$, the stationary points of which are given by $p^{x}=p^{y}=0$. The result of the method of stationary phase for the transverse momenta in (60) can be written as follows:

$$
\mathcal{A}_{j}=\frac{N g(\mathbf{l})}{T-i \mu} \int d p \exp \left(-i \phi_{j}(p)-f_{j}(p)-\gamma_{j}(p)\right)
$$


where $p \equiv p^{z}$ and

$$
\begin{aligned}
\phi_{j}(p) & =\sqrt{p^{2}+m_{j}^{2}} T-p L, \\
f_{j}(p) & =f_{j P}(p)+f_{j D}(p),
\end{aligned}
$$

with

$$
f_{j P}(p)=\frac{\left(p-p_{0}\right)^{2}}{4 \sigma_{p P}^{2}}+\frac{\left(\sqrt{p^{2}+m_{j}^{2}}-E_{0}-\left(p-p_{0}\right) v_{P}\right)^{2}}{4 \sigma_{e P}^{2}},
$$

where $v_{P}=v_{P}^{z}$ and $p_{0}=p_{0}^{z}$. The average energy $E_{0}$ has been redefined so as to absorb a factor $p_{0}^{x} v_{P}^{x}+p_{0}^{y} v_{P}^{y}$. The definition of $f_{j D}(p)$ is similar. Finally, $\gamma_{j}(p)$ expresses the possible decay of the oscillating particle:

$$
\gamma_{j}(p)=\frac{m_{j} \Gamma_{j} T}{2 \sqrt{p^{2}+m_{j}^{2}}} .
$$

The function $g(\mathbf{l})$ comes from the transversal part of the overlap function. It expresses the geometrical constraint between the direction of observation $\mathbf{l}=\mathbf{L} / L$ and the momentum $\mathbf{p}_{0}$ :

$$
g(\mathbf{l})=\exp \left(-\frac{\left(\mathbf{p}_{0} \times \mathbf{l}\right)^{2}}{4 \sigma_{p}^{2}}\right)
$$

where $\sigma_{p}$ is defined by Eq. (67). The function $g(\mathbf{l})$ restricts the propagation to a cone of axis $\mathbf{p}_{0}$ and angle $\arcsin \left(\sigma_{p} / p_{0}\right)$ (see Fig. 6 in section 7.1).

Numerical constants have been included in $N$, as well as 4 -volume factors $\sigma_{p P}^{-3} \sigma_{e P}^{-1}$ and $\sigma_{p D}^{-3} \sigma_{e D}^{-1}$, and an energy factor $E_{0}$. The constant $\mu=E_{0} / 2 \sigma_{p}^{2}$ comes from the overlap function and acts as a cut-off for small $T$. As $T$ is macroscopic, the prefactor $1 /(T-i \mu)$ can be approximated by $1 / T$ so as to give a prefactor $1 / L^{2}$ in the transition probability. This expected geometrical decrease is seen to originate in the transverse dispersion of the wave packet corresponding to the oscillating particle. As in section 5.3.2, it is convenient to define a reference mass $m_{0}$ and a velocity $v_{0}$ by

$$
\begin{aligned}
m_{0}^{2} & =E_{0}^{2}-p_{0}^{2} \\
v_{0} & =p_{0} / E_{0} .
\end{aligned}
$$

As before, the choice of the method to perform the longitudinal momentum integration (80) is done by comparing the expansions of the phase and of the overlap function around the value $p_{j}$ for which $f_{j}(p)+\gamma_{j}(p)$ is extremal (the decay term is not neglected in this section). The explicit value of $p_{j}$ will be computed in section 6.3 for the stable case and in section 8.2 for the unstable case.

The expansions of $f_{j}(p)$ and $\phi_{j}(p)$ are given by

$$
\begin{aligned}
f_{j}(p) \cong f_{j}\left(p_{j}\right)+\frac{\left(p-p_{j}\right)^{2}}{4 \sigma_{\text {peff }}^{2}} \\
\phi_{j}(p) \cong \phi_{j}\left(p_{j}\right)+\left(v_{j} T-L\right)\left(p-p_{j}\right)+\frac{m_{j}^{2} T}{2 E_{0}^{3}}\left(p-p_{j}\right)^{2},
\end{aligned}
$$


where $E_{j}=\sqrt{p_{j}^{2}+m_{j}^{2}}$ and $v_{j}=p_{j} / E_{j}$.

The effective width $\sigma_{p e f f}$ and $\sigma_{x e f f}$ are defined by

$$
\begin{aligned}
& \frac{1}{\sigma_{p e f f}^{2}}=\frac{1}{\sigma_{p P}^{2}}+\frac{1}{\sigma_{p D}^{2}}+\frac{\left(v_{0}-v_{P}\right)^{2}}{\sigma_{e P}^{2}}+\frac{\left(v_{0}-v_{D}\right)^{2}}{\sigma_{e D}^{2}}, \\
& \sigma_{p e f f} \sigma_{x e f f}=\frac{1}{2}
\end{aligned}
$$

Note that $\sigma_{p e f f}$ has the same form as the asymptotic value of $\sigma_{p+}$ (see Eq. (71)).

With the help of the wave packet correspondence discussed at the end of section 5.2, the effective width can be interpreted as the energy-momentum width of the oscillation process, since it is the width of the overlap function. It is dominated by the smallest among the energy uncertainties (recall that $\sigma_{e P, D} \leq \sigma_{p P, D}$ ). The effective width $\sigma_{x e f f}$ is then approximately equal either to the production or to the detection time uncertainty, depending on which one is the largest.

Laplace's method is preferable if the phase (88) varies slowly over the width $\sigma_{p e f f}$, i.e. if the two following conditions are satisfied

$$
\begin{aligned}
\left|v_{j} T-L\right| 2 \sigma_{p e f f} & \lesssim 1 \\
\frac{m_{j}^{2} T}{2 E_{0}^{3}} 4 \sigma_{p e f f}^{2} & \lesssim 1 .
\end{aligned}
$$

As in section 5.3.2, the first order constraint (90) will be included in the result of Laplace's method. Thus the criterion allowing to choose between Laplace and stationary phase methods is given by Eq. (91). In other words, it is better to use Laplace's method if $T$ is smaller than a dispersion time $T_{j}^{\text {disp }}$ defined by

$$
T_{j}^{d i s p}=\frac{E_{0}^{3}}{2 m_{j}^{2} \sigma_{p e f f}^{2}} .
$$

The term 'dispersion time' is justified by the fact that it is the time at which the longitudinal dispersion of the amplitude becomes important, more precisely twice the initial size. A dispersion length $L_{j}^{\text {disp }}$ can be defined by $L_{j}^{d i s p}=v_{0} T_{j}^{d i s p}$. The distance range $p_{0} / \sigma_{p}^{2} \lesssim L \lesssim L_{j}^{\text {disp }}$ will be called the tranversal-dispersion regime. For $L \gtrsim L_{j}^{\text {disp }}$, the stationary phase method is more accurate: this distance range will be called the longitudinal-dispersion regime. Note that this threshold was already met in the analysis of Gaussian wave packets in section 4.5.1 (see Eq. (45)). Various estimates of the dispersion length are discussed in section 5.4.2, showing that the concept of dispersion length is relevant to nonrelativistic particles such as $K$ and $B$ mesons, as well as to supernova neutrinos, and possibly to solar neutrinos.

In the transversal-dispersion regime, the evaluation of the amplitude (80) as a Gaussian integral around $p_{j}$ gives

$$
\mathcal{A}_{j}=\frac{N g(\mathbf{l}) \sigma_{\text {peff }}}{T \sqrt{1+i T / T_{j}^{\text {disp }}}} \exp \left(-i E_{j} T+i p_{j} L-f_{j}\left(p_{j}\right)-\gamma_{j}\left(p_{j}\right)-\frac{1}{1+i T / T_{j}^{\text {disp }}} \frac{\left(v_{j} T-L\right)^{2}}{4 \sigma_{\text {xeff }}^{2}}\right)
$$


where $N$ absorbs numerical constants and $p_{j}$ is the value for which $f_{j}(p)+\gamma_{j}(p)$ is extremal.

The amplitude (93) behaves as a wave packet of group velocity $v_{j}$ and space-time extent $\left(1+\left(T / T_{j}^{d i s p}\right)^{2}\right)^{\frac{1}{2}} \sigma_{x e f f}$. If the longitudinal dispersion is neglected $\left(T_{j}^{d i s p}=\infty\right)$, the amplitude (93) is similar to Eq. (18) of Ref. 144.

\subsubsection{Longitudinal-dispersion regime}

At sufficiently large distance, dispersion becomes significant and all neutrinos propagating freely enter into the longitudinal-dispersion regime. In this regime $\left(L \gtrsim L_{j}^{d i s p}\right)$, it has been argued that the integral (80) should be evaluated with the method of stationary phase. The stationary point of the phase $\phi_{j}(p)$ is given by

$$
p_{c l, j}=m_{j} \frac{v_{c l}}{\sqrt{1-v_{c l}^{2}}}
$$

where $v_{c l}=L / T$. It can be interpreted as the classical momentum of a particle of mass $m_{j}$, travelling at the classical velocity $v_{c l}$. Of course, a stationary point exists only for $T \geq L$. Otherwise Laplace's method must be used, but the amplitude is nearly zero in that case anyway. The evaluation of the amplitude (80) as a Gaussian integral around $p_{c l, j}$ gives

$$
\mathcal{A}_{j}=\frac{N g(\mathbf{l}) \sigma_{p e f f}}{T \sqrt{1+i T / T_{j}^{d i s p}}} \exp \left(-i m_{j} \sqrt{T^{2}-L^{2}}-f_{j}\left(p_{c l, j}\right)-\gamma_{j}\left(p_{c l, j}\right)+\sigma_{p e f f}^{2} \frac{\left(f_{j}^{\prime}\left(p_{c l, j}\right)\right)^{2}}{1+i T / T_{j}^{d i s p}}\right)
$$

where $f_{j}(p)$ is defined by Eq. (82), $f_{j}^{\prime}(p)$ refers to its derivative and $N$ absorbs numerical constants. The wave packet interpretation of the amplitude (95) is not obvious but the shape of the associated wave packet can be studied by an expansion around the maximum of the amplitude.

\subsection{Estimates of the dispersion thresholds}

In section 5.3, it was shown that the propagation range could be divided into three regimes separated by two thresholds (Fig. 5). The first threshold is determined by the no-dispersion condition (75) and separates the no-dispersion regime from the transversal-dispersion regime at $L^{\text {nodisp }}=p_{0} / \sigma_{p}^{2}$. The second threshold (see Eq. (92)) is given by the dispersion length $L_{j}^{d i s p}=p_{0} E_{0}^{2} / 2 m_{j}^{2} \sigma_{\text {peff }}^{2}$ and separates the transversal-dispersion regime from the longitudinaldispersion regime. It is not straightforward to estimate the value of these thresholds, mainly because of our ignorance of the external wave packet sizes.

\subsubsection{No-dispersion condition}

The first threshold is rarely of the order of a macroscopic distance. A far-fetched example where the propagation distance could be smaller than the no-dispersion threshold $L^{\text {nodisp }}=p_{0} / \sigma_{p}^{2}$ is given by Ioannisian and Pilaftsis [163]: atmospheric neutrinos are detected in the no-dispersion regime if $\sigma_{x} \gtrsim 10^{-2} \mathrm{~cm}$ (with $L \sim 1000 \mathrm{~km}$ and $p \sim 1 \mathrm{GeV}$ ). Although such a macroscopic size of 


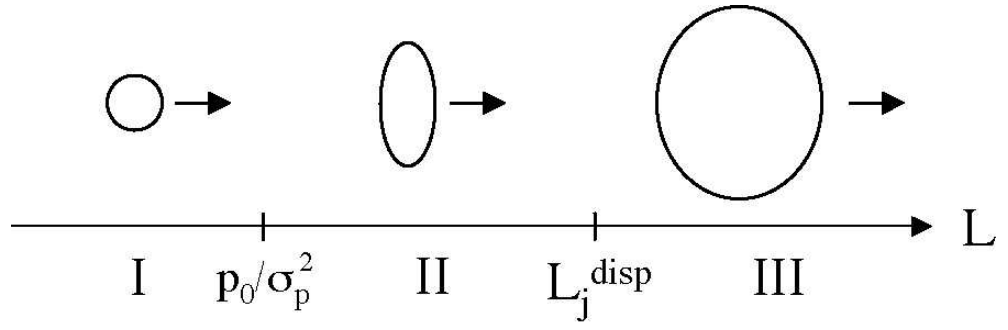

Figure 5: The propagation range can be divided into three regimes according to the dispersion.

the neutrino 'wave packet' is not totally excluded (see section 5.4.2), it is unlikely to be verified. However this case will be examined in detail in section 6.1 both for reason of completeness and because it was argued in Ref. [163] that the corresponding oscillation formula would differ significantly from the standard result.

\subsubsection{Dispersion length}

This threshold is a priori more interesting than the first, as it is of the order of a macroscopic distance. As said above, the main difficulty lies in the lack of knowledge of the external wave packet sizes. In the case of neutrinos, another uncertainty arises because of the dependence of the dispersion length on the absolute mass scale, instead of a dependence on the mass differences. Some examples are given below, partly based on the wave packet size discussion of section 9.2.2. We should always remember that $\sigma_{p e f f}$ is determined by the smallest width among the production and detection energy or momentum widths.

First, the neutrinos in the experiment LSND [28, 29] have a momentum around $30 \mathrm{MeV}$. The neutrino mass can be taken to be $1 \mathrm{eV}$, and the production and detection widths should be around $0.01 \mathrm{MeV}$ [152]. The corresponding dispersion length, around $10^{7} \mathrm{~m}$, is much longer than the $30 \mathrm{~m}$ length scale of the experiment. Second, atmospheric neutrinos have a momentum around $1 \mathrm{GeV}$ and let us take a mass of $1 \mathrm{eV}$ as a bound. An estimate of the source width can be obtained from the inverse pion and muon lifetimes and is given by $\sigma_{P P} \sim 10^{-14} \mathrm{MeV}$ in the case of a pion source. The detector width can be estimated at $\sigma_{x D} \sim 10^{-10} \mathrm{~m}$ [237], i.e. $\sigma_{p D} \sim 10^{-3} \mathrm{MeV}$, and is dominated by the source width. The corresponding dispersion length, larger than $10^{33} \mathrm{~km}$, is completely irrelevant to the experiment. It is however doubtful that the source width is truly estimated by the inverse source lifetime (see the discussion of section 9.2.2). Third, solar neutrinos have a momentum around $1 \mathrm{MeV}$ and let us take a mass of $1 \mathrm{eV}$ as a bound. Various estimates of the source width have been given, between $10^{-7} \mathrm{MeV}$ and $10^{-3} \mathrm{MeV}$ [216, 188, 200, 185, 182, 204]. The detector width can be estimated as above at $\sigma_{p D} \sim 10^{-3} \mathrm{MeV}$. The dispersion length, varying between $10^{2} \mathrm{~km}$ and $10^{10} \mathrm{~km}$ for a $1 \mathrm{eV}$ neutrino mass (depending on the chosen source width), could be relevant to the experiment, but is much larger for smaller neutrino masses.

As regards mesons, kaons in the CPLEAR experiment have a momentum around $550 \mathrm{MeV}$ 
[120]. If the width is guessed to be $0.01 \mathrm{MeV}$, the dispersion length, around $10^{-6} \mathrm{~m}$, is much smaller than the length scale of the experiment. More generally, the ratio between the dispersion length and the decay length (defined by $L^{\text {decay }}=p / m \Gamma$ ) is given by

$$
\frac{L^{\text {disp }}}{L^{\text {decay }}}=\frac{E^{2}}{\sigma_{p e f f}^{2}} \frac{\Gamma}{2 m}
$$

so that dispersion occurs for all quasi-stable particles $(\Gamma \ll m)$, and in particular for $B$ mesons.

In conclusion, if the estimates above are truthful, dispersion cannot be ignored for $K$ and $B$ mesons, and might be important for solar neutrinos (and certainly for supernova neutrinos). It is then necessary to develop a formalism taking into account the dispersion, in which case the use of the stationary phase method cannot be avoided.

\section{Oscillations of stable particles}

In section 5, the choice of the method of evaluation of the amplitude (35) was seen to depend on the distance range within which the oscillating particle detected. Three distance ranges were distinguished, depending on whether the amplitude had begun to spread in the transverse and longitudinal directions (with respect to the direction of propagation). The amplitude was then evaluated in the three possible cases, yielding formulas (77), (93) and (95) corresponding to the no-dispersion, transversal- and longitudinal-dispersion regimes respectively.

In this section[3], we compute the transition probability for stable oscillating particles. The transition probability is then obtained by squaring the amplitude and averaging over the macroscopic propagation time $T$, which is not observed in experiments:

$$
\mathcal{P}_{\alpha \rightarrow \beta}(\mathbf{L}) \sim \sum_{i, j} V_{i \alpha} V_{\beta i}^{-1} V_{j \alpha}^{*} V_{\beta j}^{-1 *} \int d T \mathcal{A}_{i} \mathcal{A}_{j}^{*} .
$$

We shall come back to the proportionality factor in sections 7 and 9.1.

The oscillation formula is derived in the three different propagation regimes in sections 6.1, 6.2 and 6.3. A shorter method of integration, valid for stable oscillating particles in the second and third regimes, is then discussed in section 6.4.

\subsection{Oscillations without dispersion}

\subsubsection{Amplitude}

For $L \lesssim p_{0} / \sigma_{p}^{2}$, the momentum integration in Eq. (60) is done by Laplace's method, yielding for stable oscillating particles (see Eq. (77) with $\gamma_{j}\left(\mathbf{p}_{j}\right)=0$ ) the following amplitude:

$$
\mathcal{A}_{j}=N \sigma_{p} \sigma_{p-} \sigma_{p+} \exp \left(-i E_{j} T+i \mathbf{p}_{j} \cdot \mathbf{L}-\left(\frac{\delta m_{j}^{2}}{4 \tilde{\sigma}_{m} E_{0}}\right)^{2}-F(T)\right),
$$

\footnotetext{
${ }^{13}$ The reader not interested in all computational details can skip this section. Its results are summarized in section 0 .
} 
where $\mathbf{p}_{j}$ is given by Eq. (65), and

$$
F(T)=\frac{\left(v_{0}^{x} T-L^{x}\right)^{2}}{4 \sigma_{x}^{2}}+\frac{\left(v_{0}^{y} T-L^{y}\right)^{2}}{4 \sigma_{x-}^{2}}+\frac{\left(v_{0}^{z} T-L^{z}\right)^{2}}{4 \sigma_{x+}^{2}},
$$

with $\sigma_{x}, \sigma_{x \pm}$ defined by Eqs. (68). The parameter $\tilde{\sigma}_{m}$, which has the dimension of a width, is related to $f_{j}\left(\mathbf{p}_{j}\right)$ (see Eq. (64)) by

$$
f_{j}\left(\mathbf{p}_{j}\right)=\left(\frac{\delta m_{j}^{2}}{4 \tilde{\sigma}_{m} E_{0}}\right)^{2}
$$

\subsubsection{Probability}

The last step towards the oscillation formula consists in computing the time average of the transition probability, which is a Gaussian integral on $T$ :

$$
\int d T \mathcal{A}_{i} \mathcal{A}_{j}^{*}=N_{\tilde{g}} \exp \left(-i \phi_{i j}\left(T_{0}\right)-\frac{\left(\delta m_{i}^{2}\right)^{2}+\left(\delta m_{j}^{2}\right)^{2}}{16 \tilde{\sigma}_{m}^{2} E_{0}^{2}}-\frac{\left(E_{i}-E_{j}\right)^{2}}{4 F^{\prime \prime}\left(T_{0}\right)}-2 F\left(T_{0}\right)\right),
$$

where $T_{0}$ is the solution of $F^{\prime}\left(T_{0}\right)=0$, while the phase $\phi_{i j}\left(T_{0}\right)$ is given by

$$
\phi_{i j}\left(T_{0}\right)=\left(E_{i}-E_{j}\right) T_{0}-\left(\mathbf{p}_{i}-\mathbf{p}_{j}\right) \cdot \mathbf{L} .
$$

Non-exponential factors are included in $N_{\tilde{g}}$. The function $F(T)$ can be rewritten as

$$
F(T)=\frac{\left(\tilde{\mathbf{v}}_{0} T-\tilde{\mathbf{L}}\right)^{2}}{4 \sigma_{x}^{2}}
$$

where

$$
\begin{aligned}
\tilde{\mathbf{v}}_{0} & =\sigma_{x} \sqrt{\Sigma^{-1}} \mathbf{v}_{0}, \\
\tilde{\mathbf{L}} & =\sigma_{x} \sqrt{\Sigma^{-1}} \mathbf{L},
\end{aligned}
$$

with $\Sigma^{-1}=\operatorname{diag}\left(\sigma_{x}^{-2}, \sigma_{x-}^{-2}, \sigma_{x+}^{-2}\right)$. It is then easy to compute

$$
\begin{aligned}
T_{0} & =\frac{\tilde{\mathbf{v}}_{0} \cdot \tilde{\mathbf{L}}}{\tilde{\mathbf{v}}_{0}^{2}} \\
F\left(T_{0}\right) & =\frac{\left(\tilde{\mathbf{v}}_{0} \times \tilde{\mathbf{L}}\right)^{2}}{4 \sigma_{x}^{2} \tilde{\mathbf{v}}_{0}^{2}} \\
F^{\prime \prime}\left(T_{0}\right) & =\frac{\tilde{\mathbf{v}}_{0}^{2}}{2 \sigma_{x}^{2}} .
\end{aligned}
$$

Actually, the time interval of integration $\Delta T$ is finite. The approximation $\Delta T \rightarrow \infty$ is good if $\Delta T$ is larger than the width of the overlap function given by Eq. (104):

$$
\left|\mathbf{v}_{0}\right| \Delta T \gtrsim \tilde{\sigma}_{x e f f}
$$

where

$$
\tilde{\sigma}_{x e f f}=\frac{\left|\mathbf{v}_{0}\right|}{\left|\tilde{\mathbf{v}}_{0}\right|} \sigma_{x}
$$


The insertion of Eq. (103) in Eq. (99) shows that $F\left(T_{0}\right)$ plays the role of a directional constraint since $\int d T \mathcal{A}_{i} \mathcal{A}_{j}^{*}$ is non-negligible if $\tilde{\mathbf{L}}$ is nearly parallel to $\tilde{\mathbf{v}}_{0}$. In order to express this constraint in terms of non-tilded quantities, two cases should be distinguished: $\sigma_{x+} \sim \sigma_{x}$ and $\sigma_{x+} \gg \sigma_{x}$.

For $\sigma_{x+} \sim \sigma_{x}, \int d T \mathcal{A}_{i} \mathcal{A}_{j}^{*}$ is non-negligible if $\mathbf{L}$ is nearly parallel to $\mathbf{v}_{0}$ :

$$
\mathbf{L}=\frac{\mathbf{v}_{0}}{\left|\mathbf{v}_{0}\right|} L+\mathcal{O}\left(\sigma_{x+}\right) .
$$

With the substitution (107), the value of $T_{0}$ given by Eq. (102) becomes $T_{0}=L /\left|\mathbf{v}_{0}\right|+\mathcal{O}\left(\sigma_{x+}\right)$, so that the phase (100) becomes

$$
\phi_{i j}\left(T_{0}\right)=\frac{\delta m_{i j}^{2}}{2 p_{0}}\left(L+\mathcal{O}\left(\sigma_{x+}\right)\right),
$$

which is equal to the standard oscillation phase (14) if $\sigma_{x+} \ll L$.

If $\sigma_{x+} \gg \sigma_{x}, \tilde{\mathbf{v}}_{0}$ and $\tilde{L}^{z}$ tend to zero so that $F\left(T_{0}\right)$ should directly be studied as a function of $\mathbf{v}_{0}$ and $\mathbf{L}$. General conclusions for arbitrary $\sigma_{x \pm}$ can be drawn from the study of the quadratic form in $\left(L^{x}, L^{y}, L^{z}\right)$ associated with $F\left(T_{0}\right)=1$. This analysis shows that there is an eigenvalue $s_{3}=0$ corresponding to an eigenvector along $\mathbf{v}_{0}$. The two other eigenvalues $s_{1,2}$ are positive (with $\left.s_{2} \leq s_{1}\right)$, so that the surface $F\left(T_{0}\right)=1$ in $\left(L^{x}, L^{y}, L^{z}\right)$-space is a cylinder of elliptical section with an axis along $\mathbf{v}_{0}$. This geometrical picture can be interpreted as imposing that the components of $\mathbf{L}$ orthogonal to $\mathbf{v}_{0}$ should be smaller than $\sqrt{1 / s_{2}}$, whereas there is no constraint at all on the component of $\mathbf{L}$ along $\mathbf{v}_{0}$. In other words, the probability is maximal within a cylinder of axis $\mathbf{v}_{0}$.

In the stationary limit $\left(\sigma_{x+} \rightarrow \infty\right)$, the lengthy expressions of the non-zero eigenvalues become

$$
\begin{aligned}
& s_{1} \rightarrow \frac{1}{4 \sigma_{x}^{2}}, \\
& s_{2} \rightarrow \frac{1}{4 \sigma_{x}^{2}} \frac{\sigma_{x}^{2}\left(v_{0}^{z}\right)^{2}}{\sigma_{x+}^{2}\left(v_{0}^{x}\right)^{2}+\sigma_{x+}^{2}\left(v_{0}^{y}\right)^{2}+\sigma_{x}^{2}\left(v_{0}^{z}\right)^{2}} .
\end{aligned}
$$

The properties of the stationary limit, namely Eqs. (58), (70) and (71), lead to the bound $\sqrt{1 / s_{2}} \lesssim S_{P, D}$. In the stationary limit, the components $L^{x, y}$ (which are then orthogonal to $\mathbf{v}_{0}$ ) should thus be smaller than $S_{P, D}$. The time-averaged probability is therefore always negligible in directions other than along the average momentum $\mathbf{p}_{0}$. Thus the evaluation of the phase $\phi_{i j}\left(T_{0}\right)$ yields

$$
\phi_{i j}\left(T_{0}\right)=\frac{\delta m_{i j}^{2}}{2 p_{0}}\left(L+\mathcal{O}\left(S_{P, D}\right)\right),
$$

which is equal to the standard oscillation phase (14) if $S_{P, D} \ll L$.

With the results (99), (103), (104) and 108) (or (110)), the flavor-mixing transition probability (96) can be written for a propagation distance satisfying $S_{P, D} \ll L \lesssim p_{0} / \sigma_{p}^{2}$ as

$$
\mathcal{P}_{\alpha \rightarrow \beta}(\mathbf{L}) \sim N_{\tilde{g}} \exp \left(-\frac{\left(\tilde{\mathbf{v}}_{0} \times \tilde{\mathbf{L}}\right)^{2}}{2 \sigma_{x}^{2} \tilde{\mathbf{v}}_{0}^{2}}\right) \sum_{i, j} V_{i \alpha} V_{i \beta}^{*} V_{j \alpha}^{*} V_{j \beta}
$$




$$
\times \exp \left(-2 \pi i \frac{L}{L_{i j}^{o s c}}-\frac{\left(\delta m_{i}^{2}\right)^{2}+\left(\delta m_{j}^{2}\right)^{2}}{16 \tilde{\sigma}_{m}^{2} E_{0}^{2}}-2 \pi^{2}\left(\frac{\tilde{\rho} \tilde{\sigma}_{x e f f}}{L_{i j}^{o s c}}\right)^{2}\right),
$$

where $\tilde{\rho}$ and $\tilde{\sigma}_{x e f f}$ are defined by Eqs. (66) and (106), respectively. $N_{\tilde{g}}$ includes all prefactors independent of $L$ as well as normalization factors. The oscillation length $L_{i j}^{o s c}$ is defined by

$$
L_{i j}^{o s c}=\frac{4 \pi\left|\mathbf{p}_{0}\right|}{\delta m_{i j}^{2}}
$$

As discussed above, the probability is maximal within a cylinder of axis $\mathbf{v}_{0}$ and radius smaller than $S_{P, D}$. There is no decrease in $1 / L^{2}$ since the spatial spreading of the 'wave packet' has not yet begun.

\subsection{Oscillations with transversal dispersion}

\subsubsection{Amplitude}

For $p_{0} / \sigma_{p}^{2} \lesssim L \lesssim L_{j}^{d i s p}$, the momentum integration in Eq. (60) is done by the method of stationary phase in the transverse directions and by Laplace's method in the longitudinal direction, yielding in the stable case (see Eq. (93) with $\gamma_{j}\left(p_{j}\right)=0$ )

$$
\mathcal{A}_{j}=\frac{N g(\mathbf{l}) \sigma_{\text {peff }}}{T \sqrt{1+i T / T_{j}^{\text {disp }}}} \exp \left(-i E_{j} T+i p_{j} L-f_{j}\left(p_{j}\right)-\frac{1}{1+i T / T_{j}^{\text {disp }}} \frac{\left(v_{j} T-L\right)^{2}}{4 \sigma_{\text {xeff }}^{2}}\right),
$$

with $f_{j}(p)$ and $\sigma_{p e f f}$ defined by Eqs. (82) and (89), respectively. We had not yet determined the value $p_{j}$ for which $f_{j}(p)$ is extremal. The computation of $p_{j}$ to $\mathcal{O}(\epsilon)$ yields

$$
p_{j}=p_{0}+(\rho-1) \frac{\delta m_{j}^{2}}{2 p_{0}}
$$

where the dimensionless number $\rho$ is defined by

$$
\rho=\sigma_{p e f f}^{2}\left(\frac{1}{\sigma_{p}^{2}}-\frac{v_{P}\left(v_{0}-v_{P}\right)}{\sigma_{e P}^{2}}-\frac{v_{D}\left(v_{0}-v_{D}\right)}{\sigma_{e D}^{2}}\right),
$$

with $\sigma_{p}$ defined by Eq. (67). The associated energy $E_{j}=\sqrt{p_{j}^{2}+m_{j}^{2}}$ and velocity $v_{j}=p_{j} / E_{j}$ are given to $\mathcal{O}(\epsilon)$ by

$$
\begin{aligned}
E_{j} & =E_{0}+\rho \frac{\delta m_{j}^{2}}{2 E_{0}} \\
v_{j} & =v_{0}+\left(\rho\left(1-v_{0}^{2}\right)-1\right) \frac{\delta m_{j}^{2}}{2 p_{0} E_{0}} .
\end{aligned}
$$

The parameter $\rho$ has been defined so as to be in correspondence with the notation of Ref. 144]. The symbol $\omega$ appearing in that article is related to our notation by $\omega=\sigma_{p}^{2} / \sigma_{\text {peff }}^{2}$. Note that the authors of Ref. [144] do not compute $\rho$ explicitly and also take the relativistic limit $v_{0}=1$. 
The explicit value of $\rho$ is very interesting to know, since $\rho=0$ in the case of stationary boundary conditions, in which case all mass eigenstates have the same energy $E_{0}$.

The value of $f_{j}(p)$ at order $\epsilon^{2}$ is given by

$$
f_{j}\left(p_{j}\right)=\frac{1}{4 \sigma_{m}^{2}}\left(\frac{\delta m_{j}^{2}}{2 E_{0}}\right)^{2}+\mathcal{O}\left(\epsilon^{3}\right)
$$

where $\sigma_{m}$ is defined by

$$
\frac{1}{\sigma_{m}^{2}}=\sigma_{p e f f}^{2}\left(\frac{1}{\sigma_{p}^{2}}\left(\frac{1}{\sigma_{e P}^{2}}+\frac{1}{\sigma_{e D}^{2}}\right)+\frac{\left(v_{P}-v_{D}\right)^{2}}{\sigma_{e P}^{2} \sigma_{e D}^{2}}\right) .
$$

$\sigma_{m}$ will be called the mass width, as it imposes a constraint between the masses $m_{i, j}$ and the ingoing energy-momentum (see section 7.1.5).

\subsubsection{Probability}

As in section 6.1.2, the last step towards the oscillation formula consists in computing the time average of the transition probability. Actually, the computation can be simplified by considering separately two cases. The examination of Eq. (118) shows indeed that the amplitude $\mathcal{A}_{j}$ (Eq. (113) ) is negligible unless $\left|\delta m_{j}^{2} / 2 E_{0}\right| \lesssim \sigma_{m}$. Together with the same condition on $\mathcal{A}_{i}$, it puts a constraint on the mass difference:

$$
\left(\frac{\delta m_{i j}^{2}}{2 E_{0}}\right)^{2} \lesssim 2 \sigma_{m}^{2} \quad \text { where } \quad \delta m_{i j}^{2}=m_{i}^{2}-m_{j}^{2} .
$$

There are two possible cases:

1. the masses are nearly degenerate;

2. if not, the masses must be very small in comparison with the energy $E_{0}$, i.e. the oscillating particles are relativistic.

We first consider the case of nearly degenerate masses. If $\left|m_{i}-m_{j}\right| \ll m_{i}, m_{j}$, it is possible to make the approximation $T_{i}^{d i s p} \cong T_{j}^{d i s p}$ and to work with only one dispersion time defined by $T^{d i s p}=E_{0}^{3} / 2 \tilde{m}_{0}^{2} \sigma_{\text {peff }}^{2}$, with $\tilde{m}_{0}$ the mass in the degenerate limit. The integrand in (96) can be written as

$$
\mathcal{A}_{i} \mathcal{A}_{j}^{*}=\frac{N^{2} g^{2}(\mathbf{l}) \sigma_{\text {peff }}^{2}}{T^{2} \sqrt{1+\left(T / T^{d i s p}\right)^{2}}} \exp \left(-\frac{\left(\delta m_{i}^{2}\right)^{2}+\left(\delta m_{j}^{2}\right)^{2}}{16 \sigma_{m}^{2} E_{0}^{2}}-i \phi_{i j}(T, L)-f_{i j}(T, L)\right),
$$

with the phase given by

$$
\phi_{i j}(T, L)=\left(E_{i}-E_{j}\right) T-\left(p_{i}-p_{j}\right) L-\frac{T}{T^{d i s p}} \frac{1}{1+\left(T / T^{d i s p}\right)^{2}} \frac{\left(v_{i} T-L\right)^{2}-\left(v_{j} T-L\right)^{2}}{4 \sigma_{x e f f}^{2}},
$$

and the function $f_{i j}(T, L)$ given by

$$
f_{i j}(T, L)=\frac{1}{1+\left(T / T^{\text {disp }}\right)^{2}} \frac{\left(v_{i} T-L\right)^{2}+\left(v_{j} T-L\right)^{2}}{4 \sigma_{x e f f}^{2}} .
$$


The prefactor $N^{2}$ absorbs numerical constants.

The time integral in Eq. (96) can be evaluated with Laplace's method. We want to compute the transition probability to order $\epsilon^{2}$ in the real part of argument of the exponential, and to order $\epsilon$ in the phase. This again implies computing the minimum $T_{i j}$ of $f_{i j}(T, L)$ to order $\epsilon$, $f_{i j}\left(T_{i j}, L\right)$ to order $\epsilon^{2}$, its second derivative to order $\epsilon^{0}$, and the first derivative of the phase to order $\epsilon$. The minimum of $f_{i j}(T, L)$ is reached to order $\epsilon^{0}$ for $T=L / v_{0}$, and to order $\epsilon$ for

$$
T_{i j}=\frac{L}{v_{0}}\left(1-\frac{v_{i}+v_{j}-2 v_{0}}{2 v_{0}}\right)+\mathcal{O}\left(\epsilon^{2}\right),
$$

If the expansion (117) is used, the minimum of $f_{i j}(T, L)$ reads

$$
\begin{aligned}
f_{i j}\left(T_{i j}, L\right) & =\frac{L^{2}}{1+\ell^{2}} \frac{\left(v_{i}-v_{j}\right)^{2}}{8 v_{0}^{2} \sigma_{x e f f}^{2}} \\
& =\frac{E_{0}^{4}}{8 \sigma_{\text {peff }}^{2} \tilde{m}_{0}^{4}} \frac{\ell^{2}}{1+\ell^{2}}\left(\rho\left(1-\tilde{v}_{0}^{2}\right)-1\right)^{2}\left(\frac{\delta m_{i j}^{2}}{2 p_{0}}\right)^{2}+\mathcal{O}\left(\epsilon^{3}\right),
\end{aligned}
$$

where $\ell=L /\left(v_{0} T^{\text {disp }}\right)$ and $1-\tilde{v}_{0}^{2}=\tilde{m}_{0}^{2} / E_{0}^{2}$. The velocity $v_{0}$ has been replaced by $\tilde{v}_{0}$ in $v_{i, j}$ because $\left|\rho\left(v_{0}^{2}-\tilde{v}_{0}^{2}\right)\right| \ll 1$. The value of the second derivative of $f_{i j}(T, L)$ reads

$$
\frac{1}{2} \frac{d^{2} f_{i j}}{d T^{2}}\left(T_{i j}, L\right)=\frac{2 v_{0}^{2} \sigma_{p e f f}^{2}}{1+\ell^{2}}+\mathcal{O}(\epsilon)
$$

The value of the phase at $T=T_{i j}$ is

$$
\phi_{i j}\left(T_{i j}, L\right)=\frac{\delta m_{i j}^{2}}{2 p_{0}} L+\mathcal{O}\left(\epsilon^{2}\right) .
$$

The derivative of the phase is given to order $\epsilon$ by

$$
\begin{aligned}
\frac{d \phi_{i j}}{d T}\left(T_{i j}, L\right) & =E_{i}-E_{j}-\frac{2 \ell}{1+\ell^{2}} \sigma_{p e f f}^{2}\left(v_{i}-v_{j}\right) L \\
& =\frac{\rho\left(1-\tilde{v}_{0}^{2}\right)+\ell^{2}}{1+\ell^{2}} \frac{E_{0} \delta m_{i j}^{2}}{2 \tilde{m}_{0}^{2}}+\mathcal{O}\left(\epsilon^{2}\right),
\end{aligned}
$$

where the expansions (116)-(117) have been used. As above, the velocity $v_{0}$ has been replaced by $\tilde{v}_{0}$ in $v_{i, j}$ because $\left|\rho\left(v_{0}^{2}-\tilde{v}_{0}^{2}\right)\right| \ll 1$. The second derivative of the phase is of order $\epsilon$ and thus does not contribute to the transition probability to order $\epsilon^{2}$, at least in the argument of the exponential.

The approximation of the time average integration by Laplace's method can now be done and yields

$$
\begin{aligned}
\int d T \mathcal{A}_{i} & \mathcal{A}_{j}^{*}=v_{0} N^{2} \sigma_{p e f f} \frac{g^{2}(\mathbf{l})}{L^{2}} \\
& \times \exp \left(-2 \pi i \frac{L}{L_{i j}^{o s c}}-\frac{\left(\delta m_{i}^{2}\right)^{2}+\left(\delta m_{j}^{2}\right)^{2}}{16 \sigma_{m}^{2} E_{0}^{2}}-2 \pi^{2}\left(\frac{\rho \sigma_{x e f f}}{L_{i j}^{o s c}}\right)^{2}-\left(\frac{L}{L_{i j}^{c o h}}\right)^{2}\right)
\end{aligned}
$$


where $N^{2}$ absorbs numerical constants. The oscillation length $L_{i j}^{o s c}$ for the masses $m_{i}$ and $m_{j}$ is given by

$$
L_{i j}^{o s c}=\frac{4 \pi p_{0}}{\delta m_{i j}^{2}} .
$$

Without loss of generality, $\delta m_{i j}^{2}$ is taken to be positive. The coherence length $L_{i j}^{c o h}$ is defined by

$$
L_{i j}^{c o h}=\frac{1}{\sqrt{2} \pi} \frac{p_{0}}{\sigma_{p e f f}} L_{i j}^{o s c} .
$$

It is assumed that the time interval $\Delta T$ used to average is larger than the width of the overlap function:

$$
v_{0} \Delta T \gtrsim \sigma_{x e f f} \quad(\ell \ll 1) .
$$

Moreover, the time separation $T^{\text {sep }}$ between the wave packet peaks is supposed to be smaller than the time interval $\Delta T$. This assumption is true as long as the distance is not hugely larger than the oscillation length, since Eq. (122) shows that $T^{s e p}$ is of the order of

$$
T^{s e p}=\left|T^{i i}-T^{j j}\right| \sim \frac{L}{L_{i j}^{o s c}} \frac{1}{v_{0} p_{0}},
$$

where $1 / v_{0} p_{0}$ is of the order of a microscopic time. The violation of the condition $\Delta T \gtrsim T^{s e p}$ gives rise to the interesting possibility of detecting separate pulses, corresponding to the different mass eigenstates [176, 236]. For example, a time-dependence of the neutrino burst from the supernova SN1987A has been searched for (see [34, 234] for reviews), but only upper mass limits have been derived.

Let us now consider relativistic particles with very different masses. Without loss of generality, we suppose that $m_{i} \gg m_{j}$. As $T_{i}^{d i s p} \ll T_{j}^{d i s p}$, the approximation of taking only one dispersion time for the two mass eigenstates is not valid anymore. Therefore, we shall suppose that $L \lesssim L_{i}^{d i s p}$ and show that the decoherence sets in before the dispersion length $L_{i}^{\text {disp }}$ is reached. The calculation of the time average proceeds as in the nearly mass degenerate case, with the approximations $\ell_{i}^{2} \ll 1$ (with $\ell_{i}=L / L_{i}^{\text {disp }}$ ) and $\rho \ll \gamma_{0}^{2}$ (the particle is relativistic), so that the same result is obtained (see Eq. (127)). Now, the coherence length $L_{i j}^{\text {coh }}$ is shorter than the dispersion length $L_{i}^{d i s p}$ if $\delta m_{i j}^{2} / m_{i}^{2} \gtrsim \sigma_{p e f f} / E_{0}$. This condition is always true for very different masses, since in that case $\delta m_{i j}^{2} / m_{i}^{2} \cong 1$. Therefore, the interference becomes negligible before the dispersion of the heaviest mass eigenstate begins, so that the result (127) is also valid for very different masses.

In conclusion, the flavor-mixing transition probability (96) for a propagation distance $\mathbf{L}=$ $L \mathbf{l}$ satisfying $p_{0} / \sigma_{p}^{2} \lesssim L \lesssim \min \left(L_{i}^{\text {disp }}, L_{j}^{\text {disp }}\right)$ has the same form whatever the mass values and reads

$$
\begin{aligned}
\mathcal{P}_{\alpha \rightarrow \beta}(\mathbf{L}) & \sim v_{0} N^{2} \sigma_{p e f f} \frac{g^{2}(\mathbf{l})}{L^{2}} \sum_{i, j} V_{i \alpha} V_{\beta i}^{-1} V_{j \alpha}^{*} V_{\beta j}^{-1 *} \\
& \times \exp \left(-2 \pi i \frac{L}{L_{i j}^{o s c}}-\frac{\left(\delta m_{i}^{2}\right)^{2}+\left(\delta m_{j}^{2}\right)^{2}}{16 \sigma_{m}^{2} E_{0}^{2}}-2 \pi^{2}\left(\frac{\rho \sigma_{x e f f}}{L_{i j}^{\text {osc }}}\right)^{2}-\left(\frac{L}{L_{i j}^{\text {coh }}}\right)^{2}\right)
\end{aligned}
$$




\subsection{Oscillations with longitudinal dispersion}

\subsubsection{Amplitude}

For $L \gtrsim L_{j}^{\text {disp }}$, the momentum integration in Eq. (60) is done by the method of stationary phase in all directions, yielding in the stable case (see Eq. (95) with $\gamma_{j}\left(p_{c l, j}\right)=0$ ):

$$
\mathcal{A}_{j}=\frac{N g(\mathbf{l}) \sigma_{p e f f}}{T \sqrt{1+i T / T_{j}^{d i s p}}} \exp \left(-i m_{j} \sqrt{T^{2}-L^{2}}-f_{j}\left(p_{c l, j}\right)+\sigma_{p e f f}^{2} \frac{\left(f_{j}^{\prime}\left(p_{c l, j}\right)\right)^{2}}{1+i T / T_{j}^{d i s p}}\right)
$$

where $f_{j}(p)$ and $\sigma_{\text {peff }}$ are defined by Eqs. (82) and (89), respectively. The stationary point is given by Eq. (94):

$$
p_{c l, j}=\frac{m_{j} v_{c l}}{\sqrt{1-v_{c l}^{2}}}=\frac{m_{j} L}{\sqrt{T^{2}-L^{2}}}
$$

The corresponding energy is given by

$$
E_{c l, j}=\sqrt{p_{c l, j}^{2}+m_{j}^{2}}=\frac{m_{j} T}{\sqrt{T^{2}-L^{2}}} .
$$

The value of $f_{j}\left(p_{c l, j}\right)$ is given by $f_{j}\left(p_{c l, j}\right)=f_{j P}\left(p_{c l, j}\right)+f_{j_{D}}\left(p_{c l, j}\right)$ with

$$
f_{j P, D}\left(p_{c l, j}\right)=\frac{\left(p_{c l, j}-p_{0}\right)^{2}}{4 \sigma_{p P, D}^{2}}+\frac{\left(E_{c l, j}-E_{0}-\left(p_{c l, j}-p_{0}\right) v_{P, D}\right)^{2}}{4 \sigma_{e P, D}^{2}},
$$

while the value of $f_{j}^{\prime}\left(p_{c l, j}\right)$ is given by $f_{j}^{\prime}\left(p_{c l, j}\right)=f_{j P}^{\prime}\left(p_{c l, j}\right)+f_{j D}^{\prime}\left(p_{c l, j}\right)$, with

$$
f_{j P, D}^{\prime}\left(p_{c l, j}\right)=\frac{p_{c l, j}-p_{0}}{2 \sigma_{p P, D}^{2}}+\left(v_{c l}-v_{P, D}\right) \frac{E_{c l, j}-E_{0}-\left(p_{c l, j}-p_{0}\right) v_{P, D}}{2 \sigma_{e P, D}^{2}} .
$$

\subsubsection{Probability}

As in sections 6.1.2 and 6.2.2, the last step towards the oscillation formula consists in computing the time average of the transition probability. Examination of the term $f_{j}\left(p_{c l, j}\right)$ in Eq. (133) shows that the amplitude $\mathcal{A}_{j}$ is nearly zero unless $\left|p_{c l, j}-p_{0}\right| \lesssim \sigma_{p P, D}$ (see Eq. (136)). This condition means that the interference term $\mathcal{A}_{i} \mathcal{A}_{j}^{*}$ will be negligible unless $\left|p_{c l, i}-p_{c l, j}\right| \lesssim \sigma_{p P, D}$, that is $\left|\delta m_{i j}\right| / m \lesssim \sigma_{p P, D} / p$ (with $m$ referring to $m_{i}$ or $m_{j}$ ). In other words, the interference term is negligible if the masses are not nearly degenerate. This result is in agreement with the conclusion of section 6.2.2: if the masses $m_{i}$ and $m_{j}$ are very different, decoherence sets in before the dispersion length is reached.

It is thus possible to work with only one dispersion time $T^{d i s p}=T_{i}^{d i s p} \cong T_{j}^{d i s p}$. It is understood that for noninterference terms, i.e. $\int d T \mathcal{A}_{j} \mathcal{A}_{j}^{*}$, the dispersion time $T^{\text {disp }}$ will be taken to be $T_{j}^{d i s p}$. The integral to be computed is $\int d T \mathcal{A}_{i} \mathcal{A}_{j}^{*}$, with

$$
\mathcal{A}_{i} \mathcal{A}_{j}^{*}=\frac{N^{2} g^{2}(\mathbf{l}) \sigma_{p e f f}^{2}}{T^{2} \sqrt{1+\left(T / T^{d i s p}\right)^{2}}} \exp \left(-i \tilde{\phi}_{i j}(T, L)-\tilde{f}_{i j}(T, L)\right)
$$


with the phase given by

$$
\tilde{\phi}_{i j}(T, L)=\delta m_{i j} \sqrt{T^{2}-L^{2}}+\sigma_{\text {peff }}^{2} \frac{T}{T^{\text {disp }}} \frac{\left(f_{i}^{\prime}\left(p_{c l, i}\right)\right)^{2}-\left(f_{j}^{\prime}\left(p_{c l, j}\right)\right)^{2}}{1+\left(T / T^{d i s p}\right)^{2}},
$$

and the function $\tilde{f}_{i j}(T, L)$ defined by

$$
\tilde{f}_{i j}(T, L)=f_{i}\left(p_{c l, i}\right)+f_{j}\left(p_{c l, j}\right)-\sigma_{\text {peff }}^{2} \frac{\left(f_{i}^{\prime}\left(p_{c l, i}\right)\right)^{2}+\left(f_{j}^{\prime}\left(p_{c l, j}\right)\right)^{2}}{1+\left(T / T^{d i s p}\right)^{2}} .
$$

We would like to evaluate $\int d T \mathcal{A}_{i} \mathcal{A}_{j}^{*}$ by Laplace's method, in the same way as in section 6.2.2. This involves expanding the argument of the exponential (137) in powers of small mass differences. However, we should take care not to expand $m_{j}$ around $m_{0}$, as there is no guarantee, in the relativistic case, that $\delta m_{j}=m_{j}-m_{0}$ is much smaller than $m_{0}$. This is linked to the extreme sensitivity on $T$ of the factor $\sqrt{T^{2}-L^{2}}$ in the relativistic case. Thus the masses $m_{i}$ and $m_{j}$ should be expanded around $\tilde{m}_{0}=\left(m_{i}+m_{j}\right) / 2$. A new expansion parameter is defined by $\delta \tilde{m}_{j}=m_{j}-\tilde{m}_{0}$. The parameters $\delta \tilde{m}_{j}$ are said to be of order $\epsilon$.

Unfortunately, the value $T=\tilde{T}_{0}$ minimizing $\tilde{f}_{i j}(T, L)$ to order $\epsilon^{0}$ cannot be computed exactly. An approximate solution would be any $T$ satisfying approximately $p_{c l, j} \cong p_{0}$ and the exact solution can be computed by perturbation around it. If $T=\tilde{E}_{0} L / p_{0}$ is chosen as the approximate solution, with $\tilde{E}_{0}=\sqrt{p_{0}^{2}+\tilde{m}_{0}^{2}}$ (this solution satisfies $p_{c l, j}=p_{0}$ to order $\epsilon^{0}$ ), the value of $\tilde{T}_{0}$, to order $\delta \tilde{m}_{0}^{2}=\tilde{m}_{0}^{2}-m_{0}^{2}$, reads

$$
\tilde{T}_{0}=\frac{\tilde{E}_{0} L}{p_{0}}+\frac{\tilde{m}_{0}^{2} \delta \tilde{m}_{0}^{2}}{2 p_{0}^{3} E_{0}}(1-\rho) L,
$$

where $\rho$ is defined by Eq. (115). The expansions in $\delta \tilde{m}_{0}^{2}$ around $m_{0}$ and in $\delta \tilde{m}_{j}$ around $\tilde{m}_{0}$ will be performed to the same order. It can be checked that the value of $T$ minimizing $\tilde{f}_{i j}(T, L)$ to order $\epsilon$ is still given by $T=\tilde{T}_{0}$ (the reason is that $\tilde{m}_{0}$ is the average of $m_{i}$ and $m_{j}$ ).

The value of $\tilde{f}_{i j}(T, L)$ at its minimum reads

$$
\tilde{f}_{i j}\left(\tilde{T}_{0}, L\right)=\frac{\tilde{m}_{0}^{2}\left(\delta m_{i j}\right)^{2}+\left(\delta \tilde{m}_{0}^{2}\right)^{2}}{8 \sigma_{m}^{2} E_{0}^{2}}+\frac{E_{0}^{4}}{8 \tilde{m}_{0}^{4} \sigma_{p e f f}^{2}} \frac{\ell^{2}}{1+\ell^{2}}\left(\rho\left(1-\tilde{v}_{0}^{2}\right)-1\right)^{2}\left(\frac{\tilde{m}_{0} \delta m_{i j}}{p_{0}}\right)^{2}+\mathcal{O}\left(\epsilon^{3}\right),
$$

where $\ell=L /\left(v_{0} T^{\text {disp }}\right)$ as before, $\sigma_{m}$ is defined by Eq. (119) and $\tilde{v}_{0}=p_{0} / \tilde{E}_{0}$.

The value of the second derivative of $\tilde{f}_{i j}(T, L)$ with respect to $T$ reads

$$
\frac{1}{2} \frac{d^{2} \tilde{f}_{i j}}{d T^{2}}\left(\tilde{T}_{i j}, L\right)=\frac{2 v_{0}^{2} \sigma_{p e f f}^{2}}{1+\ell^{2}}+\mathcal{O}(\epsilon)
$$

This equation shows that the spatial width (linked to the time width by a factor $v_{0}$ ) of the wave packet associated to the oscillating particle increases linearly with $L$ for $\ell \gg 1$ (i.e. at a distance much larger than the dispersion length):

$$
\text { spatial width }=\sigma_{x e f f} \sqrt{1+\ell^{2}} \sim \frac{\sigma_{p e f f}}{p_{0}} \frac{m_{j}^{2}}{E_{0}^{2}} L .
$$


This expression agrees with the quantum-mechanical result 176, 185, derived by observing that the relation $\delta t=\frac{m^{2}}{p^{2} E} L \delta p$, obtained from $t=\frac{L}{v}$ (with $L$ kept fixed), leads to $\delta L=\frac{m^{2}}{E^{2}} \frac{\sigma_{p}}{p} L$.

The value of the phase at $T=\tilde{T}_{0}$ is

$$
\tilde{\phi}_{i j}\left(\tilde{T}_{0}, L\right)=\frac{\tilde{m}_{0} \delta m_{i j}}{p_{0}} L=\frac{\delta m_{i j}^{2}}{2 p_{0}} L+\mathcal{O}\left(\epsilon^{2}\right),
$$

where as before $\delta m_{i j}^{2}=m_{i}^{2}-m_{j}^{2}$. The derivative of the phase is given by

$$
\frac{d \tilde{\phi}_{i j}}{d T}\left(\tilde{T}_{0}, L\right)=\frac{\rho\left(1-\tilde{v}_{0}^{2}\right)+\ell^{2}}{1+\ell^{2}} \frac{E_{0} \delta m_{i j}}{\tilde{m}_{0}}+\mathcal{O}\left(\epsilon^{2}\right) .
$$

The second derivative of the phase is of order $\left(\delta \tilde{m}_{j}\right)^{2}$ and can thus be neglected. Eqs. (139), (140), (142) and (143) can be compared with Eqs. (123), (124), (125) and (126). The relation $\delta m_{i j}=\delta m_{i j}^{2} / 2 \tilde{m}_{0}$, valid for nearly degenerate masses, may be used. From that comparison, it is clear that the final result will be the same as Eq. (132).

In conclusion, the flavor-mixing transition probability (96) for a propagation distance $\mathbf{L}=$ $L 1$ satisfying $L \gtrsim L^{\text {disp }}$ reads

$$
\begin{aligned}
\mathcal{P}_{\alpha \rightarrow \beta}(\mathbf{L}) & \sim v_{0} N^{2} \sigma_{p e f f} \frac{g^{2}(\mathbf{l})}{L^{2}} \sum_{i, j} V_{i \alpha} V_{\beta i}^{-1} V_{j \alpha}^{*} V_{\beta j}^{-1 *} \\
& \times \exp \left(-2 \pi i \frac{L}{L_{i j}^{\text {osc }}}-\frac{\left(\delta m_{i}^{2}\right)^{2}+\left(\delta m_{j}^{2}\right)^{2}}{16 \sigma_{m}^{2} E_{0}^{2}}-2 \pi^{2}\left(\frac{\rho \sigma_{x e f f}}{L_{i j}^{\text {osc }}}\right)^{2}-\left(\frac{L}{L_{i j}^{\text {coh }}}\right)^{2}\right),
\end{aligned}
$$

where the relation $2 \tilde{m}_{0}^{2}\left(\delta m_{i j}\right)^{2}+2\left(\delta \tilde{m}_{0}^{2}\right)^{2}=\left(\delta m_{i}^{2}\right)^{2}+\left(\delta m_{j}^{2}\right)^{2}$, valid for nearly degenerate masses, has been used, and with $L_{i j}^{o s c}, L_{i j}^{c o h}$ defined respectively by Eqs. (128) and (129). Recall that we can set $L_{i}^{\text {disp }} \cong L_{j}^{\text {disp }}=L^{\text {disp }}$ in this regime, since decoherence occurs in the transversaldispersion regime if the mass eigenstates are not nearly degenerate.

As in section 6.2.2, the time interval $\Delta T$ used to average is assumed to be large enough. At first sight, we should have $v_{0} \Delta T \gtrsim \ell \sigma_{x e f f}$ in the limit $\ell \gg 1$ but this condition is always violated at some large distance. Actually, it is sufficient to suppose that $\Delta T \gtrsim \frac{T^{o s c}}{\gamma^{2}}\left(\gamma=\frac{E_{0}}{\tilde{m}_{0}}\right.$ is the usual Lorentz factor) so that the phase can freely oscillate around its average value (see Eq. (143) in the limit $\ell \gg 1$ ). This last condition is easily satisfied. Putting together this condition with the conditions on $\Delta T$ valid for the no-dispersion and transversal-dispersion regime (Eqs. (105) and (130)), we obtain (with $\tilde{\sigma}_{x e f f} \sim \sigma_{x e f f}$ ):

$$
\Delta T \gtrsim \max \left(\frac{\sigma_{x e f f}}{v_{0}}, \frac{T_{i j}^{o s c}}{\gamma^{2}}\right) .
$$

The relative strength of the constraints present in Eq. (145) is linked to the relative values of the dispersion and coherence lengths:

$$
\frac{\sigma_{x e f f}}{v_{0}} \gtrless \frac{T_{i j}^{o s c}}{\gamma^{2}} \quad \leftrightarrow \quad L_{i j}^{d i s p} \gtrless L_{i j}^{c o h} .
$$

It is striking that two different methods of approximation, a priori valid in different regimes, give the same oscillation formula, Eqs. (132) and (144). The dispersion length $L^{\text {disp }}$ does not 
play any special role in the final result. Each method is thus accurate enough to be extended to the whole range of distances. However, it will be seen in section 7 that the physical interpretation depends on the relative values of $L$ and $L_{i, j}^{\text {disp }}$.

\subsection{A shortcut}

We now explain another method to derive the oscillation formula (in the transversal- and longitudinal dispersion regime) which does not require the notion of a dispersion length. Although this method is shorter, it is not obvious how to apply it to unstable oscillating particles. Moreover it does not make the wave packet picture clearly apparent.

Returning to Eq. (35), we can choose to integrate on the 3-momentum before doing the energy integral. In particular, the integration on the 3-momentum can be done with the help of the Grimus-Stockinger theorem [150]. Let $\psi(\mathbf{p})$ be a 3 times continuously differentiable function on $\mathbf{R}^{3}$ such that $\psi$ itself and all its first and second derivatives decrease at least like $1 / \mathbf{p}^{2}$ for $|\mathbf{p}| \rightarrow \infty$. Then, for a real number $A>0$,

$$
\int d^{3} p \frac{\psi(\mathbf{p}) e^{i \mathbf{p} \cdot \mathbf{L}}}{A-\mathbf{p}^{2}+i \epsilon} \stackrel{L \rightarrow \infty}{\longrightarrow}-\frac{2 \pi^{2}}{L} \psi(\sqrt{A} \mathbf{l}) e^{i \sqrt{A} L}+\mathcal{O}\left(L^{-3 / 2}\right),
$$

where $L=|\mathbf{L}|$ and $\mathbf{l}=\mathbf{L} / L$. For $A<0$, the integral decreases like $L^{-2}$.

The remaining energy integral in the amplitude (35) can be done by a saddle-point approximation [144]. However, it is quicker to perform first the time average in the probability (96), which yields a delta function, and makes one of the energy integrations trivial:

$$
\int d T \mathcal{A}_{i} \mathcal{A}_{j}^{*}=\frac{N^{2}}{L^{2}} \int d E \psi\left(E, q_{i} \mathbf{l}\right) \psi^{*}\left(E, q_{j} \mathbf{l}\right) e^{i\left(q_{i}-q_{j}\right) L}
$$

where $\psi(E, \mathbf{p})$ is the overlap function defined by Eqs. (53)-(55) and $q_{j}=\sqrt{E^{2}-m_{j}^{2}} \cdot N^{2}$ absorbs numerical constants. Actually the time interval of integration $\Delta T$ is finite, so that the the delta function is only an approximation certainly valid for $\Delta T \gtrsim T_{i j}^{o s c}$.

Eq. (146) shows that the transition probability can be interpreted as an incoherent sum (i.e. occurring in the probability) over energy eigenstates: interference occurs only between the components of $\psi(E, \mathbf{p})$ having the same energy [249]. In this way, the correspondence between models with and without stationary boundary conditions is obvious: the time-integrated nonstationary probability is equivalent to the energy-integrated stationary probability. For example, the oscillation formula obtained by Grimus and Stockinger with stationary boundary conditions [150] has the form of the integrand in the right-hand side of Eq. (146). This equivalence confirms that the stationary case can be obtained from the more general nonstationary case in the limit of a vanishing energy width. Note however that the stationary limit cannot be realized in experiments and that the oscillation formula is always averaged over the energy spectrum.

In section 5.2, we have shown how a wave packet can be associated with the amplitude $\mathcal{A}_{i}$, so that oscillations can be seen, like in the quantum-mechanical treatment, as the result

\footnotetext{
${ }^{14}$ As seen from Eq. (145), this condition can be weakened to $\Delta T \gtrsim \max \left(v_{0}^{-1} \sigma_{x e f f}, \gamma^{-2} T_{i j}^{o s c}\right)$.
} 
of an interference between propagating wave packets. Thus the equivalence (146) shows that this physical picture still holds in the case of stationary boundary conditions, provided that an incoherent sum over the energy is performed, contrary to what was claimed in Ref. [151]. Therefore a wave packet picture can always be associated with the oscillation formula (once the incoherent energy average has been done), though this physical picture is well hidden in the formalism using stationary boundary conditions.

This equivalence between the time-integrated nonstationary probability and the energyintegrated stationary probability is similar to the equivalence we have met, in quantum-mechanical models, between intermediate wave packet models and stationary approaches (see Eq. (19)). However, the question of the equality of propagation times does not crop up in the quantum field theory formalism. Recall that integrations over microscopic space-time variables are included in the overlap function, with the result that the phase depends only on the average time $T$ and $L$.

Note that the time average on the probability in sections 6.2 and 6.3 could also have been done before the longitudinal-momentum integration in the amplitude, apparently yielding a delta function which makes one of the momentum integrals trivial. However this method is spoilt in 3 dimensions by the prefactor $|T-i \mu|^{-2}$. Instead of a delta function, one obtains a delta function look-alike of width $\mu^{-1} \sim \sigma_{p}^{2} / E_{0}$, introducing an additional momentum uncertainty which is larger than the mass difference $\delta m^{2} / E$ since $L_{i j}^{o s c} \gtrsim p_{0} / \sigma_{p}^{2}$. For this reason, it was preferable to avoid this shortcut in sections 6.2 and 6.3 (though it yields the same final answer as given by the following method). Moreover, it was interesting for the physical interpretation to postpone the time average, so as to obtain the explicit dependence of the amplitude on time and distance as shown in Eqs. (113) and (133).

If the coordinate system is chosen so that $\mathbf{L}$ is oriented along a coordinate axis, it is easy to rewrite the integral (146) as

$$
\int d T \mathcal{A}_{i} \mathcal{A}_{j}^{*}=\frac{N^{2} g^{2}(\mathbf{l})}{L^{2}} \int d E e^{i\left(q_{i}-q_{j}\right) L-f_{i}(E)-f_{j}(E)},
$$

with the definitions $f_{j}(E)=f_{j P}(E)+f_{j D}(E)$ and

$$
f_{j P}(E)=\frac{\left(\sqrt{E^{2}-m_{j}^{2}}-p_{0}\right)^{2}}{4 \sigma_{p P}^{2}}+\frac{\left(E-E_{0}-\left(\sqrt{E^{2}-m_{j}^{2}}-p_{0}\right) v_{P}\right)^{2}}{4 \sigma_{e P}^{2}},
$$

where $v_{P}$ and $p_{0}$ are the components of $\mathbf{v}_{P}$ and $\mathbf{p}_{0}$ along $\mathbf{L}$, while $E_{0}$ has been redefined so as to absorb the transversal part of $\mathbf{p}_{0} \cdot \mathbf{v}_{P}$. The definition of $f_{j_{D}}(E)$ is similar. The geometrical constraint $g(\mathbf{l})$ is defined by Eq. (85).

Since the phase in Eq. (147) has no stationary point, there is no problem in using Laplace's method to integrate over the energy $E$. The integrand is maximal for

$$
E_{i j}=E_{0}+\rho \frac{\delta m_{i}^{2}+\delta m_{j}^{2}}{4 E_{0}}+\mathcal{O}\left(\epsilon^{2}\right),
$$

where the dimensionless number $\rho$ is defined by Eq. (115), and $\epsilon \sim \delta m_{i}^{2} \sim \delta m_{j}^{2}$. 
The value of $f_{i}(E)+f_{j}(E)$ at the extremum reads

$$
f_{i}\left(E_{i j}\right)+f_{j}\left(E_{i j}\right)=\frac{\left(\delta m_{i}^{2}\right)^{2}+\left(\delta m_{j}^{2}\right)^{2}}{16 \sigma_{m}^{2} E_{0}^{2}}+2 \pi^{2}\left(\frac{\rho \sigma_{x e f f}}{L_{i j}^{o s c}}\right)^{2}+\mathcal{O}\left(\epsilon^{3}\right),
$$

where $\sigma_{x e f f}, \sigma_{m}$ and $L_{i j}^{o s c}$ are defined by Eqs. (89), (119) and (128), respectively. The value of the second derivative of $f_{i}(E)+f_{j}(E)$ at the extremum reads

$$
\frac{1}{2} \frac{d^{2}\left(f_{i}+f_{j}\right)}{d E^{2}}\left(E_{i j}\right)=\frac{1}{2 v_{0}^{2} \sigma_{p e f f}^{2}}+\mathcal{O}(\epsilon) .
$$

The expansion of the phase around the extremum reads

$$
\phi_{i j}(E) \cong-\frac{\delta m_{i j}^{2}}{2 p_{0}} L+\frac{\delta m_{i j}^{2}}{2 p_{0}^{2} v_{0}} L\left(E-E_{i j}\right) .
$$

The second derivative of the phase is of order $\epsilon$ and can be neglected with respect to the second derivative of $f_{i}+f_{j}$. The approximation of the integral (147) by Laplace's method is now straightforward and yields

$$
\begin{aligned}
\int d T \mathcal{A}_{i} \mathcal{A}_{j}^{*}=v_{0} N^{2} \sigma_{p e f f} \frac{g^{2}(\mathbf{l})}{L^{2}} \\
\quad \times \exp \left(-2 \pi i \frac{L}{L_{i j}^{o s c}}-\frac{\left(\delta m_{i}^{2}\right)^{2}+\left(\delta m_{j}^{2}\right)^{2}}{16 \sigma_{m}^{2} E_{0}^{2}}-2 \pi^{2}\left(\frac{\rho \sigma_{x e f f}}{L_{i j}^{o s c}}\right)^{2}-\left(\frac{L}{L_{i j}^{c o h}}\right)^{2}\right)
\end{aligned}
$$

The linear superposition of the different partial transition probabilities $\int d T \mathcal{A}_{i} \mathcal{A}_{j}^{*}$ gives the

same result as those obtained in the transverse- and longitudinal-dispersion regimes (Eqs. (132) and (144)). Though the computation is shorter, the origin of the decoherence and localization terms is not as clear (see section 7), because 'wave packets' in configuration space do not appear explicitly at any stage of the calculation. Moreover the oscillation formula (111) valid in the no-dispersion regime cannot be exactly reproduced.

\section{Analysis of the probability in the stable case}

In section 6, the oscillation probability was computed in the three distance ranges studied in section 5, yielding the formulas (111), (132) and (144). In this section, we analyze the different terms appearing in these formulas and we explain their origin. This analysis will allow us to answer most of the questions raised in section 3 .

Before analyzing the oscillation formulas, let us sum up the assumptions used in its derivation. The oscillating particle is stable, and propagates in vacuum over a macroscopic distance $L$, i.e. $L \gg 1 / p_{0}$, where $p_{0}$ is the average momentum of the particle. The velocity of the oscillating particle is arbitrary, but the condition $\delta m_{i j}^{2} \ll E_{0}^{2}$ is assumed to be satisfied. This means that nonrelativistic particles are supposed to have nearly degenerate masses (if it were not the case, oscillations would vanish anyway). The oscillation formula has been derived for a scalar particle, 
but this assumption is not very restrictive, since the spin structure factorizes from the sum on the mass eigenstates as long as $\delta m_{i j}^{2} \ll E_{0}^{2}$.

In order to obtain a time-independent formula, the transition probability has been averaged over a time interval $\Delta T$ satisfying $\Delta T \gtrsim \max \left(v_{0}^{-1} \sigma_{x e f f}, \gamma^{-2} T_{i j}^{o s c}\right)$. We have also supposed that the time separation $T^{s e p}$ between the wave packets, given by Eq. (131), is smaller than the time interval $\Delta T$. The dispersion has been taken into account.

The oscillation formula valid at large distance (i.e. in the second and third regimes) will be studied first since it is the generic experimental case.

\subsection{Oscillations at large distance}

\subsubsection{Oscillation formula at large distance}

At large distance $\left(L \gtrsim p_{0} / \sigma_{p}^{2}\right)$, the flavor-mixing transition probability for a stable particle of arbitrary velocity propagating over a distance $\mathbf{L}=L \mathbf{l}$, with dispersion taken into account, is given in a very good approximation by Eqs. (132), (144) or (151):

$$
\begin{aligned}
\mathcal{P}_{\alpha \rightarrow \beta}(\mathbf{L}) & =\frac{N_{g} g^{2}(\mathbf{l})}{L^{2}} \sum_{i, j} V_{i \alpha} V_{\beta i}^{-1} V_{j \alpha}^{*} V_{\beta j}^{-1 *} \\
& \times \exp \left(-2 \pi i \frac{L}{L_{i j}^{o s c}}-\frac{\left(\delta m_{i}^{2}\right)^{2}+\left(\delta m_{j}^{2}\right)^{2}}{16 \sigma_{m}^{2} E_{0}^{2}}-2 \pi^{2}\left(\frac{\rho \sigma_{x e f f}}{L_{i j}^{o s c}}\right)^{2}-\left(\frac{L}{L_{i j}^{c o h}}\right)^{2}\right),
\end{aligned}
$$

where $g(\mathbf{l})$ is the geometrical factor defined by Eq. (85):

$$
g(\mathbf{l})=\exp \left(-\frac{\left(\mathbf{p}_{0} \times \mathbf{l}\right)^{2}}{4 \sigma_{p}^{2}}\right),
$$

with $\sigma_{p}$ defined by Eq. (67). Recall that the function $g(\mathbf{l})$ restricts the propagation to a cone of axis $\mathbf{p}_{0}$ and angle $\arcsin \left(\sigma_{p} / p_{0}\right)$ (see Fig. 6). $N_{g}$ is a normalization constant determined by the conservation of the probability, in the case of a stable oscillating particle:

$$
\sum_{\beta} \int L^{2} d \Omega \mathcal{P}_{\alpha \rightarrow \beta}(\mathbf{L})=1 .
$$

From Eq. (152), we can see that $\sum_{\beta} \mathcal{P}_{\alpha \rightarrow \beta}(\mathbf{L})$ is independent of $L$, since $V^{-1}=V^{\dagger}$ for stable particles (unless the indices $\alpha, \beta$ are restricted to active flavors in the presence of sterile flavors). Note that the unitarity relation is only verified to order $\epsilon$ because of the approximations made in the computations. Thus the normalization constant $N_{g}$ is fixed by

$$
N_{g} \int d \Omega g^{2}(\mathbf{l})=1
$$

The question of the normalization of the probability will be addressed in more detail in section 9.1. The oscillation and coherence lengths are defined by Eqs. (128) and (129), respectively:

$$
L_{i j}^{o s c}=\frac{4 \pi p_{0}}{\delta m_{i j}^{2}} \quad \text { and } \quad L_{i j}^{c o h}=\frac{1}{\sqrt{2} \pi} \frac{p_{0}}{\sigma_{p e f f}} L_{i j}^{o s c} .
$$




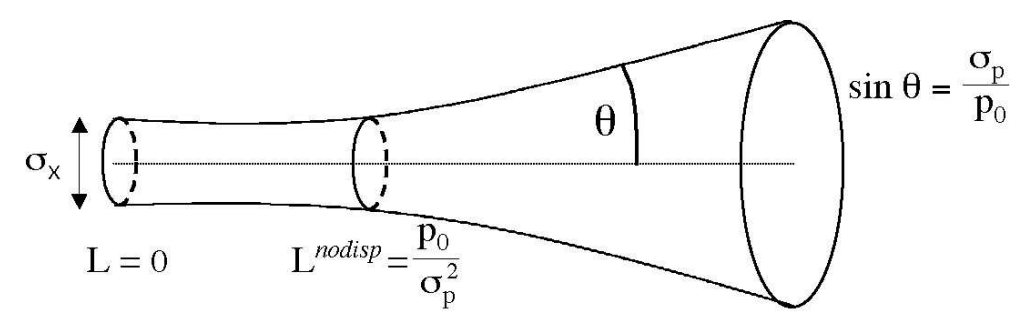

Figure 6: The probability of detection is maximal within a cone at large distance and within a cylinder at short distance.

The effective width $\sigma_{x e f f}$ is defined by $\sigma_{p e f f} \sigma_{x e f f}=1 / 2$, with $\sigma_{p e f f}$ given by Eq. (89):

$$
\frac{1}{\sigma_{p e f f}^{2}}=\frac{1}{\sigma_{p P}^{2}}+\frac{1}{\sigma_{p D}^{2}}+\frac{\left(v_{0}-v_{P}\right)^{2}}{\sigma_{e P}^{2}}+\frac{\left(v_{0}-v_{D}\right)^{2}}{\sigma_{e D}^{2}}
$$

The dimensionless parameter $\rho$ is defined by Eq. (115):

$$
\rho=\sigma_{p e f f}^{2}\left(\frac{1}{\sigma_{p P}^{2}}+\frac{1}{\sigma_{p D}^{2}}-\frac{v_{P}\left(v_{0}-v_{P}\right)}{\sigma_{e P}^{2}}-\frac{v_{D}\left(v_{0}-v_{D}\right)}{\sigma_{e D}^{2}}\right) .
$$

The mass width $\sigma_{m}$ is defined by Eq. (119):

$$
\frac{1}{\sigma_{m}^{2}}=\sigma_{p e f f}^{2}\left(\frac{1}{\sigma_{p}^{2}}\left(\frac{1}{\sigma_{e P}^{2}}+\frac{1}{\sigma_{e D}^{2}}\right)+\frac{\left(v_{P}-v_{D}\right)^{2}}{\sigma_{e P}^{2} \sigma_{e D}^{2}}\right) .
$$

As will be made clear in the following sections, the oscillation formula (152) reduces to the standard formulas (12) and (14) (with the additional property of $1 / L^{2}$ geometrical decrease) if the observability conditions $\left|\mathbf{p}_{0} \times \mathbf{l}\right| \lesssim \sigma_{p}, L \ll L_{i j}^{c o h}$ and $L_{i j}^{o s c} \gg S_{P, D}$ are satisfied.

\subsubsection{Oscillation phase}

Each term of the sum appearing in Eq. (152) oscillates with a phase given by $2 \pi L / L_{i j}^{\text {osc }}$. This phase is independent of the details of production and detection up to order $\epsilon$ (except the dependence on the momentum $p_{0}$, of course). Its origin can be better understood by going back to the expression of the probability before the time average is done.

1. In the transversal-dispersion regime, the phase is given by Eq. (120). In the limit $T \ll$ $T^{\text {disp }}$, the third term of $(120)$ can be neglected and the phase reads

$$
\phi_{i j}(T, L) \cong\left(E_{i}-E_{j}\right) T-\left(p_{i}-p_{j}\right) L .
$$

Using the expansions (114) and (116), we can write

$$
E_{j} T-p_{j} L \cong E_{0} T-p_{0} L+\frac{\delta m_{j}^{2}}{2 p_{0}}\left(L+\rho\left(v_{0} T-L\right)\right) .
$$


The explicit expression for $\rho$ is not useful, as we shall see below. The oscillating phase $\phi_{i j}(T, L)$ can now be written

$$
\phi_{i j}(T, L) \cong 2 \pi \frac{L}{L_{i j}^{o s c}}+2 \pi \rho \frac{v_{0} T-L}{L_{i j}^{o s c}}
$$

Since $\left|v_{0} T-L\right| \lesssim \sigma_{x e f f}$ (see Eq. (113)), the second term of the right-hand side of Eq. (159) is negligible if

$$
|\rho| \frac{\sigma_{x e f f}}{L_{i j}^{o s c}} \ll 1
$$

Such a constraint is indeed present in the third exponential term of the probability (152), so that either the second term of the right-hand side of Eq. (159) does not contribute to the phase, or the corresponding interference term in the probability is negligible.

Since the leading term of the oscillating phase does not depend on $\rho$, the phase is independent to order $\epsilon$ of the exact values of $E_{i, j}$ and $p_{i, j}$. In other words the phase is independent of the conditions of production and detection. The two fundamental reasons for this independence are clearly seen in Eq. (158):

(a) the particle is on-shell: $E_{j}=\sqrt{p_{j}^{2}+m_{j}^{2}}$;

(b) the particle is well localized in space-time: $\left|v_{0} T-L\right| \lesssim \sigma_{x e f f}$.

2. In the longitudinal-dispersion regime, the phase is given by Eq. (138). In the limit $T \gg$ $T^{d i s p}$, the second term of the right-hand side of Eq. (138) can be neglected. The phase now reads

$$
\tilde{\phi}_{i j}(T, L) \cong \delta m_{i j} \sqrt{T^{2}-L^{2}}
$$

Using the expansions (142) and (143) around the average propagation time $\tilde{T}_{0}$, the phase can be written in the limit $\ell \gg 1$ as

$$
\tilde{\phi}_{i j}(T, L) \cong 2 \pi \frac{L}{L_{i j}^{o s c}}+\frac{E_{0} \delta m_{i j}}{\tilde{m}_{0}}\left(T-\tilde{T}_{0}\right) .
$$

The second term of this equation is small if the spatial spread of the wave packet is smaller than $L_{i j}^{o s c} / \gamma^{2}$, where $\gamma$ is the usual Lorentz factor. Since the possible time range is constrained by the width of the overlap function, i.e. $\left|T-\tilde{T}_{0}\right| \lesssim \ell \sigma_{x e f f} / v_{0}$ (see Eq. (140)), the second term of the right-hand side of Eq. (161) is negligible if

$$
\frac{\sigma_{p e f f}}{p_{0}} \frac{L}{L_{i j}^{o s c}} \ll 1
$$

i.e. if $L \ll L_{i j}^{c o h}$. Such a constraint is indeed present in the last exponential term of the probability (152). If it is satisfied, the oscillating phase is equal to $2 \pi L / L_{i j}^{o s c}$, as usual. If not, the $i j$ interference term vanishes and decoherence occurs. 


\subsubsection{Coherence length}

The last exponential term in the probability (152) shows that the $i j$-interference term vanishes at a distance larger than the coherence length $L_{i j}^{c o h}$. This decoherence, predicted by Nussinov [216], has two possible origins, since both the overlap function and the derivative of the phase contribute to $\exp \left(-\left(L / L_{i j}^{c o h}\right)^{2}\right)$. The physical explanation depends on whether the coherence length is larger than the dispersion length or not't

1. If the coherence length is smaller than the dispersion length (here $L^{d i s p}=\min \left(L_{i}^{\text {disp }}, L_{j}^{d i s p}\right)$ ), decoherence takes place in the transversal-dispersion regime. In that case, the decoherence term comes mainly from the function $f_{i j}\left(T_{i j}, L\right)$, i.e. from the overlap function. This is clear from Eq. (123), with the dispersion neglected for simplicity: $\ell \ll 1$. This decoherence arises from the progressive separation of the wave packets, due to the different group velocities $v_{i}$ and $v_{j}$ : if $\mathrm{T}$ is large enough, both terms $\left|v_{i} T-L\right|$ and $\left|v_{j} T-L\right|$ cannot remain small.

2. If the coherence length is larger than the dispersion length (here $L^{\text {disp }} \cong L_{i}^{\text {disp }} \cong L_{j}^{\text {disp }}$ ), decoherence takes place in the longitudinal-dispersion regime. In that case, the decoherence term does not come anymore from the separation of the wave packets since the packets spread out beyond the dispersion length as quickly as they separate (see Eq. (141)). Still, decoherence arises from the variation of the phase over the width of the overlap function (see Eq. (161)). The interference term is averaged to zero by the time integral when the spatial spread of the 'wave packet' becomes larger than $L_{i j}^{o s c} / \gamma^{2}$. A similar mechanism has been observed in connection with neutron interferometry [186].

All in all, the coherence length arises not only from the separation of wave packets, as it is usually explained in the literature: in the case of nearly degenerate masses, it can also originate in a too large dispersion of the wave packet in comparison with the oscillation length (Fig. 7).

Remark that the phenomena of separation of wave packets and dispersion contribute to the coherence length in both regimes: if approximations such as $\ell \ll 1$ (or $\ell \gg 1$ ) are not made, terms proportional to $L^{2}$ come from $f_{i j}\left(T_{i j}, L\right)$ (or $\tilde{f}_{i j}\left(\tilde{T}_{0}, L\right)$ ) and from the squared derivative of the phase, divided by the second derivative of $f_{i j}$ (or of $\tilde{f}_{i j}$ ). Furthermore, the transition between the two regimes is not visible in the oscillation formula, because the two decoherence mechanisms smoothly blend.

Besides the above explanations in configuration space, the coherence length can be also understood in energy-momentum space, where it is due to the variation of the phase over the 'wave packet' width $\sigma_{p e f f}$ (see Eq. (150)). The explanation in momentum space is thus simpler, but the two mechanisms at the origin of the coherence length in configuration space are more intuitive.

\footnotetext{
${ }^{15}$ As noted in section 6.2.2, the condition $L^{\text {coh }} \gtrsim L^{d i s p}$ can be written as $\frac{\delta m^{2}}{m^{2}} \lesssim \frac{v_{0}^{2} \sigma_{p}}{p_{0}}$, which is equivalent to say that the masses are nearly degenerate.
} 


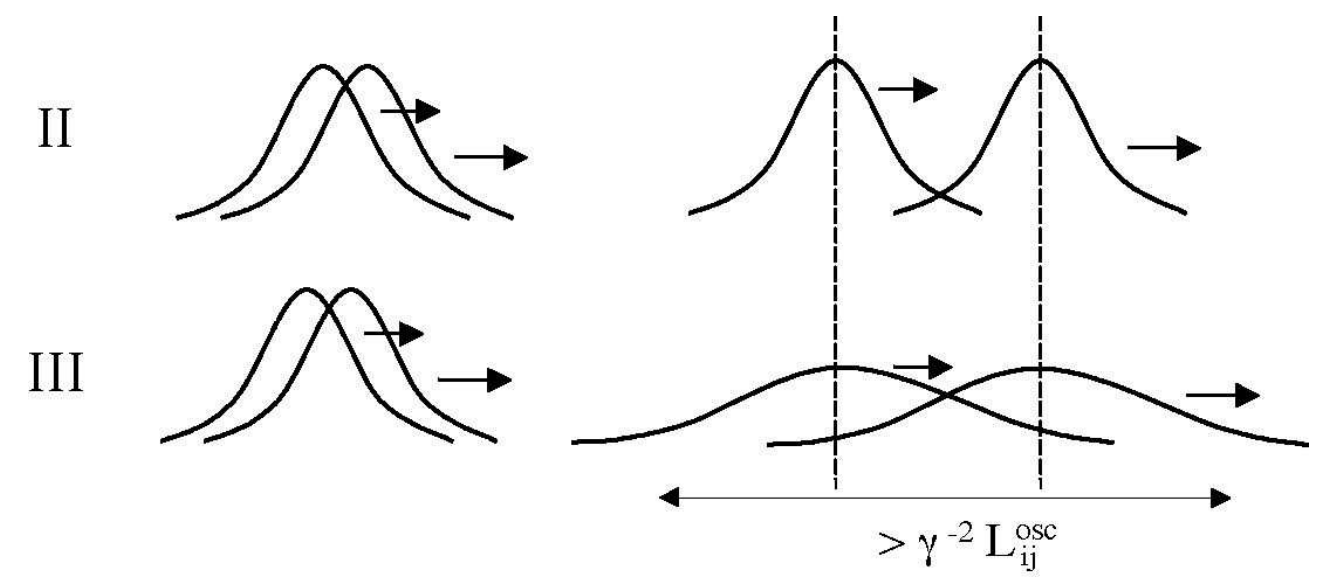

Figure 7: In the transversal-dispersion regime (II), the coherence length is due to the non-overlap of wave packets, whereas it is due in the longitudinal-dispersion regime (III) to their dispersion (the coherence length was not computed in the no-dispersion regime).

As dispersion is usually neglected in the literature, it is interesting to derive the oscillation formula with this approximation $\left(T_{j}^{d i s p}=\infty\right)$. Such a computation in the transversal-dispersion regime yields the same result as when dispersion is taken into account (Eq. (152)), except that the following substitution has to be made:

$$
L_{i j}^{c o h} \rightarrow \frac{L_{i j}^{c o h}}{\left|\rho\left(1-v_{0}^{2}\right)-1\right|} \quad(F A L S E) .
$$

The incorrect multiplying factor has its origin in Eq. (117): in the limit $T_{j}^{d i s p}=\infty$, the coherence length term arises only from the difference between the group velocities $v_{i}$ and $v_{j}$. However the factor $\left|\rho\left(1-v_{0}^{2}\right)-1\right|$ tends to 1 in the relativistic limit, so that the substitution (163) becomes trivial in that limit. This observation explains why our result (152) coincides with Eq. (26) of Ref. [144], as the authors of this article, while neglecting dispersion, consider only relativistic neutrinos. Note however that even relativistic neutrinos spread at large distances so that a calculation neglecting dispersion such as in Ref. [144] is only valid for $L \lesssim L_{j}^{\text {disp }}$.

It is interesting to observe that the coherence length increases when a long coherent measurement in time is performed at the detector, even if the oscillating 'wave packets' have separated spatially [182, 183]. In that case, the energy uncertainty at detection goes to zero, $\sigma_{e D} \rightarrow 0$, so that the effective width also goes to zero, $\sigma_{\text {peff }} \rightarrow 0$, and the coherence length becomes infinite, $L_{i j}^{c o h} \rightarrow \infty$.

After all these theoretical considerations, it must be said that the decoherence at the level of the wave packet is irrelevant in most experiments, since it is usually dominated by decoherence effects originating in the energy spread of the beam. Different situations are discussed in section 9.2.2. Finally, let us remark that the exponential decrease in $L^{2}$ of this decoherence term is model-dependent. It results here from the Gaussian approximation. However, the definition of the coherence length is model-independent, apart from a multiplying constant. 


\subsubsection{Localization}

A third kind of term appearing in the flavor-mixing transition probability (152) are localization terms, that is, observability constraints imposing that the oscillation length should be larger than the space-time uncertainty: $L_{i j}^{o s c} \gtrsim \sigma_{x e f f}$. This condition can be rewritten as $\delta m_{i j}^{2} / p_{0} \lesssim$

$\sigma_{p e f f}$, stating that oscillations vanish if the energy-momentum measurements allow to distinguish between the different mass eigenstates.

Two localization terms appear in the probability (152), while one more is implicitly assumed when applying the Jacob-Sachs theorem:

1. The term containing $\sigma_{m}$ can be rewritten

$$
\frac{\left(\delta m_{i}^{2}\right)^{2}+\left(\delta m_{j}^{2}\right)^{2}}{16 \sigma_{m}^{2} E_{0}^{2}}=\frac{\left(\delta m_{i j}^{2}\right)^{2}}{32 \sigma_{m}^{2} E_{0}^{2}}+\frac{\left(\delta m_{i}^{2}+\delta m_{j}^{2}\right)^{2}}{32 \sigma_{m}^{2} E_{0}^{2}} .
$$

The first term of the right-hand side of Eq. (164) can be written as a localization term:

$$
L_{i j}^{o s c} \gtrsim \sigma_{x}
$$

as $\sigma_{m} \sim v_{0} \sigma_{p}$ whether the stationary limit is taken or not. The second term of the righthand side of Eq. (164) is not a localization term and will be discussed in section 7.1.5.

2. The term containing $\rho$ is also a localization term and imposes that

$$
L_{i j}^{o s c} \gtrsim|\rho| \sigma_{x e f f}
$$

It is not obvious whether this constraint is stronger than the previous one, i.e. whether it is possible that $|\rho| \sigma_{x e f f} \gg \sigma_{x}$. This situation might arise from an energy uncertainty much smaller than the momentum uncertainty. In that case, the definition (155) of the effective width shows that $\sigma_{x e f f} \gg \sigma_{x}$. Say, for example, that the energy uncertainty at the detection goes to zero, i.e. $\sigma_{e D} \rightarrow 0$. With the help of Eqs. (155), (156) and (58), we see that

$$
|\rho| \sigma_{x e f f} \stackrel{\sigma_{e D \rightarrow 0}}{\longrightarrow} \frac{\left|v_{D}\right|}{\sigma_{e D}} \lesssim S_{D}
$$

where $S_{D}$ is the size of the macroscopic detection region. Thus the localization term does not give a stronger constraint than $L_{i j}^{o s c} \gtrsim S_{D}$. This constraint is always satisfied, as it is equivalent to the constraint obtained by averaging the transition probability over the production region (see section 9.2.1). Therefore, the coherence length can be increased without bound by more accurate energy measurements, contrary to what was claimed in Refs. [144, 139]. Note that this is not true if the accuracy of the 3-momentum measurements is increased, as the localization makes the oscillations vanish when the corresponding spatial uncertainty becomes larger than the oscillation length. Of course the opposite conclusions would be reached if experiments measured time, not distance.

3. The contour integral used in the Jacob-Sachs theorem yields a third localization constraint. The poles corresponding to the mass eigenstates $m_{i}$ and $m_{j}$ cannot be both included in 


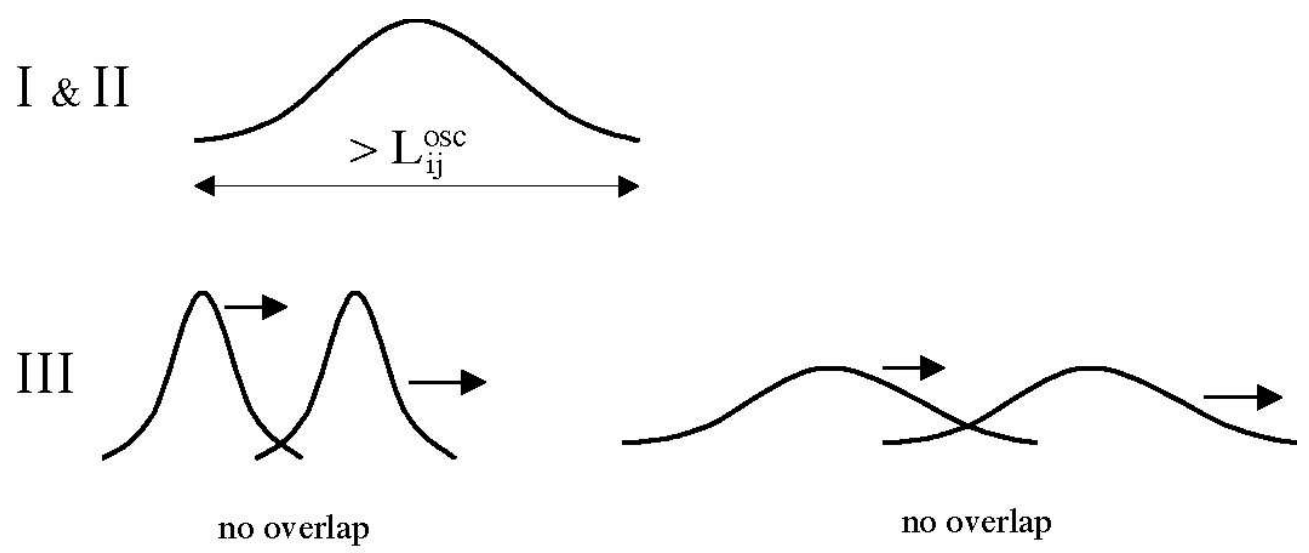

Figure 8: In the no-dispersion and transversal-dispersion regimes (I and II), the localization condition is due to the initial spread of the wave packet, whereas it is due in the longitudinaldispersion regime (III) to their non-overlap.

Table 1: Origins of the coherence length and localization terms.

\begin{tabular}{|c|c|c|}
\hline & Transversal-dispersion regime & Longitudinal-dispersion regime \\
\hline $\begin{array}{c}\text { Coherence length } \\
\text { Localization }\end{array}$ & $\begin{array}{c}\text { decreasing overlap of wave packets } \\
\text { initial spread of each wave packet }\end{array}$ & $\begin{array}{c}\text { increasing dispersion of each wave packet } \\
\text { constant overlap of wave packets }\end{array}$ \\
\hline
\end{tabular}

the same contour integration unless $\left|\delta m_{i j}^{2}\right| / p_{0} \lesssim \sigma_{p}$. This third constraint is not stronger than the previous ones and can be ignored.

As was the case for the coherence length, there are two origins for the localization term containing $\rho$ (Fig. 8).

1. In the limit $\ell \ll 1$ (transversal-dispersion regime), this term comes from the variation of the phase over the width of the wave packet (see Eq. (159)). If the oscillation length is smaller than the width of the wave packet, the interference term is averaged to zero by the time integration.

2. In the limit $\ell \gg 1$ (longitudinal-dispersion regime), this term arises from the overlap function (see Eq. (139)). Thus it comes from the separation of the wave packets, which remains constant in the longitudinal-dispersion regime.

Recall that the coherence length was shown to arise, in the transversal-dispersion regime, from the wave packet separation and, in the longitudinal-dispersion regime, from the variation of the phase. Therefore, the coherence-length and localization conditions in the transversaldispersion regime transform respectively into localization and coherence-length conditions in the longitudinal-dispersion regime. The situation is summarized in Table 1. 


\subsubsection{Energy-momentum conservation}

The last term of the oscillation probability (152) to be discussed is the second term in the decomposition of Eq. (164). This term could be a matter of concern since it does not vanish in the limit $m_{i}=m_{j}$. Note that this kind of term is not specific to our computation. For example, it would appear in the oscillation formula (26) of Ref. [144] if the terms $S_{a}\left(E_{a}\right)$ present in Eq. (22) of that article had been expanded beyond zeroth order in $m_{a}^{2} / E_{a}^{2}$.

The second term in Eq. (164) imposes that

$$
\frac{\left|m_{i}^{2}+m_{j}^{2}-2 m_{0}^{2}\right|}{E_{0}} \lesssim \sigma_{m} .
$$

As $m_{0}$ is related to the average in- and outgoing momentum by $m_{0}^{2}=E_{0}^{2}-p_{0}^{2}$, condition (168) means that the mass eigenstates have to be on-shell with respect to $\left(E_{0}, p_{0}\right)$ within the uncertainty $\sigma_{m}$. For example, this constraint is impossible to satisfy in the mixing of relativistic and nonrelativistic neutrinos if the available energy-momentum is such that only the lightest neutrino can be produced. However it has no effect on the oscillations in the two cases considered in the present report, namely relativistic particles or nearly mass degenerate particles. Condition (168) should simply be considered as expressing the conservation of energy-momentum. Such kinematical constraints are usually not included in the oscillation formula, though they rightly belong to it. A complete computation of the transition probability should not only include this energy-momentum constraint, but also similar terms arising from the interaction amplitudes $M_{P, D}$, from the prefactor resulting from the Gaussian integration and from the spin structure of the propagator. Actually the masses of the oscillating particle should be expected to appear not only through mass differences but also through their absolute values.

\subsection{Oscillations at short distance}

At short distance $\left(L \lesssim p_{0} / \sigma_{p}^{2}\right)$, the oscillation formula is given by Eq. (111):

$$
\begin{aligned}
\mathcal{P}_{\alpha \rightarrow \beta}(\mathbf{L})= & N_{\tilde{g}} \exp \left(-\frac{\left(\tilde{\mathbf{v}}_{0} \times \tilde{\mathbf{L}}\right)^{2}}{2 \sigma_{x}^{2} \tilde{\mathbf{v}}_{0}^{2}}\right) \sum_{i, j} V_{i \alpha} V_{i \beta}^{*} V_{j \alpha}^{*} V_{j \beta} \\
& \times \exp \left(-2 \pi i \frac{L}{L_{i j}^{o s c}}-\frac{\left(\delta m_{i}^{2}\right)^{2}+\left(\delta m_{j}^{2}\right)^{2}}{16 \tilde{\sigma}_{m}^{2} E_{0}^{2}}-2 \pi^{2}\left(\frac{\tilde{\rho} \tilde{\sigma}_{x e f f}}{L_{i j}^{o s c}}\right)^{2}\right),
\end{aligned}
$$

where $\tilde{\sigma}_{x e f f}$ and $L_{i j}^{o s c}$ are defined by Eq. (106) and Eq. (112), respectively. $N_{\tilde{g}}$ includes all prefactors independent of $L$ as well as normalization factors.

The normalization of the probability can be done in the same way as in Eq. (153):

$$
N_{\tilde{g}} \int L^{2} d \Omega \exp \left(-\frac{\left(\tilde{\mathbf{v}}_{0} \times \tilde{\mathbf{L}}\right)^{2}}{2 \sigma_{x}^{2} \tilde{\mathbf{v}}_{0}^{2}}\right)=1 .
$$

As in the case of oscillations at large distance, it can be argued that the two last exponential terms in Eq. (169) yield an observability condition not stronger than $L_{i j}^{o s c} \gtrsim S_{P, D}$ (see section 
7.1.4). It could also be interesting to understand the origin of the oscillation phase by going back to the amplitude, before doing the time average. This discussion can be found in Ref. (60]).

Actually, the oscillation formula (169) is very similar to the formula valid at large distance (Eq. (152)). There are three main differences. First there is no coherence-length term in Eq. (169), which is due to the neglect of the terms beyond $\mathcal{O}\left(\epsilon^{0}\right)$ in the evaluation of $F\left(T_{0}\right)$ (see Eq. (79)). Second, the geometrical decrease in $1 / L^{2}$ is lacking in Eq. (169), which is explained by the fact that the dispersion of the oscillating particle 'wave packet' is not yet significant. Finally, the directional constraint present in Eq. (169) confines the propagation to a cylinder of radius $r$ (with $\sigma_{x} \lesssim r \lesssim S_{P, D}$ ), whereas the directional constraint present in Eq. (152) restricts the oscillating particle propagation to a cone of angle $\arcsin \left(\sigma_{p} / p_{0}\right)$ (Fig. 6 in section 7.1.1). This different behavior is also a result of the absence of dispersion for $L \lesssim p_{0} / \sigma_{p}^{2}$.

In conclusion, the oscillation formula (169) reduces to the standard formulas (12) and (14) if the observability conditions $\left|\mathbf{v}_{0} \times \mathbf{L}\right| \lesssim\left|\mathbf{v}_{0}\right| \sigma_{x}$ and $L_{i j}^{o s c} \gg S_{P, D}$ are satisfied.

\subsection{Answers at last}

In section 3, our review of field-theoretical treatments brought to the fore several questions:

- Is there a limit in which the oscillation formula, derived with stationary boundary conditions, can be obtained from the oscillation formula derived with external wave packets?

- Does an infinitely precise measurement of the energy lead to an infinite coherence length, or is there decoherence anyway? This question is inseparable from the first one.

- Does a strong localization of the source lead to a dependence of oscillation formula on mass prefactors?

- Do 'plane waves oscillations' exist?

The external wave packet model used in this report is of sufficient generality to allows us to clarify and answer these questions.

\subsubsection{Existence of a stationary limit}

Two important results concerning stationary boundary conditions have been demonstrated. First, there is no contradiction between models with stationary boundary conditions and those with external wave packets. The former type of model can be obtained from the latter in a smooth limit. Let us state again the gist of the problem. Stationary boundary conditions, given by $v_{P, D}=0$ and $\sigma_{e P, D}=0$, lead to an infinite effective width $\sigma_{x e f f}$ and thus to an infinite coherence length $L_{i j}^{\text {coh }}$ (see Eqs. (154)-(155)). Hence, the localization term depending on $\rho \sigma_{x e f f} / L_{i j}^{o s c}$ seems to diverge in Eq. (152), with the result that the $i j$-interference term seems to vanish in the stationary limit $\sigma_{x e f f} \rightarrow \infty$. If it were true, it would be impossible to increase without limit the coherence length by performing long coherent measurements. This would be in contradiction with stationary boundary condition models, which have a zero energy uncertainty 
but an infinite coherence length. However the product $\rho \sigma_{x e f f}$ remains finite, as shown by Eq. (167), so that the interference term does not vanish. For example, the Grimus-Stockinger model [150] is obtained in the limit $\rho=0, \sigma_{x e f f} \rightarrow \infty$ with $\rho \sigma_{x e f f}=0$. The latter condition means that this model can be recovered from the external wave packet model if the stationary limit has the property $v_{P, D} / \sigma_{e P, D}=0$ (see discussion after Eq. (58)). The only localization condition comes from the momentum spreads of the source and detector (see Eq. (165)). Note also that the amplitude $\mathcal{A}_{j}$ (see Eq. (60) ) takes in the stationary limit the form of a plane wave, with a well-defined energy and momentum (see Eqs. (114)-(116) with $\rho \rightarrow 0$ ).

The second important result is the equivalence (146) between the time-integrated nonstationary probability and the energy-integrated stationary probability. Since oscillations can be described in the former model by interfering 'wave packets', this interpretation is also valid in the latter, contrary to what was claimed in [151]. Thus the incoherent superposition of stationary amplitudes, which have a plane wave form, reproduces the characteristics of wave packet propagation. Of course, this is not true if the probability is not integrated over the energy spectrum, but we have already argued that a source and a detector with zero energy uncertainties do not constitute realistic boundary conditions.

\subsubsection{Unbounded coherence length}

As shown by Kiers, Nussinov and Weiss [182], a more precise measurement of the energy at detection increases the coherence length. This feature is reproduced within the external wave packet model: $L_{i j}^{\text {coh }} \sim \sigma_{x e f f} \sim 1 / \sigma_{e D}$ (see Eqs. (154)-(155)). In the limit $\sigma_{e D} \rightarrow 0$, the external wave packet model becomes equivalent to a stationary model (even if the source is nonstationary), as the detector picks up wave packet's components having exactly the same energy. Contrary to what was asserted in Refs. [144, 139], oscillations do not vanish in that limit since we argued above that $\rho \sigma_{x e f f}$ remains finite.

\subsubsection{No mass prefactors at large distance}

Contrary to what was claimed by Shtanov [242], mass prefactors never appear in front of the oscillating exponentials present in the transition probability (152). Let us first explain what these 'mass prefactors' mean. Shtanov derives oscillation formulas for neutrinos within a sourcepropagator model in configuration space. Shtanov computes the convolution of the neutrino propagator with a source, keeping only the phase in the oscillating exponentials but neglecting the contributions depending on the width $\sigma_{x}$ of the source. With this last approximation, the space-time localization (i.e. the 'wave packet envelope') of the amplitude is lost.

Let us consider his model will be considered in a scalar version for an easier comparison with our results. The propagator for a scalar particle propagating from $x$ to $x^{\prime}$ is asymptotically $\left(m_{j} \sqrt{\left(x^{\prime}-x\right)^{2}} \gg 1\right)$ given in configuration space by

$$
\mathcal{A}_{j} \sim \frac{\sqrt{m_{j}}}{\left(\left(x^{\prime}-x\right)^{2}\right)^{3 / 4}} e^{-i m_{j} \sqrt{\left(x^{\prime}-x\right)^{2}}},
$$


where $\left(x^{\prime}-x\right)^{2}$ is the Lorentz interval. The convolution of Eq. (171) with a monochromatic source yields without problem the standard oscillation formula (12). However, convolution with a strongly localized source $\left(\sigma_{x, t} \lesssim 1 / E\right)$ leads to an amplitude that keeps its dependence on the mass prefactor $\sqrt{m_{j}}$. In that case, the transition probability is not equivalent to the oscillation formula (12), unless the masses $m_{i, j}$ are nearly degenerate.

We now proceed to show that mass prefactors only appear at an intermediate stage of the calculation, but not in the final result. Note first that the amplitude (171) is in correspondence with our amplitude (95), computed with the stationary phase method, since the prefactor in Eq. (133) can be rewritten, for $T \gg T_{j}^{\text {disp }}$, as

$$
\frac{\sigma_{p e f f}}{T \sqrt{1+i T / T_{j}^{d i s p}}} \sim \frac{\sqrt{m_{j}}}{\left(T^{2}-L^{2}\right)^{3 / 4}},
$$

where the definition (92) of the dispersion time has been used. This prefactor coincides with the prefactor in Eq. (171). However, the subsequent time average of the transition probability completely cancels this dependence on the mass, yielding Eq. (144). This can be seen by expanding the argument of the exponential in Eq. (133) around the average propagation time. The width with respect to $T$ of the amplitude $\mathcal{A}_{j}$ is found to be equal to $\frac{\sigma_{p e f f} T}{m_{j} v_{0} \gamma_{c l}^{3}}$ (where $\gamma_{c l}$ is the Lorentz factor associated with the velocity $\left.v_{c l}=L / T\right)$, thereby providing a $m_{j}$-dependent factor that cancels the $m_{j}$ prefactor in the Gaussian integration.

Shtanov does not obtain such a result, since he does not perform any time average on the probability. Besides, mass prefactors would remain even if a time average were performed on the probability: no 'wave packet envelope' appears indeed in Shtanov's amplitude, so that the cancellation mechanism explained in the previous paragraph is not at work. For that reason, Shtanov wrongly concludes that the mass prefactor remains if the source is strongly localized. Another non-standard oscillation formula, derived by Shtanov for mass eigenstates satisfying $m_{2} \sqrt{\left(x^{\prime}-x\right)^{2}} \ll 1 \ll m_{1} \sqrt{\left(x^{\prime}-x\right)^{2}}$, reduces to the standard formula when decoherence mechanisms are taken into account. In conclusion, Shtanov's computations in configuration space do not lead to new oscillation formulas.

\subsubsection{No plane waves}

Ioannisian and Pilaftsis [163] derive a formula for neutrino oscillations which exhibits a plane wave behavior if the condition $L \lesssim p_{0} / \sigma_{p}^{2}$ is satisfied (with $\sigma_{x} \ll L_{i j}^{o s c}$, as usual). Their term 'plane wave oscillations' means that the oscillation amplitude $\mathcal{A}_{j}$ does not decrease as the inverse of the distance, that its phase depends on the direction $\mathbf{L}$ as $\mathbf{p}_{\mathbf{j}} \cdot \mathbf{L}$, and that there is no geometrical constraint linking the average 3-momentum and the direction of observation. If it were true, the oscillation length measured by a specific detector would depend on the direction of the total momentum of the initial particles. The plane wave condition is satisfied, for example, by taking $\sigma_{x} \sim 1 \mathrm{~cm}, L \sim 1000 \mathrm{~km}$ and $p \sim 1 \mathrm{GeV}$. These conditions might be verified for atmospheric neutrinos. 
In section 6.1.2, we have proved that the detection probability of an oscillating particle, derived under the condition $L \lesssim p_{0} / \sigma_{p}^{2}$, is negligible in directions other than the average momentum whether the stationary limit is taken or not. Therefore the oscillation phase has the standard form given in Eq. (14) and no 'plane wave oscillations' can be observed, contrary to the Ioannisian and Pilaftsis' claim. Also, the absence of the $1 / T$ (or $1 / L$ ) factor in Eq. (77), as noted by the same authors, is easily understood by noting that the absence of dispersion entails that the amplitude does not decrease with the distance (the probability is maximal within a cylinder as pictured on Fig. 6 of section 7.1.1).

It is interesting to understand why a directional constraint is missing for $L \lesssim p_{0} / \sigma_{p}^{2}$ in Ioannisian and Pilaftsis' result, as this fact explains their 'plane wave oscillations' prediction. At the end of their computations, Ioannisian and Pilaftsis obtain an amplitude $\mathcal{A}_{j}$ whose dominant term depends on $\exp \left(i p_{j}\left|\mathbf{L}^{\prime}\right|\right)$, where $\left|\mathbf{L}^{\prime}\right|=\sqrt{\mathbf{L}^{\prime 2}}$ is the complex 'norm' of a complex vector $\mathbf{L}^{\prime}=\mathbf{L}-2 i \sigma_{x}^{2} \mathbf{p}_{0}$ (their results are translated into our notation through the correspondence $\left.q_{j} \rightarrow p_{j}, \vec{k} \rightarrow \mathbf{p}_{0}, \delta l^{2} \rightarrow 4 \sigma_{x}^{2}, \vec{L} \rightarrow \mathbf{L}^{\prime}, \vec{l} \rightarrow \mathbf{L}\right)$. For $L \ll p_{0} / \sigma_{p}^{2}$, the quantity $\left|\mathbf{L}^{\prime}\right|$ can be expanded and the argument of the exponential reads to second order

$$
i p_{j}\left|\mathbf{L}-2 i \sigma_{x}^{2} \mathbf{p}_{0}\right| \cong 2 \sigma_{x}^{2} p_{0} p_{j}+i p_{j} \frac{\mathbf{p}_{0} \cdot \mathbf{L}}{p_{0}}-\frac{p_{j}}{4 \sigma_{x}^{2} p_{0}}\left(L^{2}-\frac{\left(\mathbf{p}_{0} \cdot \mathbf{L}\right)^{2}}{p_{0}^{2}}\right)
$$

The two last terms were neglected in Ref. [163], though $L \gg \sigma_{x}$. They lead to the following directional constraint:

$$
\exp \left(-\frac{\left(\mathbf{v}_{0} \times \mathbf{L}\right)^{2}}{4 \sigma_{x}^{2} v_{0}^{2}}\right),
$$

where the factor 4 becomes a factor 2 when the amplitude is squared. Therefore Ioannisian ad Pilaftsis' result includes a directional constraint forbidding plane wave oscillations.

Note that the stationary limit assumption $\left|\mathbf{v}_{P, D}\right| / \sigma_{e P, D}=0$ leads to $s_{1}=s_{2}$ (see Eq. (109)), so that the constraint (172) becomes equal to the one present in Eq. (111). Thus the condition $\left|\mathbf{v}_{P, D}\right| / \sigma_{e P, D}=0$ seems generic for models with stationary boundary conditions, since it was also applied in the case of the Grimus-Stockinger model (see discussion after Eq. (58) and at the end of section 4.5.2).

In conclusion, the analysis of the transition probability (111) derived in the external wave packet model under condition (75) has shown that 'plane wave oscillations' do not exist. This negative result was confirmed by a reexamination of the formula derived in Ref. 163.

\subsection{Conclusion}

Through the discussions of this section, the external wave packet model has shown its power and generality. This model can either reproduce in some limit the field-theoretical oscillation formulas found in the literature, or allows to understand why they are wrong. Thus the three categories of models described in section 3.1 (external wave packet models, stationary boundary condition models,source-propagator models) really make one. Furthermore, the external wave packet model has the advantage of associating a clear physical picture to the different stages 
of the calculations. For example, the role played by the dispersion in the observability conditions (coherence length and localization) could be cleared up in section 7.1, while the threshold

$L=p_{0} / \sigma_{p}^{2}$ identified by Ioannisian and Pilaftsis could be interpreted as indicating the onset of dispersion.

\section{Oscillations of unstable particles}

First, we study nonexponential corrections specific to mixed unstable particles, and then examine in which respect the oscillation formula obtained in the stable case is modified by the decay term. Next, we establish the relation between our formalism and the Wigner-Weisskopf-Lee-OehmeYang model. Finally, we apply the formulas derived in this section to the $B^{0} \bar{B}^{0}$ system.

\subsection{Nonexponential corrections due to mixing}

If the oscillating particle is unstable, the flavor-mixing amplitude (34) is a superposition of mass-eigenstate amplitudes $\mathcal{A}_{j}$ given by Eq. (35). However these formulas were derived on the assumption that the diagonalization matrices $V$ appearing in Eq. (34) are constant. This hypothesis is only true in the limit of a negligible renormalized self-energy. This approximation cannot be made for unstable particles, since their decay arises from the imaginary part of this self-energy. Actually, nonexponential corrections are always important at large time for unstable particles (though until now not observable), so it is worth checking whether the energymomentum dependence of the diagonalization matrix has a measurable effect on the transition probability.

Nonexponential corrections to the propagation of an unmixed particle of well-defined mass are studied in the Appendix, in the context of the derivation of the Jacob-Sachs theorem. These corrections have two origins. First, they are due to the bounded character of the energymomentum spectrum of the particle. Second, they are due to multi-particle thresholds, if these are included in the energy-momentum spectrum.

The first type of correction is easily calculated for mixed propagators, since it has nothing to do with the diagonalization matrices. The corrections can be evaluated separately for each partial amplitude $\mathcal{A}_{i}$, and are negligible (see Appendix). In order to compute the second type of corrections, the energy-dependent diagonalization matrices are kept inside the energymomentum integral (35). Let us consider the case of $P^{0} \bar{P}^{0}$ mixing in the limit of no CP violation ( $P$ is a $K$ or a $B$ meson). Starting from Eq. (31), the inverse propagator for the neutral meson system can be parametrized by [238]

$$
i G^{-1}\left(p^{2}\right)=\left(\begin{array}{cc}
p^{2}-m^{2}-f_{00}\left(p^{2}\right) & -f_{0 \overline{0}}\left(p^{2}\right) \\
-f_{\overline{0} 0}\left(p^{2}\right) & p^{2}-m^{2}-f_{00}\left(p^{2}\right)
\end{array}\right),
$$

where $m$ is the renormalized mass in the degenerate limit and $-i f_{\alpha \beta}\left(p^{2}\right)$ are the renormalized complex self-energies. The diagonal elements are equal because of CPT symmetry. In the limit of CP symmetry, the off-diagonal can be taken to be equal: $f_{0 \overline{0}}\left(p^{2}\right) \stackrel{C P}{=} f_{\overline{0} 0}\left(p^{2}\right)$. 
This propagator can be diagonalized as follows:

$$
-i G\left(p^{2}\right)=V^{-1}\left(p^{2}\right)\left(\begin{array}{cc}
\left(p^{2}-m_{1}^{2}-f_{1}\left(p^{2}\right)\right)^{-1} & 0 \\
0 & \left(p^{2}-m_{2}^{2}-f_{2}\left(p^{2}\right)\right)^{-1}
\end{array}\right) V\left(p^{2}\right) .
$$

Thresholds of multi-particle states appear in the renormalized self-energies $f_{j}\left(p^{2}\right)$ and in the diagonalization matrix $V\left(p^{2}\right)$.

Let us define the function $I_{\beta \alpha}(T)$ by

$$
I_{\beta \alpha}(T)=\int d E \psi(E, \mathbf{p}) G_{\beta \alpha}\left(p^{2}\right) e^{-i E T},
$$

where $G_{\beta \alpha}\left(p^{2}\right)$ is the nondiagonal propagator in flavor space and $\psi(E, \mathbf{p})$ is the overlap function defined by Eq. (28). The Fourier transform of this function with respect to the 3 -momentum yields the amplitude $\mathcal{A}(\alpha \rightarrow \beta, T, \mathbf{L})$. Since we are interested by nonexponential corrections to mixing, let us study the effect of a threshold $p^{2}=b^{2}$ on the matrix element $I_{0 \overline{0}}(T)$. The corresponding propagator matrix element is

$$
G_{0 \overline{0}}\left(p^{2}\right)=\frac{i V_{01}^{-1}\left(p^{2}\right) V_{1 \overline{0}}\left(p^{2}\right)}{p^{2}-m_{1}^{2}-f_{1}\left(p^{2}\right)}+\frac{i V_{02}^{-1}\left(p^{2}\right) V_{2 \overline{0}}\left(p^{2}\right)}{p^{2}-m_{2}^{2}-f_{2}\left(p^{2}\right)} .
$$

The function $I_{\beta \alpha}(T)$ is computed with the method explained in the Appendix. The integration on the energy is done by a contour integration including the two poles $z_{1,2}$ appearing in the right-hand side of Eq. (174). The function $I_{\beta \alpha}(T)$ is equal to the sum of the poles' contribution $J$, the contributions $J_{1,2}$ of the energy-momentum thresholds of the overlap function $\psi(E, \mathbf{p})$, as well as the contribution $J_{b}$ of the multi-particle threshold:

$$
I_{0 \overline{0}}(T)=J+J_{1}+J_{2}+J_{b}
$$

$J$ is given by the residues of the two poles $z_{j}=m_{j}^{2}-i m_{j} \Gamma_{j}$ :

$$
J=V_{01}^{-1}\left(z_{1}\right) V_{1 \overline{0}}\left(z_{1}\right) \frac{\pi \psi\left(z_{1}, \mathbf{p}\right)}{\sqrt{z_{1}+\mathbf{p}^{2}}} e^{-i \sqrt{z_{1}+\mathbf{p}^{2}} T}+V_{02}^{-1}\left(z_{1}\right) V_{2 \overline{0}}\left(z_{2}\right) \frac{\pi \psi\left(z_{2}, \mathbf{p}\right)}{\sqrt{z_{2}+\mathbf{p}^{2}}} e^{-i \sqrt{z_{2}+\mathbf{p}^{2}} T} .
$$

Since CP violation is neglected, the propagation eigenstates are the CP eigenstates $K_{1,2} \sim$ $K^{0} \pm \bar{K}^{0}$, so that the matrix $V$ is given at the pole by

$$
V\left(z_{1}\right) \cong V\left(z_{2}\right) \cong \frac{1}{\sqrt{2}}\left(\begin{array}{rr}
1 & 1 \\
1 & -1
\end{array}\right)
$$

We shall take into account, in the evaluation of $J_{b}$, that these expressions are not valid far from the poles. An estimate of $J$ is given by

$$
J \sim \frac{1}{m} \psi\left(m^{2}, \mathbf{p}\right) e^{-\Gamma_{2} T / 2}\left|e^{-i \Delta m T-\Delta \Gamma T / 2}-1\right|,
$$

where $m$ is the mass in the degenerate limit, $\Delta m=m_{1}-m_{2}, \Delta \Gamma=\Gamma_{1}-\Gamma_{2}$ and we have approximated $\sqrt{m^{2}+\mathbf{p}^{2}} \cong m$. 
The contributions $J_{1}$ and $J_{2}$, due to the bounded spectrum of the overlap function, can be computed separately on each term of the right-hand side of Eq. (174), exactly as in the one-particle case. The result is likewise negligible.

Let us examine in detail the contribution of $J_{b}$. Eq. (216) of the Appendix shows that $J_{b}$ depends on the difference between the complete propagator $G_{\beta \alpha}(z)$ and its analytic continuation $G_{\beta \alpha, I I}(z)$ :

$$
J_{b}=-i e^{-i \sqrt{b^{2}+\mathbf{p}^{2}} T} \int_{0}^{\infty} d \omega \psi(z(\omega), \mathbf{p})\left(G_{0 \overline{0}, I I}(z)-G_{0 \overline{0}}(z)\right) e^{-\omega T} .
$$

The difference $G_{I I}(z)-G(z)$ can be computed with the parametrization (173). To first order in $f_{\alpha \beta}$, we have

$$
G_{0 \overline{0}, I I}(z)-G_{0 \overline{0}}(z)=-i\left(f_{0 \overline{0}, I I}(z)-f_{0 \overline{0}}(z)\right) G_{0 \overline{0}}(z) G_{0 \overline{0}, I I}(z) .
$$

For large $T$, the dominant contribution to $J_{b}$ comes from the $\omega$ values near zero, that is $z=b^{2}$, because of the decreasing exponential. It is thus enough to know how the integrand tends to zero near the threshold $z=b^{2}$. Just under the real axis $(z=x-i \epsilon)$, the spectral representation of the self-energy given by Eq. (213) entails that

$$
f_{0 \overline{0}, I I}(z)-f_{0 \overline{0}}(z)=f_{0 \overline{0}}(x+i \epsilon)-f_{0 \overline{0}}(x-i \epsilon)=2 i \mathcal{I m} f_{0 \overline{0}}(x+i \epsilon) .
$$

The value at the pole of the function $f_{0 \overline{0}}(x+i \epsilon)$ can be related to experimentally known quantities with the help of Eqs. (173) and (175):

$$
\mathcal{I}_{m} f_{0 \overline{0}}\left(m^{2}+i \epsilon\right) \cong-\frac{m \Delta \Gamma}{2} .
$$

As the main decay channels of $K^{0}$ and $\bar{K}^{0}$ are two-pion decays, the nondiagonal self-energy Im $f_{0 \overline{0}}(x+i \epsilon)$ has a two-particle threshold behavior:

$$
\mathcal{I} m f_{0 \overline{0}}(x+i \epsilon) \cong-\frac{m \Delta \Gamma}{2} \sqrt{\frac{x-b^{2}}{m^{2}-b^{2}}} .
$$

Eq. (178) can then be rewritten as

$$
G_{0 \overline{0}, I I}(z)-G_{0 \overline{0}}(z) \cong-m \Delta \Gamma \sqrt{\frac{z-b^{2}}{m^{2}-b^{2}}} G_{0 \overline{0}}(z) G_{0 \overline{0}, I I}(z) .
$$

Substituting $y=\omega T$ in Eq. (177) and expanding the integrand in $1 / T$ with the help of the $z(\omega)$ parametrization given by Eq. (217), we obtain

$$
z=b^{2}-2 i \frac{y}{T} \sqrt{b^{2}+\mathbf{p}^{2}}+\mathcal{O}\left(T^{-2}\right) .
$$

It follows that

$$
J_{b} \sim T^{-3 / 2} \frac{m^{3 / 2} \Delta \Gamma}{\left(m^{2}-b^{2}\right)^{5 / 2}} \psi\left(b^{2}, \mathbf{p}\right) e^{-i \sqrt{b^{2}+\mathbf{p}^{2}} T},
$$

where we have approximated $b^{2}+\mathbf{p}^{2} \cong m^{2}$ and used the fact that the self-energy is negligible at the threshold. Thus $J_{b}$ is of the order of

$$
J_{b} \sim(Q T)^{-3 / 2} \frac{\Delta \Gamma}{Q} \frac{1}{m} \psi\left(b^{2}, \mathbf{p}\right) .
$$

It is now possible to compare the estimates of $J$ and $J_{b}$, given by Eqs. (176) and (179), respectively. 
At small times: $J_{b} \sim J$ if

$$
(Q T)^{-3 / 2} \frac{|\Delta \Gamma|}{Q} \sim\left|\sin \frac{\Delta m T}{2}\right| \sim \frac{|\Delta m| T}{2} .
$$

It is known that $|\Delta \Gamma| \lesssim|\Delta m|$ (from the experimental data in the case of the $K$ mesons 156] and from theoretical predictions in the case of $B$ mesons [115). Thus, $J_{b} \sim J$ if $T \sim Q^{-1}$. Since $Q$ is of the order of $0.2-1 \mathrm{GeV}$, for the $K$ and $B$ mesons, nonexponential corrections are dominant at small times for $T \lesssim 10^{-24}$ s. This time range is not observable.

At large times: $J \sim J_{b}$ if

$$
e^{-\Gamma_{L} T / 2} \sim(Q T)^{-3 / 2} \frac{|\Delta \Gamma|}{Q} \sim\left(\Gamma_{L} T\right)^{-3 / 2} \frac{|\Delta \Gamma| \Gamma_{L}^{3 / 2}}{Q^{5 / 2}},
$$

where $\Gamma_{L}=\min \left(\Gamma_{1}, \Gamma_{2}\right)$, that is if

$$
\Gamma_{L} T-3 \ln \left(\Gamma_{L} T\right) \sim 5 \ln \left(\frac{Q}{\Gamma_{L}}\right)-2 \ln \left(\frac{|\Delta \Gamma|}{\Gamma_{L}}\right) .
$$

For kaons, $Q \sim 220 \mathrm{MeV}$ so that $\Gamma_{L} T \sim 190$. The amplitude is negligible at such large times so that nonexponential corrections are not observable. For $B$ mesons, $Q \sim 1 \mathrm{GeV}$ and $\Delta \Gamma / \Gamma_{L}$ can be estimated at $4 \times 10^{-3}$ for the $B_{d}^{0}$ and at $10^{-1}$ for the $B_{s}^{0}$ [115], so that $\Gamma_{L} T \sim 168$ in the former case and $\Gamma_{L} T \sim 162$ in the latter. Thus, nonexponential corrections are not observable in either case.

The estimate (179) of nonexponential corrections to the propagation of mixed neutral mesons is in agreement with the theoretical formulas obtained by Chiu and Sudarshan (Eq. (3.30) of [88]), and by Wang and Sanda (Eq. (59) of [254]). The authors of these two papers compute nonexponential corrections in a quantum-mechanical framework and extend the WignerWeisskopf-Lee-Oehme-Yang formalism 191, 172, 209] (see also 215]).

In conclusion, nonexponential corrections can be neglected in the propagation of quasistable mixed particles. Therefore, the flavor-mixing amplitude (34) with constant matrices $V$ can be used not only in the stable case, but also for an unstable oscillating particle.

\subsection{Oscillation formula for unstable particles}

The approximation of the amplitude $\mathcal{A}_{j}$ and of the time average with Laplace's or the stationary phase methods can be transposed for an unstable oscillating particle. The decay term $\gamma_{j}(p)$ introduces a new small parameter, namely $m_{j} \Gamma_{j} / p^{2}$. This parameter is of order $\epsilon=\delta m_{i j}^{2} / 2 p^{2}$, or smaller, since the mean decay length $L_{j}^{\text {decay }}=p / m_{j} \Gamma_{j}$ should be of the order, or larger, than the oscillation length $L_{i j}^{o s c}=4 \pi p / \delta m_{i j}^{2}$, or else the oscillations would not be observable. In parallel with the discussion of section 5.3.1, the influence of this new parameter on the transition probability shows up in the argument of the exponential, through combinations with large dimensionless coefficients. 
Let us inspect the possible combinations of $\epsilon$ with the available large parameters, where $\epsilon$ refers either to $\left(p L^{o s c}\right)^{-1}$ or to $\left(p L^{\text {decay }}\right)^{-1}$. First, if the conditions of production and detection are not considered, only one such coefficient is available, i.e. $p L$. This combination gives the oscillation term $\exp \left(-2 \pi i L / L^{o s c}\right)$ and the usual exponential decrease of the probability in $\exp \left(-L / L^{\text {decay }}\right)$. Next, the analysis of the propagation with external wave packets adds a new parameter, i.e. $\sigma_{p e f f}$. The combinations $\left(\epsilon p / \sigma_{p e f f}\right)^{2}$ and $\left(\epsilon \sigma_{p e f f} L\right)^{2}$ give the following contributions to the argument of the exponential (with minus signs omitted):

1. Decay-independent terms, like the decoherence term, $\left(\frac{L}{L^{c o h}}\right)^{2}$, and the localization term, $\left(\frac{\sigma_{x e f f}}{L^{\text {osc }}}\right)^{2}$, which were already obtained in the stable case.

2. Mixing-independent terms, like $\left(\frac{\sigma_{\text {peff }}}{p} \frac{L}{L^{\text {decay }}}\right)^{2}$ and $\left(\frac{\sigma_{x e f f}}{L^{\text {decay }}}\right)^{2}$. The first is completely negligible in comparison with the exponential decrease at order $\epsilon$. The second imposes that $L^{\text {decay }} \gg \sigma_{x e f f}$, which is true for quasi-stable particles.

3. Mixing-decay cross terms, like $\frac{\sigma_{x e f f}^{2}}{L^{\text {osc }} L^{\text {decay }}}$ and $\frac{\sigma_{p e f f}^{2}}{p^{2}} \frac{L^{2}}{L^{\text {osc }} L^{\text {decay }}}$.

With the help of the condition $\mathcal{O}\left(L^{\text {decay }}\right) \gtrsim \mathcal{O}\left(L^{o s c}\right)$, it can be seen that the new contributions, with respect to the stable case, do not impose new constraints for the observability of the oscillations. They are thus neglected in the transition probability.

We have yet to check whether the oscillation phase is modified or not by the widths $\Gamma_{j}$. Let us consider first the transversal-dispersion regime (the no-dispersion regime can be treated similarly). The position $p_{j}$ of the maximum is shifted by the decay term $\gamma_{j}(p)$. The new maximum is the solution of

$$
f_{j}^{\prime}(p)+\gamma_{j}^{\prime}(p)=0
$$

where $f_{j}(p)$ and $\gamma_{j}(p)$ are defined by Eqs. (82) and (84), respectively. It can be computed as before to first order in $\epsilon$ ( $\epsilon$ referring to $\delta m_{j}^{2} / 2 E_{0}^{2}$ or to $\left.m_{j} \Gamma_{j} / 2 E_{0}^{2}\right)$ and is equal to

$$
p_{j}=p_{j}^{(0)}+\delta p_{j}^{(\Gamma)},
$$

where $p_{j}^{(0)}$ is the solution for $\Gamma_{j}=0$, given by Eq. (114), whereas $\delta p_{j}^{(\Gamma)}$ is the contribution from the decay term, given by

$$
\delta p_{j}^{(\Gamma)} \lesssim p_{0} \frac{\sigma_{p e f f}^{2}}{E_{0}^{2}} \frac{T}{T_{j}^{\text {decay }}} .
$$

The decay time is defined by $T_{j}^{\text {decay }}=E_{0} / m_{j} \Gamma_{j}$. Although the expansion parameter $m_{j} \Gamma_{j} / 2 E_{0}^{2}$ is multiplied in the exponential by the large parameter $T$, the shift $\delta p_{j}^{(\Gamma)}$ is small and the expansion (180) is valid as long as the propagation time $T$ is not much larger than the decay time $T_{j}^{\text {decay }}$. The corresponding energy $E_{j}=\sqrt{p_{j}^{2}+m_{j}^{2}}$ and velocity $v_{j}=p_{j} / E_{j}$ are given by

$$
\begin{aligned}
E_{j} & =E_{j}^{(0)}+\delta E_{j}^{(\Gamma)}, \\
v_{j} & =v_{j}^{(0)}+\delta v_{j}^{(\Gamma)},
\end{aligned}
$$


where $E_{j}^{(0)}$ and $v_{j}^{(0)}$ are the solutions for $\Gamma_{j}=0$, given by Eqs. 116) and (117), respectively, whereas the contributions from the decay term are equal to $\delta E_{j}^{(\Gamma)}=v_{0} \delta p_{j}^{(\Gamma)}$ and $\delta v_{j}^{(\Gamma)}=m_{0}^{2} \delta p_{j}^{(\Gamma)} / E_{0}^{3}$.

Apart from the modified values of $p_{j}, E_{j}$ and $v_{j}$, the longitudinal momentum integration with Laplace's method yields the same results as in section 6.2.1. The phase of the interference term $\mathcal{A}_{i} \mathcal{A}_{j}^{*}$ is thus given, before the time average, by Eq. $(120)$, but with the modified values (180), (182) and (183). It can be split in a $\Gamma$-independent part $\phi_{i j}^{(0)}(T, L)$, and a $\Gamma$-dependent part $\phi_{i j}^{(\Gamma)}(T, L)$. The latter is equal to

$$
\phi_{i j}^{(\Gamma)}(T, L)=\left(v_{0} T-L\right)\left(\delta p_{i}^{(\Gamma)}-\delta p_{j}^{(\Gamma)}\right)\left(1-\frac{\ell^{2}}{1+\ell^{2}}\right),
$$

where $\ell=T / T^{\text {disp }}$ and the definition (92) of $T^{\text {disp }}$ has been used. Using the condition $\left|v_{0} T-L\right| \lesssim$ $\sigma_{\text {xeff }}$ (see Eq. (113)) and the values of $p_{i, j}^{(\Gamma)}$ given by Eqs. (180) and (181), we have

$$
\left|\phi_{i j}^{(\Gamma)}(T, L)\right| \lesssim v_{0} \frac{\sigma_{\text {peff }}}{E_{0}}\left|\frac{T}{T_{i}^{\text {decay }}}-\frac{T}{T_{j}^{\text {decay }}}\right|,
$$

which is much smaller than 1 in the observable domain $\mathcal{O}(T) \lesssim \mathcal{O}\left(T^{\text {decay }}\right)$. Therefore, the oscillation phase is not modified by the width:

$$
\left|\phi_{i j}^{(\Gamma)}(T, L)\right| \ll\left|\phi_{i j}^{(0)}(T, L)\right| .
$$

Let us now turn our attention to the longitudinal-dispersion regime. Of course, the stationary point $p_{c l, j}$ is not shifted by the decay term $\gamma_{j}(p)$, but the phase receives a contribution from the derivative of the decay term. More precisely, the phase (138) becomes

$$
\tilde{\phi}_{i j}(T, L)=\delta m_{i j} \sqrt{T^{2}-L^{2}}+\frac{\sigma_{p e f f}^{2} \ell}{1+\ell^{2}}\left(\left(f_{i}^{\prime}\left(p_{c l, i}\right)+\gamma_{i}^{\prime}\left(p_{c l, i}\right)\right)^{2}-\left(f_{j}^{\prime}\left(p_{c l, j}\right)+\gamma_{j}^{\prime}\left(p_{c l, j}\right)\right)^{2}\right)
$$

where $\ell=T / T^{\text {disp }}$ as above. The derivative of the decay term is equal to

$$
\gamma_{j}^{\prime}\left(p_{c l, j}\right)=-\frac{p_{0}}{2 E_{c l, j}^{2}} \frac{L}{L_{j}^{\text {decay }}} .
$$

The terms depending on $\Gamma_{i, j}$ are proportional to $\sigma_{p e f f} \gamma_{j}^{\prime}$. They will be small in the observable domain $\mathcal{O}(T) \lesssim \mathcal{O}\left(T^{\text {decay }}\right)$, so that they can be neglected in the phase (185).

Thus the oscillation phase is not modified by the decay term at any distance. The result (152), obtained for a stable oscillating particle, is only modified by the usual exponential decrease $\exp \left(-L / L_{i j}^{\text {decay }}\right)$, with

$$
L_{i j}^{\text {decay }}=\frac{2 p_{0}}{m_{i} \Gamma_{i}+m_{j} \Gamma_{j}} .
$$

In conclusion, the flavor-mixing transition probability for a long-lived unstable particle (i.e. satisfying $\sigma_{x e f f} \ll L_{i j}^{\text {decay }}$ ), having a relativistic or a nonrelativistic velocity (but always with 
$\left.\delta m_{i j}^{2} \ll E_{0}^{2}\right)$, and with dispersion taken into account, is given for $L \gtrsim p_{0} / \sigma_{p}^{2}$ by

$$
\begin{aligned}
& \mathcal{P}_{\alpha \rightarrow \beta}(\mathbf{L})=\frac{N_{g} g^{2}(\mathbf{l})}{L^{2}} \sum_{i, j} V_{i \alpha} V_{\beta i}^{-1} V_{j \alpha}^{*} V_{\beta j}^{-1 *} \exp \left(-\frac{L}{L_{i j}^{\text {decay }}}\right) \\
& \quad \times \exp \left(-2 \pi i \frac{L}{L_{i j}^{\text {osc }}}-\frac{\left(\delta m_{i}^{2}\right)^{2}+\left(\delta m_{j}^{2}\right)^{2}}{16 \sigma_{m}^{2} E_{0}^{2}}-2 \pi^{2}\left(\frac{\rho \sigma_{x e f f}}{L_{i j}^{\text {osc }}}\right)^{2}-\left(\frac{L}{L_{i j}^{\text {coh }}}\right)^{2}\right) .
\end{aligned}
$$

The definitions of the characteristic lengths and parameters appearing in this formula are gathered below Eq. (152). For $L \lesssim p_{0} / \sigma_{p}^{2}$, the oscillation formula for stable oscillating particles is similarly modified by the insertion of a decay term into Eq. (111).

\subsection{Wigner-Weisskopf effective mass matrix}

Oscillating neutral mesons are usually described in quantum mechanics with the Wigner-Weisskopf method as extended by Lee, Oehme and Yang [191, 172, 209]. In this framework, the evolution of a two-meson state satisfies a Schrödinger-like equation with a complex (and nonHermitian) effective mass matrix $H$, which can be split into a Hermitian mass matrix $M$ and a Hermitian decay matrix $\Gamma, H=M-i \Gamma$. It would be interesting to establish a correspondence between this method and the field-theoretical formula (187).

First, let us simplify the oscillation formula (187). Note that the coherence length can be neglected for particles such as the neutral $K$ or $B$ mesons, since the oscillation length is comparable with the decay length:

$$
\frac{L_{i j}^{c o h}}{L_{i j}^{\text {decay }}} \sim \frac{p_{0}}{\sigma_{p e f f}} \frac{L_{i j}^{o s c}}{L_{i j}^{\text {decay }}} \gg 1 .
$$

Next, the quasi-degeneracy of the mass eigenstates makes it impossible to set up experimental conditions which destroy oscillations. Thus the localization terms (containing the parameters $\sigma_{m}$ and $\rho$ ) drop from Eq. (187). These approximations lead to the following simplified formula:

$$
\mathcal{P}_{\alpha \rightarrow \beta}(\mathbf{L})=\frac{N_{g} g^{2}(\mathbf{l})}{L^{2}} \sum_{i, j} V_{i \alpha} V_{\beta i}^{-1} V_{j \alpha}^{*} V_{\beta j}^{-1 *} \exp \left(-\frac{L}{L_{i j}^{\text {decay }}}-2 \pi i \frac{L}{L_{i j}^{o s c}}\right) .
$$

The comparison of Eq. (188) with the Wigner-Weisskopf-Lee-Oehme-Yang theory is more easily done at the level of the amplitude. Whereas the true amplitude depends on both $T$ and $\mathbf{L}$, the probability (188) can, equivalently, be generated from the following effective amplitude

$$
\mathcal{A}_{e f f}(\alpha \rightarrow \beta, \mathbf{L})=\frac{\sqrt{N_{g}} g(\mathbf{l})}{L} \sum_{j} V_{\beta j}^{-1} \exp \left(-i\left(m_{j}-i \frac{\Gamma_{j}}{2}\right) \frac{m_{0} L}{p_{0}}\right) V_{j \alpha},
$$

where the masses are assumed to be nearly degenerate $\left(m_{i} \cong m_{j} \cong m_{0}\right)$. If $M$ is a diagonal matrix the diagonal terms of which are given by $m_{j}-i \Gamma_{j} / 2$, the effective amplitude can be 
written as

$$
\begin{aligned}
\mathcal{A}_{\text {eff }}(\alpha \rightarrow \beta, \mathbf{L}) & =\frac{\sqrt{N_{g}} g(\mathbf{l})}{L}\left(V^{-1} \exp \left(-i M \frac{m_{0} L}{p_{0}}\right) V\right)_{\beta \alpha} \\
& =\frac{\sqrt{N_{g}} g(\mathbf{l})}{L}\left(\exp \left(-i M_{\text {flavor }} \frac{m_{0} L}{p_{0}}\right)\right)_{\beta \alpha},
\end{aligned}
$$

where $M_{\text {flavor }}=V^{-1} M V$. The mass matrix $M_{\text {flavor }}$ corresponds to the effective Hamiltonian in the Lee-Oehme-Yang theory. We recognize the factor $m_{0} L / p_{0}$ as being the classical proper time of propagation, common to the two mass eigenstates. However, let us again emphasize that this observation does not justify the equal time prescription, which was explained in section 2.3. It must not be forgotten that $T$ and $L$ are the average propagation time and length, and that interference takes place in a time and space range defined by the width of the effective "wave packet'.

The effective oscillation amplitude confirms the soundness of the reciprocal basis treatment in quantum mechanics explained in section 2.2. Recalling that the diagonalization matrix $V$ is related to the matrix $U$ diagonalizing the states through $V=U^{t}$, the Hamiltonian (10) can be written as

$$
\hat{H}_{\text {propag }}=\left|\nu_{\beta}>\sum_{j} V_{\beta j}^{-1} e^{-i \lambda_{j} t} V_{j \alpha}<\nu_{\alpha}\right|,
$$

where $\lambda_{j}=m_{j}-i \Gamma_{j} / 2$. If the time $t$ is taken to be $m_{0} L / p_{0}$, the matrix element $\left\langle\nu_{\beta}\left|\hat{H}_{\text {propag }}\right| \nu_{\alpha}\right\rangle$ is in correspondence with the amplitude $\mathcal{A}_{\text {eff }}(\alpha \rightarrow \beta, \mathbf{L})$ given by Eq. (189). The field-theoretical method will thus give the same results as the reciprocal basis formalism in the case of nearly degenerate unstable states. Both formalisms are easier to use that the cumbersome nonorthogonal basis (3), since any transition amplitude can be simply computed as a matrix product. Since the reciprocal basis formalism has already been applied to the study of CP violation (see 238, 110, 17, 77, 243]), we shall only give one example in section 8.4 of the application of formula (189) to the computation of a CP asymmetry.

Let us end this section by an remark on the observability of intermediate unstable particles. It is tempting to associate a well-defined mass eigenstate with the exponential behavior in $e^{-i \lambda_{j} t}$ in Eqs. (189) or (190), but normalized mass eigenstates cannot be defined if the mass matrix $M_{\text {flavor }}$ is not normal (see section 2.1). The reason is that a final state can be produced by all mass channels. In practice, it is sometimes possible to maximize the probability of a channel (since the lifetimes $\Gamma_{j}$ can be very different). In that case, one talks about decays into a mass eigenstate represented by its reciprocal basis vector out $<\nu_{j}(0) \mid$ [77].

\subsection{Oscillations in the $B^{0} \bar{B}^{0}$ system}

$B^{0} \bar{B}^{0}$ oscillations were first measured in 1987 [13], but the discovery of $\mathrm{CP}$ violation in this system is very recent [3, 30]. We compute here as an example the following CP asymmetry [213]:

$$
A_{C P}\left(B_{d}^{0} \rightarrow f, L\right)=\frac{\Gamma\left(B_{d}^{0}(L) \rightarrow f\right)-\Gamma\left(\bar{B}_{d}^{0}(L) \rightarrow f\right)}{\Gamma\left(B_{d}^{0}(L) \rightarrow f\right)+\Gamma\left(\bar{B}_{d}^{0}(L) \rightarrow f\right)},
$$


where the index $f$ refers to a CP eigenstate. The propagation eigenstates have nearly equal decay widths [115], and must be distinguished by their different masses. Let us define $\Delta m_{d} \equiv m_{H}-m_{L}$, where the indices refer to $B_{L}$, for Light, and $B_{H}$, for Heavy.

In the limit of a constant self-energy matrix $f_{\alpha \beta}$, the diagonalizing matrix of the mixed propagator $(173)$ can be parametrized by

$$
V=\frac{v}{\sqrt{2 \sigma}}\left(\begin{array}{rr}
\sigma & 1 \\
\sigma & -1
\end{array}\right) \quad \text { and } \quad V^{-1}=\frac{v^{-1}}{\sqrt{2 \sigma}}\left(\begin{array}{rr}
1 & 1 \\
\sigma & -\sigma
\end{array}\right),
$$

where $\sigma^{2}=f_{\overline{0} 0} / f_{0 \overline{0}}$. Note that the phase of this parameter depends on the phase convention chosen for flavor states. The physics should be invariant [77 under

$$
\left|B^{0}\right\rangle \rightarrow e^{i \gamma}\left|B^{0}\right\rangle \quad \text { and } \quad\left|\bar{B}^{0}\right\rangle \rightarrow e^{i \bar{\gamma}}\left|\bar{B}^{0}\right\rangle \text {. }
$$

Under the ket rephasing (193), $\sigma$ transforms as $\sigma \rightarrow e^{i(\gamma-\bar{\gamma})} \sigma$. This parameter is related to the notations of [77] through $\sigma=-q_{B} / p_{B}$. The deviation of $|\sigma|$ from 1 parametrizes the amount of $\mathrm{CP}$ violation in mixing, called 'indirect CP violation'. The constant $v$ in Eq. (192) is an arbitrary normalization, underlining the fact that the meson cannot be considered as an asymptotic state. Its decay amplitude should always be included in the full amplitude.

Theoretical calculations ([77] and references therein) show that $|\sigma|-1 \sim \mathcal{O}\left(10^{-4}\right.$ ), which is much smaller than the present experimental uncertainty, so that only a small $\mathrm{CP}$ violation occurs in the mixing. On the other hand, a much larger $\mathrm{CP}$ violation is expected in the decays ('direct CP violation'), since three quark generations are involved in processes such as $B_{d} \rightarrow J / \psi K_{S}$. Thus, CP violation in mixing is usually neglected for $B$ mesons (i.e. $|\sigma|=1$ ), which is the opposite of what occurs with neutral kaons.

The computation of the asymmetry (191) requires the knowledge of the amplitude $\mathcal{T}_{f}(L)$, corresponding to the propagation of an initial $B^{0}$. Starting from the effective oscillation amplitude $(189)$, we can write $\mathcal{T}_{f}(L)$ as

$$
\mathcal{T}_{f}(L) \sim\left(\mathcal{M}\left(B^{0} \rightarrow f\right) \quad \mathcal{M}\left(\bar{B}^{0} \rightarrow f\right)\right) V^{-1} \exp \left\{-i M \frac{m_{0} L}{p_{0}}\right\} V\left(\begin{array}{l}
\mathcal{C} \\
0
\end{array}\right),
$$

where the effective mass matrix $M$ is equal to $\operatorname{diag}\left(m_{L}-i \Gamma_{L} / 2, m_{H}-i \Gamma_{H} / 2\right)$. It will be useful to define the parameter $\mu_{f}$ by

$$
\mu_{f}=\frac{\mathcal{M}\left(\bar{B}^{0} \rightarrow f\right)}{\mathcal{M}\left(B^{0} \rightarrow f\right)}
$$

Under the rephasing (193) of the kets, $\mu_{f} \rightarrow e^{-i(\gamma-\bar{\gamma})} \mu_{f}$. If $\left|\mu_{f}\right| \neq 1$, 'direct CP violation' occurs in decay amplitudes to the state $f$. Whereas the quantities $\sigma$ and $\mu_{f}$ are not invariant under the rephasing (193), the product $\sigma \mu_{f}$ is invariant. This quantity is related to the notations of [77] through $\sigma \mu_{f}=-\lambda_{f}$. If the final state is a $\mathrm{CP}$ eigenstate and the transition dominated by a single CKM amplitude (for example $B_{d}^{0} \rightarrow J / \psi K_{S}$ ), $\sigma \mu_{f}$ can be expressed in terms of the elements of the quark mixing matrix, so that its measurement is very important for the determination the CKM matrix elements. 
The amplitude $\mathcal{T}_{f}(L)$ can be written as

$$
\mathcal{T}_{f}(L) \sim \frac{\mathcal{C M}\left(B_{0} \rightarrow f\right)}{2}\left(\left(1+\sigma \mu_{f}\right) e^{-i m_{L} \frac{m_{0}}{p_{0}} L-\frac{m_{0}}{2 p_{0}} \Gamma_{L} L}+\left(1-\sigma \mu_{f}\right) e^{-i m_{H} \frac{m_{0}}{p_{0}} L-\frac{m_{0}}{2 p_{0}} \Gamma_{H} L}\right)
$$

where $p_{0}$ is the modulus of the total momentum of the final pions.

We also need to compute the amplitude $\overline{\mathcal{T}_{f}}(L)$, corresponding to the propagation of an initial $\bar{B}^{0}$. The same method yields

$$
\overline{\mathcal{T}_{f}}(L) \sim \frac{\mathcal{C}^{*} \mathcal{M}\left(B_{0} \rightarrow f\right)}{2 \sigma}\left(\left(1+\sigma \mu_{f}\right) e^{-i m_{L} \frac{m_{0}}{p_{0}} L-\frac{m_{0}}{2 p_{0}} \Gamma_{L} L}-\left(1-\sigma \mu_{f}\right) e^{-i m_{H} \frac{m_{0}}{p_{0}} L-\frac{m_{0}}{2 p_{0}} \Gamma_{H} L}\right) .
$$

With the insertion of Eqs. (195)-(196) and the approximations $|\sigma|=1$ and $\Gamma_{H}=\Gamma_{L}$, the asymmetry (191) becomes

$$
A_{C P}\left(B_{d}^{0} \rightarrow f, L\right)=A_{C P}^{d i r}\left(B_{d}^{0} \rightarrow f\right) \cos \left(\Delta m_{d} \frac{m_{0} L}{p_{0}}\right)+A_{C P}^{\text {interf }}\left(B_{d}^{0} \rightarrow f\right) \sin \left(\Delta m_{d} \frac{m_{0} L}{p_{0}}\right),
$$

where direct $\mathrm{CP}$ violation, defined by

$$
A_{C P}^{d i r}\left(B_{d}^{0} \rightarrow f\right)=\frac{1-\left|\lambda_{f}\right|^{2}}{1+\left|\lambda_{f}\right|^{2}}=\frac{1-\left|\mu_{f}\right|^{2}}{1+\left|\mu_{f}\right|^{2}}
$$

has been separated from $\mathrm{CP}$ violation coming from the interference between the mixing and the decay:

$$
A_{C P}^{\text {interf }}\left(B_{d}^{0} \rightarrow f\right)=\frac{2 \operatorname{Im} \lambda_{f}}{1+\left|\lambda_{f}\right|^{2}}=-\frac{2 \operatorname{Im}\left(\sigma \mu_{f}\right)}{1+\left|\mu_{f}\right|^{2}} .
$$

Three different kinds of $\mathrm{CP}$ violation have been met in this section: indirect, direct and interference CP violations, signaled by $|\sigma| \neq 1,\left|\mu_{f}\right| \neq 1$ and $\operatorname{Im}\left(\sigma \mu_{f}\right) \neq 0$, respectively (see [77] p. 78). If the final state is $J / \psi K_{S}$, a theoretical study (see for example [115]) shows that the $\mathrm{CP}$ violation parameter is in a very good approximation equal to $\lambda_{J / \psi K_{S}}=e^{-2 i \beta}$, where $\beta$ is one of the angles of the unitary triangle. The $\mathrm{CP}$ violation in the neutral kaon system that is included in the final state can be neglected. The direct and interference asymmetries become

$$
A_{C P}^{\text {direct }}\left(B_{d}^{0} \rightarrow J / \psi K_{S}\right)=0 \quad \text { and } \quad A_{C P}^{\text {interf }}\left(B_{d}^{0} \rightarrow J / \psi K_{S}\right)=-\sin 2 \beta .
$$

The quantities $\sin 2 \beta$ and $|\lambda|$ were recently measured by the BABAR [30, 31] and Belle [3, 国] collaborations:

$$
\begin{aligned}
& \sin 2 \beta_{B A B A R}=0.741 \pm 0.067 \text { (stat) } \pm 0.033 \text { (syst) } \\
& \sin 2 \beta_{\text {Belle }}=0.719 \pm 0.074(\text { stat }) \pm 0.035 \text { (syst) } \\
& \left|\lambda_{B A B A R}\right|=0.948 \pm 0.051(\text { stat }) \pm 0.017 \text { (syst) } .
\end{aligned}
$$

The value of the interference asymmetry is an indication of $\mathrm{CP}$ violation in the $B$ system, whereas the value of $|\lambda|$ is consistent with no direct $\mathrm{CP}$ violation. 


\section{$9 \quad$ Selected topics}

In section 9.1, we examine the question of the normalization of the transition probability. We then discuss in section 9.2 the relation between coherent and incoherent effects, as well as the different estimates of the wave packet size. Finally, the case of an unstable source is reviewed in section 9.3 .

\subsection{Normalization of the transition probability}

Until now, we have not explained why the time-averaged squared modulus of the amplitude (see Eq. (96)) can be interpreted as the flavor-mixing transition probability. Actually, the interpretation of the expression (152) as a transition probability was a bit of a guess. As a matter of fact, the steps between the amplitude $\mathcal{A}(\alpha \rightarrow \beta)=\sum_{j} V_{\beta j}^{-1} \mathcal{A}_{j} V_{j \alpha}$ and a normalized event rate, containing an expression which can be interpreted as a flavor-mixing transition probability, are not straightforward. In the case of the large-distance oscillation formula, the constant prefactors $v_{0} N^{2} \sigma_{\text {peff }}$ appearing in Eq. (132) (or in Eqs. (144) and (151)) have been shoved into a constant $N_{g}$, which was normalized in Eq. (153) in order to obtain a unitary evolution in the stable case. The normalization of the short-distance oscillation formula (111) proceeded in the same way. Whereas these prefactors can be factorized from the sum on the mass eigenstates if $\delta m_{i j}^{2} \ll E_{0}^{2}$, this is not possible for a mixing of a relativistic $m_{i}$ and a nonrelativistic $m_{j}$ mass eigenstate.

Cardall has made an attempt 85 to go all the way to a normalized event rate. Although he claims to resort to arbitrary external wave packets, his model is equivalent to the Gaussian external wave packet model, since he uses Gaussian approximations to perform the integrals. We shall explain how Cardall's argument can be applied to the external wave packet model developed in sections 4 to 6 . Only the large-distance case will be considered $\left(L \gtrsim p_{0} / \sigma_{p}^{2}\right)$; the short-distance case can be treated similarly.

The expression $\mathcal{A}(\alpha \rightarrow \beta, T, \mathbf{L}) \mathcal{A}^{*}(\alpha \rightarrow \beta, T, \mathbf{L})$ (see Eq. (34)) is proportional to a transition probability between one-particle states. In order to obtain a formula applicable to experiments, the external wave packets should be interpreted as densities of particles. Cardall proposes three rules of correspondence:

1. Let us first examine the external wave packets normalization constants $N_{P_{\text {in }}}, N_{P_{\text {out }}}, N_{D_{\text {in }}}$ and $N_{D_{\text {out }}}$. They come from the overlap function and are included in the factor $N^{2}$ appearing in the transition probability (132). Eq. (52) shows that $N_{P_{i n}}$ is proportional to the modulus of the initial wave function at production. The constant $N_{D_{i n}}$ is interpreted in the same way. The constants $N_{P_{i n}}^{2}$ and $N_{D_{i n}}^{2}$ are then interpreted as the initial state particle densities at production and detection, respectively:

$$
N_{P_{i n}}^{2} \rightarrow \frac{d \mathbf{Q}}{(2 \pi)^{3} 2 E_{P_{i n}}} f\left(\mathbf{Q}, x_{P}\right) \quad \text { and } \quad N_{D_{i n}}^{2} \rightarrow \frac{d \mathbf{Q}^{\prime}}{(2 \pi)^{3} 2 E_{D_{i n}}} f\left(\mathbf{Q}^{\prime}, x_{D}\right),
$$

where $f$ is the phase space density. The normalization constants $N_{P_{\text {out }}}^{2}$ and $N_{D_{\text {out }}}^{2}$ are 
interpreted as particle densities for the final state particles at production and detection:

$$
N_{P_{\text {out }}}^{2} \rightarrow \frac{d \mathbf{K}}{(2 \pi)^{3} 2 E_{P_{\text {out }}}} \quad \text { and } \quad N_{D_{\text {out }}}^{2} \rightarrow \frac{d \mathbf{K}^{\prime}}{(2 \pi)^{3} 2 E_{D_{\text {out }}}} .
$$

Note that there may be more than one particle in the initial and final states.

2. Let us now consider the production and detection widths. Recall that the factor $N^{2}$ in Eq. (132) contains a volume factor $V^{2}=\left(2^{-4} \pi^{4} \sigma_{p P}^{-3} \sigma_{e P}^{-1} \sigma_{p D}^{-3} \sigma_{e D}^{-1}\right)^{2}$ coming from the overlap function (see Eq. (54)). One factor $V$ is interpreted as an integration on macroscopic spacetime variables:

$$
V \rightarrow d \mathbf{x}_{P} d x_{P}^{0} d \mathbf{x}_{D} d x_{D}^{0}
$$

Since the production time is not measured, it is integrated over in the event rate. This integration is equivalent to the time average over the propagation time $T$ done in the present report.

3. Finally, the effective width $\sigma_{\text {peff }}$, appearing in front of the exponential in Eq. (132), is interpreted as the result of an integration over the energy spectrum of the oscillating particle. Furthermore, the second volume factor $V$, the geometrical factor $g^{2}(\mathbf{l})$ and the term including $\sigma_{m}$ are interpreted, in the limit $m_{i}=m_{j} \equiv \tilde{m}_{0}$, as a product of delta functions constraining the direction of $\mathbf{L}$ and the energy of the oscillating particle. More precisely,

$$
v_{0} \sigma_{p e f f} V \exp \left(-\frac{\mathbf{p}_{0}^{2}-\left(\mathbf{p}_{0} \cdot \mathbf{l}\right)^{2}}{2 \sigma_{p}^{2}}-\frac{\left(\tilde{m}_{0}^{2}-m_{0}^{2}\right)^{2}}{8 \sigma_{m}^{2} E_{0}^{2}}\right) \rightarrow \pi^{8} \int \frac{d E}{\sqrt{2 \pi}} \delta^{(4)}\left(p-p_{P}\right) \delta^{(4)}\left(p-p_{D}\right),
$$

where $p=\left(E, \sqrt{E^{2}-\tilde{m}_{0}^{2}} \mathbf{l}\right)$, and with the approximations $p_{P}=p_{D}=p_{0}, v_{P}=v_{D}=0$, so that the energy and momentum decouple. The relationship is exact in the limit $\sigma_{p P}=$ $\sigma_{p D}=0$ (with the approximations for the momenta and velocities just mentioned).

These three rules lead to the macroscopic event rate at the detector at time $x_{D}^{0}$ :

$$
d \Gamma\left(x_{D}^{0}\right)=\int d \mathbf{x}_{P} \int d \mathbf{x}_{D} \int \frac{d \mathbf{Q}}{(2 \pi)^{3}} f\left(\mathbf{Q}, x_{P}\right) \int \frac{d \mathbf{Q}^{\prime}}{(2 \pi)^{3}} f\left(\mathbf{Q}^{\prime}, x_{D}\right) d \Gamma\left(\mathbf{Q}, \mathbf{Q}^{\prime}, \mathbf{x}_{P}, \mathbf{x}_{D}\right),
$$

with the constraint $x_{P}^{0}=x_{D}^{0}-L / v_{0}$. The single particle event rate is given by

$$
d \Gamma\left(\mathbf{Q}, \mathbf{Q}^{\prime}, \mathbf{x}_{P}, \mathbf{x}_{D}\right)=\int d E \text { flux } \times P_{\text {mix }} \times d \sigma .
$$

The flux is the flux of oscillating particles of energy $E$, produced at $x_{P}$ and detected at $x_{D}$. It includes a phase space factor for final state particles at production, $\int d \mathbf{K}$, as well as the delta function $\delta^{(4)}\left(p-p_{P}\right)$, the interaction vertex $\left|M_{P}(Q, K)\right|^{2}$, the geometrical factor $1 / L^{2}$, and a velocity factor $\left|v_{0}-v_{D}\right|$.

The factor $d \sigma$ is the cross section for the interaction of particle of mass $\tilde{m}_{0}$ in the detector. It includes a phase space factor for final state particles at production, $d \mathbf{K}^{\prime}$ (not integrated over 
if these momenta are measured), as well as the delta function $\delta^{(4)}\left(p-p_{D}\right)$, the interaction vertex $\left|M_{D}\left(Q^{\prime}, K^{\prime}\right)\right|^{2}$ and the Møller factor $\left|v_{0}-v_{D}\right|^{-1}$.

Finally, $P_{m i x}$ is identifiable as the flavor mixing transition probability:

$$
P_{\text {mix }}=\sum_{i, j} V_{i \alpha} V_{\beta i}^{-1} V_{j \alpha}^{*} V_{\beta j}^{-1 *} \exp \left(-2 \pi i \frac{L}{L_{i j}^{o s c}}-\frac{\left(\delta m_{i j}^{2}\right)^{2}}{32 p_{0}^{2}}\left(\frac{v_{0}^{2}}{\sigma_{m}^{2}}+\frac{\rho^{2}}{\sigma_{p e f f}^{2}}\right)-\left(\frac{L}{L_{i j}^{c o h}}\right)^{2}\right) .
$$

In comparison with $\mathcal{P}_{\alpha \rightarrow \beta}(\mathbf{L})$, given by Eq. (152), the geometrical decrease and the normalization factor, i.e. $N_{g} g^{2}(\mathbf{l}) / L^{2}$, are included in the flux and in $d \sigma$. The approximation $m_{0}=\left(m_{i}+m_{j}\right) / 2$ has also been made in each interference term.

In the case of a mixing of relativistic and nonrelativistic particles, the flavor-mixing probability does not factorize from the amplitudes of production and detection. The interference terms are however negligible because decoherence occurs. The dependence on the mass $m_{j}$ should be kept in the noninterference terms $\int d T\left|\mathcal{A}_{j}\right|^{2}$, with the result that the prefactor $v_{0}$ in (132) should be replaced by $v_{j}$. Velocity-dependent prefactors were already derived in the intermediate wave packet model of Giunti, Kim and Lee [141], except that the one-dimensional treatment in that article leads to a peculiar dependence in $v_{j}^{-1}$ (in our case, the conversion of the $1 / T^{2}$ prefactor into a $v_{j}^{2} / L^{2}$ prefactor yields an additional $v^{2}$, leading to the expected linear dependence in $v_{j}$ ). Cardall's correspondence rules are still valid, although the flux and the cross-section now depend on the mass eigenstate. For example, $\tilde{m}_{0}$ and $v_{0}$ are replaced respectively by $m_{j}$ and $v_{j}$ in the third rule. The single particle event rate is replaced by

$$
d \Gamma\left(\mathbf{Q}, \mathbf{Q}^{\prime}, \mathbf{x}_{P}, \mathbf{x}_{D}\right)=\sum_{j}\left|V_{j \alpha}\right|^{2}\left|V_{\beta j}^{-1}\right|^{2} \int d E \text { flux }_{j} \times d \sigma_{j} .
$$

Mixings of relativistic and nonrelativistic neutrinos have been studied by Ahluwalia and Goldman [8], who identify the third mass eigenstate with the $33.9 \mathrm{MeV}$ particle suggested by the KARMEN experiment [26]. Note that the amplitude of production (and also of detection) should be computed separately for the relativistic and nonrelativistic neutrinos.

\subsection{Coherence, incoherence and decoherence}

Generally speaking, decoherence is said to occur in particle oscillations if the interference terms in the transition probability are averaged to zero by some mechanism. In that case, the transition probability becomes independent of the distance. We have seen in section 7 that this phenomenon appears in the large-distance flavor-mixing transition probability (152) through the localization term and through the coherence length. Localization conditions, such as $L_{i j}^{o s c} \gtrsim \sigma_{x}$, determine whether there is decoherence from the start. If decoherence only occurs beyond a certain distance, the threshold is called the coherence length. These two phenomena are closely connected, since the coherence-length and localization conditions in the transversal-dispersion regime transform into the localization and coherence-length conditions in the longitudinaldispersion regime, respectively. In the end, both mechanisms of decoherence originate in the wave packets widths of the external particles. 
Other effects lead to similar constraints on the oscillations. They can be classified in coherent or incoherent effects. In quantum field theory, a coherent effect has to be taken into account in the amplitude, whereas an incoherent effect is incorporated into the computation only at the level of the probability [151]. Actually, this distinction has to be made only because approximations made in the computations often make the intrinsic decoherence effects disappear. For example, there are endless discussions about the energy and momentum coherence in the plane wave treatment of oscillations, since this approximation destroys all natural decoherence mechanisms. This should be contrasted with the fact that coherence-length and localization conditions appear explicitly in the oscillation formula (152) obtained in the external wave packet model. Moreover, decoherence between different energy components is automatic in the external wave packet model (see section 6.4). Note that one should be careful not to confuse the term '(in)coherent', referring here to a constraint applied to the amplitude or to the probability, with the term '(de)coherence', referring to the existence or disappearance of oscillations.

\subsubsection{Incoherent effects}

First, the energy-momentum spread of the beam has to be taken into account [37, 66, 34]. For example, the average of the oscillation term over a Gaussian momentum distribution of width $\Delta p$ and mean value $\bar{p}$ gives

$$
\int d p \exp \left(-2 \pi i \frac{L}{L_{i j}^{o s c}}-\frac{(p-\bar{p})^{2}}{2(\Delta p)^{2}}\right) \sim \exp \left(-2 \pi i \frac{L}{\bar{L}_{i j}^{o s c}}-2 \pi^{2}\left(\frac{\Delta p}{\bar{p}} \frac{L}{\bar{L}_{i j}^{o s c}}\right)^{2}\right),
$$

where $\bar{L}_{i j}^{o s c}=4 \pi \bar{p} / \delta m_{i j}^{2}$. A new coherence length can be defined by

$$
\bar{L}_{i j}^{c o h}=\frac{1}{\sqrt{2} \pi} \frac{\bar{p}}{\Delta p} \bar{L}_{i j}^{o s c} .
$$

This new coherence length can be obtained from the coherence length (154), derived in the external wave packet model, by substituting the beam spread $\Delta p$ for the effective width $\sigma_{p e f f}$. This result could be expected from the discussion of section 6.4, by extending the incoherent sum over the energy from a $\sigma_{\text {peff }}$ range to a $\Delta E=\Delta p / v_{0}$ range.

Second, the macroscopic propagation distance $L$ is not perfectly known [149, 66]. A Gaussian average over the macroscopic region of production gives

$$
\int d L \exp \left(-2 \pi i \frac{L}{L_{i j}^{o s c}}-\frac{(L-\bar{L})^{2}}{2(\Delta L)^{2}}\right) \sim \exp \left(-2 \pi i \frac{L}{L_{i j}^{o s c}}-2 \pi^{2}\left(\frac{\Delta L}{L_{i j}^{o s c}}\right)^{2}\right),
$$

where $\bar{L}$ is the average propagation distance and $\Delta L$ is the size of the source. Thus a new localization condition has to be satisfied:

$$
L_{i j}^{o s c} \gtrsim \Delta L
$$

This condition is similar to the localization condition (166) which comes from a coherent effect. The only thing to do is to substitute the size of the source $\Delta L$ for the effective width $\sigma_{x e f f}$. 
As noted several times ([104, 94, 237, 182, 248], and references therein), it is impossible, in stationary cases, to distinguish wave packets from an incoherent plane wave superposition with the same energy-momentum spectrum.

This observation leads directly to the generalization of the quantum field computation of the transition probability already done with external Gaussian wave packets. Arbitrary wave packets, with space width and momentum widths not minimizing the uncertainty, lead to an oscillation probability similar to Eq. (152) (or Eq. (187) if the particle is unstable), except that the relation $\sigma_{\text {xeff }} \sigma_{p e f f}=1 / 2$ does not hold anymore. This result can be understood as follows. If the arbitrary external wave packets are decomposed in Gaussian wave packets, the amplitude $\mathcal{A}_{i}$ becomes a superposition of Gaussian amplitudes. These do not interfere with each other if their phases are very different. Thus each Gaussian amplitude will mostly interfere with itself, from which an incoherent superposition follows.

In practice, the size of the region of production is usually much larger than the size of the wave packets. Similarly, the energy-momentum spread of the beam is usually much larger than the energy-momentum spread of the wave packet. Incoherent effects are thus very often dominant. At worst, the sizes of the regions of production and detection and of the energy-momentum spectrum are determined by the characteristics of the wave packet. Similar decoherence mechanisms were found by Gabor [131] in connection with electron-interference experiments. This author obtains three decoherence factors, coming from an average over the size of the source, from an average over the energy spectrum, and from the path difference between the interfering beams. The two first effects have been explained in this section, and the last effect can be related to the wave packet separation in particle oscillations.

\subsubsection{Coherent effects}

Recall that coherent effects are constraints on the oscillation process, which should be applied at the level of the amplitude. Most coherent effects can be expressed through their influence on the sizes of the external wave packets associated to the production and detection of the oscillating particle. Unfortunately, the estimate of a wave packet size is not an easy matter and no consensus exists on whichever evaluation method is the best.

Let us consider first a solar neutrinos. Only the cases of line spectra ( $p e p$ or ${ }^{7} \mathrm{Be}$ ) deserve careful thought, since the energy average always dominates coherent effects for continuum spectra (except if a detector with a extremely high energy resolution is invented). The most commonly discussed constraint on the wave packet size comes from the pressure broadening, that is, the interruption of coherent emission due to collisions of the emitting atoms. Nussinov [216], Loeb [200] and Kim and Pevsner [185] estimate the wave packet size of the parent nuclei at $\sigma_{x} \sim 10^{-6} \mathrm{~cm}$, whereas Krauss and Wilczek [188 propose $\sigma_{x} \sim 10^{-4} \mathrm{~cm}$. Kiers, Nussinov and Weiss [182] claim that the small wave packets of captured electrons give a stronger constraint $\sigma_{x} \sim 6 \times 10^{-8} \mathrm{~cm}$. However, none of these estimates gives a momentum width $\sigma_{p}$ as large as the $1 \mathrm{keV}$ energy spread of the solar neutrino line spectra [188, 221, 35]. The main contribution to this energy spread comes from the thermal energy spread of the captured electron [188], as well 
as from the Doppler shift due to the thermal motion of the emitting nucleus [221]. These effects cannot be modelized at the level of the amplitude and belong thus to incoherent effects. Finally, Malyshkin and Kulsrud [204] compute the effect of Coulomb collisions on the solar neutrino flux. They obtain a quantity $\sigma_{a}$, equivalent to the wave packet width, and consistent with Nussinov and Loeb's estimates. While Malyshkin and Kulsrud agree that pressure broadening can be neglected with respect to the line width, they claim that it could be relevant to the decoherence in the case of the continuous solar spectra, for a detector of very high resolution and a very long oscillation length. This conclusion is in contradiction with the increase of the coherence length with the resolution of the detector: the coherence length is mainly determined by the energy resolution of the detector, if it is smaller than the wave packet size at the source. All these results show that coherent effects seem to be irrelevant to solar neutrinos.

As regards neutrinos from supernovae, the source wave packet width has been estimated by Anada and Nishimura at $\sigma_{x} \sim 10^{-14} \mathrm{~cm}$ for neutrinos from the supernova core [19], and at $\sigma_{x} \sim 10^{-9} \mathrm{~cm}$ for neutrinos from the neutrino sphere [20]. Since $p \sim 10 \mathrm{MeV}$, core neutrinos decohere before oscillating $\left(p / \sigma_{p} \sim 0.1\right)$, whereas sphere neutrinos might oscillate if the oscillation length is not too short $\left(p / \sigma_{p} \sim 10^{4}\right)$. However, the incoherent momentum spread $(p / \Delta p \sim 1-10)$ destroys any interference effect left, unless the oscillation length is, by chance, comparable to the supernova-Earth distance [236].

Another type of neutrino source is a radioactive nucleus in an atomic lattice, which is the case for reactor neutrinos. The wave packet size can be estimated by Rich [237] and Grimus and Stockinger [150] at $\sigma_{x} \sim 10^{-10} \mathrm{~m}$. The wave packet size of the emitted electrons is larger and can be neglected [185]. With the neutrino energy around $1 \mathrm{MeV}$, decoherence occurs beyond $\frac{p}{\sigma_{p}} \sim 10^{3}$ oscillation lengths, so that there are no coherent effects relevant for laboratory experiments. Of course, oscillations may vanish because of the incoherent energy average.

Coherent effects may also appear because of the finite lifetime of the source $\tau_{\text {decay }}$, which interrupts the classical emission of the wave train and limits the size of the wave packet to $\sigma_{x} \sim c \tau_{\text {decay }}$ [182]. This effect could be relevant to atmospheric neutrinos and in accelerators. For quasi-stable sources, the constraint $L_{i j}^{o s c} \gtrsim \sigma_{x}$ can be very stringent. For example, in the case of $\pi \rightarrow \mu \nu$, the length of the wave packet is bounded by $c \tau_{\text {decay }} \sim 7.8 \mathrm{~m}$ and could be macroscopically large. However this argument is only valid as long as the decay point of the source is not observed at all. As emphasized by Kayser [176], the detection of a final state, such as the muon in $\pi \rightarrow \mu \nu$, can localize the decay point to a precision much better than either $c \tau_{\text {decay }}$ or the macroscopic size of the production region. An extreme example is given by the neutrinos from the $\beta$ decay of a nucleus with $\tau_{\text {decay }} \sim 1 \mathrm{sec}$. The observation of the $\beta$ particle allows to pin down the decay point to a precision much better than either $c \tau_{\text {decay }} \sim 10^{5} \mathrm{~km}$ or the production region, for example a nuclear reactor. Thus the relation between the decay time of the source and the wave packet size of the oscillating particle is not direct. The decay time only puts an upper bound to the wave packet length. Some papers dealing with the finite lifetime of the source are reviewed in section 9.3 .

Since the coherence length depends not only on the characteristics of the source, but also 
on those of the detector, the minimal wave packet size in the detection process must also be checked. It can be roughly estimated at $\sigma_{x} \sim 10^{-10} \mathrm{~m}$, that is, $\sigma_{p} \sim 10^{-3} \mathrm{MeV}$ [151]. In all cases, this momentum width is not larger than the energy spread and can be neglected.

As regards $\mathrm{K}$ and $\mathrm{B}$ mesons, particle decay takes place before decoherence occurs:

$$
\frac{L_{i j}^{c o h}}{L_{i j}^{\text {decay }}} \sim \frac{p_{0}}{\sigma_{p e f f}} \frac{\Gamma_{i}+\Gamma_{j}}{2\left|m_{i}-m_{j}\right|} \sim \frac{p_{0}}{\sigma_{p e f f}} \gg 1,
$$

where $L_{i j}^{c o h}$ and $L_{i j}^{\text {decay }}$ are given by Eqs. (154) and (186), respectively.

To sum up, coherent effects are most likely irrelevant with respect to incoherent effects. This discussion also shows that, in practice, we are far from being able to increase the coherence length by more accurate energy measurements.

\section{$9.3 \quad$ Unstable source}

In principle, the instability of the source can be taken into account in a field-theoretical model by considering the source as another internal line of the global Feynman diagram describing the process. The difficulty, of technical nature, consists in integrating on both propagators, with a constraint on the decay point which can be either nonexistent, or very stringent.

The case of an unstable source decaying in flight has been considered by Campagne in a field-theoretical model [84]. Besides the usual condition $L_{i j}^{o s c} \gtrsim \sigma_{x P, D}$, this author obtains a new localization condition $L_{i j}^{o s c} \gtrsim L_{P}^{\text {decay }}$, where $L_{P}^{\text {decay }}$ is either the decay length $p / m \Gamma$ of the unstable source, or the length of the decay tunnel, if the latter is shorter. However, this treatment is not a real improvement on the one proposed by Rich, who had derived earlier the same results in the framework of time perturbation theory in quantum mechanics 237. It is not satisfactory that macroscopic quantities, such as the lengths of the source, of the target and of the pion decay tunnel, are treated in [84] on the same footing as microscopic quantities.

The case of an unstable source at rest has been considered in detail by Grimus, Mohanty and Stockinger 151, 152]. They use their previous field-theoretical model [150, modified by a quantum-mechanical Wigner-Weisskopf approximation, in order to take into account the finite lifetime of the source. Besides the already known condition $L_{i j}^{o s c} \gtrsim \sigma_{x P, D}$, they obtain a new localization condition:

$$
\frac{\sigma_{P}}{m_{P} \Gamma_{P}} \lesssim \frac{1}{4 \pi} L_{i j}^{o s c}
$$

where $\sigma_{P}, m_{P}$ and $\Gamma_{P}$ are respectively the wave packet momentum width, the mass, and the decay width of the unstable parent particle. In brief, the unstable source should not move on distances larger than the oscillation length during its lifetime, or else the oscillations vanish. They find that this condition is most likely satisfied in the experiments LSND [28, 29] and KARMEN [26, 27]. The same authors have also found a new coherence length, $L_{i j}^{c o h}=4 E^{2} / \delta m_{i j}^{2} \Gamma_{P}$, which is however completely irrelevant, since it is much larger than the coherence lengths discussed previously ( $\Gamma_{P} \ll \sigma_{P}$ for all weakly unstable particles). The widths $\sigma_{x P, D}$ can be estimated at $10^{-2} \mathrm{MeV}$ for LSND and KARMEN [152], so that the condition $L_{i j}^{o s c} \gtrsim \sigma_{x P, D}$ is also satisfied. 
A slightly different model for $\pi \rightarrow \mu \nu$ is proposed by Dolgov 105]. This author computes the transition amplitude by coupling the neutrino propagator to external wave packets at the source, but not at the detector (source-propagator model). The finite lifetime of the source is taken into account with a Wigner-Weisskopf approximation, like in the articles discussed above [151, 152], but without the restriction of a source at rest. Dolgov considers first the case where the decay point of the muon is not registered (but its energy-momentum is perfectly known) and computes an amplitude of spatial width $\gamma=p_{\pi} / m_{\pi} \Gamma_{\pi}$. The oscillating phase is given by

$$
\phi_{i j}(T, L)=2 \pi \frac{L}{L_{i j}^{o s c}}+\alpha \frac{L-v_{0} T}{L_{i j}^{o s c}} \quad \text { with } \quad \alpha=2 \pi \frac{\mathbf{v}_{\pi} \cdot \mathbf{v}_{0}}{\mathbf{v}_{0}^{2}-\mathbf{v}_{\pi} \cdot \mathbf{v}_{0}}
$$

where $\mathbf{v}_{\pi}$ and $\mathbf{v}_{0}$ are the velocities of the pion and neutrino, respectively. With the help of the constraint $0 \lesssim v_{0} T-L \lesssim \gamma$, the second term of the phase is seen to be negligible if $L_{i j}^{o s c} \gg \alpha \gamma$. For a source at rest, $\alpha=0$ and this localization condition vanishes. When the muon decay point is registered, Dolgov obtains an oscillation phase equal to the standard result as long as the localization condition $\sigma_{\pi} \ll L_{i j}^{o s c}$ is satisfied (where $\sigma_{\pi}$ is the size of the wave packet of the initial pion). It would be interesting to study intermediate situations where the muon is registered with a space-time uncertainty and to compute the time-independent oscillation probability.

This brief account of the treatment of oscillations from an unstable source shows that there is still work to do, especially regarding decays in flight.

\section{Correlated oscillations}

\subsection{Introduction}

Experiments where two correlated mesons oscillate together are very interesting for the study of $\mathrm{CP}$ violation [82, 83, 77] and can provide tests of the Einstein-Podolsky-Rosen (EPR) effect [25, 57, 58, 116, 228]. At the present time, the process $\phi(1020) \rightarrow K^{0} \overline{K^{0}}$ is studied by the experiment DA $\Phi$ NE at Frascati [7]. The process $\Upsilon(4 s) \rightarrow B^{0} \overline{B^{0}}$ is studied at $B$ factories such as KEKB at Tsukuba [4] or BABAR at Stanford [31, 33], where asymmetric collisions allow the measurement of the $B$ pathlengths. In principle, correlated oscillations could occur with particles not conjugated to each other, for example the lepton could oscillate between $e$ and $\mu$ and the neutrino between $\nu_{e}$ and $\nu_{\mu}$ in the process $\pi \rightarrow l \nu$. However, the charged lepton masses are too different for such oscillations to be observable (but note that if the masses were close enough to allow oscillations, it would be impossible to identify the flavor).

In this section, we develop the formalism appropriate for correlated oscillations and we apply it to oscillations of correlated mesons. We then disprove a claim of a non-standard oscillation length. The question of the oscillation of recoil particles, like $\Lambda$ in $\pi p \rightarrow \Lambda K$ or $\mu$ in $\pi \rightarrow \mu \nu$, can be examined in the same framework, allowing us to answer the fifth question posed in section 3.2 . 
The treatment of one-particle oscillations with the external wave packet model of section can be easily extended by associating a propagator with each oscillating particle. Consider the two successive processes:

$$
\begin{aligned}
\left(e^{+} e^{-}\right)\left(p_{P}\right) & \rightarrow \mathcal{R} X \\
\mathcal{R} & \rightarrow P_{1} P_{2} \rightarrow f_{1}\left(p_{D_{1}}\right) f_{2}\left(p_{D_{2}}\right)
\end{aligned}
$$

where $p_{P}$ and $p_{D_{1,2}}$ are the average momenta of the source $\mathcal{R}$ and of the final states, respectively. We would like to apply the external wave packet model to the second process. In principle this model should be modified to include the decay width of the source $\mathcal{R}$, since one external particle at an interaction point is not sufficient to localize it. However the decay widths of the $\phi(1020)$ and $\Upsilon(4 s)$ are large, so that their decay point will be close to their production point. The latter can be localized with the $e^{ \pm}$external wave packets. In the case of a small decay width, it will be seen below that the correlated oscillations become independent of the production point.

Note that the initial flavors of the oscillating particles cannot be observed at the source and must be summed over. For example, $B^{0} \bar{B}^{0}$ and $\bar{B}^{0} B^{0}$ are both produced in the $\Upsilon(4 s)$ decay. In contradistinction to the one-particle oscillation case, this sum does not destroy the oscillations, because of the flavor correlation between the oscillating particles. Indeed, two mesons of the same flavor cannot be observed in the above process at the same time in the center of mass frame (EPR effect).

Let us first write the amplitude corresponding to the process (199). The masses of the first particle are noted $m_{1 a}, m_{1 b}$ etc., while the masses of the second particle are noted $m_{2 i}, m_{2 j}$ etc. The two sets coincide if $P_{1}$ and $P_{2}$ are conjugated mesons, but it is not necessarily the case. The average production and detection points are noted $x_{P}$ and $y_{D_{1}}, y_{D_{2}}$, respectively. The partial amplitude, corresponding to the propagation of the eigenstates of mass $m_{1 a}$, from $x_{P}$ to $y_{D_{1}}$, and of mass $m_{2 i}$, from $x_{P}$ to $y_{D_{2}}$, can be written as

$$
\mathcal{A}_{a, i}=\int d^{4} p_{1} \int d^{4} p_{2} \psi\left(p_{1}, p_{2}\right) G_{1 a}\left(p_{1}^{2}\right) G_{2 i}\left(p_{2}^{2}\right) e^{-i p_{1} \cdot\left(y_{D_{1}}-x_{P}\right)-i p_{2} \cdot\left(y_{D_{2}}-x_{P}\right)},
$$

with the overlap function given by

$$
\psi\left(p_{1}, p_{2}\right)=N \psi_{P}\left(p_{1}^{0}+p_{2}^{0}, \mathbf{p}_{1}+\mathbf{p}_{2}\right) \psi_{D_{1}}\left(p_{1}^{0}, \mathbf{p}_{1}\right) \psi_{D_{2}}\left(p_{2}^{0}, \mathbf{p}_{2}\right)
$$

The production overlap function $\psi_{P}$ is defined by Eq. (54). The detection overlap function $\psi_{D_{1}}$ is defined by the same equation, where $\mathbf{p}_{D_{1}}, E_{D_{1}}$ and $\mathbf{v}_{D_{1}}$ have been substituted to $\mathbf{p}_{P}, E_{P}$ and $\mathbf{v}_{P}$, respectively. The definition of $\psi_{D_{2}}$ is similar.

The integration on the energies $p_{1,2}^{0}$ can be done with the help of the Jacob-Sachs theorem (211), yielding

$$
\mathcal{A}_{a, i} \sim \int d^{3} p_{1} \int d^{3} p_{2} \psi\left(z_{1}, z_{2}, \mathbf{p}_{1}, \mathbf{p}_{2}\right) e^{-i \sqrt{z_{1}+\mathbf{p}_{1}^{2}} T_{1}+i \mathbf{p}_{1} \cdot \mathbf{L}_{1}} e^{-i \sqrt{z_{2}+\mathbf{p}_{2}^{2}} T_{2}+i \mathbf{p}_{2} \cdot \mathbf{L}_{2}},
$$

where $T_{1,2}=t_{D_{1,2}}-t_{P}, \mathbf{L}_{1,2}=\mathbf{y}_{D_{1,2}}-\mathbf{x}_{P}$ are the average propagation time and distance of $P_{i}$, and $z_{i}$ is the pole of the propagator $G_{i}\left(p^{2}\right)$. 
Since our aim is not to prove the conservation of energy-momentum between the initial state $\phi(1020)$ and the final states $f_{1,2}$, we set

$$
\mathbf{p}_{D_{1}}+\mathbf{p}_{D_{2}}=\mathbf{p}_{P} \quad \text { and } \quad E_{D_{1}}+E_{D_{2}}=E_{P}
$$

As before, it will be useful to define reference masses $m_{1}$ and $m_{2}$ through $m_{1,2}^{2}=E_{D_{1,2}}^{2}-\mathbf{p}_{D_{1,2}}^{2}$. The velocities $\mathbf{v}_{1,2}$ are defined by $\mathbf{v}_{1,2}=\mathbf{p}_{D_{1,2}} / E_{D_{1,2}}$ (they should not be confused with $\mathbf{v}_{D_{1,2}}$, which appear in the overlap function and refer to the velocities of the detection regions, as explained in section 4.5.2).

\subsection{Factorization}

The momentum integrations in the amplitude (200) can be easily evaluated, either with Laplace's method or with the stationary phase method, provided that the energy-momentum correlation is not too stringent at the source. More precisely, the integrations on $\mathbf{p}_{1}$ and $\mathbf{p}_{2}$ can be done independently if the energy uncertainty at the source is larger than the energy uncertainties at the detection points (recall that the momentum width is always larger than the energy width). For example, the production widths for the $\phi(1020)$ decay and the $\Upsilon(4 s)$ decay can be estimated by their average decay widths, which are $4.26 \mathrm{MeV}$ and $14 \mathrm{MeV}$, respectively [156]. Both widths are larger than typical detector uncertainties. In that case, the energy-momentum width at the source can be neglected and the overlap function factorizes in one-particle overlap functions:

$$
\psi\left(z_{1}, z_{2}, \mathbf{p}_{1}, \mathbf{p}_{2}\right) \cong \psi_{1}\left(z_{1}, \mathbf{p}_{1}\right) \psi_{2}\left(z_{2}, \mathbf{p}_{2}\right)
$$

Thus the resulting amplitude factorizes in one-particle oscillation amplitudes. The rest of the computation proceeds as in the one-particle case, except when the detection times are measured: the time average is then done only once, on the production time.

If the energy-momentum uncertainty is smaller at the production point than at the detection points, energy-momentum correlations are introduced at the source so that the amplitude cannot be factorized. However, the oscillation formula is not expected to be modified, as long as the energy-momentum uncertainty at the source is larger than the mass difference between the interfering mass eigenstates: $\sigma_{p P} \gtrsim \delta m_{i j}^{2} / p_{D_{1,2}}$. This condition resembles the localization condition derived in the one-particle oscillation case, which stated that oscillations vanish if the uncertainty on the position of the source is larger than the oscillation length. This similarity is

misleading: it will be seen correlated oscillations do not vanish if $\sigma_{p P} \lesssim \delta m_{i j}^{2} / p_{D_{1,2}}$, since the knowledge of the source energy-momentum is not sufficient to ascertain which mass eigenstates are produced. This is because the energy-momentum of the source is shared between two intermediate particles, instead of one as in the one-particle oscillation model presented in section 4 . Moreover, the position and time of the production process can be reconstructed, in principle, from the final states characteristics, so that the oscillation pattern is not washed out by an illdefined production point. The discussion of the above constraint is somewhat academic, as it is satisfied for the experimentally studied processes, $\phi \rightarrow K^{0} \overline{K^{0}}$ and $\Upsilon(4 s) \rightarrow B^{0} \overline{B^{0}}$. Nevertheless, 
the question of whether the violation of this constraint changes the oscillation formula will be examined, partly as a matter of principle, and partly because it will be useful for the discussion of the recoil oscillation conundrum.

\subsection{Energy-momentum correlation at the source}

There are two reasons to study more carefully energy-momentum correlations at the source. First, this analysis yields a correlated localization condition, arising from the source. In particular, it allows to check explicitly that a zero momentum width at the source does not wash out the oscillations. Second, it will be useful for the examination of the so-called recoil oscillations. Unfortunately the treatment of the full 3-dimensional case is involved, because the correlation at the source might link a variation in the longitudinal momentum of one particle, with a variation in the transversal momentum of the other. If the transversal dispersion is neglected, the integrals can in principle be evaluated with Laplace's method in three dimensions, but the results are lengthy. For that reason, only the collinear case will be presented. Momenta, velocities and lengths can take positive or negative values, with the sign indicating the direction.

Since the stationary phase and Laplace's method give the same results (see section 6), we choose the latter for the longitudinal momenta integrations. Besides, the dispersion will be neglected. Recall that the masses of the first particle are noted $m_{1 a}, m_{1 b}$ etc., while the masses of the second particle are noted $m_{2 i}, m_{2 j}$ etc. Suppose that the overlap function $\psi$ is maximal for $p_{1}=p_{1 a}$ and $p_{2}=p_{2 i}$. As before, these momenta can be computed by expanding the argument of the overlap function in small mass differences. The expansion parameters are given by $\delta m_{1 a}^{2}=m_{1 a}^{2}-m_{1}^{2}$ and $\delta m_{2 i}^{2}=m_{2 i}^{2}-m_{2}^{2}$. At first order in mass differences, the average momenta of the particles are

$$
\begin{aligned}
& p_{1 a}=p_{D_{1}}+\frac{\sigma_{x e f f 3}^{2} c_{2}-\sigma_{x e f f 2}^{2} c_{1}}{4 \Delta}, \\
& p_{2 i}=p_{D_{2}}+\frac{\sigma_{x e f f 3}^{2} c_{1}-\sigma_{x e f f 1}^{2} c_{2}}{4 \Delta},
\end{aligned}
$$

where $\Delta=\sigma_{x e f f 1}^{2} \sigma_{x e f f 2}^{2}-\sigma_{x e f f 3}^{4} \geq 0$. In momentum space, the effective widths read

$$
\begin{aligned}
\frac{1}{\sigma_{p e f f 1,2}^{2}} & =\frac{1}{\sigma_{p P}^{2}}+\frac{\left(v_{1,2}-v_{P}\right)^{2}}{\sigma_{e P}^{2}}+\frac{1}{\sigma_{p_{D_{1}, 2}}^{2}}+\frac{\left(v_{1,2}-v_{D_{1,2}}\right)^{2}}{\sigma_{e D_{1,2}}^{2}} \\
\frac{1}{\sigma_{p e f f 3}^{2}} & =\frac{1}{\sigma_{p P}^{2}}+\frac{\left(v_{1}-v_{P}\right)\left(v_{2}-v_{P}\right)}{\sigma_{e P}^{2}}
\end{aligned}
$$

They are related to the effective widths in configuration space by $\sigma_{p e f f 1,2} \sigma_{x e f f 1,2}=1 / 2$. Their name is justified below by their appearance as second order coefficients in the expansion of the overlap function. These expressions can be compared to the effective width (89) in the one-particle oscillation case. The mass differences are included in the constants $c_{k}$ :

$$
c_{k}=\frac{v_{k}-v_{D_{k}}}{\sigma_{e D_{k}}^{2}} \delta_{k}+\frac{v_{k}-v_{P}}{\sigma_{e P}^{2}}\left(\delta_{1}+\delta_{2}\right),
$$


with $\delta_{1}=\delta m_{1 a}^{2} / 2 E_{D_{1}}$ and $\delta_{2}=\delta m_{2 i}^{2} / 2 E_{D_{2}}$.

If the overlap function is noted $\psi=N \exp \left(-f\left(p_{1}, p_{2}\right)\right)$, the expansion of the argument of the exponential around its extremum can be written as

$$
f\left(p_{1}, p_{2}\right)=f\left(p_{1 a}, p_{2 i}\right)+\frac{\left(p_{1}-p_{1 a}\right)^{2}}{4 \sigma_{p e f f 1}^{2}}+\frac{\left(p_{2}-p_{2 i}\right)^{2}}{4 \sigma_{p e f f 2}^{2}}+\frac{\left(p_{1}-p_{1 a}\right)\left(p_{2}-p_{2 i}\right)}{2 \sigma_{p e f f 3}^{2}} .
$$

The evaluation in one dimension of the integral (200) by Laplace's method yields

$$
\begin{aligned}
& \mathcal{A}_{a, i} \sim \exp \left(-f\left(p_{1 a}, p_{2 i}\right)\right) \exp \left(-i E_{1 a} T_{1}+i p_{1 a} L_{1}-\frac{m_{1} \Gamma_{1} T_{1}}{2 E_{1 a}}-i E_{2 i} T_{1}+i p_{2 i} L_{1}-\frac{m_{2} \Gamma_{2} T_{2}}{2 E_{2 i}}\right) \\
& \times \exp \frac{-1}{4 \Delta}\left(\sigma_{x e f f 2}^{2}\left(v_{1 a} T_{1}-L_{1}\right)^{2}+\sigma_{x e f f 1}^{2}\left(v_{2 i} T_{2}-L_{2}\right)^{2}-2 \sigma_{x e f f 3}^{2}\left(v_{1 a} T_{1}-L_{1}\right)\left(v_{2 i} T_{2}-L_{2}\right)\right),
\end{aligned}
$$

where $v_{1 a}=p_{1 a} / E_{1 a}$, with $E_{1 a}=\sqrt{p_{1 a}^{2}+m_{a}^{2}}$. The velocity $v_{2 i}$ and the energy $E_{2 i}$ are similarly defined. Once more 'wave packets' can be associated with the oscillating particles 1 and 2, but they are correlated by the finite width $\sigma_{x e f f 3}$. The phase can be expanded around $p_{D_{1,2}}$ and $m_{1,2}$. For example,

$$
\phi_{1 a}=E_{1 a} T_{1}-p_{1 a} L_{1} \cong E_{D_{1}} T_{1}-p_{D_{1}} L_{1}+\left(v_{1} T_{1}-L_{1}\right)\left(\delta p_{1 a}+\frac{\delta m_{1 a}^{2}}{2 p_{1}}\right)+\frac{\delta m_{1 a}^{2}}{2 p_{1}} L_{1},
$$

where $\delta p_{1 a}=p_{1 a}-p_{1}$. The phase difference between two amplitudes vanishes but for the oscillation term already encountered in the previous sections, provided the wave packet is sufficiently localized, so that the term proportional to $v_{1} T_{1}-L_{1}$ in (206) is negligible.

At the extremum, the argument of the overlap function reads

$$
f\left(p_{1 a}, p_{2 i}\right)=\frac{\alpha \delta_{1}^{2}+\beta \delta_{2}^{2}+2 \gamma \delta_{1} \delta_{2}}{64 \Delta}
$$

and yields a localization condition, that is, the mass differences cannot be too large compared with the energy-momentum widths. The coefficients $\alpha$ and $\gamma$ read

$$
\begin{aligned}
\alpha & =\frac{1}{\sigma_{p P}^{2} \sigma_{e P}^{2}}\left(\frac{1}{\sigma_{D}^{2}}+\frac{\left(v_{2}-v_{D_{1}}\right)^{2}}{\sigma_{e D_{1}}^{2}}+\frac{\left(v_{2}-v_{D_{2}}\right)^{2}}{\sigma_{e D_{2}}^{2}}\right) \\
& +\left(\frac{1}{\sigma_{p D_{2}}^{2}}+\frac{\left(v_{2}-v_{D_{2}}\right)^{2}}{\sigma_{e D_{2}}^{2}}\right)\left(\frac{1}{\sigma_{p P}^{2} \sigma_{e D_{1}}^{2}}+\frac{1}{\sigma_{e P}^{2} \sigma_{p D_{1}}^{2}}+\frac{\left(v_{P}-v_{D_{1}}\right)^{2}}{\sigma_{e P}^{2} \sigma_{e D_{1}}^{2}}\right)+\frac{1}{\sigma_{p D_{1}}^{2} \sigma_{e D_{1}}^{2} \sigma_{p e f f 2}^{2}}, \\
\gamma & =\frac{1}{\sigma_{p P}^{2} \sigma_{e P}^{2}}\left(\frac{1}{\sigma_{D}^{2}}+\frac{\left(v_{1}-v_{D_{1}}\right)\left(v_{2}-v_{D_{1}}\right)}{\sigma_{e D_{1}}^{2}}+\frac{\left(v_{2}-v_{D_{2}}\right)\left(v_{1}-v_{D_{2}}\right)}{\sigma_{e D_{2}}^{2}}\right)+\frac{\left(v_{1}-v_{D_{1}}\right)\left(v_{2}-v_{D_{2}}\right)}{\sigma_{p P}^{2} \sigma_{e D_{1}}^{2} \sigma_{e D_{2}}^{2}} \\
& +\frac{1}{\sigma_{e P}^{2}}\left(\frac{1}{\sigma_{p_{D_{1}}}^{2}}+\frac{\left(v_{1}-v_{D_{1}}\right)\left(v_{P}-v_{D_{1}}\right)}{\sigma_{e D_{1}}^{2}}\right)\left(\frac{1}{\sigma_{p_{D_{2}}}^{2}}+\frac{\left(v_{2}-v_{D_{2}}\right)\left(v_{P}-v_{D_{2}}\right)}{\sigma_{e D_{2}}^{2}}\right),
\end{aligned}
$$

where $\sigma_{p D}^{-2}=\sigma_{p D_{1}}^{-2}+\sigma_{p D_{2}}^{-2}$. The coefficient $\beta$ is obtained from $\alpha$ by the exchange of the indices $1 \leftrightarrow 2$.

What happens when the energy-momentum uncertainty at the source goes to zero? Three features of the amplitude (205) must be examined: the localization condition (207) given by the 
function $f\left(p_{1 a}, p_{2 i}\right)$, the value of the phase and the 'wave packet' effect on the time average. In the limit $\sigma_{p P} \rightarrow 0$ (so that $\sigma_{e P} \rightarrow 0$ too), the argument of the overlap function becomes at the extremum

$$
\lim _{\sigma_{p P} \rightarrow 0} f\left(p_{1 a}, p_{2 i}\right)=\frac{\left(\left(v_{2}-v_{D_{1}}\right) \delta_{1}+\left(v_{1}-v_{D_{1}}\right) \delta_{2}\right)^{2}}{4\left(v_{1}-v_{2}\right)^{2} \sigma_{e D_{1}}^{2}}+\frac{\left(\left(v_{2}-v_{D_{2}}\right) \delta_{1}+\left(v_{1}-v_{D_{2}}\right) \delta_{2}\right)^{2}}{4\left(v_{1}-v_{2}\right)^{2} \sigma_{e D_{2}}^{2}}+\frac{\left(\delta_{1}+\delta_{2}\right)^{2}}{4\left(v_{1}-v_{2}\right)^{2} \sigma_{p D}^{2}}
$$

Thus a small, or even zero energy-momentum uncertainty at the source, does not destroy the oscillations. This result confirms our expectations, since a zero width at the source does not give information on which mass eigenstates propagate. On the other hand, the function $f\left(p_{1 a}, p_{2 i}\right)$ diverges when either $\sigma_{p_{D_{1}}}^{2}$ or $\sigma_{p_{D_{2}}}^{2}$ goes to zero, yielding the expected localization conditions at the detectors.

In the limit of a zero energy-momentum width at the source, wave packets associated to oscillating particles are of infinite extent. This phenomenon was expected, since the time of production $T_{P}$, included in $T_{1}$ and $T_{2}$, becomes ill-defined. However the average on the time of production does not destroy the oscillations, at least if the detection times are measured. The explanation follows. In the limit $\sigma_{p P} \rightarrow 0$, Eqs. (202) and (203) show that the quantities $\delta p_{1 a}=p_{1 a}-p_{D_{1}}$ and $\delta p_{2 i}=p_{2 i}-p_{D_{2}}$ become

$$
\lim _{\sigma_{p P} \rightarrow 0} \delta p_{1 a}=-\lim _{\sigma_{p P} \rightarrow 0} \delta p_{2 i}=-\frac{\delta_{1}+\delta_{2}}{v_{1}-v_{2}} .
$$

Note that these values can be directly obtained, in the one-dimensional case, from energymomentum conservation at the source. The phase of the amplitude (see Eq. 206) ) can then be written as

$$
\lim _{\sigma_{p P} \rightarrow 0}\left(\phi_{1 a}+\phi_{2 i}\right)=\delta_{1} \frac{L_{1}-L_{2}-v_{2}\left(T_{1}-T_{2}\right)}{v_{1}-v_{2}}+\delta_{2} \frac{L_{1}-L_{2}-v_{1}\left(T_{1}-T_{2}\right)}{v_{1}-v_{2}},
$$

where the term of order zero in the mass differences has been dropped. Since $T_{1}-T_{2}=t_{D_{1}}-t_{D_{2}}$ and $L_{1}-L_{2}=y_{D_{1}}-y_{D_{2}}$, the phase is independent of the production point. However the production point is implicit in the phase (208) when the classical limit is taken. The substitution of the classical relations $L_{1,2}=v_{1,2} T_{1,2}$ shows indeed that the expressions multiplying $\delta_{1}$ and $\delta_{2}$ are the propagation times $T_{1}$ and $T_{2}$, respectively. In other words the space-time coordinates of the production point can be reconstructed from the coordinates of detection and the velocities of the oscillating particles, with the exception of the $v_{1}=v_{2}$ case (recall that velocities of the same sign have the same direction). While complete computations in three dimensions are complicated, it can be seen that the phase difference becomes independent of the production coordinates, in the limit of zero energy-momentum uncertainty at production:

$$
\lim _{\sigma_{p P} \rightarrow 0}\left(\phi_{1 a}+\phi_{2 i}-\phi_{1 b}-\phi_{2 j}\right)=\delta E_{1 a b}\left(T_{1}-T_{2}\right)-\delta \mathbf{p}_{1 a b} \cdot\left(\mathbf{L}_{1}-\mathbf{L}_{2}\right) .
$$

\subsection{No recoil oscillations}

It has been claimed that particles produced together with mixed states also oscillate because of the energy-momentum recoil. For example, the $\Lambda$ baryon, in the process $\pi^{-} p \rightarrow \Lambda K^{0}$, is 
supposed to be in a superposition of two energy-momentum eigenstates, so that its detection probability should oscillate in space [244]. Furthermore the oscillation frequency of the kaon is modified by the momentum recoil against the $\Lambda$. In another example, muons produced in $\pi \rightarrow \mu \nu$ are supposed to oscillate in space, because of the momentum recoil against the mixed state of the neutrino 246, 247. As explained in section 2.3, recoil oscillations arise in quantummechanical models if different propagation times are associated to the different mass eigenstates. Recoil oscillations are unacceptable from basic principles, since the oscillation of the detection probability of a non-mixed state means that probability is not conserved at all distances. On the contrary, when several mass eigenstates are mixed, the sum of the detection probabilities of the different mass eigenstates is always equal to 1 for a given propagation distance.

This assertion has been questioned in several papers in the framework of quantum mechanics. These refutations involve either the consideration of the different proper times associated with the oscillating particle and the recoil particle [201, 258, 80], or the use of classical trajectories [107], or the use of energy-momentum conservation for the average energy-momenta of the propagating wave packets [258, 212]. The source-propagator approach of Shtanov [242] is not a real improvement on the arguments of Ref. [107], since Shtanov treats configuration space variables, which have a microscopic role, as classical macroscopic variables.

Quantum field theory allows to understand which quantum-mechanical explanations are correct and important. The correlated oscillation model of the previous section is easily adapted to the treatment of recoil oscillations. Suppose that the first particle is in a superposition of several mass eigenstates $m_{1 a}$, whereas the second particle has only one mass eigenstate $m_{2}$ (it is the so-called recoil particle). Since the phase of the amplitude (205) depends only on the average propagation times $T_{1,2}$ and distances $L_{1,2}$, which are common to the different mass eigenstates, no recoil oscillations will be observed as long as these quantities are well-defined. This can be checked explicitly if $\sigma_{p P} \gtrsim \sigma_{p D_{1,2}}$, as the overlap function factorizes in that case. Thus the only case requiring a careful examination is when the uncertainty on the position of the source is larger than the oscillation length, i.e. when $\sigma_{p P} \lesssim \delta m_{1 a b}^{2} / 2 p_{1}$. This condition is not satisfied in the process $\pi^{-} p \rightarrow \Lambda K^{0}$. The momentum uncertainty of the proton, in this fixed-target experiment, is around $3 \mathrm{keV}$, i.e. much larger than the kaon mass difference of $3 \times 10^{-9} \mathrm{keV}$ [198]. Therefore the problem of recoil oscillations does not arise in the $\Lambda K^{0}$ complex.

In contradistinction to the case of the $\pi^{-} p$ collision, it is possible to consider a $\pi$ decay with a sharp momentum. Its theoretical minimal uncertainty is given by its $2.5 \times 10^{-8} \mathrm{eV}$ decay width, which is smaller than the neutrino mass differences. This case has been treated, with essentially correct quantum-mechanical arguments, by Dolgov, Morozov, Okun and Schepkin [107] and within a model coupling the $\mu$ and $\nu$ propagators with a source wave packet by Dolgov [105]. On the one hand, if only the neutrino is observed, the detailed study of the oneparticle oscillation case in section 6 has shown that neutrino oscillations occur, provided that the momentum width associated with the muon is larger than the inverse oscillation length. On the other hand, if only the muon is observed, no oscillations in the probability of muon detection occur. The reason is that the sum over the neutrino flavors makes the transition probability 
(152) independent of the distance (apart from the geometrical decrease in $L^{-2}$ ):

$$
\sum_{\alpha} \int L^{2} d \Omega \mathcal{P}_{\alpha \rightarrow \beta}(\mathbf{L})=1
$$

Furthermore oscillations also vanish after integration over the unknown source decay coordinates. This point was discussed in section 9.3 .

Finally, the only case left is the detection of both muon and neutrino, coming from the decay of a pion endowed with a sharp momentum. As explained above, this process can be treated like a correlated oscillation, but with only one mass eigenstate $m_{2}$ for the muon. The formula (209) shows that the oscillation of the probability is independent of the production point, so that the average over the production point does not have any effect on the phase. Moreover the oscillation term can be expressed as depending only on the detection point of the neutrino, and on the reconstructed decay point (see Eq. (208) with $\delta_{2}=0$ ):

$$
\lim _{\sigma_{p P} \rightarrow 0}\left(\phi_{1 a}+\phi_{2}\right)=\delta_{1} \frac{L_{1}-L_{2}-v_{2}\left(T_{1}-T_{2}\right)}{v_{1}-v_{2}} .
$$

The substitution of the classical relations $L_{1,2}=v_{1,2} T_{1,2}$ in the above equation shows indeed that the phase difference depends only on the classical propagation time of the neutrino:

$$
\lim _{\sigma_{p P} \rightarrow 0}\left(\phi_{1 a}+\phi_{2}\right)=\delta_{1} T_{1}=\frac{\delta m_{1 a}^{2} L_{1}}{2 p_{D_{1}}} .
$$

In short the probability to detect both muon and neutrino can be interpreted as the result of a neutrino oscillation alone. Recall that the above relation can be obtained in a simple way in one dimension by energy-momentum conservation at the source.

As regards the experimental data, oscillations of recoil particles have not been probed (in the case of the $\pi \rightarrow \mu \nu$, it would only be possible for a neutrino oscillation length shorter than the muon decay length). However the neutral kaon mass difference obtained from strangeness oscillation experiments is consistent with the one obtained from regeneration experiments [201].

\subsection{Oscillations of correlated mesons}

We compute here the amplitude associated to the correlated oscillations of a pair of neutral mesons produced in $e^{+} e^{-}$annihilation. The phenomenology of correlated $K$ and $B$ mesons is discussed in 108, 79, 159, 77] and in [82, 83, 62, 115, 77, respectively.

Each meson oscillates between its components $P_{L}-P_{H}$, before decaying into final states $f_{1}\left(k_{1}\right)$ and $f_{2}\left(k_{2}\right)$ at spacetime points $y_{1}$ and $y_{2}$ :

$$
e^{+} e^{-} \rightarrow \mathcal{R}(q) \rightarrow P^{0} \bar{P}^{0} \rightarrow f_{1}\left(k_{1}\right) f_{2}\left(k_{2}\right)
$$

where $q, k_{1}$ and $k_{2}$ are the corresponding energy-momenta.

Since each final state can be produced by either $P^{0}$ or $\bar{P}^{0}$, the two amplitudes arising from the exchange of $P^{0}$ and $\bar{P}^{0}$ as intermediate states must be coherently added. The resonance 
quantum numbers of $\phi$ and $\Upsilon(4 \mathrm{~s}), J^{P C}=1^{--}$, are conserved by the strong interactions at the source, so that the pair $P^{0} \bar{P}^{0}$ is in an antisymmetric state under $P$ and under $C$. Thus the relative sign of the two contributions to $\mathcal{R} \rightarrow f_{1} f_{2}$ is negative [196].

The total amplitude before antisymmetrization is given by Eq. (200). In section 10.2, it was shown that the energy-momentum correlation at the source can be neglected for the resonances $\phi$ and $\Upsilon(4 \mathrm{~s})$, so that the overlap function factorizes as in Eq. (201). The amplitude for correlated oscillations can thus be approximated by the product of two amplitudes, each corresponding to a single oscillation. Furthermore, it was shown in section 8.3 that the oscillation amplitude, in the case of nearly degenerate unstable particles, can be replaced by the effective amplitude (189) depending only on the distance $L$. In the end the antisymmetrized amplitude to detect a $f_{1}$ at distance $L_{1}$ and a $f_{2}$ at distance $L_{2}$ can be written as

$$
\begin{aligned}
& \mathcal{T}_{f_{1} f_{2}} \sim \mathcal{M}_{P}\left(\left(\mathcal{M}_{01} \mathcal{M}_{\overline{0} 1}\right) V^{-1} e^{-i M \frac{m_{0} L_{1}}{p_{1}}} V\left(\begin{array}{l}
1 \\
0
\end{array}\right)\right)\left(\left(\begin{array}{ll}
\mathcal{M}_{02} & \mathcal{M}_{\overline{0} 2}
\end{array}\right) V^{-1} e^{-i M \frac{m_{0} L_{2}}{p_{2}}} V\left(\begin{array}{l}
0 \\
1
\end{array}\right)\right) \\
& \text { - same expression with }\left(\begin{array}{l}
1 \\
0
\end{array}\right) \leftrightarrow\left(\begin{array}{l}
0 \\
1
\end{array}\right) \text {, }
\end{aligned}
$$

where $\mathcal{M}_{P}=\mathcal{M}\left(\mathcal{R} \rightarrow P^{0} \bar{P}^{0}\right)$. The matrix $M$ is defined by $M=\operatorname{diag}\left(m_{L}-i \Gamma_{L} / 2, m_{H}-i \Gamma_{H} / 2\right)$, $m_{0}$ is the mass of the kaon in the degenerate limit and $p_{j}$ are the norms of the 3-momenta of the final states $f_{j}$. The diagonalization matrix $V$ is given by Eq. (192). The amplitudes $\mathcal{M}_{0 j}$ and $\mathcal{M}_{\overline{0} j}$ stand for $\mathcal{M}\left(P^{0} \rightarrow f_{j}\right)$ and $\mathcal{M}\left(\bar{P}^{0} \rightarrow f_{j}\right)$, respectively.

The amplitude can be written as

$$
\begin{aligned}
\mathcal{T}_{f_{1} f_{2}} & \sim \mathcal{M}_{P} \mathcal{M}_{01} \mathcal{M}_{02} \frac{\left(1+\sigma \mu_{f_{1}}\right)\left(1+\sigma \mu_{f_{2}}\right)}{2 \sigma} \\
& \times\left(-\eta_{f_{2}} e^{-i\left(m_{L}-\frac{i}{2} \Gamma_{L}\right) \frac{m_{0} L_{1}}{p_{1}}-i\left(m_{H}-\frac{i}{2} \Gamma_{H}\right) \frac{m_{0} L_{2}}{p_{2}}}+\eta_{f_{1}} e^{-i\left(m_{H}-\frac{i}{2} \Gamma_{H}\right) \frac{m_{0} L_{1}}{p_{1}}-i\left(m_{L}-\frac{i}{2} \Gamma_{L}\right) \frac{m_{0} L_{2}}{p_{2}}}\right),
\end{aligned}
$$

where $\mu_{f}$ is defined by Eq. (194) and $\eta_{f}=\left(1-\sigma \mu_{f}\right) /\left(1+\sigma \mu_{f}\right)$. Note that the amplitude vanishes for identical final states $\left(f_{1}=f_{2}\right.$ and $\left.p_{1}=p_{2}\right)$ and identical propagation distances $\left(L_{1}=L_{2}\right)$, as expected [100]. In the center-of-mass frame, the equality of momenta $p_{1}=p_{2} \equiv p$ implies that the interference term oscillates like

$$
\cos \left(\frac{m_{0}\left(m_{H}-m_{L}\right)}{p}\left(L_{1}-L_{2}\right)\right) .
$$

This oscillation formula coincides with the quantum-mechanical result obtained with the equal time prescription, whereas the different time prescription leads to an oscillation length shorter by a factor 2 [245]. As already explained in section 2.3, the quantum field treatment shows that neither prescription is meaningful, since the interference takes place over a space and time range, determined by the effective 'wave packet' width. We can only say that, in the end, the different mass eigenstates have the same average propagation time or distance, so that the oscillation formula will agree with the one obtained with the identical time prescription. As regards the experimental data, Kayser [178] has shown, in the case of the process $\Upsilon \rightarrow B^{0} \overline{B^{0}}$, that the mass difference obtained from the oscillation formula (210) is in agreement with the mass difference extracted from single $B$ oscillations. 


\section{Summary and outlook}

Although the plane wave derivation of the vacuum oscillation formula has often been criticized in the literature, it is still used in most articles and textbooks. There are two reasons for this. First, the oscillation formula obtained in this way is believed to be correct by most physicists, in spite of the numerous inconsistencies present in its derivation. Second, the other approaches are not felt to be completely satisfying, with the consequence that more sophisticated treatments are not considered worth the effort.

Let us first point out that we do not claim that the oscillation formula obtained with the plane wave approach should be revised. However we have argued that this approach becomes unacceptable when all its inconsistencies are added up: the perfect knowledge of the momentum precludes spatial oscillations, observability conditions (such as $\sigma_{x} \lesssim L^{\text {osc }}$ or $L \lesssim L^{\text {coh }}$ ) are not taken into account, flavor states are ill-defined, unstable oscillating particles cannot be consistently described in that model. Furthermore, the plane wave derivation requires two prescriptions: the classical propagation condition $(|v t-x| \ll t)$ and the equal time prescription $\left(t_{i}=t_{j}\right)$. Whereas the first prescription can be justified in a quantum-mechanical wave packet approach, the second prescription can only be proved right in a field-theoretical treatment. This is an important argument in favor of the latter approach, since the different time prescription $\left(t_{i} \neq t_{j}\right)$ leads to oscillation formulas differing significantly from the standard result. We have also shown that an energy-momentum prescription ( $\operatorname{such}$ as $E_{i}=E_{j}$ or $p_{i}=p_{j}$ ) is not necessary to derive the oscillation formula.

Once the necessity of a more sophisticated approach is understood, we are faced with the choice between two main methods: on the one hand, a quantum-mechanical treatment associating wave packets with the propagating mass eigenstates and, on the other hand, a fieldtheoretical treatment where the oscillating particle is considered as an internal line of a Feynman diagram. The first possibility is rich in physical insights (such as the existence of a coherence length) but not satisfactory in many respects: flavor states are still ill-defined, the equal time prescription is still needed, the nonrelativistic limit is problematic if the mass eigenstates are not nearly degenerate, the size of the wave packet is hard to estimate, the coherence length is not well-defined in the nonrelativistic limit, the case of a vanishing energy uncertainty is not included, and finally the treatment is inadequate for unstable particles. Whereas most of these problems are solved in the interacting wave packet model of Giunti [138], this last model requires quantum field theory to compute the interactions and is as complex as the external wave packet model.

Derivations of the oscillation formula resorting to field-theoretical methods are not very popular, although they seem the only way out. The first reason is that they are thought to be very complicated. The second reason is that the existing quantum field computations of the oscillation formula do not agree in all respects. The aim of our report was to counter both

objections. The first of these is easily refuted by noting that the oscillation formula for a stable particle can be derived in a very simple field-theoretical model, the Kobzarev et al. model [187], 
in which the source and the detector are approximated by infinitely heavy nuclei. This is the simplest model of all in which the oscillation formula can be consistently derived. The second objection could only be countered by a detailed and complete computation of the oscillation formula with all approximations carefully considered.

In this report, we have shown that all existing field-theoretical treatments (with the noteworthy exception of the Blasone-Vitiello approach [72]) can be included in the so-called external wave packet model, where the oscillating particle is described as an internal line of a Feynman diagram and propagates between a source and a detector localized with in- and outgoing wave packets. In particular, we have paid attention to the case of a vanishing energy uncertainty (stationary limit) and its wave packet interpretation, so that we could prove that the GrimusStockinger model [150] is a subcase of the external wave packet model. Two other limits have been considered. First, we have proved that oscillations near the source can be described by the standard oscillation formula, contrary to what was claimed by Ioannisian and Pilaftsis 163. Second, we have also shown that oscillations far from a well-localized source are also described by the standard oscillation formula, contrary to Shtanov's claim [242]. In order to include all these cases in our formalism, it has been necessary to evaluate the amplitude with two approximation schemes: Laplace's and stationary phase methods. As a result, the propagation range was divided into three regimes, distinguished by the dispersion (or spreading in space-time) of the amplitude. In the first regime, near the source, the dispersion is negligible and Laplace's method is sufficient. This case corresponds to Ioannisian and Pilaftsis' limit. In the second regime, the transversal dispersion becomes important, so that both approximation methods need to be used. In the third regime, far from the source, the longitudinal dispersion becomes important and the stationary phase method is sufficient. This case corresponds to Shtanov's limit. We have also discussed another method of computation (in energy-momentum space) valid for stable oscillating particles, as it clears up in which sense interference occurs between same energy states.

The computation of the intrinsic decoherence at the wave packet level has yielded two wellknown observability conditions for oscillations. However, our physical explanation differs from the standard one by taking into account the dispersion of the amplitude, so that the origin of decoherence depends on the distance at which it occurs. The first condition of observability of oscillations, $L_{i j}^{o s c} \gg \sigma_{x}$, is either due to the initial spread of the associated 'wave packets', or to their constant overlap at large distance. Similarly the existence of a coherence length (yielding the second observability condition $L \ll L_{i j}^{c o h}$ ) is either due to the separation of 'wave packets', or to their dispersion. Our 3-dimensional treatment has also yielded a third observability condition, $\left|\mathbf{p}_{0} \times \mathbf{l}\right| \lesssim \sigma_{p}$ (modified into $\left|\mathbf{v}_{0} \times \mathbf{L}\right| \lesssim v_{0} \sigma_{x}$ at short distance) which has the obvious geometrical interpretation of constraining the propagation within a cone (respectively a cylinder at short distance). It is now clear that the coherence length tends to infinity in the stationary limit, as noted by Kiers, Nussinov and Weiss [182].

The oscillation of unstable particles, whether mesons or neutrinos, has been analyzed in the same framework, with the result that the oscillation formula is only modified, as expected, by 
the well-known exponential decay term. Nonexponential corrections to the oscillation formula have been estimated for the first time in quantum field theory. Finally we have given a fieldtheoretical treatment of correlated oscillations, with the aim of disproving the existence of recoil oscillations.

Our work confirms that the standard vacuum oscillation formula given by Eqs. (12) and (14) is correct if the observability conditions mentioned above are satisfied. This fact is rather remarkable, in the light of the numerous inconsistencies which were present in the plane wave derivation of this formula. It can be explained by the following observations:

- the energy and momentum present in the phase are linked by the on-shell condition, and the average propagation time and length are linked by a constraint equivalent to a wave packet localization in space-time. For these reasons, the $E_{j} T$ and $p_{j} L$ components of the phase cancel but for the usual oscillating factor $\frac{\delta m^{2} L}{2 p}$;

- only same energy components interfere because of the time average, so that the timeaveraged transition probability computed in the external wave packet model is equivalent to the energy-integrated transition probability computed with plane waves;

- from the previous argument, decoherence effects arising from external wave packets cannot be distinguished from decoherence effects arising from averages over the production (and detection) region and over the energy spectrum; since the former effects are dominated by the latter, they have no influence on the oscillation formula.

For ten years, new experimental results in neutrino physics have stimulated the research on the theory of particle oscillations, leading to the development of numerous (and sometimes conflicting) field-theoretical approaches. In this report, we have tried to unify these treatments and to extend them to oscillations of $K$ and $B$ mesons. We indeed believe that the neutrino oscillation formula is much strengthened by considering it in the same framework as the mesonic oscillation formula, whose parameters have been confirmed by other methods such as regeneration in matter. We hope to have convinced the reader that field-theoretical models provide, on the one hand, the most secure foundation to the oscillation formula and give, on the other hand, a good physical understanding of oscillation phenomena, whatever the particles involved.

One case needing further investigation is the decay in flight of the source of the oscillating particle, which is important for atmospheric neutrinos and some laboratory neutrino experiments. It would also be interesting to apply a field-theoretical treatment to cascade decays, such as $B \rightarrow J / \psi K \rightarrow J / \psi f_{K}$, where double-flavor oscillations occur. These processes are useful for the determination of the signs of the $B_{H} B_{L}$ mass and lifetime differences [32, 179, 77, 233].

The field-theoretical approach faces its biggest challenge in the description of neutrino oscillations in matter. A first task consists in the rederivation of the MSW oscillation formula (see [205, 214, 223, 87] for a few attempts). Another difficult task is to describe the nonstationary evolution of neutrinos in supernovae or in the early universe, where interaction rates are in competition with the flavor oscillation period 234, 86]. 
The turn of the century marks a new golden age for CP violation experiments, thanks to the $B$ factories, as well as the golden era of neutrino oscillation experiments. These favorable auspices should be a great stimulation for further research on the theoretical foundations of oscillations.

\section{Acknowledgments}

I am deeply indebted to Jeanne De Jaegher for a thorough reading of the manuscript, which led to lengthy discussions on the quantum mechanics of oscillations, as well as the rewriting of some sections. Discussions with Jean Pestieau, Andreas Veithen, Jacques Weyers, Carlo Giunti and Christian Cardall are gratefully acknowledged. I thank Giuseppe Vitiello and Massimo Blasone for having organized in Vietri a workshop dedicated to the theoretical foundations of particle oscillations. It is a pleasure to thank Michel and Brita Beuthe for their comments as well as for travel support. I must also express my gratitude to Karel and Cécile De Jaegher and to Attilio Rivoldini for their computer-related assistance. Finally, I thank the Université Catholique de Louvain and its Institut de Physique Théorique for financial support.

\section{Appendix: the Jacob-Sachs theorem}

Let $\psi(E, \mathbf{p})$ be a function which is distinct from zero only within certain bounds:

$$
\psi(E, \mathbf{p}) \neq 0 \text { for } 0<M_{1}^{2}<p^{2}<M_{2}^{2} \text { and } E \geq 0
$$

with $p^{2}=E^{2}-\mathbf{p}^{2}$. On this interval $\psi(E, \mathbf{p})$ is taken to be infinitely differentiable. Let the function $I(T)$ be defined by

$$
I(T)=\int d E \psi(E, \mathbf{p}) G\left(p^{2}\right) e^{-i E T},
$$

where the function $G\left(p^{2}\right)$ is the complete scalar propagator in momentum space.

We are going to prove that the function $I(T)$ has the following asymptotic behavior:

$$
I(T) \stackrel{T \rightarrow \infty}{\longrightarrow} \frac{\pi Z}{\sqrt{z_{0}+\mathbf{p}^{2}}} \psi\left(\sqrt{z_{0}+\mathbf{p}^{2}}, \mathbf{p}\right) e^{-i \sqrt{z_{0}+\mathbf{p}^{2}}},
$$

where $z_{0}$ is the pole of the integrand and $Z$ is the residue. This section follows [166].

It will be easier to work with an overlap function depending explicitly on $p^{2}$, so we write $\psi\left(p^{2}, \mathbf{p}\right)$. We choose to implement the assumption of compact domain of $\psi\left(p^{2}, \mathbf{p}\right)$ by

$$
\begin{aligned}
& \psi\left(p^{2}, \mathbf{p}\right)=\left(p^{2}-M_{1}^{2}\right)^{n}\left(p^{2}-M_{2}^{2}\right)^{n} \Omega\left(p^{2}, \mathbf{p}\right) \text { for } 0<M_{1}^{2}<p^{2}<M_{2}^{2} \quad(n>0), \\
& \psi\left(p^{2}, \mathbf{p}\right)=0 \text { otherwise. }
\end{aligned}
$$

The symmetrical behavior at $M_{1}^{2}$ and $M_{2}^{2}$ is chosen only to simplify the algebra. The function $\Omega$ is taken to be analytic over the domain of interest. 
Under the change of variable $z=p^{2}$, the function $I(T)$ becomes

$$
I(T)=\frac{1}{2} \int_{M_{1}^{2}}^{M_{2}^{2}} d z\left(z+\mathbf{p}^{2}\right)^{-\frac{1}{2}} \psi(z, \mathbf{p}) G(z) e^{-i \sqrt{z+\mathbf{p}^{2}} T} .
$$

All functions in the integrand are analytically continued in the complex plane. For example, the real propagator is considered as the limit of an analytic function of the complex variable $z$ :

$$
G\left(p^{2}\right)=\lim _{z \rightarrow p^{2}+i \epsilon} G(z) .
$$

The full propagator associated to a scalar field can be written in the Schwinger's spectral representation [241] as

$$
G(z)=\frac{i}{z-M_{0}^{2}-\Pi(z)},
$$

where $M_{0}$ is the bare mass of the scalar field and $\Pi(z)$ is an analytic function defined by

$$
\Pi(z)=z \int_{b^{2}}^{\infty} d s \frac{\sigma(s)}{z-s}
$$

which can be interpreted as the self-energy of the scalar field. The positive real function $\sigma(x)$ satisfies $\sigma(x)=0$ for $x \leq b^{2} . b^{2}$ is the invariant mass of the lightest multi-particle state in interaction with the scalar field corresponding to the propagator. This spectral representation has the advantage of being in direct correspondence with the full propagator (Eq. (26)), obtained in perturbation theory by an infinite sum over the self-energy insertions. If $x$ is on the real axis, it can be checked that

$$
\Pi(x+i \epsilon)-\Pi(x-i \epsilon)=-2 \pi i x \sigma(x) .
$$

The self-energy can be written as

$$
\Pi(x \pm i \epsilon)=\delta M^{2}+u(x) \mp i v(x),
$$

where $v(x)=\pi x \sigma(x)$ and $\delta M^{2}$ is an infinite constant which is absorbed in the renormalized mass $M^{2}$ :

$$
M^{2}=M_{0}^{2}+\delta M^{2} .
$$

Note that $M$ is not the physical mass since it is not the pole of the propagator.

The propagator $G(z)$ has branch points at the thresholds corresponding to the multi-particle states. The first branch point is at $z=b^{2}$. We assume that the other branch points are above $M_{2}^{2}$. The analytic continuation of $G(z)$ into the second Riemann sheet 78 is such that the analytically continued function $G_{I I}(z)$ just below the real axis is equal to the original function just above the real axis. The analytically continued function $\Pi_{I I}(z)$ of the function $\Pi(z)$ is defined in the same way, $\Pi_{I I}(x-i \epsilon)=\Pi(x+i \epsilon)$, so that

$$
G_{I I}(z)=\frac{i}{z-M_{0}^{2}-\Pi_{I I}(z)} .
$$

$G_{I I}(z)$ has a pole at $z_{0}=m^{2}-i m \Gamma$.

Different cases have to be considered, according to the stability or instability of the particle, and according to the positions of the pole $z_{0}$ and threshold $b^{2}$. The pole is assumed to be inside the contour (otherwise the particle cannot be emitted). 


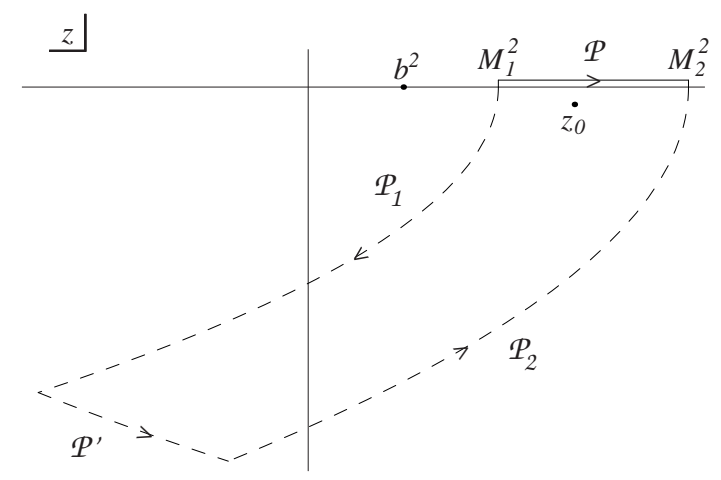

Figure 9: Integration contour if $b<M_{1}<m<M_{2}$.

Unstable particle, with $b<M_{1}<m<M_{2}$

The path of integration is shown on Fig. 9. The analytic expressions of the paths $\mathcal{P}_{j}$ read

$$
\mathcal{P}_{j}: z=\left(-i \omega+\sqrt{M_{j}^{2}+\mathbf{p}^{2}}\right)^{2}-\mathbf{p}^{2} \quad(j=1,2),
$$

where $\omega$ ranges from zero to $\omega_{\infty}$ on $\mathcal{P}_{1}$ and from $\omega_{\infty}$ to zero on $\mathcal{P}_{2}$. The analytic expression of the path $\mathcal{P}^{\prime}$ reads

$$
\mathcal{P}^{\prime}: z=\left(-i \omega_{\infty}+\sqrt{M^{2}+\mathbf{p}^{2}}\right)^{2}-\mathbf{p}^{2},
$$

where $M$ ranges from $M_{1}$ to $M_{2}$, with the limit $\omega_{\infty} \rightarrow \infty$ understood.

The integral $I(T)$ is equal to

$$
I(T)=J+J_{1}+J_{2}+J^{\prime}
$$

$J$ is the contribution of the pole $z_{0}$, whereas $J_{1}, J_{2}$ and $J^{\prime}$ are the contributions of $\mathcal{P}_{1}, \mathcal{P}_{2}$ and $\mathcal{P}^{\prime}$, respectively. Their analytic expressions read

1. Contribution of the pole:

$$
J=Z \pi\left(z_{0}+\mathbf{p}^{2}\right)^{-\frac{1}{2}} \psi\left(z_{0}, \mathbf{p}\right) e^{-i \sqrt{z_{0}+\mathbf{p}^{2}} T} .
$$

Setting $\Delta M \cong\left|m-M_{1,2}\right|$, a rough estimate of $J$ is given by $J \sim m^{2 n-1}(\Delta M)^{2 n} e^{-\Gamma T / 2}$.

2. Contribution of the sickle-shaped paths:

$$
J_{j}=i(-1)^{j} e^{-i \sqrt{M_{j}^{2}+\mathbf{p}^{2}} T} \int_{0}^{\infty} d \omega \psi(z(\omega), \mathbf{p}) G_{I I}(z(\omega)) e^{-\omega T} .
$$

At large $T$, the dominant contribution to the integral comes from the $\omega$ values near zero, because of the decreasing exponential. Since the integrand tends to zero with $\omega$ $\left.\left(\psi\left(M_{j}^{2}, \mathbf{p}\right)=0\right)\right)$, the asymptotic behavior of the integral depends on the way the integrand tends to zero. Setting $y=\omega T$ and expanding in $1 / T$, we obtain:

$$
J_{j} \sim\left(M_{2}^{2}-M_{1}^{2}\right)^{n}\left(M_{j}^{2}+\mathbf{p}^{2}\right)^{n / 2} \Omega\left(M_{j}^{2}, \mathbf{p}\right) G_{I I}\left(M_{j}^{2}\right) T^{-(n+1)} e^{-i \sqrt{M_{j}^{2}+\mathbf{p}^{2}} T} .
$$


The corrections to this formula are of order $(\Delta M T)^{-(n+2)}$, where $\Delta M \approx M_{j}-m$, that is, they are of the order of the uncertainty on the particle mass. It is assumed that the function $\Omega(z, \mathbf{p})$ diverges more slowly than $\exp (-\omega T)$ on the paths $\mathcal{P}_{j}$ as $\omega$ tends to infinity. This assumption is true for a wide class of functions, in particular for Gaussians, whereas their path integral diverges on half-circles at infinity. A rough estimate of $J_{j}$ is given by $J_{j} \sim m^{2 n-1}(\Delta M)^{n-1} T^{-(n+1)}$.

3. Contribution from the path at infinity:

$$
J^{\prime}=e^{-\omega_{\infty} T} \int_{M_{1}^{2}}^{M_{2}^{2}} d M \frac{M}{\sqrt{M^{2}+\mathbf{p}^{2}}} \psi(z(M)) G_{I I}(z(M)) e^{-i \sqrt{M^{2}+\mathbf{p}^{2}} T} .
$$

If $\Omega$ satisfies the same conditions at infinity as above, $J^{\prime} \sim \exp \left(-\omega_{\infty} T\right)$ and tends to zero as $\omega_{\infty} \rightarrow \infty$.

In conclusion, the contribution of the pole is a decreasing exponential in $T$, whereas the contributions due to the bounded character of the energy spectrum decrease in inverse powers of $T$.

At small $T, J_{j} / J \sim(\Delta M T)^{-(n+1)}$, which is nonnegligible for $\Delta M T \lesssim 1$. Below that value, the asymptotic evaluation of $J_{j}$ is not valid anymore, because terms in $(\Delta M T)^{-(n+2)}$ have been neglected.

First, let us consider weakly decaying particles. In the case of the $K_{S}^{0}$, the mass is measured with a precision of $\Delta M \approx 10^{-2} \mathrm{MeV}$. Thus nonexponential corrections will be important for $T \lesssim 10^{-19} \mathrm{~s}$, which is not observable since the $K_{S}^{0}$ lifetime is $0.89 \times 10^{-10} \mathrm{~s}$. In the case of the $B^{0}, \Delta M \approx 2 \mathrm{MeV}$, so that nonexponential corrections will be important for $T \lesssim 10^{-22} \mathrm{~s}$, which is not observable since the $B^{0}$ lifetime is $1.29 \times 10^{-12} \mathrm{~s}$. Next, let us next consider resonances. In the example of $\Delta(1232), \Delta M \approx 2 \mathrm{MeV}$, so that nonexponential corrections are important for $T \lesssim 10^{-22} \mathrm{~s}$, which is large compared to the inverse width equal to $5 \times 10^{-24} \mathrm{~s}$. Thus the propagation of resonances can never be modelized by the contribution of the pole alone.

In the case of the weakly decaying particles, power law corrections are also important at large times and dominate if

$$
\Gamma T \gtrsim 2(n+1) \ln (\Delta M T)=2(n+1)(\ln (\Gamma T)+\ln (\Delta M / \Gamma))
$$

For $K_{S}^{0}$, the two-particle threshold is characterized by $n=1 / 2$ and $\Delta M / \Gamma \sim \mathcal{O}\left(10^{10}\right)$, so that the threshold of the nonexponential behavior is given by $\Gamma T \gtrsim 69$, at which time the amplitude will be much too small to be observable. For $B^{0}$, taking $n=1 / 2$ and $\Delta M / \Gamma \sim \mathcal{O}\left(10^{9}\right)$, we obtain $\Gamma T \gtrsim 62$, with the same conclusion as above.

To sum up the analysis of the propagation of a weakly decaying particle (with no multiparticle thresholds included in the spectrum), the function $I(T)$ is very well approximated in the observable time domain by Eq. (211). 


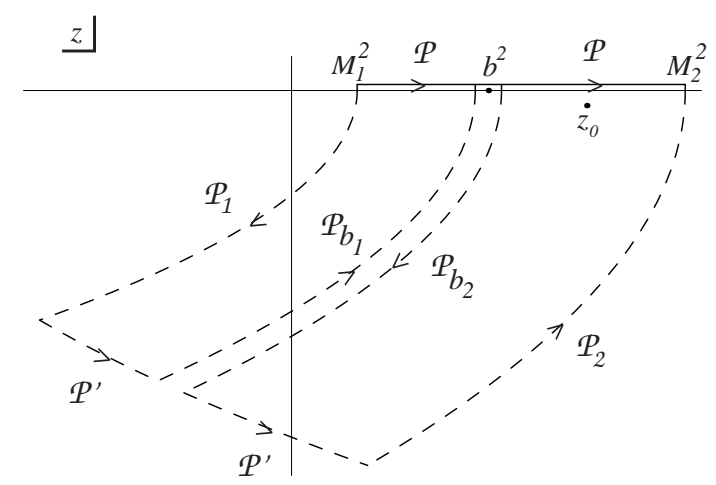

Figure 10: Integration contour if $M_{1}<b<m<M_{2}$.

\section{Unstable particle, with $M_{1}<b<m<M_{2}$}

In this case, the multi-particle threshold is included in the energy spectrum. It generates new power law corrections to the amplitude.

The path of integration is shown on Fig. 10. The contributions of the paths $\mathcal{P}_{j}$ are the same as in the previous case, except that $G(z)$ replaces $G_{I I}(z)$ on $\mathcal{P}_{1}$, since this path lies on the first Riemann sheet. The asymptotic value of $J_{j}$ as $T \rightarrow \infty$ does not change. The contributions of the paths $\mathcal{P}_{b_{j}}$ do not cancel each other out, since they do not lie on the same Riemann sheet. Their sum reads

$$
\begin{aligned}
J_{b} & =\frac{1}{2} \int_{\mathcal{P}_{b}} d z\left(z+\mathbf{p}^{2}\right)^{-\frac{1}{2}} \psi(z, \mathbf{p})\left(G_{I I}(z)-G(z)\right) e^{-i \sqrt{z+\mathbf{p}^{2}} T} \\
& =-i e^{-i \sqrt{b^{2}+\mathbf{p}^{2}} T} \int_{0}^{\infty} d \omega \psi(z(\omega), \mathbf{p})\left(G_{I I}(z)-G(z)\right) e^{-\omega T} .
\end{aligned}
$$

The analytic expression of the paths $\mathcal{P}_{b}$ reads

$$
\mathcal{P}_{b}: z=\left(-i \omega+\sqrt{b^{2}+\mathbf{p}^{2}}\right)^{2}-\mathbf{p}^{2},
$$

where $\omega$ ranges from zero to $\omega_{\infty}$, with the limit $\omega_{\infty} \rightarrow \infty$ understood. The function $I(T)$ is equal to

$$
I(T)=J+J_{1}+J_{2}+J_{b}+J^{\prime} .
$$

The asymptotic behavior of $J_{b}$ is studied in the same way as for $J_{j}$. As before, the dominant contribution to $J_{b}$, for large $T$, comes from $\omega$ values near zero. Again, we set $y=\omega T$ and expand the integrand in $1 / T$. Recall that $G_{I I}(z)$ is defined by the analytic continuation of $G(x+i \epsilon)$ below the cut, $G_{I I}(x-i \epsilon)=G(x+i \epsilon)$. Its discontinuity through the cut can be computed with Eqs. 212), (214) and (215):

$$
G_{I I}(x-i \epsilon)-G(x-i \epsilon)=\frac{2 v(x)}{\left(x-M^{2}-u(x)\right)^{2}+v^{2}(x)} .
$$


In the example of $K^{0}$, the self-energy is given at first order in $g^{2}$ by the pion bubble diagram (where $g$ is the coupling constant between a kaon and two pions), yielding

$$
v(z)=\frac{g^{2}}{4 \pi} \sqrt{1-b^{2} / z}
$$

where $b=2 m_{\pi}$ is the two-pion threshold. The function $u(z)$ is also of order $g^{2}$.

The evaluation of $J_{b}$ with the same asymptotic method as used for $J_{j}$ gives

$$
J_{b}=-i(-2 i \pi)^{\frac{1}{2}} T^{-3 / 2} \frac{g^{2}}{4 \pi b} \frac{\left(b^{2}+\mathbf{p}^{2}\right)^{1 / 4}}{\left(b^{2}-M^{2}-u\left(b^{2}\right)\right)^{2}} \psi\left(b^{2}, \mathbf{p}\right) e^{-i \sqrt{b^{2}+\mathbf{p}^{2}} T} .
$$

The corrections to this formula are of order $(Q T)^{-5 / 2}$, where $Q \equiv M-b$ is the energy release on decay of the unstable particle. Noting that cutting rules give $v\left(m^{2}\right)=m \Gamma$, the coupling constant $g^{2}$ can be replaced by its expression in function of $\Gamma, m$ and $Q$. A rough estimate of $J_{b}$ is then given by

$$
J_{b} \sim(Q T)^{-3 / 2} \frac{\Gamma}{Q} \frac{1}{m} \psi\left(b^{2}, \mathbf{p}\right) .
$$

At small $T$, the ratio $J_{b} / J$ is of the order of

$$
\frac{J_{b}}{J} \sim(Q T)^{-3 / 2} \frac{\Gamma}{Q}
$$

which is much smaller than 1 if $Q T \gg 1$ and $\Gamma / Q \ll 1$. This is the case for weakly decaying particles. For example, $\Gamma / Q \approx 10^{-14}$ for $K_{S}^{0}$. It is not true for resonances. For example, $\Gamma / Q \approx 0.8$ for $\Delta(1232)$. Below the value $Q T \approx 1$, the asymptotic computation of $J_{b}$ is not valid anymore since corrections in $(Q T)^{-5 / 2}$ have been neglected.

At large $T$, the power law contribution $J_{b}$ dominates the pole contribution if

$$
\Gamma T-3 \ln (\Gamma T) \gtrsim 5 \ln (Q / \Gamma)
$$

so that the nonexponential time thresholds are given by $\Gamma_{S} T \gtrsim 165$ for $K_{S}^{0}, \Gamma_{L} T \gtrsim 202$ for $K_{L}^{0}$ and $\Gamma_{L, H} T \gtrsim 157$ for $B_{L, H}^{0}$ (in the last case, we have taken $Q=1 \mathrm{MeV}$ ). Thus nonexponential effects are not observable at large times for weakly decaying particles. In the case of resonances, the contribution of $J_{b}$ is always of the same order or larger than the pole contribution. Resonances do not propagate macroscopically, since their width is of the same order as the typical energy of the processes.

To sum up the analysis of the propagation of a weakly decaying particle, including the multi-particle thresholds, the function $I(T)$ is very well approximated in the observable time domain by the Eq. (211).

\section{Stable particle, with $M_{1}<m<M_{2}<b$}

This case can be examined like the first one, except that the pole is real, so that the result is given by Eq. (211), with $\Gamma=0$. There is no exponential decrease of the amplitude. Power law corrections are negligible at large $T$. 
Stable particle, with $M_{1}<m<b<M_{2}$

This case can be examined like the second one, except that the pole is real. The result is again given by Eq. (211), with $\Gamma=0$. The ratio $J_{b} / J$ is of the order of $J_{b} / J \sim(m T)^{-3 / 2} g^{2} / Q^{2}$, where $g$ is the coupling constant with the particles produced at the threshold. The contribution of $J_{b}$ is too small to be observed at small $T$ in the current experiments, and is negligible at large $T$.

\section{References}

[1] A. I. Abazov et al., Search for neutrinos from Sun using the reaction ${ }^{71} G a\left(\nu_{e}, e^{-}\right){ }^{71} G e$, Phys. Rev. Lett. 67 (1991) 3332.

[2] J. N. Abdurashitov et al. [SAGE Collaboration], Measurement of the solar neutrino capture rate by SAGE and implications for neutrino oscillations in vacuum, Phys. Rev. Lett. 83 (1999) 4686 arXiv:astro$\mathrm{ph} / 9907131$.

[3] K. Abe et al. [Belle Collaboration], Observation of large CP violation in the neutral B meson system, Phys. Rev. Lett. 87 (2001) 091802 arXiv:hep-ex/0107061.

[4] K. Abe et al. [Belle Collaboration], An improved measurement of mixing-induced CP violation in the neutral B meson system, arXiv:hep-ex/0208025.

[5] A. Acker, A. Joshipura and S. Pakvasa, A neutrino decay model, solar anti-neutrinos and atmospheric neutrinos, Phys. Lett. B 285 (1992) 371.

[6] A. Acker and S. Pakvasa, Solar neutrino decay, Phys. Lett. B 320 (1994) 320 arXiv:hep-ph/9310207.

[7] M. Adinolfi et al. [KLOE Collaboration], First results from $\phi \rightarrow K_{L} K_{S}$ decays with the KLOE detector, arXiv:hep-ex/0006035.

[8] D. V. Ahluwalia and T. Goldman, Interplay of non-relativistic and relativistic effects in neutrino oscillations, Phys. Rev. D 56 (1997) 1698 arXiv:hep-ph/9702308.

[9] Q. R. Ahmad et al. [SNO Collaboration], Measurement of the charged current interactions produced by ${ }^{8} B$ solar neutrinos at the Sudbury Neutrino Observatory, Phys. Rev. Lett. 87 (2001) 071301 arXiv:nuclex/0106015.

[10] Q. R. Ahmad et al. [SNO Collaboration], Direct evidence for neutrino flavor transformation from neutralcurrent interactions in the Sudbury Neutrino Observatory, Phys. Rev. Lett. 89 (2002) 011301 arXiv:nuclex/0204008.

[11] S. H. Ahn et al. [K2K Collaboration], Detection of accelerator produced neutrinos at a distance of 250-km, Phys. Lett. B 511 (2001) 178 arXiv:hep-ex/0103001].

[12] E. K. Akhmedov and J. Pulido, SNO and the neutrino magnetic moment solution of the solar neutrino problem, Phys. Lett. B 485 (2000) 178 arXiv:hep-ph/0005173.

[13] H. Albrecht et al. [ARGUS Collaboration], Observation of $B^{0} \bar{B}^{0}$ Mixing, Phys. Lett. B 192 (1987) 245.

[14] E. Alfinito, M. Blasone, A. Iorio and G. Vitiello, Squeezed neutrino oscillations in quantum field theory, Phys. Lett. B 362 (1995) 91 arXiv:hep-ph/9510213.

[15] W. W. Allison et al. [Soudan-2 Collaboration], The atmospheric neutrino flavor ratio from a 3.9 fiducial kiloton-year exposure of Soudan 2, Phys. Lett. B 449 (1999) 137 arXiv:hep-ex/9901024.

[16] M. Altmann et al. [GNO Collaboration], GNO solar neutrino observations: results for GNO I, Phys. Lett. B 490 (2000) 16 arXiv:hep-ex/0006034.

[17] L. Alvarez-Gaumé, C. Kounnas, S. Lola and P. Pavlopoulos, Violation of time-reversal invariance and CPLEAR measurements, Phys. Lett. B 458 (1999) 347 [arXiv:hep-ph/9812326].

[18] M. Ambrosio et al. [MACRO Collaboration], Measurement of the atmospheric neutrino-induced upgoing muon flux using MACRO, Phys. Lett. B 434 (1998) 451 arXiv:hep-ex/9807005.

[19] H. Anada and H. Nishimura, Neutrino vacuum oscillation and neutrino burst from SN1987 A, Phys. Rev. D 37 (1988) 552.

[20] H. Anada and H. Nishimura, Coherence condition for resonant neutrino oscillations, Phys. Rev. D 41 (1990) 2379. 
[21] B. Ancochea, A. Bramon, R. Muñoz-Tapia and M. Nowakowski, Space-dependent probabilities for $K^{0} \bar{K}^{0}$ oscillations, Phys. Lett. B 389 (1996) 149 arXiv:hep-ph/9605454.

[22] B. Ancochea, A. Bramon and M. Nowakowski, Bell-inequalities for $K^{0} \bar{K}^{0}$ pairs from $\phi$-resonance decays, Phys. Rev. D 60 (1999) 094008 arXiv:hep-ph/9811404.

[23] V. B. Anikeev et al., Use of the big liquid argon spectrometer BARS for neutrino and cosmic-ray studies, Nucl. Instrum. Meth. A 419 (1998) 596.

[24] P. Anselmann et al. [GALLEX Collaboration], Solar neutrinos observed by Gallex at Gran Sasso, Phys. Lett. B 285 (1992) 376.

[25] A. Apostolakis et al. [CPLEAR Collaboration], An EPR experiment testing the non-separability of the $K^{0} \bar{K}^{0}$ wave function, Phys. Lett. B 422 (1998) 339.

[26] B. Armbruster et al. [KARMEN Collaboration], Anomaly in the time distribution of neutrinos from a pulsed beam stop source, Phys. Lett. B 348 (1995) 19.

[27] B. Armbruster et al., Upper limits for neutrino oscillations $\bar{\nu}_{\mu} \rightarrow \bar{\nu}_{e}$ from muon decay at rest, Phys. Rev. D 65 (2002) 112001 arXiv:hep-ex/0203021.

[28] C. Athanassopoulos et al. [LSND Collaboration], Evidence for $\bar{\nu}_{\mu} \rightarrow \bar{\nu}_{e}$ oscillation from the LSND experiment at the Los Alamos Meson Physics Facility, Phys. Rev. Lett. 77 (1996) 3082 arXiv:nucl-ex/9605003.

[29] C. Athanassopoulos et al. [LSND Collaboration], Evidence for $\nu_{\mu} \rightarrow \nu_{e}$ neutrino oscillations from LSND, Phys. Rev. Lett. 81 (1998) 1774 arXiv:nucl-ex/9709006.

[30] B. Aubert et al. [BABAR Collaboration], Observation of CP violation in the $B^{0}$ meson system, Phys. Rev. Lett. 87 (2001) 091801 arXiv:hep-ex/0107013.

[31] B. Aubert et al. [BABAR Collaboration], Measurement of the CP-violating asymmetry amplitude $\sin 2 \beta$, arXiv:hep-ex/0207042.

[32] Y. I. Azimov, $K^{0}$ decays as analyzers of $B^{0}$ decays: How to measure the sign of $\Delta m_{B}$, Phys. Rev. D 42 (1990) 3705.

[33] BABAR Collaboration (eds. P.F. Harrison and H.R. Quinn), The BABAR Physics Book: Physics at an Asymmetric B Factory, SLAC-R-0504 (1998) http://www.slac.stanford.edu/pubs/slacreports/slac-r504.htm].

[34] J. N. Bahcall, Neutrino Astrophysics (Cambridge University Press, Cambridge, 1989).

[35] J. N. Bahcall, ${ }^{7}$ Be solar neutrino line: A reflection of the central temperature distribution of the Sun, Phys. Rev. D 49 (1994) 3923.

[36] J. N. Bahcall, N. Cabibbo and A. Yahil, Are neutrinos stable particles?, Phys. Rev. Lett. 28 (1972) 316.

[37] J. N. Bahcall and S. C. Frautschi, Lepton non-conservation and solar neutrinos, Phys. Lett. 29B (1969) 623.

[38] J. N. Bahcall, M. C. Gonzalez-Garcia and C. Pena-Garay, Before and after: how has the SNO neutral current measurement changed things?, JHEP 0207 (2002) 054 arXiv:hep-ph/0204314.

[39] J. N. Bahcall, S. T. Petcov, S. Toshev and J. W. Valle, Tests of neutrino stability, Phys. Lett. B 181 (1986) 369.

[40] J. N. Bahcall, M. H. Pinsonneault and S. Basu, Solar models: Current epoch and time dependences, neutrinos, and helioseismological properties, Astrophys. J. 555 (2001) 990 arXiv:astro-ph/0010346.

[41] A. Bandyopadhyay, S. Choubey and S. Goswami, MSW mediated neutrino decay and the solar neutrino problem, Phys. Rev. D 63 (2001) 113019 arXiv:hep-ph/0101273.

[42] A. Bandyopadhyay, S. Choubey and S. Goswami, Neutrino decay confronts the SNO data, arXiv:hep$\mathrm{ph} / 0204173$.

[43] A. Bandyopadhyay, S. Choubey, S. Goswami and D. P. Roy, Implications of the first neutral current data from SNO for solar neutrino oscillation, Phys. Lett. B 540 (2002) 14 arXiv:hep-ph/0204286.

[44] J. Barranco, O. G. Miranda, T. I. Rashba, V. B. Semikoz and J. W. Valle, Confronting spin flavor solutions of the solar neutrino problem with current and future solar neutrino data, arXiv:hep-ph/0207326, and references therein.

[45] V. D. Barger, J. G. Learned, P. Lipari, M. Lusignoli, S. Pakvasa and T. J. Weiler, Neutrino decay and atmospheric neutrinos, Phys. Lett. B 462 (1999) 109 arXiv:hep-ph/9907421. 
[46] V. D. Barger, J. G. Learned, S. Pakvasa and T. J. Weiler, Neutrino decay as an explanation of atmospheric neutrino observations, Phys. Rev. Lett. 82 (1999) 2640 arXiv:astro-ph/9810121.

[47] V. Barger, D. Marfatia, K. Whisnant and B. P. Wood, Imprint of SNO neutral current data on the solar neutrino problem, Phys. Lett. B 537 (2002) 179 arXiv:hep-ph/0204253.

[48] V. Bargmann, On unitary ray representations of continuous groups, Ann. Math. 59 (1954) 1.

[49] L. Baulieu and R. Coquereaux, Photon-Z mixing in the Weinberg-Salam model, Ann. Phys. (N.Y.) 140 (1982) 163.

[50] J. F. Beacom and N. F. Bell, Do solar neutrinos decay?, Phys. Rev. D 65 (2002) 113009 arXiv:hep$\mathrm{ph} / 0204111$.

[51] R. Becker-Szendy et al., The electron- and muon-neutrino content of the atmospheric flux, Phys. Rev. D 46 (1992) 3720.

[52] F. Benatti and R. Floreanini, Massless neutrino oscillations, Phys. Rev. D 64 (2001) 085015 arXiv:hep$\mathrm{ph} / 0105303$, and references therein.

[53] C. M. Bender and S. A. Orszag, Advanced Mathematical Methods for Scientists and Engineers (McGrawHill, New York, 1978).

[54] Z. G. Berezhiani, G. Fiorentini, M. Moretti and A. Rossi, Fast neutrino decay and solar neutrino detectors, Z. Phys. C 54 (1992) 581.

[55] Z. G. Berezhiani, G. Fiorentini, A. Rossi and M. Moretti, Neutrino decay solution of the solar neutrino problem revisited, JETP Lett. 55 (1992) 151 [Pisma Zh. Eksp. Teor. Fiz. 55 (1992) 159].

[56] H. J. Bernstein and F. E. Low, The measurement of longitudinal coherence lengths in particle beams, Phys. Rev. Lett. 59 (1987) 951 [Erratum-ibid. 60 (1988) 755].

[57] R. A. Bertlmann and W. Grimus, How devious are deviations from quantum mechanics: the case of the $B^{0} \bar{B}^{0}$ system, Phys. Rev. D 58 (1998) 034014 arXiv:hep-ph/9710236.

[58] R. A. Bertlmann, W. Grimus and B. C. Hiesmayr, Quantum mechanics, Furry's hypothesis and a measure of decoherence in the $K^{0} \bar{K}^{0}$ system, Phys. Rev. D 60 (1999) 114032 arXiv:hep-ph/9902427.

[59] R. A. Bertlmann and B. C. Hiesmayr, Bell inequalities for entangled kaons and their unitary time evolution, Phys. Rev. A 63 (2001) 062112 arXiv:hep-ph/0101356, and references therein.

[60] M. Beuthe, Towards a unique formula for neutrino oscillations in vacuum, Phys. Rev. D 66 (2002) 013003 arXiv:hep-ph/0202068.

[61] M. Beuthe, G. López Castro and J. Pestieau, Field theory approach to $K^{0} \bar{K}^{0}$ and $B^{0} \bar{B}^{0}$ systems, Int. J. Mod. Phys. A 13 (1998) 3587 arXiv:hep-ph/9707369.

[62] I. I. Bigi and A. I. Sanda, CP violation in heavy flavor decays: predictions and search strategies, Nucl. Phys. B 281 (1987) 41.

[63] S. M. Bilenky and C. Giunti, Seesaw type mixing and $\nu_{\mu} \rightarrow \nu_{\tau}$ oscillations, Phys. Lett. B 300 (1993) 137 arXiv:hep-ph/9211269.

[64] S. M. Bilenky, C. Giunti and W. Grimus, Phenomenology of neutrino oscillations, Prog. Part. Nucl. Phys. 43 (1999) 1 arXiv:hep-ph/9812360.

[65] S. M. Bilenky and S. T. Petcov, Massive neutrinos and neutrino oscillations, Rev. Mod. Phys. 59 (1987) 671 [Erratum-ibid. 61 (1989) 169].

[66] S. M. Bilenky and B. Pontecorvo, Lepton mixing and neutrino oscillations, Phys. Rep. 41 (1978) 225.

[67] M. Binger and C. R. Ji, Quantum field theory of meson mixing, Phys. Rev. D 60 (1999) 056005 arXiv:hep$\mathrm{ph} / 9901407$.

[68] M. Blasone, A. Capolupo, O. Romei and G. Vitiello, Quantum field theory of boson mixing, Phys. Rev. D 63 (2001) 125015 arXiv:hep-ph/0102048.

[69] M. Blasone, A. Capolupo and G. Vitiello, Comment on 'Remarks on flavor-neutrino propagators and oscillation formulae', arXiv:hep-ph/0107183.

[70] M. Blasone, A. Capolupo and G. Vitiello, Quantum field theory of three flavor neutrino mixing and oscillations with CP violation, Phys. Rev. D 66 (2002) 025033 arXiv:hep-th/0204184.

[71] M. Blasone, P. A. Henning and G. Vitiello, The exact formula for neutrino oscillations, Phys. Lett. B 451 (1999) 140 arXiv:hep-th/9803157. 
[72] M. Blasone, P. Jizba and G. Vitiello, Currents and charges for mixed fields, Phys. Lett. B 517 (2001) 471 arXiv:hep-th/0103087.

[73] M. Blasone and G. Vitiello, Quantum field theory of fermion mixing, Ann. Phys. (N.Y.) 244 (1995) 283 [Erratum-ibid. 249 (1996) 363] arXiv:hep-ph/9501263.

[74] M. Blasone and G. Vitiello, Remarks on the neutrino oscillation formula, Phys. Rev. D 60 (1999) 111302 arXiv:hep-ph/9907382.

[75] F. Boehm and P. Vogel, Physics of Massive Neutrinos (Cambridge University Press, Cambridge, 1992) chap. 4.

[76] A. Bramon and G. Garbarino, Novel Bell's inequalities for entangled $K^{0} \bar{K}^{0}$ pairs, Phys. Rev. Lett. 88 (2002) 040403 arXiv:quant-ph/0108047.

[77] G. C. Branco, L. Lavoura and J. P. Silva, CP violation (Clarendon Press, Oxford, 1999).

[78] L. S. Brown, Quantum Field Theory (Cambridge University Press, Cambridge, 1992).

[79] C. Buchanan, R. Cousins, C. Dib, R. D. Peccei and J. Quackenbush, Testing CP and CPT violation in the neutral kaon system at a $\phi$ factory, Phys. Rev. D 45 (1992) 4088.

[80] H. Burkhardt, J. Lowe, G. J. Stephenson and T. Goldman, Oscillations of recoil particles against mixed states, Phys. Rev. D 59 (1999) 054018 [arXiv:hep-ph/9803365].

[81] F. W. Byron and R. W. Fuller, Mathematics of Classical and Quantum Physics (Dover, New York, 1992) p. 170.

[82] A. B. Carter and A. I. Sanda, CP violation in cascade decays of B mesons, Phys. Rev. Lett. 45 (1980) 952.

[83] A. B. Carter and A. I. Sanda, CP violation in B meson decays, Phys. Rev. D 23 (1981) 1567.

[84] J. E. Campagne, Neutrino oscillations from pion decay in flight, Phys. Lett. B 400 (1997) 135.

[85] C. Y. Cardall, Coherence of neutrino flavor mixing in quantum field theory, Phys. Rev. D 61 (2000) 073006 arXiv:hep-ph/9909332.

[86] C. Y. Cardall, Theory of neutrino flavor mixing, arXiv:hep-ph/0107004.

[87] C. Y. Cardall and D. J. Chung, The MSW effect in quantum field theory, Phys. Rev. D 60 (1999) 073012 arXiv:hep-ph/9904291.

[88] C. B. Chiu and E. C. Sudarshan, Decay and evolution of the neutral kaon, Phys. Rev. D 42 (1990) 3712.

[89] S. Choubey and S. Goswami, Is neutrino decay really ruled out as a solution to the atmospheric neutrino problem from Super-Kamiokande data?, Astropart. Phys. 14 (2000) 67 arXiv:hep-ph/9904257.

[90] S. Choubey, S. Goswami and D. Majumdar, Status of the neutrino decay solution to the solar neutrino problem, Phys. Lett. B 484 (2000) 73 arXiv:hep-ph/0004193].

[91] J. H. Christenson, J. W. Cronin, V. L. Fitch and R. Turlay, Evidence for the $2 \pi$ decay of the $K_{2}^{0}$ meson, Phys. Rev. Lett. 13 (1964) 138.

[92] E. D. Church, K. Eitel, G. B. Mills and M. Steidl, Statistical analysis of different $\bar{\nu}_{\mu} \rightarrow \bar{\nu}_{e}$ searches, Phys. Rev. D 66 (2002) 013001 [arXiv:hep-ex/0203023.

[93] B. T. Cleveland et al., Measurement of the solar electron neutrino flux with the Homestake Chlorine Detector, Astrophys. J. 496 (1998) 505.

[94] G. Comsa, Comment on 'Direct Measurement of the longitudinal coherence length of a thermal neutron beam', Phys. Rev. Lett. 51 (1983) 1105; H. Kaiser, S. A. Werner and E. A. George, ibid. 1106; see also Refs. 174, 56, 147.

[95] S. Couvidat, S. Turck-Chieze and A. G. Kosovichev, New solar seismic models and the neutrino puzzle, arXiv:astro-ph/0203107.

[96] M. Czakon, J. Gluza and M. Zrałek, Nonunitary neutrino mixing matrix and CP violating neutrino oscillations, Acta Phys. Polon. B 32 (2001) 3735 arXiv:hep-ph/0109245.

[97] R. Davis, A review of the Homestake solar neutrino experiment, Prog. Part. Nucl. Phys. 32 (1994) 13.

[98] R. J. Davis, D. S. Harmer and K. C. Hoffman, Search for neutrinos from the Sun, Phys. Rev. Lett. 20 (1968) 1205.

[99] H. Davoudiasl, P. Langacker and M. Perelstein, Constraints on large extra dimensions from neutrino oscillation experiments, Phys. Rev. D 65 (2002) 105015 arXiv:hep-ph/0201128, and references therein.

[100] T. B. Day, Demonstration of quantum mechanics in the large, Phys. Rev. 121 (1961) 1204. 
[101] S. De Leo, G. Ducati and P. Rotelli, Comments upon the mass oscillation formulas, Mod. Phys. Lett. A 15 (2000) 2057 arXiv:hep-ph/9906460.

[102] S. De Leo, C. C. Nishi and P. P. Rotelli, Quantum oscillation phenomena, arXiv:hep-ph/0208086.

[103] K. Dick, M. Freund, M. Lindner and A. Romanino, CP-violation in neutrino oscillations, Nucl. Phys. B 562 (1999) 29 arXiv:hep-ph/9903308.

[104] R. H. Dicke and J. P. Wittke, Introduction to Quantum Mechanics (Addison-Wesley, Reading, 1960 ) p. 336.

[105] A. D. Dolgov, Neutrino oscillations and cosmology, arXiv:hep-ph/0004032.

[106] A. D. Dolgov, Neutrinos in cosmology, arXiv:hep-ph/0202122.

[107] A. D. Dolgov, A. Y. Morozov, L. B. Okun and M. G. Shchepkin, Do muons oscillate?, Nucl. Phys. B 502 (1997) 3 arXiv:hep-ph/9703241.

[108] I. Dunietz, J. Hauser and J. L. Rosner, An experiment addressing CP and CPT violation in the $K^{0} \bar{K}^{0}$ system, Phys. Rev. D 35 (1987) 2166.

[109] G. R. Dvali and A. Y. Smirnov, Probing large extra dimensions with neutrinos, Nucl. Phys. B 563 (1999) 63 arXiv:hep-ph/9904211.

[110] C. P. Enz and R. R. Lewis, On the phenomenological description of CP violation for K-Mesons and its consequences, Helv. Phys. Acta 38 (1965) 860.

[111] A. Erdelyi, Asymptotic Expansions (Dover, New York, 1956).

[112] J. H. Field, The description of neutrino and muon oscillations by interfering amplitudes of classical spacetime paths, arXiv:hep-ph/0110064.

[113] P. Fisher, B. Kayser and K. S. McFarland, Neutrino mass and oscillation, Ann. Rev. Nucl. Part. Sci. 49 (1999) 481 arXiv:hep-ph/9906244.

[114] V. L. Fitch, P. A. Piroué and R. B. Perkins, Mass difference of neutral K-meson, Nuovo Cim. 22 (1961) 1160 .

[115] R. Fleischer, CP violation and the role of electroweak penguins in non-leptonic B decays, Int. J. Mod. Phys. A 12 (1997) 2459 arXiv:hep-ph/9612446.

[116] R. Foadi and F. Selleri, Quantum mechanics versus local realism and a recent EPR experiment on $K^{0} \bar{K}^{0}$ pairs, Phys. Lett. B 461 (1999) 123.

[117] G. L. Fogli, E. Lisi, A. Marrone and G. Scioscia, Super-Kamiokande data and atmospheric neutrino decay, Phys. Rev. D 59 (1999) 117303 arXiv:hep-ph/9902267.

[118] N. Fornengo, M. Maltoni, R. T. Bayo and J. W. Valle, Probing neutrino non-standard interactions with atmospheric neutrino data, Phys. Rev. D 65 (2002) 013010 arXiv:hep-ph/0108043, and references therein.

[119] J. A. Frieman, H. E. Haber and K. Freese, Neutrino mixing, decays and supernova 1987 A, Phys. Lett. B 200 (1988) 115.

[120] J. R. Fry, CP violation and the Standard Model, Rep. Prog. Phys. 63 (2000) 117.

[121] Y. Fukuda et al. [Kamiokande Collaboration], Atmospheric $\nu_{\mu} / \nu_{e}$ ratio in the multi-GeV range, Phys. Lett. B 335 (1994) 237.

[122] Y. Fukuda et al. [Kamiokande Collaboration], Solar neutrino data covering solar cycle 22, Phys. Rev. Lett. 77 (1996) 1683.

[123] Y. Fukuda et al. [Super-Kamiokande Collaboration], Measurements of the solar neutrino flux from SuperKamiokande's first 300 days, Phys. Rev. Lett. 81 (1998) 1158 [Erratum-ibid. 81 (1998) 4279] [arXiv:hepex/9805021.

[124] Y. Fukuda et al. [Super-Kamiokande Collaboration], Evidence for oscillation of atmospheric neutrinos, Phys. Rev. Lett. 81 (1998) 1562 arXiv:hep-ex/9807003.

[125] S. Fukuda et al. [Super-Kamiokande Collaboration], Tau neutrinos favored over sterile neutrinos in atmospheric muon neutrino oscillations, Phys. Rev. Lett. 85 (2000) 3999 arXiv:hep-ex/0009001.

[126] S. Fukuda et al. [Super-Kamiokande Collaboration], Solar ${ }^{8} B$ and hep neutrino measurements from 1258 days of Super-Kamiokande data, Phys. Rev. Lett. 86 (2001) 5651 arXiv:hep-ex/0103032.

[127] S. Fukuda et al. [Super-Kamiokande Collaboration], Determination of solar neutrino oscillation parameters using 1496 days of Super-Kamiokande-I data, Phys. Lett. B 539 (2002) 179 arXiv:hep-ex/0205075. 
[128] M. Fukugita and T. Yanagida, Physics of neutrinos, in Physics and Astrophysics of Neutrinos, eds M. Fukugita and A. Suzuki (Springer-Verlag, New York, 1994), p. 92.

[129] K. Fujii, C. Habe and T. Yabuki, Note on the field theory of neutrino mixing, Phys. Rev. D 59 (1999) 113003 [Erratum-ibid. D 60 (1999) 099903] arXiv:hep-ph/9807266].

[130] K. Fujii, C. Habe and T. Yabuki, Remarks on flavor-neutrino propagators and oscillation formulae, Phys. Rev. D 64 (2001) 013011 [arXiv:hep-ph/0102001].

[131] D. Gabor, Theory of electron interference experiments, Rev. Mod. Phys. 28 (1956) 260.

[132] A. M. Gago, M. M. Guzzo, P. C. de Holanda, H. Nunokawa, O. L. Peres, V. Pleitez and R. Zukanovich Funchal, Global analysis of the post-SNO solar neutrino data for standard and non-standard oscillation mechanisms, Phys. Rev. D 65 (2002) 073012 arXiv:hep-ph/0112060.

[133] A. Galindo and P. Pascual, Quantum Mechanics (Springer-Verlag, Berlin, 1990) p. 292.

[134] M. Gell-Mann and A. Pais, Behavior of neutral particles under charge conjugation, Phys. Rev. 97 (1955) 1387.

[135] M. Genovese, C. Novero and E. Predazzi, Can experimental tests of Bell inequalities performed with pseudoscalar mesons be definitive?, Phys. Lett. B 513 (2001) 401 arXiv:hep-ph/0103298.

[136] C. Giunti, Energy and momentum of oscillating neutrinos, Mod. Phys. Lett. A 16 (2001) 2363 arXiv:hepph/0104148.

[137] C. Giunti, The phase of neutrino oscillations, arXiv:hep-ph/0202063.

[138] C. Giunti, Neutrino wave packets in quantum field theory, arXiv:hep-ph/0205014.

[139] C. Giunti and C. W. Kim, Coherence of neutrino oscillations in the wave packet approach, Phys. Rev. D 58 (1998) 017301 arXiv:hep-ph/9711363.

[140] C. Giunti and C. W. Kim, Quantum mechanics of neutrino oscillations, Found. Phys. Lett. 14 (2001) 213 arXiv:hep-ph/0011074.

[141] C. Giunti, C. W. Kim and U. W. Lee, When do neutrinos really oscillate?: Quantum mechanics of neutrino oscillations, Phys. Rev. D 44 (1991) 3635.

[142] C. Giunti, C. W. Kim and U. W. Lee, Comments on the weak states of neutrinos, Phys. Rev. D 45 (1992) 2414.

[143] C. Giunti, C. W. Kim and U. W. Lee, Coherence of neutrino oscillations in vacuum and matter in the wave packet treatment, Phys. Lett. B 274 (1992) 87.

[144] C. Giunti, C. W. Kim and U. W. Lee, When do neutrinos cease to oscillate?, Phys. Lett. B 421 (1998) 237 arXiv:hep-ph/9709494.

[145] C. Giunti, C. W. Kim, J. A. Lee and U. W. Lee, Treatment of neutrino oscillations without resort to weak eigenstates, Phys. Rev. D 48 (1993) 4310 arXiv:hep-ph/9305276.

[146] T. Goldman, Source dependence of neutrino oscillations, arXiv:hep-ph/9604357.

[147] R. Golub and S. K. Lamoreaux, Elucidation of the neutron coherence length and a matter-wave sideband interferometer, Phys. Lett. A 162 (1992) 122.

[148] R. H. Good et al., Regeneration of neutral K mesons and their mass difference, Phys. Rev. 124 (1961) 1223.

[149] V. N. Gribov and B. Pontecorvo, Neutrino astronomy and lepton charge, Phys. Lett. 28B (1969) 493.

[150] W. Grimus and P. Stockinger, Real oscillations of virtual neutrinos, Phys. Rev. D 54 (1996) 3414 arXiv:hep$\mathrm{ph} / 9603430$.

[151] W. Grimus, P. Stockinger and S. Mohanty, The field-theoretical approach to coherence in neutrino oscillations, Phys. Rev. D 59 (1999) 013011 arXiv:hep-ph/9807442].

[152] W. Grimus, S. Mohanty and P. Stockinger, Neutrino oscillations and the effect of the finite lifetime of the neutrino source, Phys. Rev. D 61 (2000) 033001 arXiv:hep-ph/9904285.

[153] M. Gronau and J. L. Rosner, Tests for coherence in neutral B meson decays, Phys. Rev. D 63 (2001) 054006 [Erratum-ibid. D 64 (2001) 099902] arXiv:hep-ph/0010238].

[154] D. E. Groom, Understanding two-flavor oscillation parameters and limits, in Ref. [156].

[155] Y. Grossman and H. J. Lipkin, Flavor oscillations from a spatially localized source: a simple general treatment, Phys. Rev. D 55 (1997) 2760 arXiv:hep-ph/9607201. 
[156] K. Hagiwara et al. [Particle Data Group Collaboration], Review of Particle Physics, Phys. Rev. D 66 (2002) 010001.

[157] W. Hampel et al. [GALLEX Collaboration], GALLEX solar neutrino observations: Results for GALLEX IV, Phys. Lett. B 447 (1999) 127.

[158] K. C. Hannabuss and D. C. Latimer, The quantum field theory of fermion mixing, J. Phys. A 33 (2000) 1369.

[159] M. Hayakawa and A. I. Sanda, Searching for T, CP, CPT and $\Delta S=\Delta Q$ rule violations in the neutral K meson system a guide, Phys. Rev. D 48 (1993) 1150 arXiv:hep-ph/9302206.

[160] K. S. Hirata et al. [Kamiokande-II Collaboration], Experimental study of the atmospheric neutrino flux, Phys. Lett. B 205 (1988) 416.

[161] K. S. Hirata et al. [Kamiokande-II Collaboration], Observation Of ${ }^{8} B$ Solar neutrinos in the Kamiokande-II detector, Phys. Rev. Lett. 63 (1989) 16.

[162] D. R. Inglis, Completeness of quantum mechanics and charge-conjugation correlations of theta particles, Rev. Mod. Phys. 33 (1961) 1.

[163] A. Ioannisian and A. Pilaftsis, Neutrino oscillations in space within a solvable model, Phys. Rev. D 59 (1999) 053003 arXiv:hep-ph/9809503.

[164] T. Ishii [K2K Collaboration], New results from K2K, arXiv:hep-ex/0206033.

[165] C. Itzykson and J.-B. Zuber, Quantum Field Theory (McGraw-Hill, New York, 1980).

[166] R. Jacob and R. G. Sachs, Mass and lifetime of unstable particles, Phys. Rev. 121 (1961) 350.

[167] C. R. Ji and Y. Mishchenko, Nonperturbative vacuum effect in the quantum field theory of meson mixing, Phys. Rev. D 64 (2001) 076004 arXiv:hep-ph/0105094.

[168] C. R. Ji and Y. Mishchenko, The general theory of quantum field mixing, Phys. Rev. D 65 (2002) 096015 arXiv:hep-ph/0201188.

[169] A. S. Joshipura, E. Masso and S. Mohanty, Constraints on decay plus oscillation solutions of the solar neutrino problem, arXiv:hep-ph/0203181.

[170] C. K. Jung, C. McGrew, T. Kajita and T. Mann, Oscillations of atmospheric neutrinos, Ann. Rev. Nucl. Part. Sci. 51 (2001) 451.

[171] P. K. Kabir, The CP Puzzle (Academic Press, New York, 1968), and references therein.

[172] P. K. Kabir, in Ref. [171] p. 99.

[173] F. A. Kaempffer, Concepts in Quantum Mechanics (Academic Press, New York, 1965) p. 341.

[174] H. Kaiser, S. A. Werner and E. A. George, Direct measurement of the longitudinal coherence length of a thermal neutron beam, Phys. Rev. Lett. 50 (1983) 560.

[175] T. Kajita and Y. Totsuka, Observation of atmospheric neutrinos, Rev. Mod. Phys. 73 (2001) 85.

[176] B. Kayser, On the quantum mechanics of neutrino oscillation, Phys. Rev. D 24 (1981) 110.

[177] B. Kayser, CP violation, mixing, and quantum mechanics, arXiv:hep-ph/9702327.

[178] B. Kayser, The frequency of neutral meson and neutrino oscillation, SLAC-PUB-7123 (March 1997) http://www.slac.stanford.edu/pubs/slacpubs/7000/slac-pub-7123.htm.

[179] B. Kayser, Cascade mixing and the CP-violating angle beta, arXiv:hep-ph/9709382.

[180] B. Kayser, Neutrino physics as explored by flavor change, in Ref. [156].

[181] B. Kayser and L. Stodolsky, EPR experiments without 'collapse of the wave function', Phys. Lett. B 359 (1995) 343.

[182] K. Kiers, S. Nussinov and N. Weiss, Coherence effects in neutrino oscillations, Phys. Rev. D 53 (1996) 537 arXiv:hep-ph/9506271.

[183] K. Kiers and N. Weiss, Neutrino oscillations in a model with a source and detector, Phys. Rev. D 57 (1998) 3091 arXiv:hep-ph/9710289.

[184] C. W. Kim and A. Pevsner, Neutrinos in Physics and Astrophysics (Harwood Academic Publishers, Chur, 1993) chap. 12.

[185] C. W. Kim and A. Pevsner, in Ref. 184] chap. 7 and 17. 
[186] A. G. Klein, G. I. Opat and W. A. Hamilton, Longitudinal coherence in neutron interferometry, Phys. Rev. Lett. 50 (1983) 563.

[187] I. Yu. Kobzarev, B. V. Martemyanov, L. B. Okun and M. G. Shchepkin, Sum rules for neutrino oscillations, Sov. J. Nucl. Phys. 35 (1982) 708 [Yad. Fiz. 35 (1982) 1210].

[188] L. Krauss and F. Wilczek, Solar neutrino oscillations, Phys. Rev. Lett. 55 (1985) 122.

[189] T. K. Kuo and J. Pantaleone, Neutrino oscillations in matter, Rev. Mod. Phys. 61 (1989) 937.

[190] K. Landé, E. T. Booth, J. Impeduglia, L. M. Lederman and W. Chinowsky, Observation of long-lived neutral V particles, Phys. Rev. 103 (1956) 1901(L).

[191] T. D. Lee, R. Oehme and C. N. Yang, Remarks on possible noninvariance under time reversal and charge conjugation, Phys. Rev. 106 (1957) 340.

[192] M. Lindner, T. Ohlsson and W. Winter, A combined treatment of neutrino decay and neutrino oscillations, Nucl. Phys. B 607 (2001) 326 [arXiv:hep-ph/0103170].

[193] M. Lindner, T. Ohlsson and W. Winter, Decays of supernova neutrinos, Nucl. Phys. B 622 (2002) 429 arXiv:astro-ph/0105309.

[194] B. Linet and P. Teyssandier, Quantum phase shift and neutrino oscillations in a stationary, weak gravitational field, arXiv:gr-qc/0206056.

[195] P. Lipari and M. Lusignoli, On exotic solutions of the atmospheric neutrino problem, Phys. Rev. D 60 (1999) 013003 arXiv:hep-ph/9901350.

[196] H. J. Lipkin, CP violation and coherent decays of kaon pairs, Phys. Rev. 176 (1968) 1715.

[197] H. J. Lipkin, Theories of nonexperiments in coherent decays of neutral mesons, Phys. Lett. B 348 (1995) 604 arXiv:hep-ph/9501269].

[198] H. J. Lipkin, Quantum mechanics of neutrino oscillations: Hand waving for pedestrians, arXiv:hep$\mathrm{ph} / 9901399$.

[199] E. Lisi, A. Marrone and D. Montanino, Probing possible decoherence effects in atmospheric neutrino oscillations, Phys. Rev. Lett. 85 (2000) 1166 arXiv:hep-ph/0002053.

[200] A. Loeb, Collisional incoherence in neutrino line emission, Phys. Rev. D 39 (1989) 1009.

[201] J. Lowe, B. Bassalleck, H. Burkhardt, A. Rusek, G. J. Stephenson and T. Goldman, No $\Lambda$ oscillations, Phys. Lett. B 384 (1996) 288 arXiv:hep-ph/9605234.

[202] E. Ma, G. Rajasekaran and I. Stancu, Hierarchical four-neutrino oscillations with a decay option, Phys. Rev. D 61 (2000) 071302 [arXiv:hep-ph/9908489.

[203] Z. Maki, M. Nakagawa and S. Sakata, Remarks on the unified model of elementary particles, Prog. Theor. Phys. 28 (1962) 870.

[204] L. Malyshkin and R. Kulsrud, Effect of Coulomb collisions on time variations of the solar neutrino flux, Mon. Not. Roy. Astron. Soc. 316 (2000) 249 arXiv:astro-ph/9901015.

[205] P. D. Mannheim, Derivation of the formalism for neutrino matter oscillations from the neutrino relativistic field equations, Phys. Rev. D 37 (1988) 1935.

[206] S. P. Mikheyev and A. Y. Smirnov, Resonance enhancement of oscillations in matter and solar neutrino spectroscopy, Sov. J. Nucl. Phys. 42 (1985) 913 [Yad. Fiz. 42 (1985) 1441].

[207] S. P. Mikheyev and A. Y. Smirnov, Resonant amplification of $\nu$ oscillations in matter and solar-neutrino spectroscopy, Nuovo Cim. C 9 (1986) 17.

[208] R. N. Mohapatra and P. B. Pal, Massive Neutrinos in Physics and Astrophysics (World Scientific, Singapore, 1991).

[209] O. Nachtmann, Elementary Particle Physics (Springer-Verlag, Berlin, 1990) Appendix I.

[210] O. Nachtmann, in Ref. [209] chap. 26.

[211] K. Nakamura, Solar neutrinos, in Ref. 156.

[212] M. Nauenberg, Correlated wave packet treatment of neutrino and neutral meson oscillations, Phys. Lett. B 447 (1999) 23 arXiv:hep-ph/9812441.

[213] Y. Nir and H. R. Quinn, Theory of CP violation in B decays, in B decays (rev. 2nd ed.), ed. S. Stone (World Scientific, Singapore, 1994) p. 520. 
[214] D. Nötzold and G. Raffelt, Neutrino dispersion at finite temperature and density, Nucl. Phys. B 307 (1988) 924.

[215] M. Nowakowski, Beyond the Weisskopf-Wigner approximation in the $K^{0} \bar{K}^{0}$ system, Int. J. Mod. Phys. A 14 (1999) 589.

[216] S. Nussinov, Solar neutrinos and neutrino mixing, Phys. Lett. 63B (1976) 201.

[217] L. B. Okun, On neutrino oscillations, Surveys High Energy Physics 15 (2000) 75 [available at http://www.to.infn.it/ ${ }^{\sim}$ giunti/neutrino/qmno/].

[218] L. B. Okun and I. S. Tsukerman, Comment on equal velocity assumption for neutrino oscillations, Mod. Phys. Lett. A 15 (2000) 1481 arXiv:hep-ph/0007262].

[219] A. Pais and O. Piccioni, Note on the decay and absorption of the $\theta^{0}$, Phys. Rev. 100 (1955) 1487.

[220] S. Pakvasa, Neutrino anomalies without oscillations, Pramana 54 (2000) 65 [arXiv:hep-ph/9910246.

[221] S. Pakvasa and J. Pantaleone, Direct probes of neutrino properties using solar-neutrino lines, Phys. Rev. Lett. 65 (1990) 2479.

[222] S. Pakvasa and K. Tennakone, Neutrinos of nonzero rest mass, Phys. Rev. Lett. 28 (1972) 1415.

[223] P. B. Pal and T. N. Pham, Field-theoretic derivation of Wolfenstein's matter-oscillation formula, Phys. Rev. D 40 (1989) 259.

[224] S. J. Parke, Nonadiabatic level crossing in resonant neutrino oscillations, Phys. Rev. Lett. 57 (1986) 1275.

[225] J. T. Peltoniemi and V. Sipiläinen, Neutrino propagation in matter using the wave packet approach, JHEP 0006 (2000) 011 arXiv:hep-ph/0004162.

[226] M. E. Peskin and D. V. Schroeder, An Introduction to Quantum Field Theory (Addison-Wesley, Reading, 1995) p. 102.

[227] S. T. Petcov and J. Rich, The effects of averaging on the matter-enhanced oscillations of solar neutrinos, Phys. Lett. B 224 (1989) 426.

[228] A. Pompili and F. Selleri, On a possible EPR experiment with $B_{d}^{0} \bar{B}_{d}^{0}$ pairs, Eur. Phys. J. C 14 (2000) 469 arXiv:hep-ph/9906347.

[229] B. Pontecorvo, Mesonium and antimesonium, Sov. Phys. JETP 6 (1957) 429 [Zh. Eksp. Teor. Fiz. 33 (1957) 549].

[230] B. Pontecorvo, Inverse beta processes and nonconservation of lepton charge, Sov. Phys. JETP 7 (1958) 172 [Zh. Eksp. Teor. Fiz. 34 (1957) 247].

[231] B. Pontecorvo, Neutrino experiments and the problem of conservation of leptonic charge, Sov. Phys. JETP 26 (1968) 984 [Zh. Eksp. Teor. Fiz. 53 (1967) 1717].

[232] M. Prakash, J. M. Lattimer, R. F. Sawyer and R. R. Volkas, Neutrino propagation in dense astrophysical systems, Ann. Rev. Nucl. Part. Sci. 51 (2001) 295 arXiv:astro-ph/0103095.

[233] H. R. Quinn, T. Schietinger, J. P. Silva and A. E. Snyder, Using kaon regeneration to probe the quark mixing parameter $\cos (2 \beta)$ in $B \rightarrow \psi K$ decays, Phys. Rev. Lett. 85 (2000) 5284 arXiv:hep-ph/0008021].

[234] G.G. Raffelt, Stars as Laboratories for Fundamental Physics (University of Chicago Press, Chicago, 1996).

[235] A. Raychaudhuri and A. Sil, Violation of the equivalence principle in the light of the SNO and SK solar neutrino results, Phys. Rev. D 65 (2002) 073035 arXiv:hep-ph/0107022, and references therein.

[236] P. Reinartz and L. Stodolsky, Neutrino masses and mixings in supernova bursts, Z. Phys. C 27 (1985) 507.

[237] J. Rich, The quantum mechanics of neutrino oscillations, Phys. Rev. D 48 (1993) 4318.

[238] R. G. Sachs, Interference phenomena of neutral K mesons, Ann. Phys. (N.Y.) 22 (1963) 239.

[239] E. Sassaroli, Neutrino oscillations: a relativistic example of a two-level system, Am. J. Phys. 67 (1999) 869.

[240] J. Schechter and J. W. Valle, Neutrino masses in SU(2) X U(1) theories, Phys. Rev. D 22 (1980) 2227.

[241] J. Schwinger, Field theory of unstable particles, Ann. Phys. (N.Y) 9 (1960) 169.

[242] Yu. V. Shtanov, Space-time description of neutrino flavour oscillations, Phys. Rev. D 57 (1998) 4418 arXiv:hep-ph/9706378.

[243] J. P. Silva, On the use of the reciprocal basis in neutral meson mixing, Phys. Rev. D 62 (2000) 116008 arXiv:hep-ph/0007075.

[244] Y. N. Srivastava, A. Widom and E. Sassaroli, $\Lambda$ oscillations, Phys. Lett. B 344 (1995) 436. 
[245] Y. Srivastava, A. Widom and E. Sassaroli, Spatial correlations in two neutral kaon decays, Z. Phys. C 66 (1995) 601.

[246] Y. N. Srivastava, A. Widom and E. Sassaroli, Lepton oscillations, arXiv:hep-ph/9509261.

[247] Y. Srivastava, A. Widom and E. Sassaroli, Charged lepton and neutrino oscillations, Eur. Phys. J. C 2 (1998) 769.

[248] L. Stodolsky, The unnecessary wavepacket, Phys. Rev. D 58 (1998) 036006 arXiv:hep-ph/9802387.

[249] D. Sudarsky, E. Fischbach, C. Talmadge, S. H. Aronson and H.-Y. Cheng, Effects of external fields on the neutral kaon system, Ann. Phys. (N.Y) 207 (1991) 103.

[250] Y. Takeuchi, Y. Tazaki, S. Y. Tsai and T. Yamazaki, Wave packet approach to the equal energy/momentum/velocity prescriptions of neutrino oscillations, Mod. Phys. Lett. A 14 (1999) 2329.

[251] Y. Takeuchi, Y. Tazaki, S. Y. Tsai and T. Yamazaki, How do neutrinos propagate? Wave packet treatment of neutrino oscillation, Prog. Theor. Phys. 105 (2001) 471 arXiv:hep-ph/0006334.

[252] I. S. Tsukerman, Do neutrino oscillations allow an extra phenomenological parameter?, JETP Lett. 73 (2001) 380 [Pisma Zh. Eksp. Teor. Fiz. 73 (2001) 424 [Erratum-ibid. 74 (2001) 442]] [arXiv:hep-ph/0103109].

[253] M. J. Veltman, Unitarity and causality in a renormalizable field theory with unstable particles, Physica 29 (1963) 186.

[254] Q. Wang and A. I. Sanda, Neutral kaon system reinvestigated, Phys. Rev. D 55 (1997) 3131.

[255] R. G. Winter, Neutrino oscillation kinematics, Lett. Nuovo Cim. 30 (1981) 101.

[256] L. Wolfenstein, Neutrino oscillations in matter, Phys. Rev. D 17 (1978) 2369.

[257] J. Wudka, Mass dependence of the gravitationally-induced wave-function phase, Phys. Rev. D 64 (2001) 065009 [arXiv:gr-qc/0010077], and references therein.

[258] M. Zrałek, From kaons to neutrinos: quantum mechanics of particle oscillations, Acta Phys. Polon. B 29 (1998) 3925 arXiv:hep-ph/9810543]. 